Doctoral Thesis

\title{
Neural efficiency of a specific cognitive training in working memory in multiple sclerosis patients
}

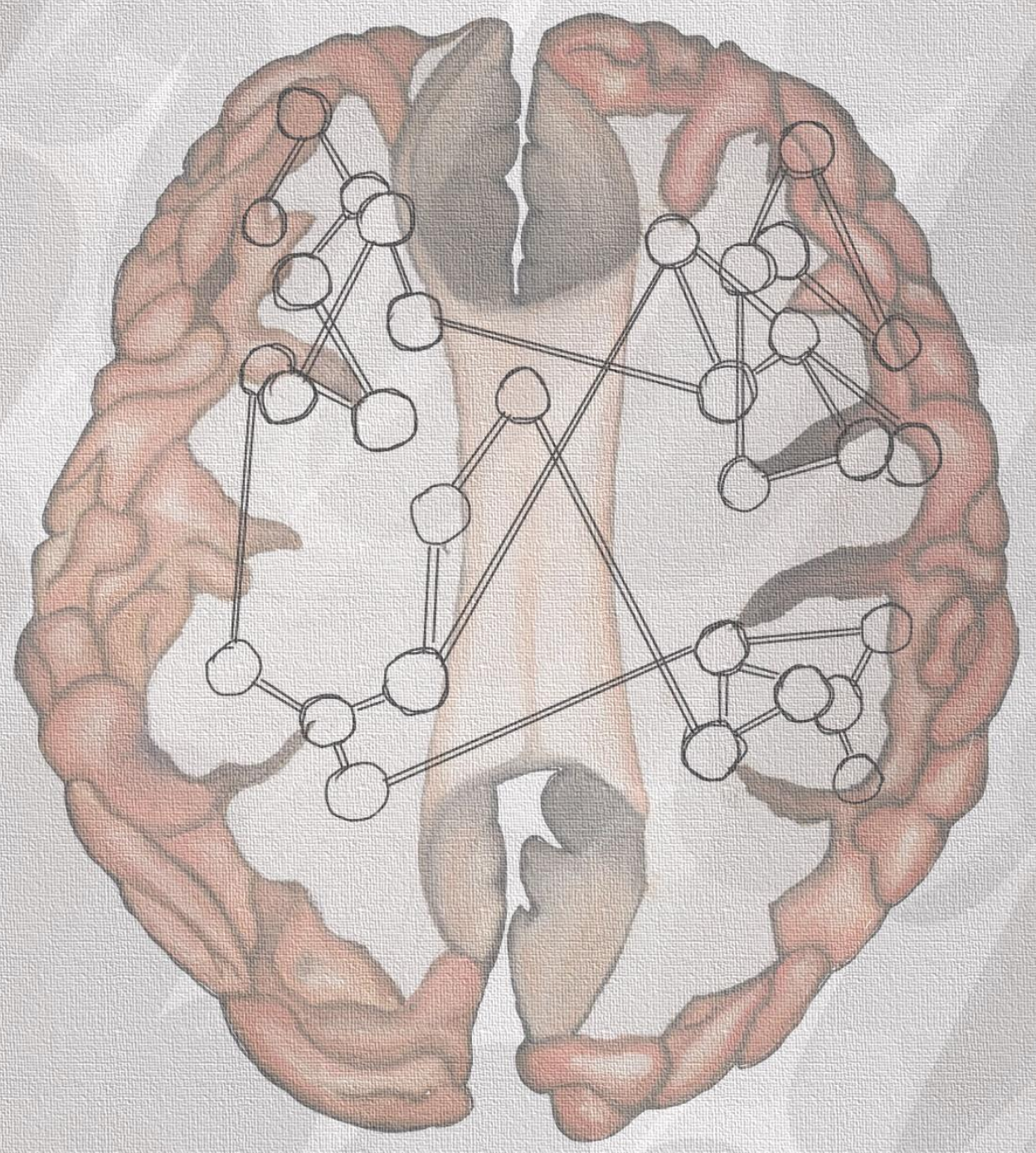

Naiara Aguirre Vidal

Supervisors:

Cristina Forn Frias

César Ávila Rivera

Julio 2021 


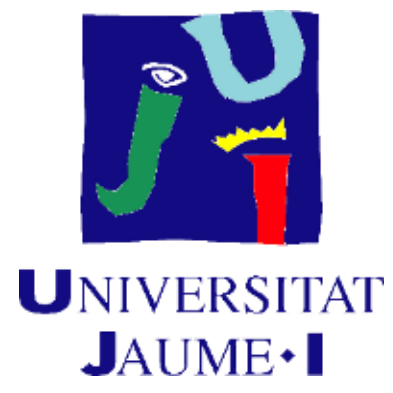

Programa de Doctorado en Psicología

Escuela de Doctorado de la Universidad Jaume I

\section{Neural efficiency of a specific cognitive training in working memory in multiple sclerosis patients}

Memoria presentada por Naiara Aguirre Vidal para optar al grado de doctora por la Universidad Jaume I

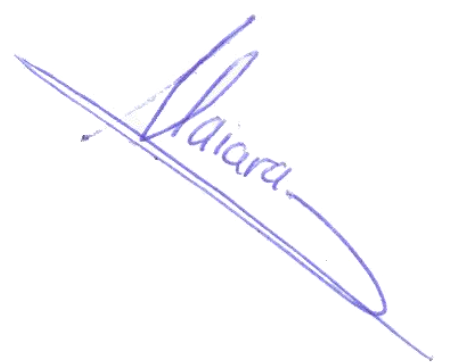

Naiara Aguirre Vidal

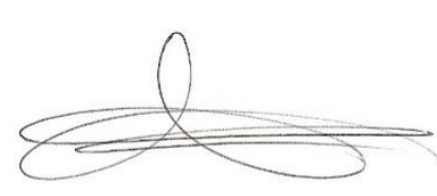

Cristina Forn Frias

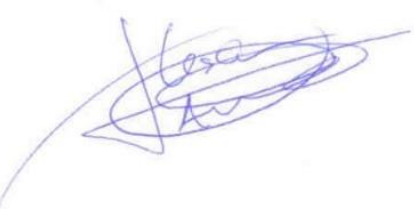

César Ávila Rivera

Castellón de la Plana, julio de 2021 


\section{Financiación recibida}

Esta tesis doctoral ha sido posible gracias a la financiación recibida por parte del Ministerio de Educación, Cultura y Deporte [Ayuda para la formación de profesorado universitario (FPU16/01525)] y por el Ministerio de Ciencia, Innovación y Universidades [Ayuda de movilidad para estancias breves].

Adicionalmente, también se ha contado con la financiación recibida por parte del Ministerio de Economía y Empresa asociada al proyecto de investigación PSI2013-47504-R "Efectos cerebrales a largo plazo tras entrenamiento en funciones cognitivas asociadas a las redes parieto-frontales" 


\section{Tesis por compendio de publicaciones}

- Lesion-Functional Disconnectomics in Multiple Sclerosis

Naiara Aguirre*, Will Orwig, Álvaro-Javier Cruz-Gómez, Anna Miró-Padilla, Elisenda

Bueichekú, César Ávila, Carla Sanchis-Segura, Cristina Forn and Jorge Sepulcre

(Under review)

- Repeated working memory training improves neural efficiency and cognitive performance in multiple sclerosis patients and healthy controls

Naiara Aguirre*, Álvaro Javier Cruz-Gómez, Anna Miró-Padilla, Elisenda Bueichekú, Ricardo Broseta Torres, César Ávila, Carla Sanchis-Segura, Cristina Forn

Revista: Multiple Sclerosis International

doi: 10.1155/2019/2657902

Factor de impacto SJR: 0.53 (2018)

- Enhanced frontoparietal connectivity in multiple sclerosis patients and healthy controls in response to an intensive computerized training focused on working memory

Naiara Aguirre, Álvaro Javier Cruz-Gómez, Sonia Félix Esbrí, Anna Miró-Padilla, Elisenda Bueichekú, Ricardo Broseta-Torres, César Ávila, Carla Sanchis-Segura and Cristina Forn Revista: Multiple Sclerosis and Related Disorders

doi: 10.1016/j.msard.2021.102976

Factor de impacto JCR: 4.339 (2020)

Esta tesis dispone de la aceptación de los coautores de las publicaciones que el doctorando/a presenta como tesis y su renuncia expresa a presentarlas como parte de otra tesis doctoral. 
La elaboración de una tesis doctoral no es un trabajo individual, sino en equipo, que conlleva mucho tiempo y esfuerzo por parte de muchas personas. Por ello, me gustaría agradecer a todos aquellos que me han guiado y apoyado a lo largo de esta tesis.

En primer lugar, me gustaría agradecer a mis directores de tesis, la Dra. Cristina Forn y el Dr. César Ávila por guiarme en cada paso de este trabajo. La gran diferencia en vuestros estilos de enseñanza ha sido una experiencia aún más enriquecedora. Cristina, gracias por tu tenacidad, esfuerzo y generosidad para ayudarme y enseñarme. Muchas gracias por tu paciencia para trabajar con mi personalidad “dispersa”. Sé que no siempre es fácil captar mi atención y conseguir que me centre en un trabajo muy concreto, pero por ello agradezco tu capacidad de dirección y de guía. César, muchas gracias por permitirme ser parte de este equipo, por crearlo y fomentar un ambiente de trabajo colaborativo. Gracias por mostrarme tu apoyo a lo largo de todas las etapas de la tesis, y darme la oportunidad de aprender una gran variedad de conocimientos y habilidades.

También me gustaría incluir aquí a la Dra. Carla Sanchís. Carla, te considero mi directora de tesis, aunque no haya sido posible incluirte de manera oficial. Agradezco que hayas compartido de forma tan generosa y desinteresada todo el conocimiento que tienes, e incluso más. Muchas gracias por todo el tiempo que has empleado en formarme y ayudarme, por el apoyo que me has dado y por tu contagiosa curiosidad por el mundo.

En segundo lugar, me gustaría agradecer a mis compañeros de grupo. En especial, me gustaría agradecer a Javi y a Víctor todo su esfuerzo y paciencia para enseñarme en mis primeras etapas del doctorado. Javi, muchas gracias por todo tu apoyo, dedicación, trabajo y organización. Gracias por todos los momentos compartidos, ¡incluyendo los sushis! Víctor, muchas gracias por tu generosidad para compartir tus aparentemente ilimitados conocimientos metodológicos, y por tu enorme paciencia para explicarme mis dudas tantas veces como fuera necesario. Ambos habéis sido un gran ejemplo para mí. También me gustaría agradecer su disposición y ayuda a Anna y a Eli. Gracias por abrir el camino en la investigación de la memoria de trabajo, y por compartir vuestros conocimientos conmigo. Vuestra dedicación y minuciosidad ha sido de gran ayuda para la continuación con esta línea de trabajo. A mi compañero Jesús, el más reciente doctor del grupo, me gustaría agradecer su amabilidad y su ayuda. Gracias por tu energía contagiosa, y por todos los debates improvisados que directa o indirectamente han aportado calidad a mi trabajo y aprendizaje. A Esteban y Lidón, muchas gracias por vuestro compañerismo y apoyo. Mucho ánimo con la finalización, no muy lejana, de 
vuestras tesis doctorales. He tenido la suerte de poder seguir parte de vuestro trabajo, y ser testigo de vuestro esfuerzo, por lo que estoy convencida de que serán trabajos de gran calidad. A Sonia y Alba, mis más recientes compañeras, mucho ánimo con vuestro inicio en la trayectoria de vuestras tesis. Muchas gracias por vuestro apoyo, y por vuestra curiosidad. Os animo a aprender todo lo posible de esta experiencia, que no sólo aporta al conocimiento académico sino también al personal. Además, Carla y Cristina, agradezco que me hayáis permitido participar en la creación de vuestro nuevo grupo de investigación (ImagoBrain). Estoy convencida de que, con vosotras y el trabajo de las nuevas componentes, Sonia y Alba, este grupo tendrá una gran trayectoria. También me gustaría agradecer al resto componentes del grupo: Marian, Maya, Noe, Lara, Helena y Alfonso, por contribuir a un espacio de trabajo tan agradable y colaborativo.

En tercer lugar, me gustaría agradecer al Dr. Jorge Sepulcre por permitirme disfrutar de la estancia de investigación en un grupo de investigación tan excelente. Agradezco tu disposición para ayudarme, tu amabilidad y tu generosidad. Gracias por todos los conocimientos compartidos conmigo, y por facilitarme la oportunidad de participar en experiencias tan variadas. Gracias también por tus esfuerzos en crear y mantener un grupo de trabajo tan agradable y acogedor. Gracias a todos los integrantes del grupo e investigadores con los que tuve la suerte de compartir mi tiempo de estancia: Ibai, Will, Elena, Chanmie, Víctor, Nicoleta, Pablo, Alfonso... gracias por vuestra amabilidad y por todas las experiencias compartidas. Gracias también a Edward y Brian por hacerme sentir como en casa.

También me gustaría hacer una mención especial a la Asociación de Esclerosis Múltiple de Castellón por su gran disposición para colaborar, y también a todos los participantes que no forman parte de esta asociación. Muchas gracias por hacer el esfuerzo de venir a participar en los estudios.

Por último, me gustaría agradecer a mi familia por todo el apoyo que me han brindado desde siempre. Gracias a mis padres por incentivar mi curiosidad y enseñarme la motivación por el trabajo y el esfuerzo. Gracias Aitana por tu apoyo como hermana, ¡y muchas gracias por la ayuda con el diseño de la portada! Gracias Alberto por tu inquebrantable paciencia y apoyo en todos estos años. Y gracias a todos mis amigos que me han apoyado y animado durante estos años y muchos más.

A todos, ¡muchísimas gracias! 


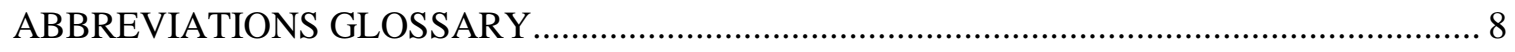

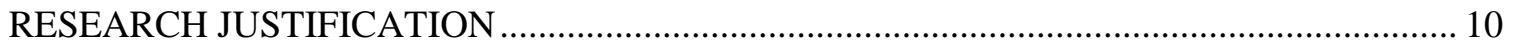

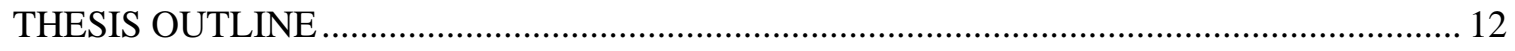

CHAPTER 1 - GENERAL INTRODUCTION .................................................................................... 13

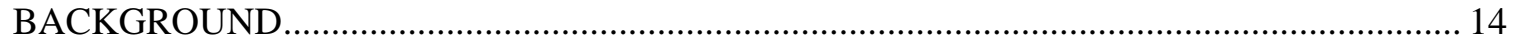

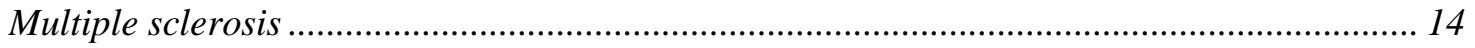

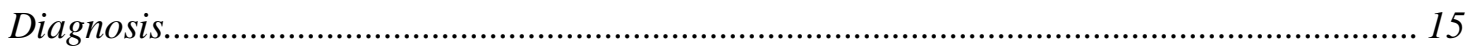

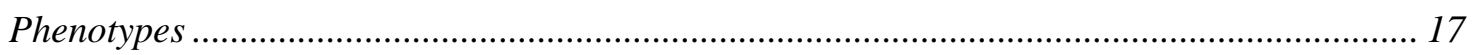

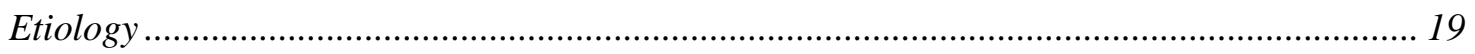

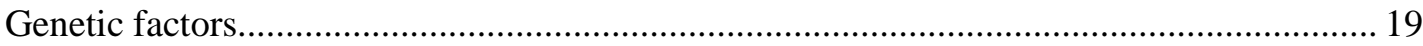

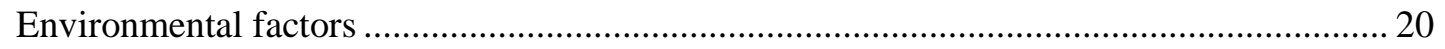

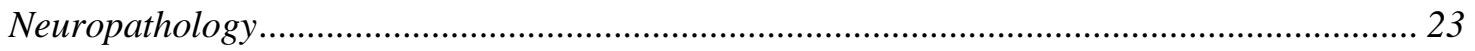

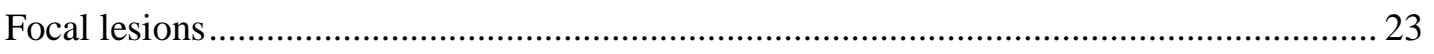

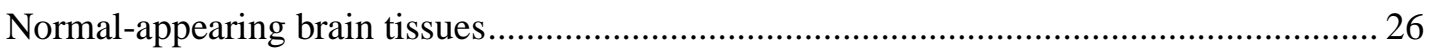

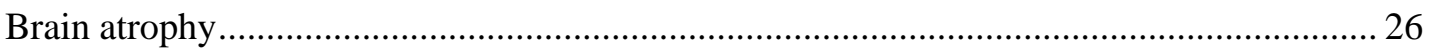

Neuropathology and cognitive decline in multiple sclerosis............................................. 27

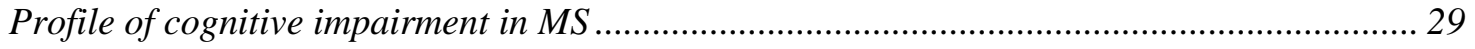

Information processing speed, attention and working memory ......................................... 29

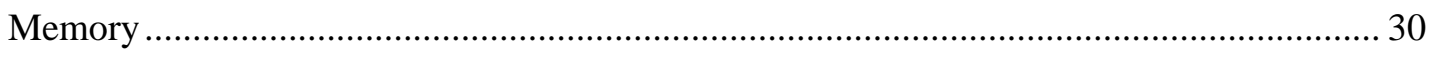

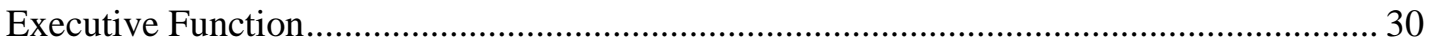

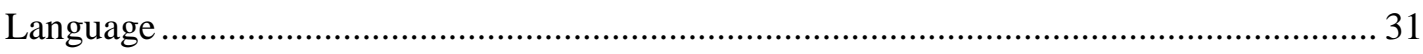

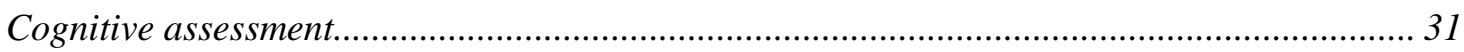

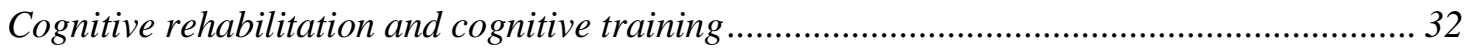




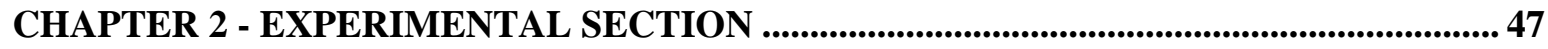

Study I: Lesion-Functional Disconnectomics in Multiple Sclerosis....................................... 48

Study II: Repeated working memory training improves neural efficiency and cognitive performance in multiple sclerosis patients and healthy controls .......................................... 71

Study III: Enhanced frontoparietal connectivity in multiple sclerosis patients and healthy controls in response to an intensive computerized training focused on working memory........ 94

CHAPTER 3 - GENERAL DISCUSSION .......................................................................................... 113

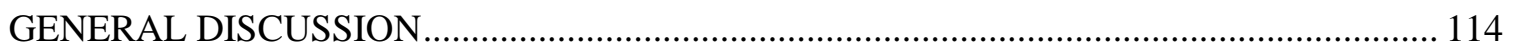

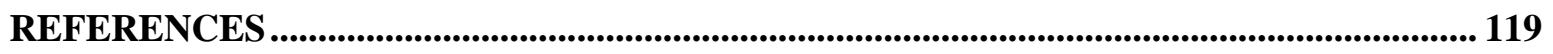




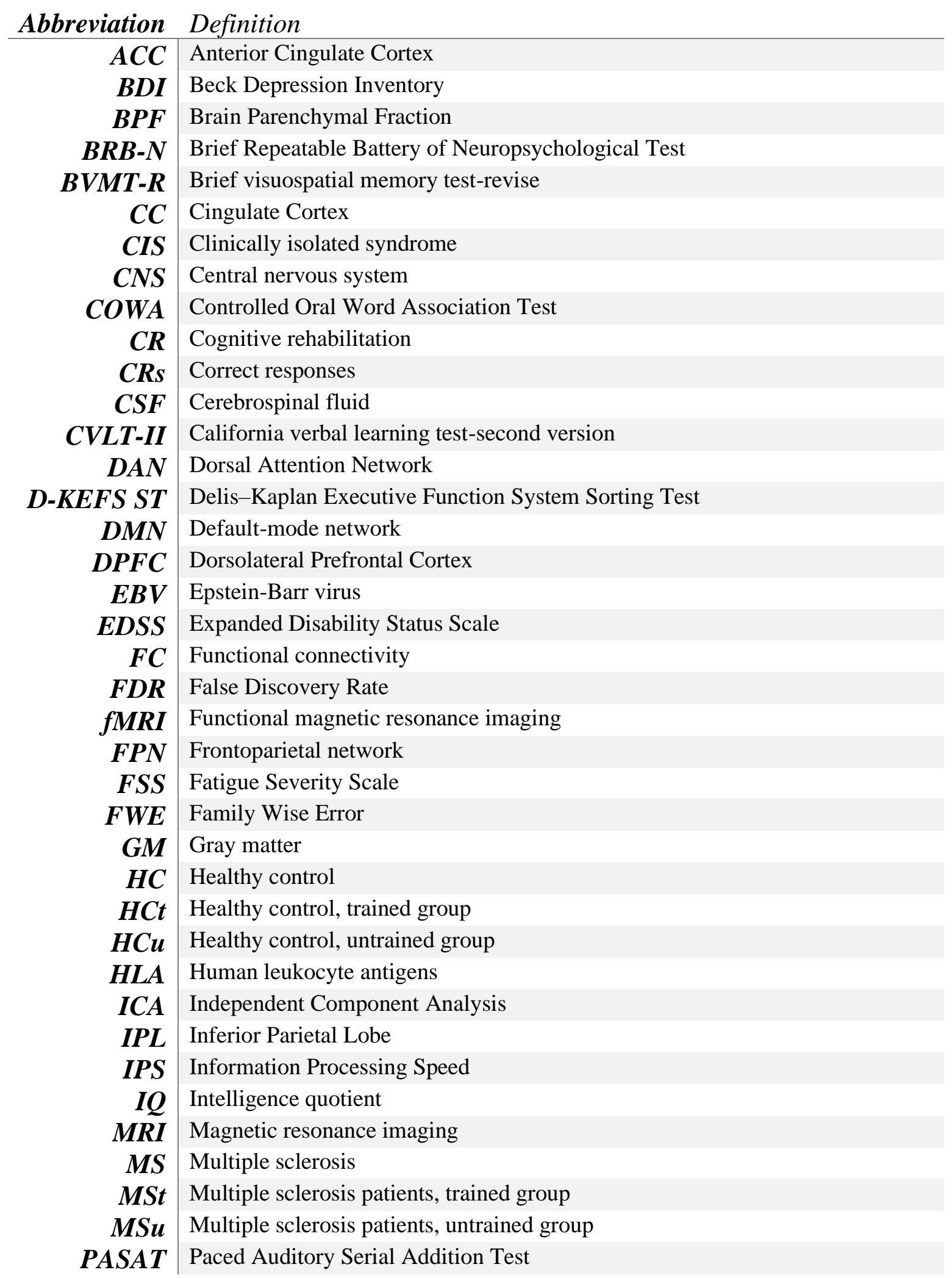




\begin{tabular}{|c|c|}
\hline$P C C$ & Posterior Cingulate Cortex \\
\hline PPMS & Primary-progressive multiple sclerosis \\
\hline PRMS & Progressive-relapsing multiple sclerosis \\
\hline$P V S A T$ & Paced Visual Serial Addition Test \\
\hline$R I S$ & Radiologically isolated syndrome \\
\hline$R R M S$ & Relapsing-remitting multiple sclerosis \\
\hline$R S$ & Resting State \\
\hline$r s F C$ & Resting-state functional connectivity \\
\hline$R T$ & Reaction time \\
\hline$R T s$ & Reaction times \\
\hline$S D M T$ & Symbol Digit Modalities Test \\
\hline$S F C$ & Stepwise Functional Connectivity \\
\hline$S M A$ & Supplementary Motor Area \\
\hline $\mathbf{S M N}$ & Sensory Motor Network \\
\hline SPART & Spatial Recall Test \\
\hline SPMS & Secondary-progressive multiple sclerosis \\
\hline$S R T$ & Selective Reminding Test \\
\hline$T M T$ & Trail Making Test \\
\hline tru & trunk region \\
\hline ulhf & upper limb, head and face region \\
\hline $\boldsymbol{V A N}$ & Ventral Attention Network \\
\hline$V N$ & Visual Network \\
\hline VPFC & Ventral Prefrontal Cortex \\
\hline WAIS-III & Wechsler Adult Intelligence Scale III \\
\hline$W D$ & Weighted Degree \\
\hline$W L G T$ & Word List Generation Test \\
\hline$W M$ & White matter \\
\hline$W M F$ & Working memory function \\
\hline
\end{tabular}




\section{RESEARCH JUSTIFICATION}

Two old friends have arranged to meet up to go hiking in the mountains. They walk, talking and observing the beautiful scenery along a path of slippery stones near a river. Unfortunately, they both slip and twist an ankle. The walk is over, and they have to call another friend who picks them up and takes them to the hospital. Once at the hospital, the doctor evaluates the injury of each of them. Although they just had the same accident, the effect on each of them is not the same. One of them has a slight first-degree sprain, and the doctor puts a support bandage on his ankle and tells him that he can continue walking with care and the pain will go away in a few days. However, his friend has suffered a third-degree sprain, and so the doctor puts him in a cast to immobilize his ankle and tells him he will have to be on crutches for at least two weeks. From then on, he will have to undergo rehabilitation to be able to walk normally again. Surprised, he asks the doctor why there is such a difference between his injury and the one suffered by his friend. The doctor explains that his friend exercises frequently, as a result of which his muscle fibers are stronger and more flexible. Therefore, although they had the same accident, his friend's body was more prepared to counteract the damage.

This example allows us to easily understand the importance of training. We have often experienced or seen similar situations. In fact, we are aware of this when we are climbing the stairs and are out of breath. However, we are less aware of the lack of cognitive exercise, and yet the same mechanism is followed. Indeed, we will rarely find people who are concerned about how "little cognitive training they are doing". Although cognitive health and, therefore, the state of our brain is one of the most important aspects of people's lives, and it directly affects their quality of life. Cognitive training is of great importance for people of any age, healthy or with neurodegenerative disease. Similar to what was explained in the previous example, cognitive exercise makes our brain stronger and more flexible, so that it is better prepared to face any possible damage it may suffer.

In this regard, cognitive training has become a major tool to counteract cognitive impairment in patients with neurodegenerative diseases. In the present thesis, we have focused on multiple sclerosis (MS), which is an unpredictable disease that presents in young adults (around 30 years) and has a great impact on the individual's quality of life, as well as society as a whole. It is characterized by the appearance of spontaneous lesions in the brain that produce cognitive and physical alterations. Cognitive training is a recent concept in the field of MS. This disease has traditionally been considered a physical disease, due to its striking motor and sensory symptomatology. For this reason, a large number of rehabilitation programs have been developed to treat physical disability. However, in the 1980s, more attention began to be paid to the cognitive symptoms of the disease, for the first 
time describing the profile of cognitive impairment characteristic of the disease. A few years later, in the 1990s, with the rise of neuroimaging, research shifted its focus from defining cognitive impairment in MS patients to also studying its possible causes. Parallel to the growing interest in the cognitive symptomatology of MS disease, cognitive rehabilitation programs began to be developed, with the aim of training or recovering affected functions in these patients. Likewise, neuroimaging techniques have played a crucial role in the study of the efficacy of cognitive rehabilitation programs.

The cognitive trainings used in MS patients target multiple cognitive domains that are frequently affected in these patients (Prosperini \& Filippo, 2019). Nevertheless, previous studies have highlighted that cognitive rehabilitation programs targeting specific cognitive domains could maximize their effectiveness (Mitolo et al., 2015). For that reason, we have focused on working memory function (WMF), which is a critical function in daily life, that refers to the capacity to retain information, but also to manipulate and transform it, in order to plan and guide our behaviors. Furthermore, by training WMF we are intrinsically training information processing speed (IPS), a central alteration in this clinical population and also closely related to WMF.

In this regard, we have used an intensive adaptive working memory training, and we have assessed its efficacy by measuring changes in participants' performance and different variables obtained using functional magnetic resonance imaging (fMRI), that is, brain activity and functional connectivity (FC). To train WMF, we have selected the n-back paradigm, which is the most popular WMF task. It is used extensively in neuropsychological and neuroimaging research, and it has been tested with good results in healthy controls and clinical populations (Dobbs \& Rule, 1989; Jaeggi et al., 2010; Owen et al., 2005; Redick \& Lindsey, 2013; Wager \& Smith, 2003).

Finally, in this thesis, we attempt to address some of the methodological problems associated with working with MS patient samples. In this regard, MS is a heterogeneous disease with different clinical manifestations and, by extension, different responses to therapies (including cognitive therapies). Therefore, we have tried to select a homogenous group of patients in terms of the clinical evolution of the disease. Furthermore, as mentioned above, we have focused our therapy on one cognitive domain, in contrast to previous studies that focused the cognitive rehabilitation program on different cognitive domains. Last but not least, we have to consider the sample size, especially when we are working with a clinical population. Due to the difficulty of recruiting MS patients, we have used a powerful experimental design to partially counteract this problem. Hence, our study includes a group of healthy control participants and a group of MS patients. Both groups were subdivided into untrained groups and trained groups, in order to control a wide range of differences between groups and intra groups. 


\section{THESIS OUTLINE}

This thesis is divided into three studies that are briefly summarized in this initial description:

\section{Study I}

In the first study, we have explored the effect of the WM-lesion location on FC in the MS patients included in the study, in order to explore the relationship between alterations in FC and cognitive performance. It is important to note that the participants recruited for this investigation consist of a homogeneous group of patients of the RRMS subtype, with no cognitive impairment and in the early stages of the disease. In fact, their cognitive performance does not differ from that of the HC included in the study. Therefore, it is particularly relevant to study whether the changes in FC associated with WM-lesions are related to maladaptive processes or to compensatory mechanisms.

\section{Study II}

In this second study, we explored the effectiveness of a specific WMF training program in MS patients compared to $\mathrm{HC}$, using an n-back task. For this purpose, we studied changes in task performance measured by correct responses (CRs) and reaction times (RTs), and we studied the changes in brain activity associated with the cognitive training. The objective of this study was, on the one hand, to address the hypothesis that programs targeting specific cognitive domains maximize their effectiveness and, on the other hand, to study the neural plasticity mechanisms associated with a training focused on one of the most affected cognitive functions in MS patients, namely WMF.

\section{Study III}

In the last study, we explored neural plasticity processes associated with the WMF training from an FC approach. The brain is not a structure divided into regions that are activated or deactivated individually, but rather these regions are interconnected, forming functional networks. Just as the study of the activation of specific regions allows us to understand how certain brain plasticity mechanisms work and the most relevant brain regions associated with the cognitive training used, the study of how the connections between these regions are affected gives us a broader view of how cognitive training can affect the functioning of the brain as a whole. 


\section{CHAPTER 1}

\section{GENERAL INTRODUCTION}




\section{BACKGROUND}

\section{Multiple sclerosis}

Multiple sclerosis (MS) is a chronic, autoimmune, and neurodegenerative disease of the central nervous (CNS) system that is characterized by widespread lesions in the brain and spinal cord. MS results in motor, cognitive, and neuropsychiatric symptoms (Chiaravalloti \& DeLuca, 2008). MS is the most common cause of non-traumatic neurological disability in young adults in Spain (PérezCarmona et al., 2019) and many other countries (Browne et al., 2014) .The onset of this disease occurs between the ages of $20-40$ in $70 \%$ of the cases, and it is more common in women, with a female to male sex ratio of 2.5 to 1 (Federation, 2020; Ohlmeier et al., 2020). Furthermore, recent serial cross-sectional assessments have reported an increasing incidence of MS in women (Magyari, 2016; Walton et al., 2020).

The worldwide prevalence of MS has increased 30\% since 2013, reaching 2.8 million cases in 2020 (Walton et al., 2020). In Spain, it is estimated that 47000 persons are affected by MS, and about 1800 new cases are diagnosed each year (Sociedad Española de Neurología, 2016). Recent epidemiological studies indicate that MS cases in Spain show an upward trend in recent years, as well as the female to male sex ratio (Costa-Arpín et al., 2020; Perez-Carmona et al., 2019).

Historically, in the beginning of the 20th century, MS was considered a predominantly male disease (Brain, 1930), probably because at that time the role of breadwinner was associated with men. Therefore, a disease that was thought considered to primarily affect physical functionality conflicted with that role. Consequently, MS would be diagnosed more frequently in men than in women. However, as research progressed, it was revealed that this disease was not only a motor disorder, but it also included a broad range of clinical manifestations, including sensory, cognitive, and neuropsychological alterations (Compston \& Coles, 2008; Murphy et al., 2017). Additionally, epidemiological studies have reported an increase in the female/male ratio over time, although the underlying cause of the growing female preponderance remains unclear (Magyari, 2016). In this regard, the speed of the changes suggests environmental factors acting at the population level as well as possible epigenetic modification of HLA-DRB1*1501 (Chao et al., 2009).

Although more than a century has passed since Charcot, Carswell, and others described the clinical and pathological characteristics of MS, the etiology and pathogenesis of this disease are not yet definitively known (Noseworthy et al., 2000). Additionally, the clinical course of MS is highly variable. Even if patients can be classified into different phenotypes, the course and symptomatology 
is highly variable even within groups (Disanto et al., 2011). The motor symptoms involve, among others, limb weakness, paresis, spasticity, tremor, ataxia, dysarthria eye movement disorders, and fatigue (Compston et al., 2005). Sensory symptoms include numbness in one or more limbs, paresthesia, visual alterations such as optic neuritis, internuclear ophthalmoplegia diplopia, or changes in visual acuity, as well as olfactory, taste, auditory, or visual loss, chronic pain syndromes, and L'hermitte sign, which involves a sensation like an electric shock in the back and limbs on flexing the neck (Bobholz \& Gremley, 2011). Affected aspects of cognitive functioning in MS (which will be described in detail below) include attention, information processing efficiency, executive functioning, processing speed, and long-term memory (Chiaravalloti \& DeLuca, 2008).

\section{Diagnosis}

Diagnostic criteria for multiple sclerosis combining clinical, imaging, and laboratory evidence have evolved over time. Although there are several clinical criteria for the diagnosis of MS, the most commonly used are the McDonald criteria (McDonald et al., 2001). These criteria have been periodically reexamined and updated on several occasions based on to new data, emerging technology, and evolving consensus (Polman et al., 2005, 2011). The following tables include the most recently revised criteria, the 2017 McDonald criteria (Thompson et al., 2018), for patients presenting a clinical relapse at onset (Table 1) and for patients presenting progression from onset (Table 2). 
Table 1. The 2017 McDonald criteria for diagnosis of multiple sclerosis in patients with an attack at onset.

\begin{tabular}{|c|c|c|}
\hline $\begin{array}{l}\text { Clinical } \\
\text { attacks }\end{array}$ & $\begin{array}{l}\text { Number of lesions with objective } \\
\text { clinical evidence }\end{array}$ & $\begin{array}{l}\text { Additional data needed for a diagnosis of } \\
\text { multiple sclerosis }\end{array}$ \\
\hline$\geq 2$ & $\geq 2$ & None $^{\mathrm{a}}$ \\
\hline$\geq 2$ & $\begin{array}{l}1 \text { (as well as clear-cut historical } \\
\text { evidence of a previous attack } \\
\text { involving a lesion in a distinct } \\
\text { anatomical location }^{\text {b }} \text { ) }\end{array}$ & None $^{\mathrm{a}}$ \\
\hline$\geq 2$ & 1 & $\begin{array}{l}\text { Dissemination in space demonstrated by: } \\
\text { - An additional clinical attack implicating a } \\
\text { different CNS site. } \\
\text { - OR or by MRI }{ }^{\mathrm{c}}\end{array}$ \\
\hline 1 & $\geq 2$ & $\begin{array}{l}\text { Dissemination in time demonstrated by: } \\
\text { - An additional clinical attack } \\
\text { - OR by MRI }{ }^{\mathrm{d}} \text { OR demonstration of CSF- } \\
\text { specific oligoclonal bands }\end{array}$ \\
\hline 1 & 1 & $\begin{array}{l}\text { Dissemination in space demonstrated by: } \\
\text { - An additional clinical attack involving a } \\
\text { different CNS site } \\
\text { - OR by MRI } \\
\text { AND } \\
\text { Dissemination in time demonstrated by: } \\
\text { - an additional clinical attack } \\
\text { - OR by MRI }{ }^{\mathrm{d}} \text { OR demonstration of CSF- } \\
\text { specific oligoclonal bands }\end{array}$ \\
\hline
\end{tabular}

${ }^{a}$ However, MRI is highly recommended. In addition, spinal cord MRI or CSF examination should be considered in patients with insufficient clinical and MRI evidence supporting multiple sclerosis, with a presentation other than a typical clinically isolated syndrome, or with atypical features.

${ }^{b}$ In the absence of documented objective neurological findings, this can include historical events with symptoms and evolution characteristic of a previous inflammatory demyelinating attack; at least one attack, however, must be supported by objective findings.

${ }^{c}$ Dissemination in space can be demonstrated by one or more T2-hyperintense lesions ${ }^{*}$ that are characteristic of multiple sclerosis in two or more of four areas of the CNS: periventricular, cortical or juxtacortical, and infratentorial brain regions, and the spinal cord

${ }^{d}$ Dissemination in time can be demonstrated by the simultaneous presence of gadolinium-enhancing and nonenhancing lesions at any time or by a new T2-hyperintense or gadolinium-enhancing lesion on follow-up MRI, with reference to a baseline scan, irrespective of the timing of the baseline MRI. Unlike the 2010 McDonald criteria, no distinction between symptomatic and asymptomatic MRI lesions is required.

${ }^{e}$ The presence of CSF-specific oligoclonal bands does not demonstrate dissemination in time per se but can substitute for the requirement for demonstration of this measure. 
Table 2. The 2017 McDonald criteria for diagnosis of multiple sclerosis in patients with disability progression from onset.

$\geq \mathbf{1}$ year of disability progression (retrospectively or prospectively determined) independent of clinical relapse

\section{Plus two of the following criteria}

- One or more T2-hyperintense lesions* characteristic of multiple sclerosis in one or more of the following brain regions: periventricular, cortical or juxtacortical, or infratentorial

- Two or more T2-hyperintense lesions* in the spinal cord

- Presence of CSF-specific oligoclonal bands

*Unlike the 2010 McDonald criteria, no distinction between symptomatic and asymptomatic MRI lesions is required.

\section{Phenotypes}

Two medical conditions have been related to the prodromal phase of MS. On the one hand, the clinically isolated syndrome $(C I S)$ is defined as a first symptomatic episode with characteristics of inflammatory demyelination, that has not demonstrated the dissemination in time required for the diagnosis of MS. Approximately $85 \%$ of MS patients present with a CIS and later develop the relapsing-remitting MS form (Disanto et al., 2011). On the other hand, in the radiologically isolated syndrome (RIS) the patient presents without overt clinical symptoms but with MRI findings highly suggestive of MS. Typically, one-third of patients convert to clinically definite MS within 5 years, with some progressing directly to primary-progressive MS (Yamout \& Al Khawajah, 2017).

Four MS phenotypes have been defined according to the distinct clinical courses observed in the disease (Bobholz \& Gremley, 2011; Forn, 2020):

Relapsing-remitting MS (RRMS) is characterized by unpredictable relapses, followed by periods of remission lasting as long as several years with no new signs of disease activity, and it describes the initial course of $80 \%$ of individuals with MS. There is a version of this phenotype called "Benign $M S$ " characterized by very mild attacks separated by long periods with no symptoms, followed by a full recovery.

Secondary-progressive MS (SPMS) is characterized by a gradual worsening of the symptoms with or without occasional relapses or minor remissions. Following the natural course of the RRMS phenotype, about half of patients develop into SPMS within 10 years, and in up to $90 \%$ of RRMS 
patients, within 20-25 years. The use of disease-modifying therapies may affect these transition rates, but this has not been quantified (Gross \& Watson, 2017; Weinshenker et al., 1989).

Primary-progressive $M S(P P M S)$ involves a continuous gradual worsening of the symptoms without clear attacks. This course type describes approximately $10 \%$ of individuals who never had remission after their initial MS symptoms (Bobholz \& Gremley, 2011). This subtype is more frequent in people who are older at the disease onset.

Progressive-relapsing MS (PRMS) is characterized by a progressive decline after the onset of the disease, as well as PPMS but with some acute periods of symptom relapse. There might or might not be recovery from these acute periods, and the period between relapses shows continued progression of the disease.

Relapsing-Remitting MS

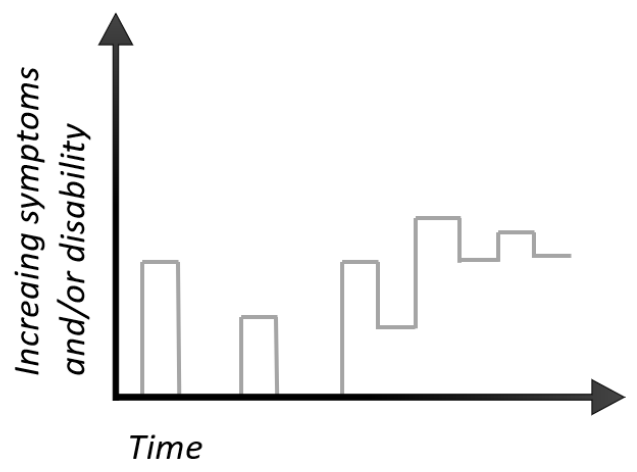

Primary-Progressive MS

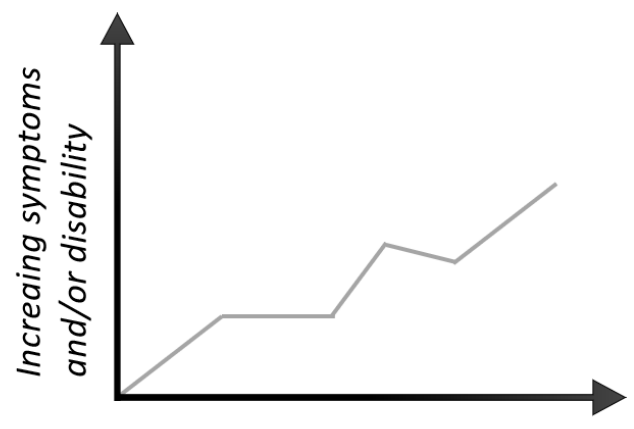

Time
Secondary-Progressive MS

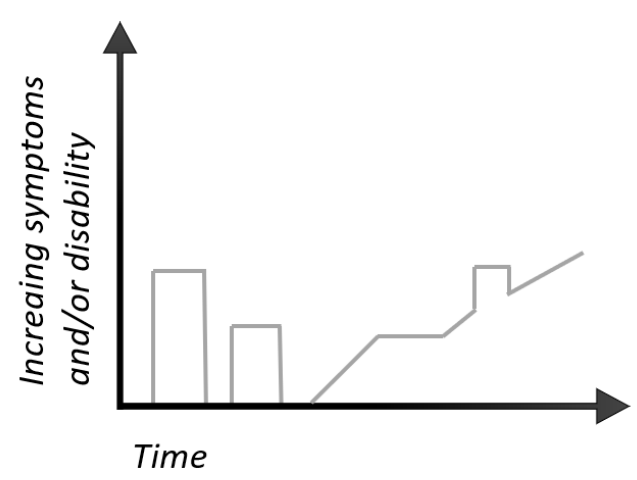

Progressive-Relapsing MS

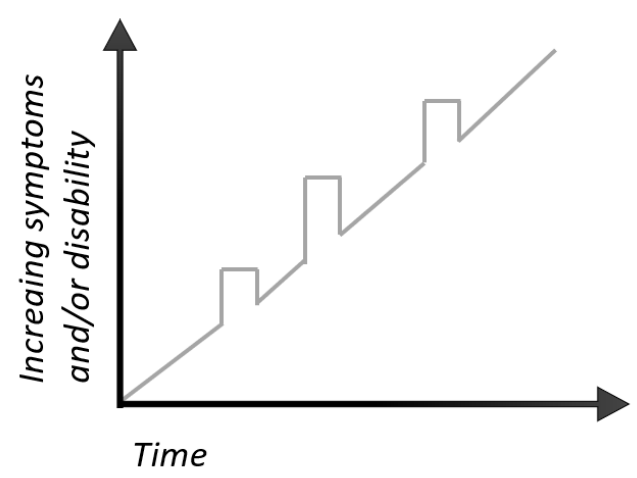

Figure 1. Multiple sclerosis phenotypes. 


\section{Etiology}

Although the etiology and pathogenesis of MS have been extensively investigated, no definite cause of MS has been found. Regarding the etiology, there are many theories, such as viral infection, or that retroviral reactivation primes a susceptible immune system for an abnormal reaction later in life, or that MS is a response to a chronic viral or bacterial infection (Institute of Medicine, 2001; Bobholz \& Gremley, 2011). The precise cause of MS is not known; nevertheless, both genetic and environmental factors appear to influence susceptibility to developing the disease.

\section{Genetic factors}

MS is not considered a genetic disease in the classic sense; however, studies with siblings have demonstrated that genetic risk factors play a principal pathogenic role in MS etiology (Institute of Medicine, 2001). The risk of developing MS in the general population is approximately $0.1 \%$, whereas it is between 25\% and 30\% for monozygotic twins (Sadovnick et al., 1993; Willer et al., 2003) and approximately 5\% for dizygotic twins and other siblings (Compston \& Coles, 2008). Interestingly, the risk is double (12.2\%) in the case of child with two parents who have MS (Ebers et al., 2000), compared to dizygotic twins and siblings, whereas the risk decreases markedly (2\%) in the case of only one parent and second-degree and third-degree relatives with the disease (1\%) (Compston $\&$ Coles, 2008). A summary of these results can be found in Figure 2.

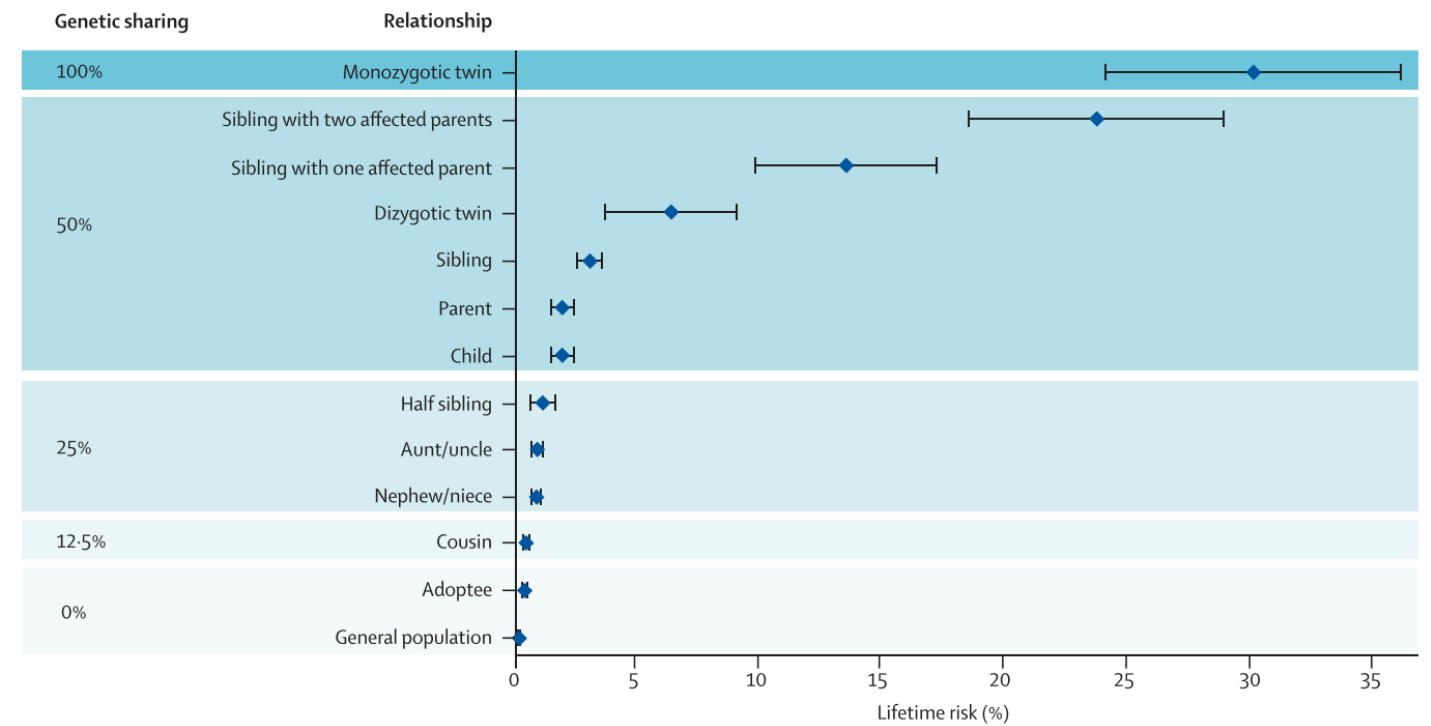

Figure 2. Recurrence risks for multiple sclerosis in families, extracted from (Compston \& Coles, 2008). Age-adjusted recurrence risks for different relatives of probands with multiple sclerosis, and degree of genetic sharing between relative and proband. Pooled data from population-based surveys. Error bars indicate the estimated $95 \%$ confidence intervals. 
As mentioned above, the etiology and precise mechanisms of the MS disease process are not yet fully understood. However, current knowledge suggests that MS involves an autoimmune process in which the immune system abnormally directs itself against CNS axons via the activation of T cells that target the myelin sheath (Bishop \& Rumrill, 2015). In fact, more than a hundred genetic regions have been associated with MS, and most of them are involved in immunological system functions (Dendrou et al., 2015). Available data are consistent with a polygenic model in which risk is defined by a single moderate-effect allele (odds ratio near 3 or 4) and several alleles with a much smaller effect (odds ratio < 1.5) (Leray et al., 2016). The family of genes encoding human leukocyte antigens (HLA) have shown a strong association with MS and been the focus of research for decades (Jersild et al., 1973; Naito et al., 1972; Olerup \& Hillert, 1991). HLA class I and class II genes encode the products that present antigens to $\mathrm{CD} 4+\mathrm{T}$ and $\mathrm{CD} 8+\mathrm{T}$ lymphocytes, respectively. A recent study reported that HLA-DRB1*04:05, DRB1*15:01, and DPB1*03:01 correlated with MS susceptibility, and DRB1*01:01, DRB1*09:01, DRB1*13:02, and DPB1*04:01 protected against MS (Watanabe et al., 2021). In addition, polymorphisms with IL7R and ILRA genes were also modestly associated with MS (Leray et al., 2016).

\section{Environmental factors}

In the last few decades, there has been a noticeable increase in MS in most countries; although genes have not changed in this time, lifestyle and environment have (Alfredsson \& Olsson, 2019). Therefore, environmental factors should be considered to have a strong influence on the immune repertoire (Brodin et al., 2015).

Among the infectious agents suggested to be involved in developing MS, the Epstein-Barr virus (EBV) has been widely and repeatedly reported (Alfredsson \& Olsson, 2019; Bishop \& Rumrill, 2015; Handel et al., 2010; Leray et al., 2016; Olsson et al., 2016). Although it has not been possible to establish any causal relationship between MS and EBV, the risk of developing MS is double in individuals who have had clinically overt infectious mononucleosis (Handel et al., 2010).

Regarding lifestyle, smoking showed a clear dose-response relationship, where the cumulative of smoking dose is related to an increased risk of MS (Ghadirian et al., 2001; Hedström et al., 2009). This factor, along with EBV, has shown the strongest consistent evidence of an association (Belbasis et al., 2015). Furthermore, not only active smoking increases the risk of MS, but passive smoking has also been associated with increased risk of MS. This suggests that even minor "lung-irritation" may be important (Hedström et al., 2011) Therefore, air pollution has also been considered a trigger of CNS neuroinflammation (Heydarpour et al., 2014), although replication studies are required. In 
addition, adolescent obesity has been associated with MS (Hedström et al., 2016; Langer-Gould et al., 2013; Munger et al., 2013). Obesity is characterized by "low grade" inflammation in which increased levels of proinflammatory mediators are produced in the fat tissue (Lumeng et al., 2007; Procaccini et al., 2015), and it also leads to decreased bio-availability of vitamin D, which can lead to a proinflammatory bias (Wortsman et al., 2000).

Geographical location is one of the most widely studied environmental factors, especially associated with latitude and sun exposure/vitamin D (Koch-Henriksen \& Sørensen, 2010; Simpson et al., 2019; Walton et al., 2020). Latitude is related to vitamin D, not only because of the number of hours of sunshine in a specific geographical region, but also because we depend on ultraviolet radiation to convert vitamin D to an active metabolite (Lucas et al., 2015). Increasing vitamin D levels and sun exposure, especially before age 20, have been associated with a decreased risk of MS (Bjørnevik et al., 2014; Cortese et al., 2015; Kampman et al., 2007; Munger et al., 2006). Specifically, high levels of serum 25(OH)D prior to MS clinical onset is associated with a reduced risk of MS (Munger et al., 2006; Salzer et al., 2012), and 25(OH)D deficiency has been associated with an increased risk of MS (Munger et al., 2017). However, this finding is not undisputed because genetically determined low $25(\mathrm{OH}) \mathrm{D}$ has been associated with an increased risk of both adult and pediatric onset of MS (Gianfrancesco et al., 2017; Mokry et al., 2015; Rhead et al., 2016). Linked to this, the global distribution of MS can be generally defined as increasing with the distance from the equator (Compston \& Coles, 2008). Figure 3 shows a map of the worldwide distribution of MS disease, extracted from the Atlas of MS created by the International MS Federation (https://www.atlasofms.org/), which is the most extensive worldwide study of the epidemiology of MS (Walton et al., 2020). 


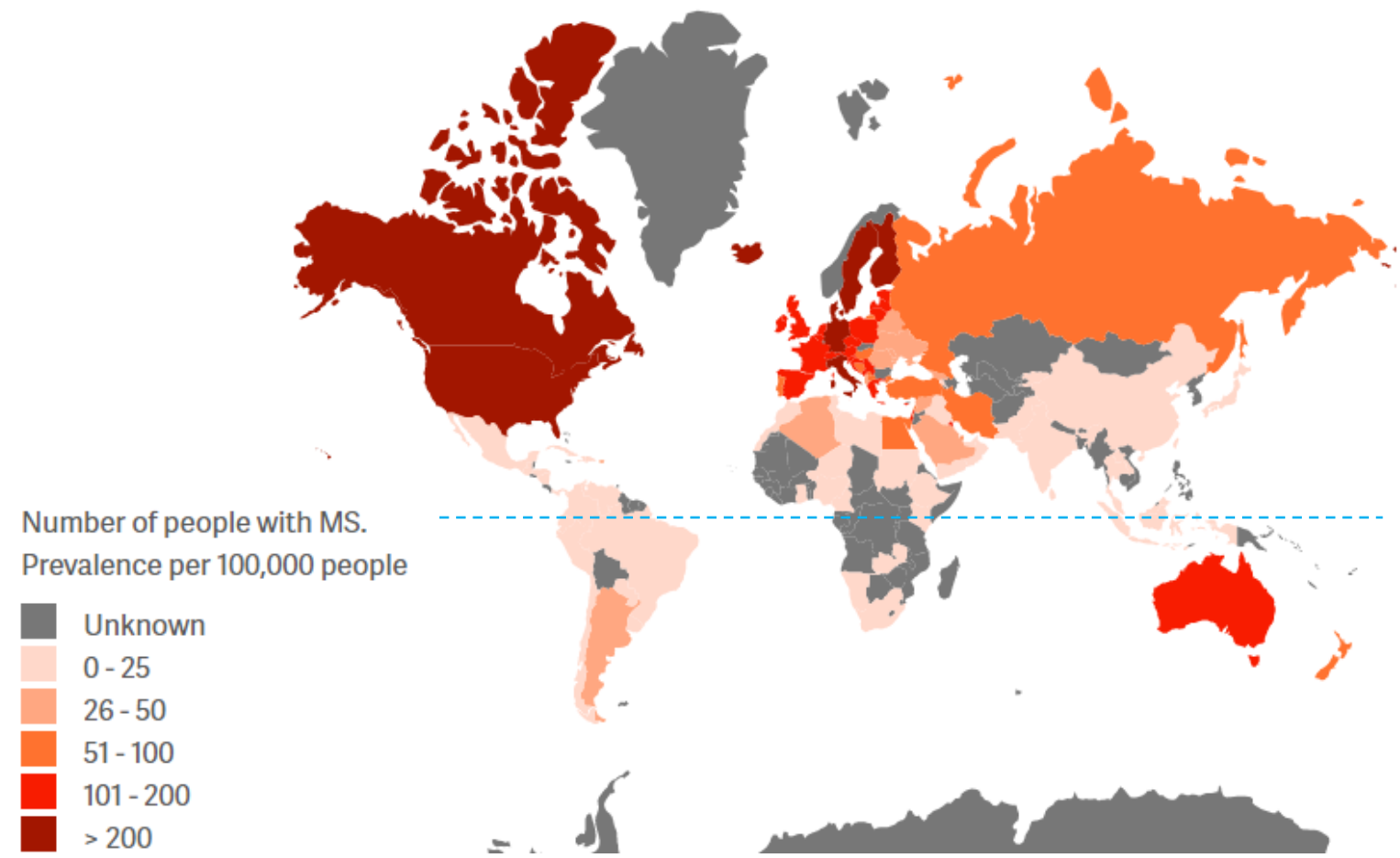

Figure 3. MS prevalence worldwide extracted from the Atlas of MS (https://www.atlasofms.org/). The blue dashed line corresponds to the equator line.

However, understanding MS prevalence as only associated with the latitude gradient conceals many places with disproportionately high or low frequencies that have been associated with genetic factors (Detels et al., 1977; Fawcett \& Skegg, 1988; Grønlie et al., 2000; Skegg et al., 1987). Charles M. Poser proposed the "Viking theory" in 1995, which states that MS is closely linked to Scandinavian genes, and the worldwide distribution of this disease is related to the Viking invasions and migrations from Scandinavian countries to the rest of the world (Poser, 1995). However, although the importance of genetic factors has been demonstrated, migration studies indicate that the relevance of environmental factors should not be underestimated. It has been observed that individuals who migrate from a low-risk country to a high-risk country before adolescence show a similar risk of developing MS as those who are born and reside in the high-risk country (Ahlgren et al., 2010, 2012; Berg-Hansen et al., 2015; Gale \& Martyn, 1995; Rotstein et al., 2019). 


\section{Neuropathology}

MS is traditionally considered an autoimmune-mediated demyelinating disease, characterized by CNS inflammation, oligodendrocyte loss, demyelination, and axonal degeneration, the pathoetiology of which is unknown. However, the key question remains whether autoimmunity is the initiator of the disease (outside-in) or the consequence of a slow and as yet uncharacterized cytodegeneration (oligodendrocytosis) that leads to a subsequent immune response (inside-out) (Morgan et al., 2021). In the "outside-in" paradigm, an unknown trigger activates peripheral T- and/or B-cells and leads to an infiltration of these cells in the CNS via an apparently dysfunctional blood brain barrier. T-cells attack myelin in the CNS, causing oligodendrocytes to degenerate, resulting in myelin loss and the release of myelin debris. Innate immune cells engulf the myelin debris and act as antigen-presenting cells to T-cells that then exacerbate the process of oligodendrocyte damage and demyelination. In contrast, in the "inside-out" hypothesis, oligodendrocyte degeneration is initially triggered by internal metabolic dysfunction in the CNS, leading to demyelination and gliosis, with subsequent release of inflammatory cytokines and chemokines. This cascade of events compromises the integrity of the blood brain barrier, resulting in permeability to peripherally circulating T- and Bcells. Within the CNS, T-cells (CD4+ and CD8+) interact with antigen-presenting cells via the major histocompatibility complex and become activated. These activated T-cells also release inflammatory mediators, such as cytokines, nitric oxide, and glutamate, which exacerbate the degenerative process, leading to a subsequent immune response that further accelerates oligodendrocytosis and demyelination (Sen et al., 2020). Both paradigms have obtained experimental and clinical support to date; therefore, they could be considered complementary rather than competitive hypotheses (Morgan et al., 2021; Titus et al., 2020).

The neuropathology of MS comprises a wide variety of lesions, including specific focal lesions, diffuse abnormalities, and brain atrophy.

\section{Focal lesions}

The name multiple sclerosis refers to the numerous scars that form in the CNS (Charcot, 1868). These scars, better known as plaques or lesions, are the pathological hallmark of MS (Popescu et al., 2013). The plaques are multiple focal areas of demyelination within the CNS. These lesions most commonly affect the white matter (WM) in the periventricular, juxtacortical, and infratentorial regions of the brain, as well as in the spinal cord (Barkhof et al., 1997; Martínez-Heras et al., 2020). However, MS demyelination lesions are known to also occur in grey matter (GM) (Calabrese et al., 2013), such as the cerebral cortex, deep nuclei, and brainstem. 


\section{White matter lesions}

According to the presence and distribution of myelin-laden macrophages within MS lesions, several plaque subtypes can be distinguished:

a) Acute active lesions: In this acute phase, lesions are infiltrated by lymphocytes, microglia, and $\mathrm{T}$ cells, and macrophages destroy myelin and to a variable degree, oligodendrocytes. Myelin debris is picked up by macrophages and degraded. First, macrophages contain myelin fragments (early degradation products); later, they contain proteins and lipids from chemical degradation of myelin (late degradation products). This type of lesion is infrequent, probably due to its short duration, and they are most often found in early stages of the disease (Agamanolis, 2019; Filippi, Rocca, et al., 2012).

b) Chronic active lesions: These lesions are more sharply circumscribed than the previous ones. Macrophages accumulate especially at the expanding edge of the plaque, with their number declining toward the lesion's inactive center (Filippi, Rocca, et al., 2012).

c) Smoldering lesions: Also known as slowly expanding lesions, they are characterized by a few myelin-laden macrophages present at the plaques' edge (Filippi, Rocca, et al., 2012).

d) Chronic inactive lesions: With time, gliosis develops, and plaques reach a burned-out stage consisting of demyelinated axons traversing glial scar tissue (Agamanolis, 2019). A variable degree of microglia activation is present in the periplaque WM, whereas the number of microglia is profoundly reduced in the center of the demyelinated plaque (Lassmann, 2018).

e) Shadow plaques: Like inactive lesions, these lesions are sharply demarcated from the surrounding normal-appearing WM (Lassmann, 2018; Popescu et al., 2013). If the inflammatory process is arrested in an early phase, plaques are partially remyelinated. In more advanced lesions, remyelination is ineffective because gliosis creates a barrier between the myelin producing cells and their axonal targets. The pathological process may be arrested at any time, sometimes after partial demyelination (Agamanolis, 2019).

Active lesions are most often found in acute and RRMS, and they might be the pathological substrate of clinical attacks (Filippi, Rocca, et al., 2012; Martínez-Heras et al., 2020). Previous studies have reported an association between chronic active lesions and a more aggressive disease evolution (Absinta et al., 2019; Lucchinetti et al., 2000). Indeed, differences in the severity of demyelination, remyelination, and neuroaxonal damage could explain why some patients recover completely from relapses, whereas in others, their disability deteriorates more rapidly. Meanwhile, chronic inactive lesions and smoldering plaques predominate in chronic progressive MS, and slowly expanding 
plaques might contribute to this progression (Filippi, Rocca, et al., 2012). Finally, shadow plaques are as common in progressive MS cases as in RRMS patients because evidence for remyelination is seen in almost half of chronic MS lesions (Popescu et al., 2013).

\section{Gray matter lesions}

As in WM, the pathological hallmark that distinguishes the GM lesions characteristic of MS from brain damage in other diseases of the CNS is the destruction of oligodendrocyte and primary demyelination (Lassmann, 2018). However, in the case of GM, there is a much lower degree of inflammation, edema, microglia activation, and macrophage recruitment (Peterson et al., 2001), whereas the loss of synapses is greater than in the case of WM lesions, associated with a variable degree of loss of axons, neurons, and glial cells (Dutta et al., 2011; Wegner et al., 2006). Furthermore, remyelination is more extensive in the cortex compared to the WM (Albert et al., 2007).

There are different types of GM lesions in MS, and their incidence differs between early relapsing and progressive MS (Bø et al., 2003; Kidd et al., 1999; Peterson et al., 2001). Their classification is based on lesion distribution within cerebral cortex/subcortical WM (Figure 4) (Bo et al., 2006; Lassmann, 2018).

a) Type 1 lesions are present at the cortico-subcortical border and affect the GM as well as the WM.

b) Type 2 lesions are small perivenous intracortical lesions.

c) Type 3 lesions are located in the subpial layers of the cortex. They are the most common cortical lesion type, especially in the progressive stage of the disease. They are mainly located in the cortical sulci and the deep invaginations of the brain surface, such as the insular cortex or the cingulate cortex. They are associated with inflammation in the meninges and expand from the pial surface into the deeper cortical layers.

d) Type 4 lesions are a special type of cortical Type 3 lesions that affect the entire cortex but do not pass the border between the cortex and the WM. 


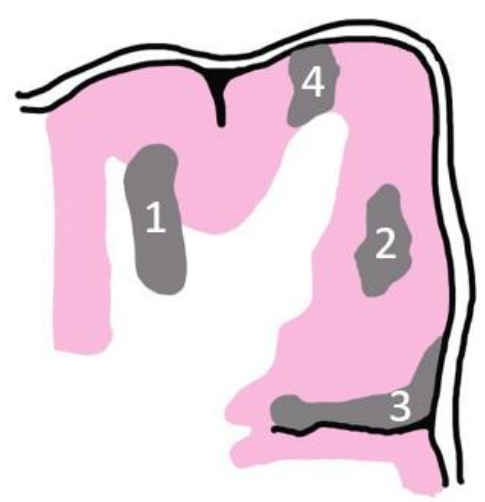

Figure 4. Gray matter lesions types, adapted from (Bo et al., 2006). Numbers match each cortical lesion type.

\section{Normal-appearing brain tissues}

As previously mentioned, focal lesions constitute the pathological hallmark of MS. However, diffuse abnormalities in WM and GM brain tissues have also been reported (Kutzelnigg et al., 2005; Vrenken $\&$ Geurts, 2007), and they are more frequent in progressive MS cases (Lassmann, 2018). They are characterized by perivascular inflammatory infiltrates, moderate brain edema, diffuse microglia activation, diffuse axonal injury, and astrocytic gliosis (Kutzelnigg et al., 2005). These pathological abnormalities are secondary to axonal and neuronal damage within focal lesions. This reduction in axons is attributable to both anterograde, so-called Wallerian, and retrograde degeneration (Dziedzic et al., 2010; Filippi, Rocca, et al., 2012). Regarding retrograde degeneration, the accumulation of phosphorylated neurofilament within the cytoplasm of affected neurons is a good indicator of the severity of this process (Haider et al., 2016). Importantly, diffuse changes in the normal-appearing WM and GM also develop independently from focal lesions, showing a moderate correlation with inflammation in the meninges covering the spinal cord or the cortex (Androdias et al., 2010; Haider et al., 2016).

\section{Brain atrophy}

A neurodegenerative process in both WM and GM tissues takes place is developed in parallel with or as a consequence of the inflammatory phenomenon in patients with MS, in all disease phenotypes (DeLuca et al., 2015; Sastre-Garriga et al., 2017). Brain atrophy seems to proceed at a faster pace in GM than in WM (Dalton, 2004; Sastre-Garriga et al., 2005; Tiberio et al., 2005; Valsasina et al., 2005). In addition, the GM volume loss is not uniformly widespread throughout the brain, but rather 
the atrophy is found in specific GM regions, such as the thalamus, basal ganglia bilaterally, the precentral and postcentral regions bilaterally, and the cingulate bilaterally (Lansley et al., 2013).

\section{Neuropathology and cognitive decline in multiple sclerosis}

MRI plays a central role in the scientific investigation and clinical management of MS. This technique not only supports diagnosis, but it also provides a wide variety of structural and functional methods to study pathological mechanisms of the disease and their relationship with physical and cognitive impairment.

Regarding the results on macroscopic WM lesions, a mismatch between the number and volume of WM lesions and clinical outcomes has been widely reported (Fulton et al., 1999; Heesen et al., 2010; Hulst et al., 2013, 2014; Mollison et al., 2017; Uher et al., 2014). This phenomenon is known as the clinical-radiological paradox (Barkhof, 1999, 2002; Roosendaal et al., 2010), and it is characteristic of studies that mainly focus on the relationship between cognitive impairment and WM lesion burden or lesion number in MS. A recent meta-analysis (Tahedl et al., 2018) highlighted the importance of approaching this relationship from different methodological approaches, such as the location perspective. The presence of lesions in specific WM tracts could provide valuable information to fully understand the relationship between cognitive impairment and WM lesions. However, it has been studied very little (Droby et al., 2016), probably because of the methodological difficulty due to MSlesions occurring at multiple sites.

Cortical lesions have been studied less due to technical limitations to detect them; nevertheless, these lesions are more clearly related to cognitive impairment in MS (Rinaldi et al., 2010). Patients with cortical lesions have significantly lower scores than those without cortical lesions (Curti et al., 2018; Shinoda et al., 2020). A longitudinal study found that cortical lesion load characterizes patients with MS, with those with the most severe GM atrophy having the worst physical and cognitive prognosis (Calabrese et al., 2012). The MS patients who showed a high cortical lesion load at baseline showed the worse clinical evolution and a significant progression of cortical atrophy after 5 years.

Moreover, whole WM and GM volume loss has been clearly linked to neuropsychological performance and neuropsychiatric symptoms in MS patients (Lazeron et al., 2006; Sanfilipo et al., 2006). Furthermore, the presence of atrophy in WM is closely related to lesion formation and seems to have a major impact on disability, in contrast to GM (Sastre-Garriga et al., 2005). In addition, normal-appearing WM is a significant contributor to cognitive impairment in MS (Abel et al., 2020; Filippi et al., 2000; Rovaris et al., 2002). In contrast to WM atrophy, diffuse WM lesions show higher 
correlations with cognitive impairment than with physical disability (Rovaris et al., 1998; SwirskySacchetti et al., 1992; Yu et al., 2012).

\section{Key brain regions}

Thalamus

The thalamus is a "relay station" structure involved in a wide range of cognitive functions such as attention, memory, executive functioning, and emotion (Minagar et al., 2013). Thalamic axons transmit information between a number of subcortical and specific cortical areas. Thus, damage to the thalamic nuclei and their connections potentially impairs a wide range of neurological functions that may clinically translate into significant cognitive and mental disability in MS (Amin \& Ontaneda, 2021; Cifelli et al., 2002; Houtchens et al., 2007; Vercellino et al., 2005). Several studies have reported the importance of thalamic abnormalities in all the different MS disease types (Mesaros et al., 2011; Ramasamy et al., 2009; Rocca, Mesaros, et al., 2010; Sepulcre, Sastre-Garriga, et al., 2006; Wylezinska et al., 2003). Specifically, thalamic atrophy has been associated with disability, cognitive impairment, and fatigue (Amin \& Ontaneda, 2021; Calabrese, Rinaldi, Grossi, et al., 2010; Heesen et al., 2010). Based on thalamic pathology, cognitively preserved MS patients can be distinguished from cognitively impaired MS patients (Schoonheim, Hulst, et al., 2015). Among the cognitive functions, thalamic atrophy is closely related to attention and processing speed deficits (Bergsland et al., 2016; Bisecco et al., 2018; Rojas et al., 2018).

\section{Hippocampus}

This hippocampus is a sub-cortical region associated with memory function. It is known to be involved in cognitive decline in both healthy controls (HC) and patients with neurodegenerative disease, especially Alzheimer's disease patients (Aguirre, Costumero, et al., 2019; Calabrese, Rinaldi, Mattisi, et al., 2010; Hulst et al., 2015; Jaroudi et al., 2017; Squire \& Zola-Morgan, 1991). In MS, hippocampal atrophy has been found in the RRMS, SPMS, and PPMS phenotypes, and it has been associated with poorer performance on a verbal memory test (Anderson et al., 2010; Sicotte et al., 2008). Furthermore, atrophy of hippocampal subfields has been related to episodic memory impairment, making it possible to discriminate between cognitively preserved and cognitively impaired MS patients (González Torre et al., 2017). Additionally, increased hippocampal activation has been associated with cognitive preservation, whereas cognitive impairment has been associated with decreased hippocampal activation (Hulst et al., 2015). 


\section{Corpus callosum}

The corpus callosum is the largest white-matter tract connecting the two hemispheres. This structure is notably affected in MS (Evangelou et al., 2000; Welton et al., 2015), and it is the most consistent difference observed between MS and healthy controls in DTI studies (Manca et al., 2018). Corpus callosal atrophy in MS patients correlates with cognitive impairment, specifically IPS affection measured by the Symbol Digit Modalities Test (SDMT) and Paced Auditory Serial Addition Test (PASAT) (Barkhof et al., 1998; Bergendal et al., 2013; Mazerolle et al., 2013; Sánchez et al., 2008; Sbardella et al., 2015; Yu et al., 2012) and motor impairment measured by the Expanded Disability Status Scale (EDSS) (Juha et al., 2007; Schreiber et al., 2001; Yaldizli et al., 2010). Furthermore, a 17-year longitudinal study reported that corpus callosum atrophy is more aggressive in the first stages of the disease, and it is strongly associated with IPS impairment and disability. Therefore, the integrity of this structure could be considered a suitable biomarker for disability and cognitive function in MS (Granberg et al., 2015; Rimkus et al., 2011; Vaneckova et al., 2012).

\section{Profile of cognitive impairment in MS}

Deficits in several cognitive functions have been reported in MS patients, including memory, executive functions, attention, WMF, and verbal fluency (Benedict et al., 2006; Deloire et al., 2011; Rao et al., 1991). However, slowed IPS seems to be the central deficit in MS (Chiaravalloti \& DeLuca, 2008; Einarsson et al., 2006; Zakzanis, 2000), and it especially affects attention and WMF. Furthermore, slowed IPS and episodic memory decline are considered the most common cognitive deficits in MS (Bergendal et al., 2007; Chiaravalloti \& DeLuca, 2008; Janculjak et al., 2002; Langdon, 2011).

\section{Information processing speed, attention and working memory}

These concepts are closely linked, and so it is challenging to distinguish the tasks of these different domains (Grzegorski \& Losy, 2017; Kujala et al., 1995). The WMF refers to the ability to maintain and manipulate information in the brain for a short period of time, whereas IPS refers to the speed with which one can process this information. Attention is a cognitive function that links different processes such as alertness and vigilance. Attentional deficits can be found in approximately 12-25\% of MS patients (Guimarães \& Sá, 2012; Winkelmann et al., 2007), and complex attention tasks (sustained attention, divided attention, selective attention) are typically impaired. Deficits in these functions contribute to the presence of impairment in other cognitive domains (Grzegorski \& Losy, 2017; Van Schependom et al., 2015). 
With regard to the above, the SDMT, from the Brief Repeatable Battery for Neuropsychological evaluation (BRB-N), is one of the most targeted and sensitive tests, and performance on this test has been found to be impaired in both RRMS and PPMS patients (Benedict et al., 2006; Camp et al., 1999; Deloire et al., 2005; Forn et al., 2009; Nocentini et al., 2006). This test has been demonstrated to be a better screening test for the detection of cognitive impairment in MS than other cognitive tests, even in CIS patients (Deloire et al., 2006; Parmenter et al., 2007). IPS impairment is usually inferred by measuring reaction times (RTs). Indeed, it has been shown that the difference between $\mathrm{HC}$ and MS patients' RTs increases when cognitive task complexity increases (increase in processing demand or cognitive load) (Bonnet et al., 2010; Fittipaldi-Márquez et al., 2017; Reicker et al., 2007). This difference could even be significant for the highest level of cognitive demands, suggesting a saturating effect of cognitive load on cognitive efficiency in MS patients (Bonnet et al., 2010). Aspects related to cognitive efficiency have commonly been studied using the PASAT (Benedict et al., 2004; Deloire et al., 2005; Forn et al., 2008, 2011) and n-back task (Covey et al., 2011; Hubacher, DeLuca, et al., 2015; Parmenter et al., 2006).

\section{Memory}

Long-term memory refers to the ability to learn new information and recall that information at a later time point. It is the most consistently affected cognitive domain in MS patients (DeLuca et al., 1994; Rao et al., 1991). This function is assessed as the amount of information of several types (such as visual or spatial information) learned and recalled. In MS, two separate deficits have been reported (Chiaravalloti \& DeLuca, 2008): first, encoding deficits related to dysfunction of the frontal and subcortical axis; and second, consolidation deficits, associated with mesial temporal lobe dysfunctions and hippocampus atrophy (Benedict et al., 2009; González Torre et al., 2017). Although the primary problem has been associated with encoding deficits (Trenova et al., 2016), this assumption is controversial (Chiaravalloti \& DeLuca, 2008; Islas \& Ciampi, 2019; Thornton et al., 2002).

\section{Executive Function}

Executive functioning comprises all the cognitive skills that are crucial for complex goal-directed behavior and the ability to adapt to the changes or demands of the environment (Chiaravalloti \& DeLuca, 2008). Deficits in executive functions are less frequent than in the previous cognitive domains, occurring in 17-19\% of MS patients (Chiaravalloti \& DeLuca, 2008; Drew et al., 2008; Rao et al., 1991). As mentioned above, executive functions are dependent on IPS because most of the tests used to assess them imply IPS integrity (Islas \& Ciampi, 2019; Leavitt, Wylie, Krch, et al., 2014). The difficulty in assessing a specific domain, such as executive function, may be extrapolated to all 
other domains, but patients usually struggle with managing multiple goals simultaneously (Chiaravalloti \& DeLuca, 2008; Grzegorski \& Losy, 2017).

\section{Language}

Language deficits have been studied less than the above-mentioned functions, even though language impairment frequencies between $20 \%$ and $58 \%$ have been reported in MS patients (Ntoskou et al., 2018). Specifically, verbal fluency deficits have consistently been reported in MS (Henry \& Beatty, 2006). Both semantic and phonemic fluency are impaired in MS patients. However, verbal fluency tests are directly influenced by executive functions, and so many of the deficits have been considered to be due to a dysfunctional executive syndrome (Chiaravalloti \& DeLuca, 2008; Grzegorski \& Losy, 2017).

\section{Cognitive assessment}

To assess cognitive impairment in MS, three neuropsychological test batteries are mainly used:

1) Brief Repeatable Battery of Neuropsychological Tests (BRB-N) (Rao \& Cognitive Function Study Group, 1990) : It is the most widely used neuropsychological battery for MS. To assess the cognitive status of MS patients enrolled in the studies in the present thesis, we have used the Spanish version (Sepulcre, Vannotti, et al., 2006). The administration time for this battery is around 30 minutes, and it is composed of the 5 tests most sensitive to detecting cognitive impairment (see Table 3).

Table 3. BRB-N tests and cognitive functions assessed.

\begin{tabular}{ll}
\hline BRB-N tests & Cognitive function assessed \\
\hline SDMT & $\begin{array}{l}\text { IPS, WMF, attention and visual } \\
\text { search }\end{array}$ \\
\hline PASAT & IPS, WMF, and attention \\
\hline Selective Reminding Test (SRT) & Verbal memory \\
SRT Long-Term Storage & Memory encoding \\
SRT Consistent Long-Term Retrieval & Memory storage \\
SRT Delayed Recall & Memory retrieval \\
\hline 10/36 Spatial Recall Test (SPART) & Spatial memory \\
SPART Long-Term Storage & Memory encoding \\
SPART Delayed-Recall & Memory retrieval \\
\hline Word List Generation Test (WLGT) & Verbal fluency \\
\hline
\end{tabular}


2) Minimal Assessment of Cognitive Function in MS (MACFIMS) (Benedict et al., 2002): A 90minute neuropsychological battery composed of seven tests covering five cognitive domains commonly impaired in MS (IPS/WMF, executive functions, learning and memory, and visual-spatial processing). This battery includes the SDMT, PASAT, California verbal learning test-second version (CVLT-II), brief visuospatial memory test-revised (BVMTRR), Delis-Kaplan Executive Function System Sorting Test (D-KEFS ST), and the Controlled Oral Word Association Test (COWA).

3) Brief International Cognitive Assessment for MS (BICAMS) (Langdon et al., 2012): Standardized brief cognitive test created with the aim of being incorporated in everyday patient assessment. It can be completed in 15 minutes and administered by health professionals who are not cognitive specialists. This battery includes the SDMT, the CVLTII, and the BVMTR-R.

\section{Cognitive rehabilitation and cognitive training}

\section{Cognitive rehabilitation}

Cognitive rehabilitation (CR) can be defined as a repetitive behavioral intervention that focuses on the cognitive functions most affected in a disease with the aim of improving the patient's cognitive performance, daily functionality, and quality of life, and promoting positive neurobiological changes (Mitolo et al., 2015). The intervention program can be approached from three different theoretical models (Díez-Cirarda et al., 2018):

1) Restoration of impaired cognitive function by means of repetitive stimulation with the final objective of reaching a preserved level.

2) Optimization of cognitive function that is not completely lost but has reduced efficacy. Therefore, the cognitive function can be improved through the use of preserved cognitive systems.

3) Compensation for an irrecoverable cognitive function, whose neural substrate damage is also too great to recover from. Consequently, it focuses on enhancing other cognitive domains that could compensate for the deficit in the function damaged, and the use of cognitive strategies, such as mnemotechnic rules or/and external aids, such as a daily diary

CR has been extensively used to treat cognitive deficits in MS patients. The programs used have changed over time as a function of knowledge about the cognitive profile of patients (Goverover et al., 2018; Mitolo et al., 2015; O’Brien et al., 2008). The effects of these rehabilitation programs have 
been widely studied from a behavioral perspective, revealing significant cognitive improvements in MS patients (Mitolo et al., 2015). However, recent metanalyses have reported little evidence for the efficacy of these interventions due to several factors, such as unpowered samples, unsuitable allocation, or randomization procedures (das Nair et al., 2016; Goverover et al., 2018; Rosti-Otajärvi \& Hämäläinen, 2014). Beyond these methodological problems, which are difficult to solve due to the characteristics of the sample or the intervention program, CR is a powerful tool for treating the cognitive deficits associated with the disease (Charvet et al., 2017; Goverover et al., 2018; Mitolo et al., 2015).

The effects of rehabilitation programs have not only been studied from a behavioral perspective, but also at the level of neural plasticity mechanisms using imaging techniques, with MRI being the most powerful tool (Prosperini \& Filippo, 2019). Several studies have demonstrated that neural plasticity contributes to the potential for functional recovery despite MS-related damage (Prosperini \& Filippo, 2019; Reddy et al., 2002). In this regard, two main approaches are used to measure plasticity effects of CR in MS patients: structural changes and functional changes. Very few studies have reported significant structural changes after a CR program (De Giglio, Upadhyay, et al., 2016), and several studies failed to find any structural changes following intervention (Campbell et al., 2016; Filippi, Riccitelli, et al., 2012; Parisi, Rocca, Valsasina, et al., 2014). However, relevant functional changes have been reported following cognitive interventions (Prosperini \& Filippo, 2019). According to the methodology used for the analysis of fMRI, the effects can be classified as changes in brain activation or brain connectivity.

The following provides a summary of the state of the research on functional changes, measured with fMRI, associated with CR in MS patients. Despite the promising findings and advances in this field, a number of methodological limitations must be taken into consideration when interpreting the results, such as small sample sizes, selection bias toward less severe MS, no intervention or wait list as control group, and lack of longer-term post-intervention assessments (Prosperini \& Filippo, 2019).

\section{Brain activity changes after cognitive rehabilitation}

The majority of the studies that used fMRI to observe neuroplasticity effects in MS patients after rehabilitation programs have focused on multi-domain interventions, targeting the most affected cognitive functions in MS patients, i.e., attention, IPS, WMF, executive functions, short-term memory, and episodic memory. The results reported in most of them showed increased activation after rehabilitation programs (see Table 4). In this regard, although CR programs are usually multidomain, the effects of these programs on brain activation are usually assessed with tasks that measure 
a specific cognitive domain. Thus, studies can be divided into two main categories: those that assess attention, WMF, and IPS, and those that focus on evaluating learning and memory functions.

In the first category, the PASAT task measures sustained attention, cognitive flexibility, WMF, and IPS. In this regard, two studies investigate changes in brain activity in MS patients with cognitive impairment in cognitive domains specific to of this task. Both studies reported increased brain activity in the cerebellum and parietal lobe (Cerasa et al., 2013; Sastre-Garriga et al., 2010). These studies did not report any significant correlations between increases in brain activity and PASAT performance, but both reported positive correlations between increases in brain activity and attention tasks, such as the Trail Making Test (part A) and the Stroop task. Therefore, overactivity of the cerebellum and parietal lobe seems to reflect an adaptive neural plasticity that indicates a beneficial effect of the cognitive rehabilitation. By contrast, Bonzano et al. (2018) used a specific CR in WMF and assessed its effect on brain activation using the Paced Visual Serial Addition task (PVSAT) in MS patients with cognitive impairment in at least one cognitive domain. They found decreased brain activity in frontoparietal regions and cingulate cortex, and they found a significant positive correlation between activation of the inferior parietal lobe (IPL) and PVSAT scores both before and after the CR, indicating that patients with better performance had higher activation of this brain region.

In addition, the n-back is a task that is not used in clinical assessment but is frequently used in functional neuroimaging studies to identify the neural mechanisms supporting WMF (Jacola et al., 2014). In this regard, two studies that included cognitively preserved MS patients reported unclear results due to small sample sizes that led them to perform individual case analyses (Hubacher, DeLuca, et al., 2015; Hubacher, Kappos, et al., 2015). Nevertheless, results revealed a trend toward increased brain activation after CR in regions of the WMF network, i.e., frontoparietal regions. These increases in brain activity correlated positively with patients' performance on WMF tests, such as the SDMT. Similar to what was observed on the PASAT, the positive correlations seem to indicate a beneficial effect of CR by stimulating adaptive neuroplasticity processes. Additionally, Campbell et al. 2016 studied the effect of home-based computer-assisted CR in a sample of cognitively impaired MS patients. The results of this study revealed higher activation of right temporoparietal regions. However, no significant correlations with performance were reported, which could make it difficult to clearly interpret the meaning of these changes in brain activation.

Lastly, in the context of the first category, the Stroop task assesses attentional abilities that are not influenced by WMF. The study by Filippi et al. 2012 reported higher activation of the posterior cingulate cortex, precuneus and dorsolateral prefrontal cortex after a CR program. However, this 
study did not include correlations with performance, and so the interpretation of these increases in brain activation is unclear.

Furthermore, regarding the second category, there are several studies that assess the effect of CR in MS patients with cognitive impairment in new learning and memory abilities (Chiaravalloti et al., 2012; Dobryakova et al., 2014; Huiskamp et al., 2016) and autobiographical memories (Ernst et al., 2012; Ernst, Sourty, Roquet, Noblet, Gounot, Blanc, de Seze, et al., 2016). Unlike previous works, the studies that focused on learning and memory functions used specific CR programs in the cognitive domains assessed in the fMRI sessions. Thus, the CR program of the studies targeting new learning and memory abilities was based on the Modified Story Memory Technique, and these studies consistently reported increased activation of several frontoparietal regions, occipital regions, medial temporal lobe, and cerebellum. Only one study reported a positive correlation between the activation of the right middle frontal gyrus and performance on the CVLT scores (Chiaravalloti et al., 2012), which seems to indicate a beneficial effect of the CR. Nevertheless, the interpretation of these increases in brain activation remains unclear. Studies targeting autobiographical memories used a CR based on Mental Visual Imagery training. They reported both increased and decreased activation after the cognitive training. Increased activation was found in cuneus, precuneus, occipital regions, superior and medial temporal lobe, posterior cingulate cortex, medial frontal cortex, thalamus, and cerebellum, whereas decreased activations was found in the superior and inferior frontal cortex, anterior cingulate cortex, insula, basal ganglia, fusiform and cerebellum. However, these studies did not include correlation analyses between changes in brain activation and performance. Again, as in previously described studies, the interpretation of these changes is open to discussion.

In summary, the results so far suggest that CR programs are associated with increased brain activation. Moreover, despite the lack of correlations between the increased brain activation and task performance, but considering that these studies reported an improvement in the performance on tasks included in the CR program, these changes in brain activation would be interpreted as adaptive neural plasticity processes that indicate a beneficial effect of the cognitive rehabilitation.

\section{Functional connectivity changes after cognitive rehabilitation}

Functional connectivity (FC) reflects patterns of temporal synchronization between different brain regions (Friston, 1994). This perspective is especially relevant because MS is considered a disconnection syndrome (P. Calabrese \& Penner, 2007; Rocca et al., 2015). To investigate the effect of CR on FC, two methodological perspectives are used: a) Task-based FC, which reflects the brain functioning during the execution of a task, normally associated with a specific cognitive domain; and 
b) the resting-state FC (rsFC) perspective, where spontaneous relationships among spatially distributed brain regions are measured, usually organized in functional networks. Studies carried out so far have used the rsFC perspective (see Table 5).

Based on the categories explained in the previous section, in the first category, which includes studies that use a CR focus on attention, WMF and IPS, we found four studies.

First, the study by Pareto et al. (2018) assessed changes in brain FC using an Independent Components Analysis (ICA) following completion of the CR in the MS patients included in the study by SastreGarriga et al. (2010). ICA is a computational method for separating a multivariate signal into additive subcomponents. In the context of fMRI analyses, ICA is applied to reveal spatially independent brain networks. The findings of this study revealed both increased and decreased synchronization in RS networks. Specifically, they showed an increased synchronization in the visual medial, cerebellar, and executive networks. The increased synchronization in the executive network correlated positively with the performance on attention tests, such as TMT, SDMT, and Digits Span, thus suggesting that the clinically observed effect might be mediated through the brain synchronization changes in these areas. However, the auditory network displayed decreased synchronization. No significant correlations were found between synchronization decreases and performance or cognitive impairment; thus, it is unclear whether these changes are compensatory or maladaptive.

Second, the work by Hubacher et al. (2015) also assessed changes in the RS network after CR using the ICA method. As mentioned above, this study included a small sample of cognitively preserved MS patients, which means that the reported results should be interpreted carefully. Regarding FC changes, the authors reported increased inter-network connectivity between the FPN and the dorsal and ventral part of the DMN. Likewise, they reported that increased FC between sub-components of the DMN was related to higher performance on the SMDT, WMF, and alertness.

Third, in the aforementioned study by Filippi et al. (2012), they also assessed changes in RS networks after CR using the ICA method. They found increased FC in the PCC and IPL of the DMN, in the ACC of the salience network, and in the DPFC of the executive network. These changes in FC seem to be related to improvements in cognitive performance, but no correlations were reported in the study. In this context, the study by Parisi et al. (2014) explored changes in FC in the same sample as in the study by Filippi et al. (2012), but from a seed-to-voxel perspective. Specifically, they focused on FC changes between the ACC and the rest of the brain. From an intra-group perspective, i.e., MS trained group pre-training versus post-training sessions, the ACC showed stronger connectivity with the middle frontal gyrus and the IPL after CR. From an interaction perspective, i.e., group (HC and 
MS) x time (pre and post), the MS trained group showed increased connectivity between the ACC and the IPL after the CR, whereas they showed decreased FC between the ACC and the inferior temporal gyrus after the CR. The increased FC in the IPL obtained with intra-group and interaction analyses showed a significant positive correlation with PASAT performance, which corroborates the hypothesis proposed in the previous study by Filippi et al. (2012) that the increases in FC were related to improvements in cognitive performance.

Lastly, the study by De Giglio et al. (2016) used a seed-to-voxel analysis to explore the changes in FC after a CR program based on memory, visual attention, visual-spatial processing, and calculation. Specifically, they study the changes in FC between the thalamus and the rest of the brain. They report FC changes showing both increases and decreases in the MS trained group, compared to the MS untrained group, after the CR program. They reported increased FC between the thalamus and PCC, precuneus, and parietal lobe, whereas they found decreased FC between the thalamus and cerebellum and DPFC. The thalamic FC changes at follow-up significantly correlated with changes in the clinical scores in the MS trained group. In particular, they observed a positive correlation between SDMT and Stroop scores and increased lateral FC in the parietal cortex, as well as a negative correlation between PASAT scores and decreased FC in the cerebellum. These results suggest that CR produces varied changes in FC that seem to be related to improvements in cognitive performance. That is, these changes in FC seem to be associated with adaptive neural plasticity processes.

Within the second category, which includes studies that use a CR focus on learning and memory functions, there are two studies.

On the one hand, regarding new learning and memory abilities, Leavitt et al. (2014) used a seed-tovoxel analysis to study the FC changes associated with the sample and CR program previously reported in the Chiaravalloti et al. (2012) and Dobryakova et al. (2014) studies. They used the left and right hippocampus and the PCC as seeds to explore the FC changes between these seeds and the rest of the brain. They found an increased FC after CR between the hippocampus and the insula, the parahippocampus, the precentral gyrus, the postcentral gyrus, and the PCC. They also reported an increased FC after CR between the PCC and the thalamus, the cerebellum, and the inferior frontal gyrus. These results suggest that the CR program was powerful enough to impact the neural networks underlying memory. Furthermore, they hypothesized that the increases in FC may serve as a precursor to improved cognitive performance. Because they did not report correlations between FC and performance, this hypothesis needs to be tested in further studies. 
On the other hand, the aforementioned study by Ernst et al. (2016) also explored the FC changes associated with the CR program focusing on autobiographical memory using the ICA method. They found a decreased FC in the CC and precuneus of the posterior DMN at follow-up in MS trained patients. These FC changes were accompanied by clinical benefits. However, authors did not report correlations between FC changes and cognitive performance. 
Table 4. Brain activity changes after cognitive rehabilitation

\begin{tabular}{|c|c|c|c|c|c|c|c|c|}
\hline Study & $\mathbf{N}$ & Duration & $\begin{array}{l}\text { Cognitive rehabilitation } \\
\text { Cognitive functions trained }\end{array}$ & $\begin{array}{c}\text { fMRI } \\
\text { cognitive } \\
\text { assesment }\end{array}$ & Task/RS & $\begin{array}{l}\text { Statistical analysis } \\
\text { Contrast }\end{array}$ & Results & $\begin{array}{c}\text { Correlations with } \\
\text { clinical and } \\
\text { behavioral measures }\end{array}$ \\
\hline \multirow[t]{2}{*}{$\begin{array}{l}\text { Sastre- } \\
\text { Garriga } \\
(2010)\end{array}$} & \multirow[t]{2}{*}{15} & \multirow{2}{*}{$\begin{array}{l}5 \text { weeks } \\
3 \text { session/week } \\
60 \mathrm{~min} / \mathrm{session} \\
\text { Total }=15 \mathrm{~h}\end{array}$} & $\begin{array}{l}\text { Computer-aided and no- } \\
\text { computer-aided games-like } \\
\text { group }\end{array}$ & \multirow[t]{2}{*}{ PASAT } & \multirow[t]{2}{*}{ Task } & $\begin{array}{l}\text { Paired t-test } \\
\text { POST > PRE }\end{array}$ & \multirow{2}{*}{$\begin{array}{l}\uparrow \mathrm{R} \text { posterior lobe of } \\
\text { cerebellum (declive and } \\
\text { uvula) } \\
\uparrow \mathrm{L} \text { anterior and posterior } \\
\text { lobes of the cerebellum } \\
\text { (declive and culmen) }\end{array}$} & \multirow[t]{2}{*}{$\begin{array}{c}\uparrow \mathrm{R} \text { culmen }-\uparrow \\
\text { TMTA }\end{array}$} \\
\hline & & & $\begin{array}{l}\text { Multi-domain: attention, } \\
\text { IPS, short-term memory, } \\
\text { WMF, and executive } \\
\text { functions. }\end{array}$ & & & & & \\
\hline \multirow{3}{*}{$\begin{array}{l}\text { Chiaravalloti } \\
\text { et al (2012) }\end{array}$} & \multirow{3}{*}{$\begin{array}{c}16 \\
M S u=8 \\
M S t=8\end{array}$} & \multirow{3}{*}{$\begin{array}{l}5 \text { weeks } \\
2 \text { session/week } \\
45-60 \\
\mathrm{~min} / \text { session }\end{array}$} & \multirow{3}{*}{$\begin{array}{l}\text { Story Memory Technique } \\
\text { New learning and memory }\end{array}$} & \multirow{3}{*}{$\begin{array}{l}\text { List-learning } \\
\text { task and word- } \\
\text { recognition } \\
\text { task }\end{array}$} & \multirow{3}{*}{ Task } & $\begin{array}{l}2 \times 2 \text { mixed ANOVA } \\
\text { Between-subject factor } \\
\text { Group }(M S u \text { vs } M S t) \\
\text { Within-subject factor } \\
\text { Time (PRE vs POST) }\end{array}$ & & \multirow{3}{*}{$\begin{array}{c}\uparrow \mathrm{R} \text { middle frontal } \\
\text { gyrus }-\uparrow \text { total } \\
\text { responses CVLT } \\
\text { short-delay free } \\
\text { recall }\end{array}$} \\
\hline & & & & & & $\begin{array}{c}M S t(P O S T)>M S t \\
(P R E)\end{array}$ & $\begin{array}{l}\uparrow \text { B middle frontal gyrus, R } \\
\text { inferior frontal gyrus, B } \\
\text { precuneus, R IPL, B PCC, } \\
\text { B middle temporal gyrus, } \\
\text { R cerebellar tonsil }\end{array}$ & \\
\hline & & & & & & $\begin{array}{l}\text { Group } x \text { Time } \\
\text { interaction }\end{array}$ & $\begin{array}{l}\uparrow \mathrm{L} \text { middle frontal gyrus, } \mathrm{R} \\
\text { precentral gyrus, } \mathrm{L} \text { inferior } \\
\text { frontal gyrus, } \mathrm{R} \text { superior } \\
\text { parietal lobule, B } \\
\text { precuneus, R PCC, L } \\
\text { supramarginal gyrus, R } \\
\text { hypothalamus, B superior } \\
\text { temporal gyrus, B CC, R } \\
\text { parahippocampal gyrus, L } \\
\text { cerebellar tonsil, R } \\
\text { culmen, R pyramis of } \\
\text { vermis, L inferior occipital } \\
\text { gyrus }\end{array}$ & \\
\hline
\end{tabular}




\begin{tabular}{|c|c|c|c|c|c|c|c|c|}
\hline \multirow[t]{2}{*}{$\begin{array}{l}\text { Ernst et al } \\
(2012)\end{array}$} & \multirow{2}{*}{$\begin{aligned} M S u & =4 \\
M S t & =4\end{aligned}$} & $\begin{array}{l}6 \text { weeks } \\
1 \text { session/week } \\
120 \\
\text { min/session }\end{array}$ & \multirow[t]{2}{*}{$\begin{array}{l}\text { Autobiographical Memory } \\
\text { Facilitation Programme } \\
\text { Autobiographical memory }\end{array}$} & \multirow{2}{*}{$\begin{array}{l}\text { Experimental } \\
\text { task (evocation } \\
\text { of specific } \\
\text { personal } \\
\text { memories) vs } \\
\text { Control task } \\
\text { (sentence } \\
\text { construction) }\end{array}$} & \multirow[t]{2}{*}{ Task } & $\begin{array}{c}\text { Paired t-test } \\
M S t(P O S T)>M S t \\
(P R E)\end{array}$ & $\begin{array}{l}\uparrow \mathrm{R} \text { cuneus, } \mathrm{L} \\
\text { inferior/middle occipital } \\
\text { gyrus, L precuneus, L } \\
\text { MTL, B PCC, R middle } \\
\text { superior temporal gyrus, R } \\
\text { superior frontal gyrus, B } \\
\text { thalamus, B cerebellum }\end{array}$ & \multirow[t]{2}{*}{ No reported } \\
\hline & & Total $\approx 12 \mathrm{~h}$ & & & & $\begin{array}{c}\text { Paired t-test } \\
M S t(P O S T)<M S t \\
(P R E)\end{array}$ & $\begin{array}{l}\downarrow \text { B superior frontal gyrus, } \mathrm{R} \\
\text { inferior frontal gyrus, } \mathrm{R} \\
\text { ACC, } \mathrm{R} \text { insula, R putamen, } \\
\text { R caudate }\end{array}$ & \\
\hline \multirow{4}{*}{$\begin{array}{l}\text { Filippi et al } \\
\text { (2012) }\end{array}$} & \multirow{4}{*}{$\begin{array}{c}20 \\
M S u= \\
10 \\
M S t=10\end{array}$} & \multirow{4}{*}{$\begin{array}{l}12 \text { weeks } \\
3 \text { session/week } \\
60 \mathrm{~min} / \mathrm{session}\end{array}$} & \multirow{4}{*}{$\begin{array}{l}\text { RehaCom package: } \\
\text { Computer-assisted } \\
\text { cognitive rehabilitation. } \\
\text { Attention, IPS and executive } \\
\text { functions }\end{array}$} & \multirow{4}{*}{$\begin{array}{l}\text { Stroop task } \\
\text { (attention and } \\
\text { executive } \\
\text { functions) }\end{array}$} & \multirow{4}{*}{$\begin{array}{l}\text { Task and } \\
\text { RS* }\end{array}$} & $\begin{array}{c}\text { Paired t-test } \\
M S t(P O S T)>M S t \\
(P R E)\end{array}$ & $\begin{array}{l}\uparrow \text { B PCC, B precuneus, B } \\
\text { DPFC }\end{array}$ & \multirow{4}{*}{$\begin{array}{c}\text { Not specific } \\
\text { correlations reported }\end{array}$} \\
\hline & & & & & & $\begin{array}{c}\frac{\text { Two-sample t-test }}{\underline{(\mathrm{POST})}} \\
M S t>M S u\end{array}$ & $\uparrow \mathrm{B}$ DPFC & \\
\hline & & & & & & $\begin{array}{l}2 \times 2 \text { mixed ANOVA } \\
\text { Between-subject factor } \\
\text { Group }(M S u \text { vs } M S t) \\
\text { Within-subject factor } \\
\text { Time (PRE vs POST) }\end{array}$ & \multirow[t]{2}{*}{$\begin{array}{l}\uparrow P C C / \text { precuneus and L } \\
\text { DPFC }\end{array}$} & \\
\hline & & & & & & $\begin{array}{l}\text { Group x Time } \\
\text { interaction }\end{array}$ & & \\
\hline
\end{tabular}




\begin{tabular}{|c|c|c|c|c|c|c|c|c|}
\hline \multirow{4}{*}{$\begin{array}{l}\text { Cerasa et al } \\
\text { (2013) }\end{array}$} & & & \multirow{4}{*}{$\begin{array}{l}\text { RehaCom package: } \\
\text { Computer-assisted } \\
\text { cognitive rehabilitation. } \\
\text { Attention and IPS }\end{array}$} & \multirow{4}{*}{ PASAT } & \multirow{4}{*}{ Task } & $\begin{array}{l}2 \times 2 \text { mixed ANOVA } \\
\text { Between-subject factor } \\
\text { Group }(M S u \text { vs } M S t) \\
\text { Within-subject factor } \\
\text { Time (PRE vs POST) }\end{array}$ & & \\
\hline & $\begin{array}{c}23 \\
M S u= \\
12 \\
M S t=11\end{array}$ & $\begin{array}{l}6 \text { weeks } \\
2 \text { session/week } \\
60 \mathrm{~min} / \mathrm{session} \\
\text { Total } \approx 12 \mathrm{~h}\end{array}$ & & & & $\begin{array}{l}\text { ROI Analysis } \\
\text { ROIs = ACC, lateral } \\
\text { premotor cortex, DPFC, } \\
\text { VPFC, superior parietal, } \\
\text { IPS, superior and middle } \\
\text { temporal gyrus, } \\
\text { thalamus, caudate and } \\
\text { cerebellum }\end{array}$ & $\begin{array}{l}\uparrow \mathrm{R} \text { posterior cerebellar } \\
\text { lobule and } \mathrm{L} \text { superior } \\
\text { parietal lobule }\end{array}$ & \multirow[t]{2}{*}{$\begin{array}{c}\uparrow \mathrm{R} \text { posterior } \\
\text { cerebellar lobule } \\
\text { and } \uparrow \mathrm{L} \text { superior } \\
\text { parietal lobule - } \uparrow \\
\text { Stroop Test }\end{array}$} \\
\hline & & & & & & $\begin{array}{l}\text { Group } x \text { Time } \\
\text { interaction }\end{array}$ & & \\
\hline & & & & & & $\begin{array}{l}\text { Whole brain analysis } \\
\text { Group } x \text { Time } \\
\text { interaction }\end{array}$ & No changes & $\begin{array}{c}\uparrow \text { DPFC }-\uparrow \text { Stroop } \\
\text { Test }\end{array}$ \\
\hline \multirow{3}{*}{$\begin{array}{l}\text { Dobryakova } \\
\text { et al (2014) }\end{array}$} & \multirow{3}{*}{$\begin{array}{c}8 \\
M S u=4 \\
M S t=4\end{array}$} & \multirow{3}{*}{$\begin{array}{l}5 \text { weeks } \\
2 \text { session/week } \\
45-60 \\
\min / \text { session } \\
\text { Total } \approx 10 \mathrm{~h}\end{array}$} & \multirow{3}{*}{$\begin{array}{l}\text { Story Memory Technique } \\
\text { New learning and memory }\end{array}$} & \multirow{3}{*}{$\begin{array}{l}\text { Word } \\
\text { encoding task } \\
(6 \text { months after } \\
\text { training) }\end{array}$} & \multirow{3}{*}{ Task } & $\begin{array}{l}\underline{2 \times 3 \text { mixed ANOVA }} \\
\text { Between-subject factor } \\
\text { Group }(M S u \text { vs } M S t) \\
\text { Within-subject factor } \\
\text { Time (PRE vs FU) }\end{array}$ & & \multirow{3}{*}{ Not reported } \\
\hline & & & & & & $\begin{array}{c}\text { ROI analysis } \\
R O I s=\text { regions of } \\
\text { group } x \text { time interaction } \\
\text { in Chiaravalloti et al } \\
\text { (2012). } \\
\text { Group } x \text { Time } \\
\text { interaction }\end{array}$ & $\begin{array}{l}\text { L middle frontal gyrus, } \mathrm{L} \\
\text { IPL, L middle occipital } \\
\text { gyrus and R cerebellar } \\
\text { tonsils }\end{array}$ & \\
\hline & & & & & & $\begin{array}{c}\text { Whole brain analysis } \\
\begin{array}{c}\text { Group } x \text { Time } \\
\text { interaction }\end{array}\end{array}$ & $\begin{array}{c}\text { R lingual gyrus, } B \\
\text { MTL/insula, and the R IPL }\end{array}$ & \\
\hline
\end{tabular}




\begin{tabular}{|c|c|c|c|c|c|c|c|c|}
\hline $\begin{array}{l}\text { Hubacher et } \\
\text { al (2015) }\end{array}$ & $5 M S t$ & $\begin{array}{l}4 \text { weeks } \\
4 \text { session/week } \\
45 \mathrm{~min} / \mathrm{session}\end{array}$ & $\begin{array}{l}\text { BrainStim: computerized } \\
\text { cognitive training }\end{array}$ & N-back & $\begin{array}{l}\text { Task and } \\
\text { RS* }\end{array}$ & $\frac{\text { Individual case }}{P O S T>P R E}$ & $\uparrow$ Regions of WMF network & $\begin{array}{c}\uparrow \text { Regions of WMF } \\
\text { network - } \uparrow \text { SMDT } \\
\text { and WMF test and } \\
\text { alertness scores }\end{array}$ \\
\hline $\begin{array}{l}\text { Hubacher et } \\
\text { al (2015b) }\end{array}$ & $10 M S t$ & Total $\approx 12 \mathrm{~h}$ & $\begin{array}{l}\text { Verbal and visual-spatial } \\
\text { aspects of } W M F\end{array}$ & & Task & $\frac{\text { Individual case }}{P O S T>P R E}$ & $\begin{array}{l}\sim \text { Distinct individual changes } \\
\text { regarding activation } \\
\text { patterns after training }\end{array}$ & Not reported \\
\hline $\begin{array}{l}\text { Campbell et } \\
\text { al (2016) }\end{array}$ & $\begin{array}{c}35 \\
M S u= \\
17 \\
M S t=18\end{array}$ & $\begin{array}{l}6 \text { weeks } \\
3 \text { session/week } \\
45 \mathrm{~min} / \text { session } \\
\text { Total } \approx 14 \mathrm{~h}\end{array}$ & $\begin{array}{l}\text { RehaCom package: } \\
\text { Computer-assisted } \\
\text { cognitive rehabilitation. } \\
\text { WMF, visuospatial memory, } \\
\text { and divided attention }\end{array}$ & N-back & Task & $\begin{array}{l}\underline{3 \times 2 \text { mixed ANOVA }} \\
\text { Between-subject factor } \\
\text { Group }(M S u \text { vs } M S t) \\
\text { Within-subject factor } \\
\text { Time }(P R E, P O S T, F U) \\
\text { Group } x \text { Time } \\
\text { interaction }\end{array}$ & $\begin{array}{l}\uparrow \mathrm{R} \text { temporoparietal regions } \\
\text { (supramarginal and } \\
\text { angular gyri) }\end{array}$ & Not reported \\
\hline $\begin{array}{l}\text { Ernst et al } \\
(2016)\end{array}$ & $\begin{array}{c}20 \\
M S u=10 \\
M S t= \\
10\end{array}$ & $\begin{array}{l}3-6 \text { weeks } \\
1-2 \\
\text { session/week } \\
120 \\
\text { min/session } \\
6 \text { sessions } \\
\text { Total } \approx 12 \mathrm{~h}\end{array}$ & $\begin{array}{l}\text { MVI programme (mental } \\
\text { visualisation exercises of } \\
\text { increasing difficulty) }\end{array}$ & $\begin{array}{l}\text { Task-related } \\
\text { (AM evocation } \\
\text { and } \\
\text { construction) } \\
\text { Control task } \\
\text { (sentence } \\
\text { construction) }\end{array}$ & $\begin{array}{l}\text { Task an } \\
\text { RS* }\end{array}$ & $\begin{array}{l}\text { Paired t-test } \\
P O S T<P R E\end{array}$ & $\begin{array}{l}\frac{\text { AM construction }}{\uparrow} \\
\begin{array}{l}\mathrm{L} \text { medial frontal regions } \\
\text { and } \mathrm{R} \text { thalamus }\end{array} \\
\begin{array}{l}\downarrow \mathrm{AM} \text { elaboration } \\
\text { frontal gyrus, } \mathrm{L} \text { fusiform } \\
\text { and } \mathrm{L} \text { cerebellum }\end{array}\end{array}$ & Not reported \\
\hline $\begin{array}{l}\text { Huiskamp et } \\
\text { al (2016) }\end{array}$ & $\begin{array}{c}16 \\
M S u=7 \\
M S t=9\end{array}$ & $\begin{array}{l}5 \text { weeks } \\
2 \text { session/week } \\
45-60 \\
\mathrm{~min} / \mathrm{session} \\
\text { Total } \approx 10 \mathrm{~h}\end{array}$ & $\begin{array}{l}\text { Modified Story Memory } \\
\text { Technique } \\
\text { Imagery and context to } \\
\text { facilitate learning }\end{array}$ & N-back & Task & $\begin{array}{l}2 \times 2 \times 3 \text { mixed ANOVA } \\
\text { Between-subject factor } \\
\text { Group }(M S u \text { vs } M S t) \\
\text { Within-subject factors } \\
\text { Time }(P R E, P O S T) \\
\text { Task }(0,1 \text { and } 2 \text {-back }) \\
\\
\text { ROI analysis } \\
\text { ROIs = superior frontal } \\
\text { gyrus, PCC, DLPFC, } \\
\text { culmen, IPL, SMA, } \\
\text { ACC }\end{array}$ & $\begin{array}{l}\text { During 1-back } \\
\uparrow \text { IPL and SMA } \\
\text { During 2-back } \\
\uparrow \text { IPL and DLPFC }\end{array}$ & No reported \\
\hline
\end{tabular}




\begin{tabular}{|c|c|c|c|c|c|c|c|c|}
\hline $\begin{array}{l}\text { Bonzano et } \\
\text { al (2018) }\end{array}$ & 18 & $\begin{array}{l}8 \text { weeks } \\
5 \text { session/week } \\
30 \mathrm{~min} / \text { session } \\
\text { Total } \approx 20 \mathrm{~h}\end{array}$ & $\begin{array}{l}\text { COGNI-TRAcK: } \\
\text { Adaptive computer-assisted } \\
\text { cognitive rehabilitation. } \\
\text { And non-adaptive training } \\
\text { on WMF (visuospatial task, } \\
\text { operation N-back task and a } \\
\text { dual N-back task }\end{array}$ & PVSAT & Task & $\frac{\text { Paired t-test }}{P O S T<P R E}$ & $\begin{array}{l}\downarrow \text { L CC, R postcentral gyrus } \\
\text { and R IPL }\end{array}$ & $\uparrow \mathrm{R}$ IPL $-\uparrow \mathrm{PVSAT}$ \\
\hline
\end{tabular}

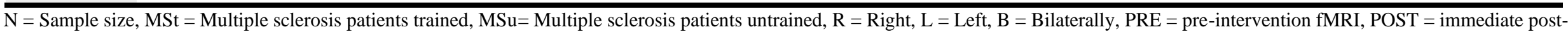

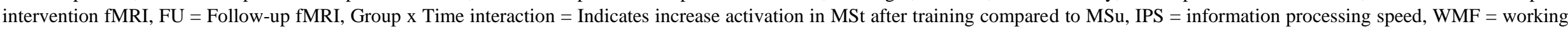

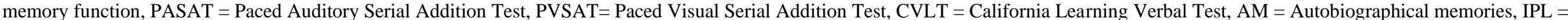

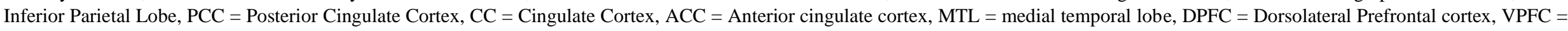
Ventral prefrontal cortex, SMA = Supplementary Motor Area, RS*= Results in table 3 
Table 5. Functional connectivity changes after cognitive rehabilitation

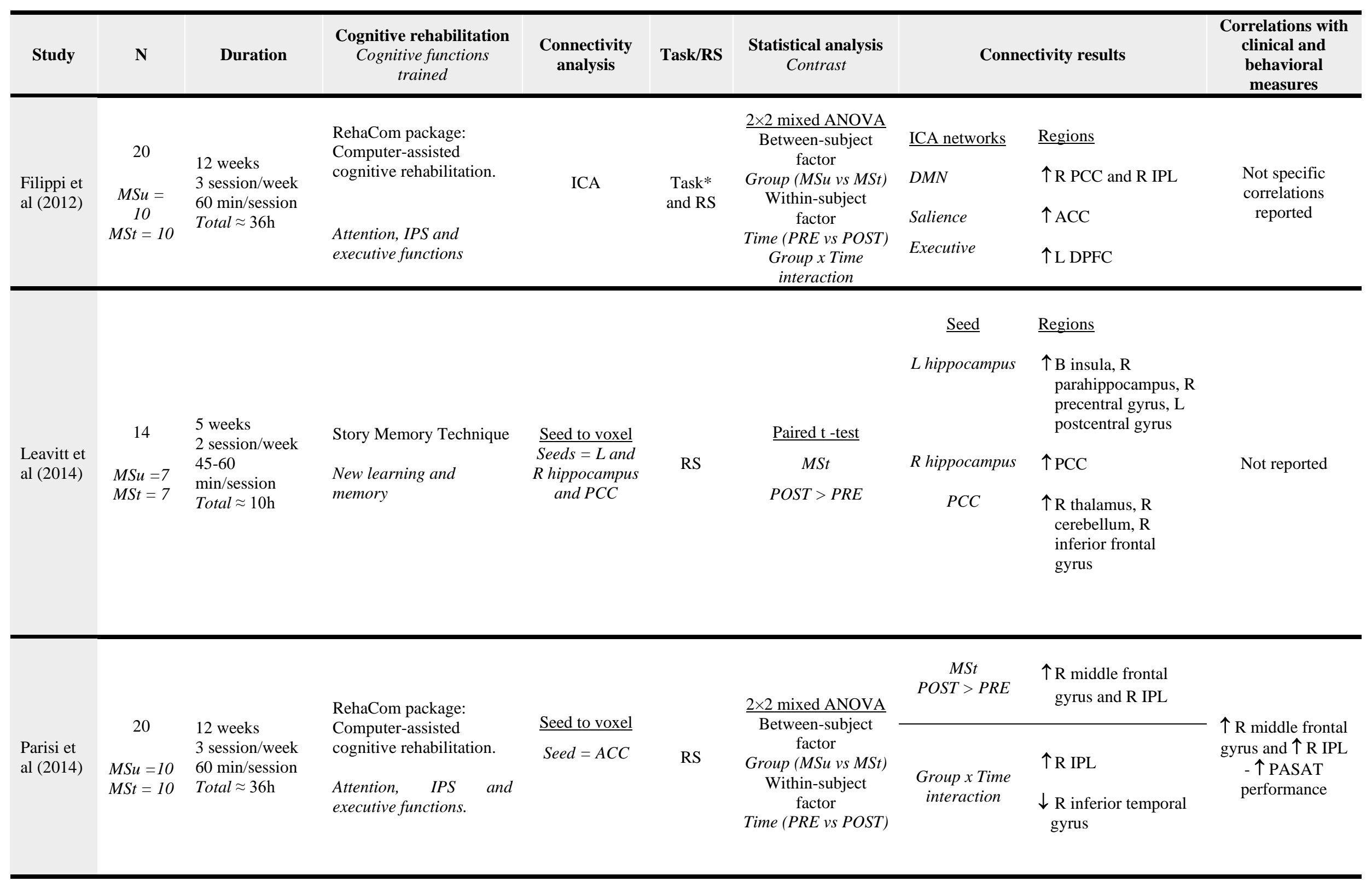




\begin{tabular}{|c|c|c|c|c|c|c|c|c|c|}
\hline $\begin{array}{l}\text { Bonavita et al } \\
\text { (2015) }\end{array}$ & $\begin{array}{c}20 \\
\\
M S u \\
=14 \\
M S t= \\
18\end{array}$ & $\begin{array}{l}8 \text { weeks } \\
2 \text { session/week } \\
50 \mathrm{~min} / \mathrm{session} \\
\text { Total } \approx 14 \mathrm{~h}\end{array}$ & $\begin{array}{l}\text { RehaCom package: } \\
\text { Computer-assisted } \\
\text { cognitive rehabilitation. } \\
\begin{array}{l}\text { Attention, IPS and } \\
\text { executive functions. }\end{array}\end{array}$ & $\frac{\underline{\mathrm{ICA}}}{D M N}$ & $\mathrm{RS}$ & $\begin{array}{c}\frac{\text { Multi-subject }}{\text { random effects 2- }} \\
\frac{\text { way ANOVA }}{\text { Random }} \\
\text { observations } \\
\text { Individual subject } \\
\text { map values as at } \\
\text { each voxel and } \\
\text { cluster } \\
\text { Within-subject } \\
\text { factor } \\
\text { Time (PRE, POST) }\end{array}$ & $\begin{array}{c}M S t \\
P O S T>P R E\end{array}$ & $\uparrow P C C$ and IPL & $\begin{array}{c}\text { PCC - } \downarrow \text { Stroop } \\
\text { Test }\end{array}$ \\
\hline $\begin{array}{l}\text { Hubacher et al } \\
\text { (2015) }\end{array}$ & $5 \mathrm{MSt}$ & $\begin{array}{l}4 \text { weeks } \\
4 \text { session/week } \\
45 \mathrm{~min} / \text { session } \\
\text { Total } \approx 12 \mathrm{~h}\end{array}$ & $\begin{array}{l}\text { BrainStim: } \\
\text { Computer-assisted } \\
\text { cognitive rehabilitation. } \\
\text { Verbal and visual-spatial } \\
\text { aspects of WMF }\end{array}$ & ICA & $\begin{array}{c}\text { Task* } \\
\text { and RS }\end{array}$ & Individual case & $\begin{array}{l}\text { Temporal } \\
\text { correlations } \\
\text { between } \\
\text { RSNs }\end{array}$ & $\begin{array}{l}\uparrow \text { Increased } \\
\text { connectivity } \\
\text { between R FPN } \\
\text { and dorsal or } \\
\text { ventral DMN }\end{array}$ & $\begin{array}{c}\text { DMN } \\
\text { subcomponents - } \\
\uparrow \text { SMDT, WMF } \\
\text { test and alertness } \\
\text { scores }\end{array}$ \\
\hline \multirow{2}{*}{ DeGiglio (2016) } & 22 & \multirow{3}{*}{$\begin{array}{l}8 \text { weeks } \\
5 \text { session/week } \\
30 \mathrm{~min} / \text { session }\end{array}$} & \multirow{3}{*}{$\begin{array}{l}\text { Video-game training } \\
\text { program: } \\
\text { Memory, attention, } \\
\text { visual-spatial } \\
\text { processing, } \\
\text { and calculation. }\end{array}$} & \multirow{2}{*}{$\begin{array}{l}\text { eed to voxel } \\
\text { Seed }= \\
\text { thalamus }\end{array}$} & \multirow{2}{*}{$\mathrm{RS}$} & \multirow{2}{*}{$\begin{array}{c}\text { Two Sample t-test } \\
\text { POST }\end{array}$} & $\mathrm{MSt}>\mathrm{MSu}$ & $\begin{array}{l}\uparrow \text { PCC, precuneus, B } \\
\text { parietal cortex }\end{array}$ & \multirow{2}{*}{$\begin{array}{c}\uparrow \mathrm{B} \text { lateral } \\
\text { parietal cortex - } \\
\uparrow \text { SDMT } \\
\uparrow \mathrm{R} \text { lateral } \\
\text { parietal cortex - } \\
\uparrow \text { Stroop Test } \\
\uparrow \mathrm{R} \text { cerebellum - } \\
\downarrow \text { PASAT }\end{array}$} \\
\hline & $\begin{array}{c}=11 \\
M S t= \\
11\end{array}$ & & & & & & $\mathrm{MSu}>\mathrm{MSt}$ & $\begin{array}{l}\downarrow \text { Vermis, cerebellar } \\
\text { hemispheres and L } \\
\text { DPFC }\end{array}$ & \\
\hline $\begin{array}{l}\text { DeGiglio et al } \\
(2016)\end{array}$ & 18 & & & $\begin{array}{l}\text { DTI of corpus } \\
\text { callosum }\end{array}$ & Structural & $\begin{array}{l}\text { FA } \\
\text { Mean, axial and } \\
\text { radial diffusivity }\end{array}$ & $\begin{array}{c}M S t \\
P O S T>P R E\end{array}$ & $\begin{array}{l}\downarrow \text { Lower axial } \\
\text { diffusivity in the } \\
\text { corpus callosum }\end{array}$ & $\begin{array}{l}\uparrow \text { corpus } \\
\text { callosum - } \downarrow \\
\text { PASAT }\end{array}$ \\
\hline Ernst et al (2016) & $\begin{array}{c}20 \\
M S u \\
=10 \\
M S t= \\
10\end{array}$ & $\begin{array}{l}3-6 \text { weeks } \\
1-2 \\
\text { session/week } \\
120 \\
\mathrm{~min} / \text { session } \\
6 \text { sessions } \\
\text { Total } \approx 12 \mathrm{~h}\end{array}$ & $\begin{array}{l}\text { MVI programme (mental } \\
\text { visualisation exercises of } \\
\text { increasing difficulty) }\end{array}$ & ICA & $\begin{array}{l}\text { Task* } \\
\text { and RS }\end{array}$ & $\begin{array}{c}\text { Paired t-test } \\
\text { MSt (POST) }<\text { MSt } \\
(\text { PRE) }\end{array}$ & $\begin{array}{l}\text { Posterior } \\
\text { DMN }\end{array}$ & $\begin{array}{l}\downarrow \mathrm{CC} \text { and } \\
\text { precuneus }\end{array}$ & Not reported \\
\hline
\end{tabular}




\begin{tabular}{|c|c|c|c|c|c|c|c|c|c|}
\hline \multirow{5}{*}{$\begin{array}{l}\text { Pareto et al } \\
(2018)\end{array}$} & \multirow{5}{*}{15} & \multirow{5}{*}{$\begin{array}{l}5 \text { weeks } \\
4 \text { session/week } \\
60 \mathrm{~min} / \mathrm{session} \\
\text { Total } \approx 20 \mathrm{~h}\end{array}$} & \multirow{5}{*}{\multicolumn{2}{|c|}{$\begin{array}{l}\text { Computer-aided and no- } \\
\text { computer-aided games- } \\
\text { like group } \\
\text { Multi-domain: attention, synchronization } \\
\text { IPS, short-term memory, } \\
\text { WMF, and executive } \\
\text { functions }\end{array}$}} & \multirow{5}{*}{$\mathrm{RS}$} & \multirow{5}{*}{$\frac{\text { Paired-t test }}{P R E \text { vs POST }}$} & ICA networks & Regions & \multirow{5}{*}{$\begin{array}{l}\uparrow \mathrm{R} \text { frontal middle } \\
\text { orbital (Executive } \\
\text { network) - } \uparrow \\
\text { composite } \mathrm{Z} \text {-score }\end{array}$} \\
\hline & & & & & & & Visual medial & $\begin{array}{l}\uparrow \mathrm{R} \text { inferior temporal } \\
\text { and } \mathrm{L} \text { cerebral crus } \\
1, \downarrow \mathrm{L} \text { superior } \\
\text { temporal pole }\end{array}$ & \\
\hline & & & & & & & Auditory & $\begin{array}{l}\downarrow \mathrm{R} \text { middle temporal } \\
\text { pole }\end{array}$ & \\
\hline & & & & & & & Cerebellum & $\uparrow \mathrm{R}$ superior parietal & \\
\hline & & & & & & & Executive & $\begin{array}{l}\uparrow \mathrm{R} \text { frontal middle } \\
\text { orbital }\end{array}$ & \\
\hline
\end{tabular}

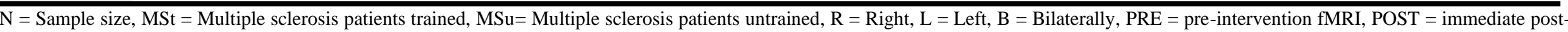

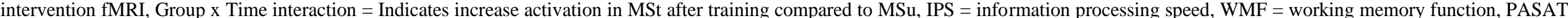

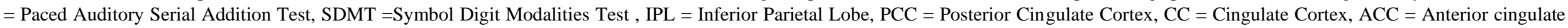
cortex, DPFC = Dorsolateral prefrontal cortex, RSNs=Resting-State Networks, DMN = Default Mode Network, FPN= Fronto Parietal Network, Task*= Results in table 2 


\section{CHAPTER 2}

\section{EXPERIMENTAL SECTION}




\section{Study I}

\section{Lesion-Functional Disconnectomics in Multiple Sclerosis}

Naiara Aguirre ${ }^{1}$, Will Orwig ${ }^{2,3}$, Álvaro-Javier Cruz-Gómez ${ }^{3,4}$, Anna Miró-Padilla ${ }^{1}$, Elisenda Bueichekú $^{2}$, César Ávila ${ }^{1}$, Carla Sanchis-Segura ${ }^{1}$, Cristina Forn ${ }^{1}$ and Jorge Sepulcre ${ }^{2,3}$

${ }^{1}$ Jaume I University. Department of Basic, Clinic and Psychobiology. Neuropsychology and Functional Neuroimaging group

${ }^{2}$ Gordon Center for Medical Imaging,Department of Radiology, MassachusettsGeneral Hospital, Harvard Medical School,Boston, Massachusetts

${ }^{3}$ Athinoula A. Martinos Center for BiomedicalImaging, Department of Radiology,Massachusetts General Hospital, HarvardMedical School, Charlestown, Massachusett

${ }^{4}$ INiBICA. Instituto de Investigación e Innovación Biomédica de Cádiz

Under review 


\begin{abstract}
Background: Multiple Sclerosis (MS) is a neurodegenerative disease primarily characterized by demyelinating lesions leading to neuro-disconnectivity which mechanism is not well understood. Characterizing how widespread lesions relate to multiple cortical areas remains a computational challenge. In this study, we present a novel approach to detect and investigate topologically specific associations between white matter lesions and changes in cortical connectivity.
\end{abstract}

Methods: Our sample includes 33 healthy controls (HC) (mean age: 32.39 $\pm 7.12,18$ males) and 29 relapsing-remitting MS patients (mean age: $35.83 \pm 6.45,9$ males) with no cognitive impairments. To identify white matter (WM) lesions, we used the lesion prediction algorithm as implemented in the LST toolbox using normalized FLAIR images. After that, we estimated the lesion volume of each JHU ICBM atlas region and we selected only those regions that at least $80 \%$ of participants showed lesion. Then, we computed the weighted degree (WD) map for each subject using resting-state functional connectivity (FC) data. Additionally, we performed a modularity graph analysis (SFC) to explore the underlying network-wise mechanisms of detected dysconnectivity changes. This method provides a modularity segregation of FC in which connections between neighboring nodes (local connectivity) and connections between distant nodes (distributed connectivity) can be investigated separately. Then, we segregated the FC maps (WD and SFC) into the seven Yeo networks and we performed a linear regression analysis to describe the relationship between WM lesion volumes and gray matter FC maps. We also used Spearman correlations to study the relationship between FC alterations. Finally, we studied significant differences between these correlation coefficients of HC group and MS patients group using the Fisher r-to-z transformation.

Results: In MS group, left posterior limb of the internal capsule lesions are consistently associated with increases in FC, concretely with local-FC. Likewise, the increases in FC were related with lower scores in neuropsychological tests that measure the most affected cognitive domains in MS.

Conclusions: The increases in FC can be interpreted as compensatory mechanisms or maladaptive processes secondary to WM-lesions. Further research with MS patients of heterogeneous phenotypes and different degree of cognitive impairment is needed in order to clarify the interpretation of the FC changes. Nevertheless, the combined use of graph theory measures and WM-lesion location seems to be a promising tool to understand the development of MS pathology and its effect on brain functionality.

Keywords: Multiple sclerosis, white matter lesions, functional connectivity, graph theory 


\section{Introduction}

The relationship between white matter (WM) lesions, brain functionality, and cognitive deficits in multiple sclerosis (MS) remains largely unknown. In the past, mismatches have been consistently reported between clinical outcomes and the prevalence of WM lesions (Fulton et al., 1999; Heesen et al., 2010; Hulst et al., 2013, 2014; Mollison et al., 2017; Uher et al., 2014); a phenomenon historically known as the clinic-radiological paradox (Barkhof, 1999, 2002). Despite the more recent characterization of diffuse microscopic WM abnormalities and cortical lesions as part the MS pathology (Calabrese et al., 2015; Lee et al., 2014), the multifaceted and topologically heterogenous nature of MS is still obfuscating the structural-functional relationships of the disease.

Over the last decade, functional connectivity (FC) MRI has provided a powerful tool to study the link between MS lesions and brain (dis)functionality. For instance, previous studies have reported regional increases of FC in clinically isolated syndrome and early MS patients (Gamboa et al., 2014; Hawellek et al., 2011; Louapre et al., 2014; Rocca et al., 2020), as well as decreased FC in secondary progressive and primary progressive MS patients related to disease progression (Rocca, Valsasina, et al., 2010). However, studies with relapsing-remitting MS (RRMS) patients yield inconsistent findings, both FC increases and decreases have been reported in similar systems (Louapre et al., 2014; Tahedl et al., 2018), in which different factors, such as disease duration, cognitive status, lesion load and location among others may play important roles (Castellazzi et al., 2018; Soares et al., 2021; Tewarie et al., 2018). An inverse U-curve effect has been suggested to explain the relationship between WM damage and FC (Fleischer et al., 2019; Tewarie et al., 2018). Initially, structural disconnection seems to be related with increased FC, associated with compensatory mechanisms (Fleischer et al., 2017; Muthuraman et al., 2016; Rocca, Absinta, et al., 2010) which reach their limits due to the built-up damage resulting in decrease in overall FC (Fleischer et al., 2019; Schoonheim, Meijer, et al., 2015; Tewarie et al., 2018). Although this interpretation fits well with heterogenous and widespread lesion burden, the precise spatial relationships between localized structural damage and FC remains largely unknown. Moreover, findings about the independent effects of GM and WM structural damage on FC are scarce. Understanding of how specific lesions of brain tissue are related with FC changes requires further research on this topic (Fleischer et al., 2019; Soares et al., 2021; Tahedl et al., 2018). Extant research has mainly focused on the relationship between functional connectivity or cognitive impairment and WM lesion burden, though less attention has been paid to spatial location perspectives (Droby et al., 2016; Fleischer et al., 2019). This may be attributed to the methodological difficulty of studying MS-lesions on multiple sites and interpretation of MS as a disconnection disease (P. Calabrese \& Penner, 2007; Tahedl et al., 2018). In the present study, we used a novel network- 
wise approach to detect and investigate the topological impact of the location of WM-lesions on functional connectivity networks in MS based on graph theory metrics (Bassett \& Sporns, 2017; Diez \& Sepulcre, 2018; Rubinov \& Sporns, 2010; Sepulcre, Sabuncu, Yeo, et al., 2012; Tahedl et al., 2018). This framework allowed us to characterize key spatial integration features of disease-related structural and functional alterations in MS.

\section{Methods}

\subsection{Participants and study design}

Our sample consisted of 33 healthy controls (age $=32.39 \pm 7.12$, men=18) and 29 patients (age= $35.83 \pm 6.45$, men=9) diagnosed with clinically definite RR MS according to McDonald's criteria (Polman et al., 2011). Exclusion criteria for patients were: appearance of new flares-ups, use of steroids and introduction or modification of any medication in the previous two months, any relevant concomitant diseases, contraindications to MRI. All participants were right-handed and presented no cognitive impairment, assessed via the BRB-N, validated for the Spanish population (Sepulcre, Vannotti, et al., 2006); the Matrix Reasoning Subtest of the Wechsler Adult Intelligence Scale (WAIS

III) to assess the intelligence quotient (IQ); the Fatigue Severity Scale (FSS); and the Beck Depression Inventory (BDI). In addition, patients were neurologically assessed using the Expanded Disability Status Scale (EDSS). Refer to Table 1 for a detailed demographic and clinical data of all participants. Finally, a structural MRI and a resting-sate fMRI were collected for each participant. The ethics committee of University Jaume I approved the research project. Written informed consent was obtained from all participants prior to participation. 
Table 1. Demographic, clinical and neuropsychological performance data of all participants Demographic and clinical data

\begin{tabular}{lccc}
\hline & $\boldsymbol{H C}(\boldsymbol{N}=\mathbf{3 3})$ & $\boldsymbol{M S}(\boldsymbol{N}=\mathbf{2 9})$ & Between-group differences \\
\hline Age & $32.39 \pm 7.12$ & $35.83 \pm 6.45$ & $\mathrm{t}_{60}=1.98, \mathrm{p}=0.052$ \\
Gender (men/women) & $18 / 15$ & $9 / 20$ & $\mathrm{X}^{2}{ }_{62}=3.47, \mathrm{p}=0.062$ \\
Educational level (1-6 levels) & $4.30 \pm 1.13$ & $3.83 \pm 1.44$ & $\mathrm{t}_{60}=1.45, \mathrm{p}=0.151$ \\
EDSS & - & $1.55 \pm 1.53$ & - \\
Mean years disease duration & - & $7.93 \pm 4.85$ & - \\
Total lesión volumen (ml) & - & $2.89 \pm 3.90$ & - \\
FSS & - & $41.65 \pm 17.13$ & - \\
BPF & $0.81 \pm 0.03$ & $0.80 \pm 0.04$ & $\mathrm{t}_{60}=1.53, \mathrm{p}=0.130$ \\
\hline
\end{tabular}

\section{Neuropsychological data}

\begin{tabular}{lccc}
\hline & HC $(\mathbf{N = 2 2})$ & $M S(N=29)$ & Between-group differences \\
\hline BRB-N & $48 \pm 7.54$ & & \\
PASAT & $62.73 \pm 8.52$ & $47.67 \pm 9.90$ & $\mathrm{t}_{49}=0.13, \mathrm{p}=0.899$ \\
SDMT & $55.72 \pm 9.94$ & $57.97 \pm 10.21$ & $\mathrm{t}_{49}=1.77, \mathrm{p}=0.083$ \\
SRT-storage & $48.86 \pm 12.65$ & $53.24 \pm 11.43$ & $\mathrm{t}_{49}=0.81, \mathrm{p}=0.420$ \\
SRT-retrieval & $10.23 \pm 1.77$ & $44.07 \pm 12.63$ & $\mathrm{t}_{49}=1.34, \mathrm{p}=0.186$ \\
SRT-delayed & $22.10 \pm 5.69$ & $10.21 \pm 2.27$ & $\mathrm{t}_{49}=0.35, \mathrm{p}=0.972$ \\
SPART-immediate & $7.73 \pm 2.41$ & $20.55 \pm 5.40$ & $\mathrm{t}_{49}=0.99, \mathrm{p}=0.329$ \\
SPART-delayed & $13.32 \pm 4.05$ & $7.10 \pm 2.13$ & $\mathrm{t}_{49}=0.98, \mathrm{p}=0.333$ \\
WLGT & $104.29 \pm 15.67$ & $12.14 \pm 4.43$ & $\mathrm{t}_{49}=0.98, \mathrm{p}=0.333$ \\
Matrix subtest (WAIS III) & $6.64 \pm 5.53$ & $109.64 \pm 11.46$ & $\mathrm{t}_{49}=1.38, \mathrm{p}=0.173$ \\
BDI & & $12.17 \pm 8.24$ & $\mathrm{t}_{49}=2.72, \mathrm{p}=0.009 *$ \\
\hline
\end{tabular}

\subsection{MRI acquisition}

MRI data were acquired on a 1.5T scanner (Siemens Symphony, Erlangen, Germany). A multi-planar T1-weighted localizer with slice orientation parallel to the subcallosal line was acquired at the beginning of each MRI examination. For all participants, MRI protocol included the following sequences: (1) high-resolution anatomical 3D MPRAGE, using a T1-weighted gradient echo pulse sequence $\left(\mathrm{TR}=2200 \mathrm{~ms}, \mathrm{TE}=3 \mathrm{~ms}\right.$, flip angle $=15^{\circ}$, matrix $=256 \times 256 \times 160$, isomorphic voxel $\left.=1 \mathrm{~mm}^{3}\right)$; (2) sagittal 3D fluid-attenuated inversion recovery (FLAIR) (TR=6000ms, TE $=354 \mathrm{~ms}$, flip angle $=180^{\circ}$, matrix $=196 \times 256 \times 160$, voxel $\left.=1.05 \times 1.05 \times 1 \mathrm{~mm}\right)$ and $(3)$ eyes-open resting state fMRI consisted on 250 volumes, acquired using a gradient-echo T2*-weighted echo-planar imaging sequence covering the entire brain $\left(\mathrm{TR}=2300 \mathrm{~ms}, \mathrm{TE}=47 \mathrm{~ms}\right.$, matrix $=64 \times 64 \times 27$, flip angle $=90^{\circ}$, isomorphic voxel $=3.5 \mathrm{~mm}^{3}$, slice gap $=4.41 \mathrm{~mm}$ ). 


\subsection{MRI analysis}

\section{Data preprocessing}

\section{White matter lesion volumes}

Firstly, we used the lesion prediction algorithm (Schmidt, 2017) as implemented in the LST toolbox version 3.0.0 (https://www.applied-statistics.de/lst.html) to obtain the probabilistic lesion map for each MS patient using FLAIR sequence. After that, we resliced the JHU ICBM white matter labels atlas to match the anatomical MRI dimensions using nearest neighbor interpolation. Finally, we estimated the lesion volume of each JHU ICBM atlas region. To perform the following analysis, we selected only those regions that at least $80 \%$ of participants showed lesion.

\section{$\underline{\text { Resting-state fMRI }}$}

FMRIB Software Library v5.0.7 (FSL) and MATLAB 2017a were used for the resting-state fMRI pre-processing. First, the anatomical T1 was processed: reorientation to right-posterior-inferior (RPI); alignment to anterior and posterior commissures; skull stripping; gray matter, white matter and cerebrospinal fluid segmentation; and computation of non-linear transformation between individual skull-stripped T1 and 2mm resolution MNI152 template images. Then functional MRI image was preprocessed: slice time correction; reorientation to RPI; realigning functional volumes within runs with a rigid body transformations (6 parameters linear transformation); computation of the transformation between individual skull-stripped $\mathrm{T} 1$ and mean functional images; intensity normalization; removal of confounding factors from the data using linear regression - including 6 motion-related covariates (rigid motion parameters and its derivatives), linear and quadratic terms, and five components each from the lateral ventricles and white matter. No global signal regression was applied due to the spurious correlations this can introduce. Transformation of resting-state data to MNI space, concatenating the transformation from functional to structural and from structural to MNI, spatial smoothing with an isotropic Gaussian kernel of 6-mm FWHM, and band-pass filtering $(0.01-0.08 \mathrm{~Hz})$ to reduce low-frequency drift and high-frequency noise were also performed. Scrubbing of time points with excess head motion eliminated all time points with a frame displacement $>0.5 \mathrm{~mm}$. Finally, for computational efficiency, data was down-sampled to $6 \mathrm{~mm}$ to perform voxel-level analyses. 


\section{Post-processing analyses}

Post-processing analyses were conducted with Matlab2018a coding (v9.4, The Mathworks, Inc., Natick, MA). A binary mask of 5,637 voxels was used that covered the entire brain, excluding the cerebellum, to compute the whole-brain connectivity matrix at the individual level. The connectivity matrix was computed using the Pearson Correlation Coefficient between each voxel pair's time course for each participant. We obtained two matrices for each participant, one containing the correlation coefficients (r-values), and the other containing the p-values. Then, a variance-stabilizing transformation (Fisher's transformation) was applied to r-values matrix. According to previous graphbased functional connectivity approaches, the functional connectivity matrices of positive correlations represent the strength of the connectivity between each pair of voxels across the brain (i.e., the degree) (Sepulcre et al., 2010). Therefore, we used the positive correlations in the following steps of the analysis. We removed all negative r-values as only positive connectivity has been proved to drive functional connectivity network topology in the human brain (Qian et al., 2018). Likewise, we include in the analysis only connections corrected by multiple comparisons using false-discoveryrate (FDR; q<0.001) (Benjamini \& Hochberg, 1995). Uncorrected connections were removed from the functional matrices, due to the fact they are likely attributable to noise.

Using the generated connectivity matrix, we applied two different graph-based functional connectivity approaches:

\section{Weighted degree}

Degree centrality is a network measure that quantifies the number of links or edges connecting a node to the rest of the network (Rubinov \& Sporns, 2010). Weighted degree (WD) is a variant of degree centrality that quantifies the connectivity strength of one node with the rest of the brain. Voxel level weighted degree was computed to evaluate the importance of each node in the $5,637 \times 5,637$ matrices. Thus, WD for each voxel is obtained by summing the weights of all the connections between in and the rest of the brain. 
$\underline{\text { Individual local/modular and distant/distributed connectivity maps }}$

This approach was used to study network path connectivity changes over time using a whole-brain voxel stepwise functional connectivity analysis (SFC) (Diez \& Sepulcre, 2018). We extract the spatial distance information from the connectivity patterns, differentiating between local connections (modular links) and distant connections (distributed links). Local connections represent discrete brain networks, associated with specific cognitive functions. Conversely, distributed connectivity represents communication between these localized brain networks.

Local and distributed connectivity maps were generated using whole-brain stepwise functional connectivity analyses (Sepulcre, Sabuncu, Yeo, et al., 2012). Additionally, WD analysis was conducted to calculate all links in the brain, then identify individual variability between WD maps for local and distributed connectivity (Diez \& Sepulcre, 2018).

To compute the voxel-wise connectivity maps, we followed the steps described below.

$$
\begin{gathered}
N S F C_{1}(i, j)=\frac{r(i, j)-\min (r)}{\max (r)-\min (r)} \\
S F C_{s}(i, j)=\sum_{k=1}^{n} N S F C_{s-1}(i, k) N S F C_{1}(k, j)[i \neq j, s>1] \\
N S F C_{s}=\frac{S F C_{s}-\min \left(S F C_{s}\right)}{\max \left(S F C_{s}\right)-\min \left(S F C_{s}\right)}
\end{gathered}
$$

Where $r$ is the corrected association connectivity matrix, $n$ is the number of voxels, and $N S F C_{S}$ is the normalized stepwise connectivity matrix for number of steps $(s)$. 
The local connectivity matrices were computed as:

$$
\begin{gathered}
N L C_{1}(i, j)=\frac{r(i, j)-\min (r)}{\max (r)-\min (r)}\left[N S F_{2}(i, j) \neq 0\right] \\
L C_{s}(i, j)=\sum_{k=1}^{n} N L C_{s-1}(i, k) N L C_{1}(k, j)[i \neq j, s>1] \\
N L C_{s}=\frac{L C_{s}-\min \left(L C_{s}\right)}{\max \left(L C_{s}\right)-\min \left(L C_{s}\right)}
\end{gathered}
$$

The distributed connectivity matrices were computed as:

$$
\begin{gathered}
N D C_{1}(i, j)=N S F C_{4}(i, j)\left[N S F C_{1}(i, j)=N S F C_{2}(i, j)=0\right] \\
D C_{s}(i, j)=\sum_{k=1}^{n} N D C_{s-1}(i, k) N S F C_{1}(k, j)[i \neq j, s>1] \\
N D C_{s}=\frac{D C_{s}-\min \left(D C_{s}\right)}{\max \left(D C_{s}\right)-\min \left(D C_{s}\right)}
\end{gathered}
$$

Final WD maps for local and distributed connectivity were computed as the sum of steps 2 to 7 .

\section{$\underline{\text { Second-level analysis }}$}

In order to study how lesion load affects functional connectivity, we segregated the brain into the seven functional Yeo-networks (Yeo et al., 2011). We compute three Spearman's correlations for each network. Firstly, to examine how lesions affect the functional connectivity we assessed WM lesion volumes of each MS patient and their grey matter WD connectivity (WD-FC) maps. Additionally, to explore whether the effect of the WM lesions on the functional connectivity is restricted to local connectivity or also affects distributed connectivity, we measured WM lesion volumes of each MS patient and their grey matter local and distant connectivity maps. For each correlation analysis, we obtained four separate matrices, one containing the positive correlation 
coefficients, other containing the negative correlation coefficients, and the two remaining matrices containing the p-values associated to each positive and negative correlation matrices. Positive correlation matrices represent those regions that display increased functional connectivity related to an increase in lesion load, while negative correlation matrices represent those regions that display decrease functional connectivity related to an increase in lesion load. Results included only those voxels corrected by multiple comparisons using false-discovery-rate (FDR; $\mathrm{q}<0.001$ ).

Then, we extracted the WD-FC and SFC normalized values by performing log transformation, for all participants (HC and MS patients) of the resulting significant clusters to study between-group differences and correlations with BRB-N scores using SPSS 25 (IBM Corp). Finally, we studied significant differences between correlation coefficients of HC group and MS patients group using the Fisher r-to-z transformation (http://vassarstats.net/rdiff.html).

\section{Results}

JHU ICBM atlas includes forty-eight WM-tracts, of which fifteen met the inclusion criteria for analyses of presence of lesion in at least $80 \%$ of MS patients. Among these tracts, only the following four WM tracts showed significant correlations with FC measures: left posterior limb of internal capsule, right posterior limb of internal capsule, left anterior limb of internal capsule and left superior fronto-occipital fasciculus.

\subsection{Spearman correlations between FC of 7 Yeo-networks and WM lesion volumes.}

\section{$\underline{\text { Default mode network (DMN) }}$}

WD-FC and local-SFC of left triangularis inferior frontal gyrus, left superior medial frontal gyrus and left middle frontal gyrus showed significant positive correlations with left posterior limb of internal capsule lesion volume.

\section{Fronto-parietal network (FPN)}

WD-FC of left middle frontal gyrus (A46 and A8vl), right parietal inferior and left precuneus showed a significant positive correlation with left posterior limb of internal capsule lesion volume.

Local-SFC of left middle frontal gyrus (A8vl), right IPL and left precuneus showed a significant positive correlation with left posterior limb of internal capsule lesion volume. Furthermore, localSFC of right inferior temporal showed a significant positive correlation with left anterior limb of internal capsule lesion volume. 


\section{$\underline{\text { Somatomotor network (SMN) }}$}

WD-FC of right postcentral gyrus and the upper limb, head and face region (ulhf) of left postcentral gyrus showed a significant positive correlation with left posterior limb of internal capsule lesion volume. Similarly, left posterior limb of internal capsule lesion volume showed a significant positive correlation with local-SFC of right postcentral gyrus, left postcentral gyrus (ulhf) and trunk region (tru) of left postcentral gyrus.

Moreover, both WD-FC and local-SFC of left postcentral gyrus (tru) showed a significant positive correlation with right posterior limb of internal capsule lesion volume.

Additionally, local-SFC of left Rolandic operculum showed a significant positive correlation with left superior fronto-occipital fasciculus.

\section{Dorsal-attention network (DAN)}

WD-FC of DAN did not show any significant correlation with WM lesion volumes. Nevertheless, the local-SFC of right middle temporal and left middle temporal showed a significant positive correlation with left posterior limb of internal capsule lesion volume.

\section{$\underline{\text { Ventral-attention network (VAN) }}$}

WD-FC and local-SFC of left postcentral gyrus/left supramarginal gyrus showed a significant positive correlation with left posterior limb of internal capsule lesion volume.

Visual network (VN)

WD-FC and local-SFC of left lingual and left calcarine showed a significant positive correlation with left posterior limb of internal capsule.

For further information, please refer to Table 2 and Figure 1. 
Figure 1. Significant correlations between WD-FC and local-SFC of gray matter clusters of the 7 Yeo-networks and WM tracts lesion volumes.
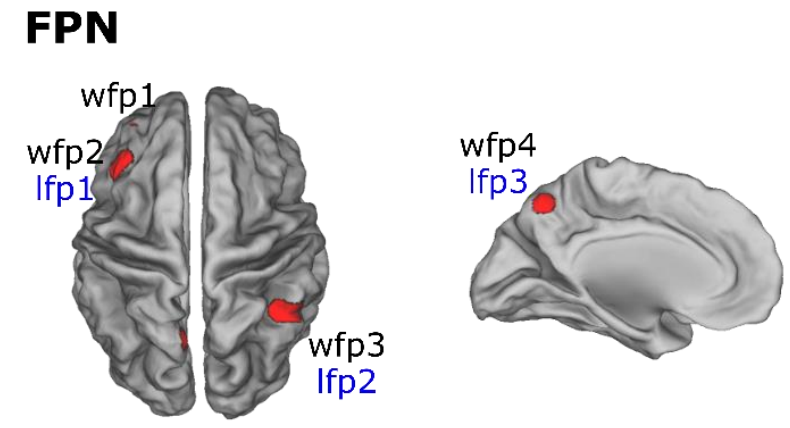

\section{SMN}
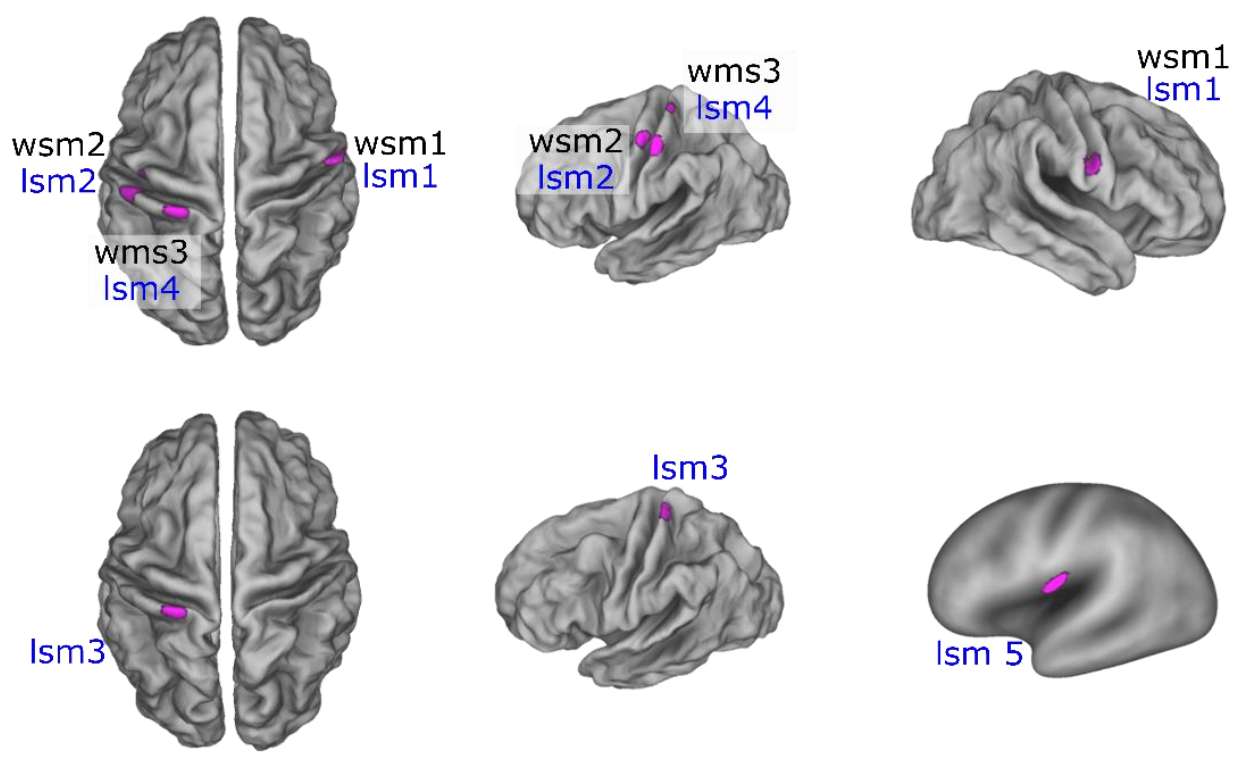

\section{DMN}
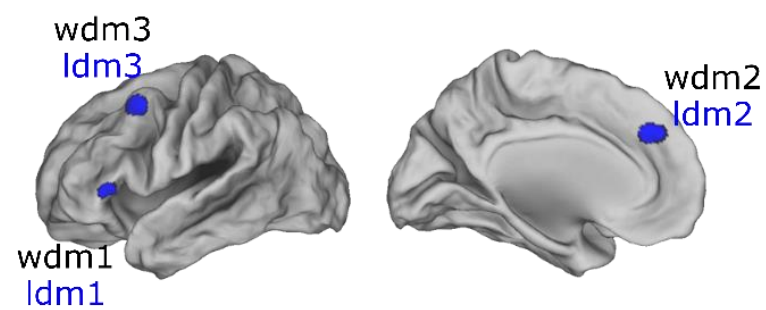

\section{DAN}
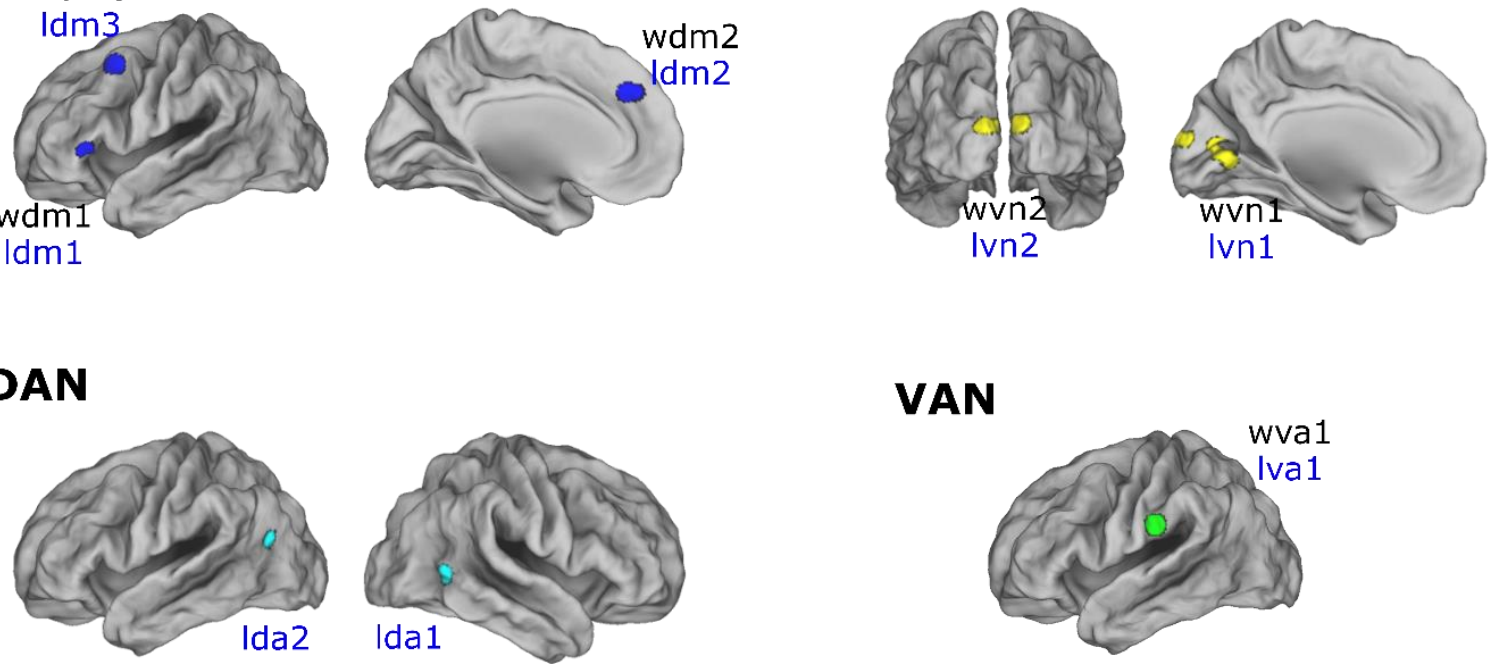
Table 2. WD-FC and local-SFC clusters of the 7 Yeo-networks that significant correlated with WM tracts lesion volumes.

MNI

coordinates

\begin{tabular}{|c|c|c|c|c|c|c|}
\hline Yeo-network & WM JHU-ICBM ROIs & WD-FC clusters & cluster ID & $\mathbf{x}$ & $\mathbf{y}$ & $\mathbf{z}$ \\
\hline \multirow[t]{3}{*}{ DMN } & Left posterior limb of internal & Left inferior frontal gyrus, triangularis & wdm1 & -54 & 32 & 2 \\
\hline & capsule & Left superior medial frontal gyrus & wdm2 & 0 & 44 & 32 \\
\hline & & Left middle frontal gyrus & wdm3 & -36 & 14 & 50 \\
\hline \multirow[t]{4}{*}{ FPN } & Left posterior limb of internal & Left middle frontal gyrus (A46) & wfp1 & -30 & 56 & 26 \\
\hline & capsule & Left middle frontal gyrus (A8vl) & wfp2 & -36 & 32 & 32 \\
\hline & & Right inferior parietal & wfp3 & 48 & -46 & 44 \\
\hline & & Left precuneus & wfp4 & 0 & -64 & 50 \\
\hline \multirow[t]{3}{*}{ SMN } & $\begin{array}{l}\text { Left posterior limb of internal } \\
\text { capsule }\end{array}$ & Right postcentral gyrus & wsm1 & 60 & -4 & 26 \\
\hline & & Left postcentral gyrus (ulhf) & wsm2 & -48 & -22 & 56 \\
\hline & $\begin{array}{l}\text { Right posterior limb of internal } \\
\text { capsule }\end{array}$ & Left postcentral gyrus (tru) & wsm3 & -30 & -34 & 74 \\
\hline VAN & $\begin{array}{l}\text { Left posterior limb of internal } \\
\text { capsule }\end{array}$ & Left supramarginal gyrus & wva1 & -54 & -22 & 26 \\
\hline \multirow[t]{2}{*}{$\mathrm{VN}$} & $\begin{array}{l}\text { Left posterior limb of internal } \\
\text { capsule }\end{array}$ & Left lingual & wvn1 & -3 & -73 & 4 \\
\hline & & Left calcarine & wvn2 & -2 & -96 & 14 \\
\hline
\end{tabular}

\begin{tabular}{|c|c|c|c|c|c|c|}
\hline \multirow[b]{2}{*}{ Yeo-network } & \multirow[b]{2}{*}{ WM JHU-ICBM ROIs } & \multirow[b]{2}{*}{ Local-SFC clusters } & \multirow[b]{2}{*}{ cluster ID } & \multicolumn{3}{|c|}{$\begin{array}{c}\text { MNI } \\
\text { coordinates }\end{array}$} \\
\hline & & & & $\mathbf{x}$ & $\mathbf{y}$ & $\mathbf{z}$ \\
\hline \multirow[t]{3}{*}{$\mathrm{DMN}$} & Left posterior limb of internal & Left inferior frontal gyrus, triangularis & $\operatorname{ldm} 1$ & -54 & 32 & 2 \\
\hline & capsule & Left superior medial frontal gyrus & $\operatorname{ldm} 2$ & 0 & 44 & 32 \\
\hline & & Left middle frontal gyrus & $\operatorname{ldm} 3$ & -36 & 14 & 50 \\
\hline \multirow[t]{4}{*}{ FPN } & Left posterior limb of internal & Left middle frontal gyrus (A8vl) & lfp1 & -42 & 26 & 33 \\
\hline & capsule & Right inferior parietal & lfp2 & 48 & -46 & 44 \\
\hline & & Left precuneus & lfp3 & 0 & -64 & 50 \\
\hline & $\begin{array}{l}\text { Left anterior limb of internal } \\
\text { capsule }\end{array}$ & Right inferior temporal & lfp4 & 66 & -34 & -16 \\
\hline \multirow[t]{5}{*}{ SMN } & $\begin{array}{l}\text { Left posterior limb of internal } \\
\text { capsule }\end{array}$ & Right postcentral gyrus & $1 \mathrm{sm} 1$ & 60 & -4 & 26 \\
\hline & & Left postcentral gyrus (ulhf) & $1 \mathrm{sm} 2$ & -48 & -16 & 56 \\
\hline & & Left postcentral gyrus (tru) & $1 \mathrm{sm} 3$ & -30 & -40 & 74 \\
\hline & $\begin{array}{l}\text { Right posterior limb of internal } \\
\text { capsule }\end{array}$ & Left postcentral gyrus (tru) & $\operatorname{lsm} 4$ & -30 & -40 & 74 \\
\hline & $\begin{array}{l}\text { Left superior fronto-occipital } \\
\text { fasciculus }\end{array}$ & Left rolandic operculum & $1 \mathrm{sm} 5$ & -42 & -16 & 20 \\
\hline \multirow[t]{2}{*}{ DAN } & $\begin{array}{l}\text { Left posterior limb of internal } \\
\text { capsule }\end{array}$ & Right middle temporal & lda1 & 60 & -58 & 2 \\
\hline & & Left middle temporal & lda2 & -48 & -70 & 20 \\
\hline VAN & $\begin{array}{l}\text { Left posterior limb of internal } \\
\text { capsule }\end{array}$ & Left supramarginal gyrus & lva1 & -60 & -22 & 26 \\
\hline \multirow[t]{2}{*}{$\mathrm{VN}$} & $\begin{array}{l}\text { Left posterior limb of internal } \\
\text { capsule }\end{array}$ & Left lingual & $\operatorname{lvn} 1$ & 0 & -70 & 2 \\
\hline & & Left calcarine & $\operatorname{lvn} 2$ & 0 & -94 & 14 \\
\hline
\end{tabular}

Brainnetome Atlas labeling (http://atlas.brainnetome.org/bnatlas.html). ulhp = upper limb, head and face region;

tru=trunk region, $A 46=$ Brodmann area 46, A8vl = ventrolateral Brodmann area 8 


\subsection{Functional connectivity: between-group differences}

Between-group comparisons, controlled for age and sex, revealed significant increased WD-FC $\left(\mathrm{F}_{1,58}=4.36, \mathrm{p}\right.$ 0.041) and local-SFC $\left(\mathrm{F}_{1,58}=4.36, \mathrm{p}=0.041\right)$ in MS patients compared to HC in the left middle frontal gyrus of DMN.

\subsection{Correlations between WD-FC and BRB-N scores}

HC exhibited significant positive correlations between BRB-N scores and WD-FC (table 3). More specifically, we found a positive correlation between left superior medial frontal gyrus of DMN and SRT-storage score, and also between left supramarginal gyrus of VAN and SRT-storage and SRTretrieval nodes. Regarding MS patients, this group showed significant negative correlations between BRB-N scores and WD-FC. First, in the DMN, the left inferior frontal gyrus was negatively correlated with SRT-storage and SPART-delay scores, and the left middle frontal gyrus was negatively correlated with SRT-retrieval score. Second, in the FPN we found negative correlations between the left middle frontal (A46) gyrus and SRT-retrieval, SPART-immediate and SPART-delay scores, the left middle frontal (A8vl) gyrus and SPART-delay score, the right inferior parietal gyrus and PASAT, SRT-retrieval and SPART-delay scores, and the left precuneus and SPART-immediate and SPARTdelay scores. Third, in the SMN, the right postcentral (ulhf) gyrus exhibits a negative correlation with SPART-immediate and SPART-delay scores, additionally, there was a negative correlation between left postcentral (tru) gyrus and SPART-immediate and SPART-delay scores. Furthermore, the left supramarginal gyrus of VAN showed a negative correlation with SPART-delay score. Finally, in the VN, we found negative correlations between left lingual gyrus and PASAT, SPART-immediate and SPART-delay scores.

To summarize, table 3 only includes those correlations with BRB-N subtests that present any statistically significant correlation with WD-FC clusters, see supplementary material for the full table. 
Table 3. Spearman's correlation between weighted degree connectivity clusters and BRB-N scores.

\begin{tabular}{|c|c|c|c|c|c|c|c|}
\hline \multirow[b]{2}{*}{$\mathrm{HC}$} & \multirow[b]{2}{*}{$(N=22)$} & \multicolumn{6}{|c|}{ Spearman's rho } \\
\hline & & PASAT & $\begin{array}{c}\begin{array}{c}\text { SRT } \\
\text { storage }\end{array} \\
\end{array}$ & $\begin{array}{c}\text { SRT } \\
\text { retrieval }\end{array}$ & $\begin{array}{c}\text { SRT } \\
\text { recovery }\end{array}$ & $\begin{array}{c}\text { SPART } \\
\text { immediate }\end{array}$ & $\begin{array}{c}\begin{array}{c}\text { SPART } \\
\text { delay }\end{array} \\
\end{array}$ \\
\hline \multirow{3}{*}{ DMN } & Left inferior frontal, triangularis & $-0,137$ & 0,171 & 0,067 & $-0,203$ & 0,158 & 0,286 \\
\hline & Left superior medial frontal & $-0,044$ & $0,444 *$ & 0,388 & 0,364 & 0,273 & 0,162 \\
\hline & Left middle frontal & 0,101 & 0,132 & 0,173 & 0,178 & 0,168 & 0,068 \\
\hline \multirow{4}{*}{ FPN } & Left middle frontal (A46) & $-0,243$ & 0,055 & $-0,128$ & 0,159 & 0,037 & $-0,085$ \\
\hline & Left middle frontal (A8vl) & $-0,090$ & 0,420 & 0,260 & 0,248 & 0,081 & $-0,028$ \\
\hline & Right inferior parietal & $-0,066$ & 0,219 & $-0,023$ & 0,100 & $-0,017$ & $-0,058$ \\
\hline & Left precuneus & 0,035 & 0,154 & 0,110 & 0,039 & 0,013 & $-0,013$ \\
\hline \multirow{3}{*}{ SMN } & Right postcentral & $-0,012$ & $-0,022$ & $-0,151$ & 0,117 & 0,121 & 0,102 \\
\hline & Left postcentral (ulhf) & 0,130 & 0,318 & 0,173 & 0,363 & 0,166 & 0,002 \\
\hline & Left postcentral (tru) & 0,425 & 0,083 & 0,122 & 0,238 & 0,117 & $-0,001$ \\
\hline VAN & Left supramarginal & 0,010 & $\mathbf{0 , 5 8 4 * *}$ & $0,515^{*}$ & 0,324 & 0,153 & 0,143 \\
\hline \multirow{2}{*}{$\mathbf{V N}$} & Left lingual & $-0,224$ & $-0,033$ & $-0,146$ & $-0,318$ & $-0,143$ & $-0,077$ \\
\hline & Bilateral cuneus & $-0,033$ & 0,088 & $-0,050$ & 0,185 & 0,052 & $-0,171$ \\
\hline$M S$ & $(N=29)$ & PASAT & $\begin{array}{c}\text { SRT } \\
\text { storage }\end{array}$ & $\begin{array}{c}\text { SRT } \\
\text { retrieval }\end{array}$ & $\begin{array}{c}\text { SRT } \\
\text { recovery }\end{array}$ & $\begin{array}{c}\text { SPART } \\
\text { immediate }\end{array}$ & $\begin{array}{c}\text { SPART } \\
\text { delay }\end{array}$ \\
\hline \multirow{3}{*}{ DMN } & Left inferior frontal, triangularis & $-0,200$ & $-0,458 *$ & $-0,281$ & $-0,355$ & $-0,321$ & $-0,498^{* *}$ \\
\hline & Left superior medial frontal & $-0,142$ & $-0,228$ & $-0,231$ & $-0,097$ & $-0,175$ & $-0,285$ \\
\hline & Left middle frontal & $-0,147$ & $-0,320$ & $-0,375^{*}$ & $-0,225$ & $-0,158$ & $-0,216$ \\
\hline \multirow{4}{*}{ FPN } & Left middle frontal (A46) & $-0,313$ & $-0,355$ & $-0,452 *$ & $-0,182$ & $-0,385^{*}$ & $-0,398 *$ \\
\hline & Left middle frontal (A8vl) & $-0,217$ & $-0,140$ & $-0,140$ & $-0,005$ & $-0,299$ & $-0,371 *$ \\
\hline & Right inferior parietal & $-0,415^{*}$ & $-0,356$ & $-0,448 *$ & $-0,184$ & $-0,319$ & $-0,409 *$ \\
\hline & Left precuneus & $-0,288$ & $-0,359$ & $-0,286$ & $-0,317$ & $-0,468^{*}$ & $-0,553 * *$ \\
\hline \multirow{3}{*}{ SMN } & Right postcentral & $-0,299$ & $-0,366$ & $-0,271$ & $-0,120$ & $-0,337$ & $-0,455 *$ \\
\hline & Left postcentral (ulhf) & $-0,208$ & $-0,275$ & $-0,240$ & $-0,206$ & $-0,430 *$ & $-0,523^{* *}$ \\
\hline & Left postcentral (tru) & $-0,188$ & $-0,246$ & $-0,361$ & $-0,185$ & $-0,115$ & $-0,172$ \\
\hline VAN & Left supramarginal & $-0,292$ & $-0,248$ & $-0,181$ & $-0,111$ & $-0,313$ & $-0,399 *$ \\
\hline \multirow{2}{*}{$\mathbf{V N}$} & Left lingual & $-0,399 *$ & $-0,256$ & $-0,257$ & $-0,209$ & $-0,444^{*}$ & $-0,438 *$ \\
\hline & Bilateral cuneus & $-0,101$ & $-0,157$ & $-0,185$ & $-0,132$ & $-0,224$ & $-0,277$ \\
\hline
\end{tabular}

**. Correlation is significant at the 0.01 level (2-tailed).

*. Correlation is significant at the 0.05 level (2-tailed).

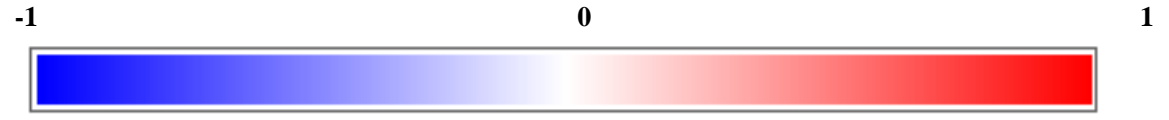

Spearman's rho 


\subsection{WD-FC and BRB-N scores correlation coefficients: between-group differences}

The Fisher r-to-z transformation revealed differences between correlation coefficients of $\mathrm{HC}$ group and MS patients in the DMN, FPN, SMN and VAN (see table 4). Firstly, in the DMN, HC group showed higher correlations than MS patients between left inferior frontal gyrus and left superior medial frontal gyrus and SRT-storage score, and left middle frontal gyrus and SRT-retrieval. Secondly, in the FPN, the HC group showed higher correlations than MS patients between left precuneus and SPART-immediate and SPART-delay scores. Thirdly, in SMN, the HC group showed a higher correlation than MS patients between right postcentral gyrus and SPART-delay. Finally, in the VAN, the HC group showed higher correlations than MS patients between left supramarginal gyrus and SRT-storage, SRT-retrieval and SPART-delay.

Table 4. Between group differences in WD-FC and BRB-N scores correlation coefficients.

\begin{tabular}{|c|c|c|c|c|c|}
\hline Yeo-network & WD-FC clusters & BRB-N subtests & $\mathbf{Z}$ & $\begin{array}{c}\text { p value } \\
\text { (one-tail) }\end{array}$ & $\begin{array}{c}\text { p value } \\
\text { (two-tail) }\end{array}$ \\
\hline \multirow[t]{4}{*}{$\overline{D M N}$} & Left inferior frontal gyrus, triangularis & SRT storage** & 2.21 & 0.0136 & 0.0271 \\
\hline & Left inferior frontal gyrus, triangularis & SPART delay** & 2.79 & 0.0026 & 0.0053 \\
\hline & Left superior medial frontal gyrus & SRT storage*** & 2.35 & 0.0094 & 0.0188 \\
\hline & Left middle frontal gyrus & SRT retrieval* & 1.89 & 0.0294 & 0.0588 \\
\hline \multirow[t]{9}{*}{ FPN } & Left middle frontal gyrus (A46) & SRT retrieval & 1.19 & 0.117 & 0.234 \\
\hline & Left middle frontal gyrus (A46) & SPART immediate & 1.47 & 0.0708 & 0.1416 \\
\hline & Left middle frontal gyrus (A46) & SPART delay & 1.11 & 0.1335 & 0.267 \\
\hline & Left middle frontal gyrus (A8vl) & SPART delay & 1.2 & 0.1151 & 0.2301 \\
\hline & Right inferior parietal & PASAT & 1.24 & 0.1075 & 0.215 \\
\hline & Right inferior parietal & SRT recovery & 1.52 & 0.0643 & 0.1285 \\
\hline & Right inferior parietal & SPART delay & 1.25 & 0.1056 & 0.2113 \\
\hline & Left precuneus & SPART immediate* & 1.72 & 0.0427 & 0.0854 \\
\hline & Left precuneus & SPART delay** & 2.02 & 0.0217 & 0.0434 \\
\hline SMN & Right postcentral gyrus & SPART delay** & 1.97 & 0.0244 & 0.0488 \\
\hline \multirow[t]{3}{*}{ VAN } & Left supramarginal gyrus & SRT storage** & 3.05 & 0.0011 & 0.0023 \\
\hline & Left supramarginal gyrus & SRT retrieval** & 2.49 & 0.0064 & 0.0128 \\
\hline & Left supramarginal gyrus & SPART delay* & 1.88 & 0.0301 & 0.0601 \\
\hline \multirow[t]{3}{*}{$\mathbf{V N}$} & Left lingual & PASAT & 0.64 & 0.2611 & 0.5222 \\
\hline & Left lingual & SPART immediate & 1.1 & 0.1357 & 0.2713 \\
\hline & Left lingual & SPART delay & 1.3 & 0.0968 & 0.1936 \\
\hline
\end{tabular}

*one-tail significant differences, **two-tail significant differences 


\subsection{Correlations between local-SFC and BRB-N scores}

As shown in table 5, HC exhibited significant positive correlations between BRB-N scores and localSFC. More specifically, we found a positive correlation between left superior medial frontal gyrus of DMN and SRT-storage score. We also found positive correlations between left middle temporal of DAN and SRT-storage and SRT-retrieval. Similarly, there were positive correlations between the left supramarginal gyrus of VAN and SRT-storage and SRT-retrieval. Regarding MS patients, this group showed significant negative correlations between BRB-N scores and WD-FC. First, in the DMN, the left inferior frontal gyrus was negatively correlated with SRT-storage and SPART-delay scores, and the left middle frontal gyrus was negatively correlated with SRT-retrieval score. Second, in the FPN we found a negative correlation between left middle frontal gyrus and SPART-delay. We also found negative correlations between right inferior parietal gyrus and PASAT, SRT-retrieval and SPARTdelay scores, in the same way, the left precuneus showed negative correlations with SPARTimmediate and SPART-delay scores. Third, in the SMN, the right postcentral (ulhf) gyrus exhibits a negative correlation with SPART-delay score, additionally, we found negative correlations between left postcentral (ulhf) gyrus and SPART-immediate and SPART-delay scores. Furthermore, the left postecentral (tru) gyrus was negatively correlated with SRT-retrieval and the left Rolandic operculum showed negative correlation with SPART-immediate and SPART-delay scores. Similarly, the right middle temporal of the DAN exhibited a negative correlation with SPART-immediate and SPARTdelay scores. In the VAN, we found a negative correlation between left supramarginal gyrus and SPART-delay score. Finally, in the VN, we found negative correlations between left lingual gyrus and PASAT, SPART-immediate and SPART-delay scores.

To summarize, table 5 only includes those correlations with BRB-N subtests that present any statistically significant correlation with local-SFC clusters, see supplementary material for the full table. 
Table 5. Spearman's correlation between local connectivity clusters and BRB-N scores.

\begin{tabular}{|c|c|c|c|c|c|c|c|}
\hline \multirow[b]{2}{*}{$\mathrm{HC}$} & \multirow[b]{2}{*}{$(N=22)$} & \multicolumn{6}{|c|}{ Spearman's rho } \\
\hline & & PASAT & $\begin{array}{c}\text { SRT } \\
\text { storage }\end{array}$ & $\begin{array}{c}\text { SRT } \\
\text { retrieval }\end{array}$ & $\begin{array}{c}\text { SRT } \\
\text { recovery }\end{array}$ & $\begin{array}{c}\text { SPART } \\
\text { immediate }\end{array}$ & $\begin{array}{c}\text { SPART } \\
\text { delay }\end{array}$ \\
\hline \multirow{3}{*}{ DMN } & Left inferior frontal, triangularis & $-0,137$ & 0,171 & 0,067 & $-0,203$ & 0,158 & 0,286 \\
\hline & Left superior medial frontal & $-0,044$ & $0,444 *$ & 0,388 & 0,364 & 0,273 & 0,162 \\
\hline & Left middle frontal & 0,101 & 0,132 & 0,173 & 0,178 & 0,168 & 0,068 \\
\hline \multirow{4}{*}{ FPN } & Left middle frontal gyrus (A8vl) & $-0,052$ & 0,310 & 0,196 & 0,050 & 0,086 & $-0,020$ \\
\hline & Right parietal inferior & $-0,066$ & 0,219 & $-0,023$ & 0,100 & $-0,017$ & $-0,058$ \\
\hline & Left precuneus & 0,035 & 0,154 & 0,110 & 0,039 & 0,013 & $-0,013$ \\
\hline & Right inferior temporal & $-0,107$ & 0,018 & $-0,199$ & 0,050 & 0,277 & 0,202 \\
\hline \multirow{5}{*}{ SMN } & Right postcentral (inferior ulhf) & $-0,012$ & $-0,022$ & $-0,151$ & 0,117 & 0,121 & 0,102 \\
\hline & Left postcentral (ulhf) & 0,130 & 0,318 & 0,173 & 0,363 & 0,166 & 0,002 \\
\hline & Left postcentral (tru) & 0,406 & 0,129 & 0,149 & 0,166 & 0,110 & 0,020 \\
\hline & Left postcentral (tru) & 0,425 & 0,083 & 0,122 & 0,238 & 0,117 & $-0,001$ \\
\hline & Left rolandic operculum & 0,143 & 0,066 & 0,067 & 0,098 & 0,210 & 0,346 \\
\hline \multirow{2}{*}{ DAN } & Right middle temporal & $-0,219$ & 0,142 & 0,240 & $-0,106$ & 0,086 & 0,148 \\
\hline & Left middle temporal & 0,140 & $0,495^{*}$ & $0,462 *$ & 0,359 & 0,317 & 0,258 \\
\hline VAN & Left supramarginal & $-0,060$ & $0,596 * *$ & $0,500 *$ & 0,275 & 0,043 & 0,023 \\
\hline \multirow{2}{*}{ VN } & Left lingual & $-0,224$ & $-0,033$ & $-0,146$ & $-0,318$ & $-0,143$ & $-0,077$ \\
\hline & Bilateral cuneus & $-0,048$ & 0,120 & 0,016 & 0,195 & 0,066 & $-0,154$ \\
\hline$M S$ & $(N=29)$ & PASAT & $\begin{array}{c}\text { SRT } \\
\text { storage }\end{array}$ & $\begin{array}{c}\text { SRT } \\
\text { retrieval }\end{array}$ & $\begin{array}{c}\text { SRT } \\
\text { recovery }\end{array}$ & $\begin{array}{c}\text { SPART } \\
\text { immediate }\end{array}$ & $\begin{array}{c}\text { SPART } \\
\text { delay }\end{array}$ \\
\hline \multirow{3}{*}{ DMN } & Left inferior frontal, triangularis & $-0,200$ & $-0,458 *$ & $-0,281$ & $-0,355$ & $-0,321$ & $-0,498 * *$ \\
\hline & Left superior medial frontal & $-0,142$ & $-0,228$ & $-0,231$ & $-0,097$ & $-0,175$ & $-0,285$ \\
\hline & Left middle frontal & $-0,147$ & $-0,320$ & $-0,375 *$ & $-0,225$ & $-0,158$ & $-0,216$ \\
\hline \multirow{4}{*}{ FPN } & Left middle frontal gyrus (A8vl) & $-0,239$ & $-0,212$ & $-0,120$ & $-0,087$ & $-0,292$ & $-0,386 *$ \\
\hline & Right inferior parietal & $-0,415^{*}$ & $-0,356$ & $-0,448 *$ & $-0,184$ & $-0,319$ & $-0,409 *$ \\
\hline & Left precuneus & $-0,288$ & $-0,359$ & $-0,286$ & $-0,317$ & $-0,468 *$ & $-0,553 * *$ \\
\hline & Right inferior temporal & $-0,253$ & $-0,332$ & $-0,247$ & $-0,118$ & $-0,195$ & $-0,302$ \\
\hline \multirow{5}{*}{ SMN } & Right postcentral (inferior ulhf) & $-0,299$ & $-0,366$ & $-0,271$ & $-0,120$ & $-0,337$ & $-0,455 *$ \\
\hline & Left postcentral (ulhf) & $-0,208$ & $-0,275$ & $-0,240$ & $-0,206$ & $-0,430 *$ & $-0,523 * *$ \\
\hline & Left postcentral (tru) & $-0,223$ & $-0,270$ & $-0,368 *$ & $-0,229$ & $-0,176$ & $-0,263$ \\
\hline & Left postcentral (tru) & $-0,188$ & $-0,246$ & $-0,361$ & $-0,185$ & $-0,115$ & $-0,172$ \\
\hline & Left rolandic operculum & $-0,337$ & $-0,181$ & $-0,251$ & $-0,187$ & $-0,365$ & $-0,427 *$ \\
\hline \multirow{2}{*}{ DAN } & Right middle temporal & $-0,211$ & $-0,140$ & $-0,140$ & $-0,062$ & $-0,393 *$ & $-0,443 *$ \\
\hline & Left middle temporal & $-0,120$ & $-0,128$ & $-0,132$ & $-0,094$ & $-0,032$ & $-0,123$ \\
\hline VAN & Left supramarginal & $-0,329$ & $-0,196$ & $-0,148$ & $-0,019$ & $-0,324$ & $-0,381 *$ \\
\hline \multirow{2}{*}{$\mathbf{V N}$} & Left lingual & $-0,399 *$ & $-0,256$ & $-0,257$ & $-0,209$ & $-0,444^{*}$ & $-0,438 *$ \\
\hline & Bilateral cuneus & $-0,063$ & $-0,162$ & $-0,222$ & $-0,143$ & $-0,186$ & $-0,232$ \\
\hline & \multicolumn{7}{|c|}{$\begin{array}{l}* \text { *. Correlation is significant at the } 0.05 \text { level (2-tailed). } \\
* * \text {. Correlation is significant at the } 0.01 \text { level (2-tailed). }\end{array}$} \\
\hline & -1 & 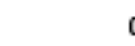 & & & & 1 & \\
\hline
\end{tabular}




\subsection{Local-SFC and BRB-N scores correlation coefficients: between-group differences}

The Fisher r-to-z transformation revealed differences between correlation coefficients of HC group and MS patients in the DMN, FPN, SMN, DAN and VAN (see table 6). Firstly, in the DMN, HC group showed higher correlations than MS patients between left inferior frontal gyrus and SRT-storage and SPART-delay scores. HC also showed a higher correlation between left superior medial frontal gyrus and SRT-storage score, as well as between left middle frontal gyrus and SRT-retrieval score. Secondly, in the FPN, the HC group showed higher correlations than MS patients between left precuneus and SPART-immediate and SPART-delay scores. Thirdly, in SMN, the HC group showed a higher correlation than MS patients between right postcentral gyrus and SPART-delay score. Additionally, HC group showed higher correlations between left postcentral gyrus (ulhf) and SPART-immediate and SPART-delay scores. HC group also exhibits a higher correlation than MS group between left Rolandic operculum and SPART-delay score. Fourthly, HC group showed higher correlations than MS group between left middle temporal gyrus and SRT-storage and SRT-retrieval scores, as well as HC group exhibits higher correlations than MS group between right middle temporal gyrus and SPART-immediate and SPART-delayed scores. Finally, in the VAN, the HC group showed higher correlations than MS group between left supramarginal gyrus and SRT-storage and SRT-retrieval scores. 
Table 6. Between group differences in local-SFC and BRB-N scores correlation coefficients.

\begin{tabular}{|c|c|c|c|c|c|}
\hline Yeo-network & Local-SFC cluster & BRB-N subtests & $\mathbf{Z}$ & $\begin{array}{c}\text { p value } \\
\text { (one-tail) }\end{array}$ & $\begin{array}{c}\text { p value } \\
\text { (two-tail) }\end{array}$ \\
\hline \multirow[t]{4}{*}{ DMN } & Left inferior frontal gyrus, triangularis & SRT storage** & 2.21 & 0.0136 & 0.0271 \\
\hline & Left inferior frontal gyrus, triangularis & SPART delay $* *$ & 2.79 & 0.0026 & 0.0053 \\
\hline & Left superior medial frontal gyrus & SRT storage ${ }^{* *}$ & 2.35 & 0.0094 & 0.0188 \\
\hline & Left middle frontal gyrus & SRT retrieval* & 1.89 & 0.0294 & 0.0588 \\
\hline \multirow[t]{6}{*}{$\overline{\text { FPN }}$} & Left middle frontal gyrus (A8vl) & SPART delay & 1.26 & 0.1038 & 0.2077 \\
\hline & Right inferior parietal & PASAT & 1.24 & 0.1075 & 0.215 \\
\hline & Right inferior parietal & SRT recovery & 1.52 & 0.0643 & 0.1285 \\
\hline & Right inferior parietal & SPART delay & 1.25 & 0.1056 & 0.2113 \\
\hline & Left precuneus & SPART immediate* & 1.72 & 0.0427 & 0.0854 \\
\hline & Left precuneus & SPART delay** & 2.02 & 0.0217 & 0.0434 \\
\hline \multirow[t]{5}{*}{ SMN } & Right postcentral gyrus & SPART delay** & 1.97 & 0.0244 & 0.0488 \\
\hline & Left postcentral gyrus (ulhf) & SPART immediate** & 2.08 & 0.0188 & 0.0375 \\
\hline & Left postcentral gyrus (ulhf) & SPART delay* & 1.93 & 0.0268 & 0.0536 \\
\hline & Left postcentral gyrus (tru) & SRT retrieval* & 1.78 & 0.0375 & 0.0751 \\
\hline & Left rolandic operculum & SPART delay* & 2.71 & 0.0034 & 0.0067 \\
\hline \multirow[t]{4}{*}{$\overline{\text { DAN }}$} & Left middle temporal & SRT storage** & 2.22 & 0.0132 & 0.0264 \\
\hline & Left middle temporal & SRT retrieval** & 2.1 & 0.0179 & 0.0357 \\
\hline & Right middle temporal & SPART immediate* & 1.66 & 0.0485 & 0.0969 \\
\hline & Right middle temporal & SPART delayed $* *$ & 2.07 & 0.0192 & 0.0385 \\
\hline \multirow[t]{3}{*}{ VAN } & Left supramarginal gyrus & SRT storage** & 2.92 & 0.0018 & 0.0035 \\
\hline & Left supramarginal gyrus & SRT retrieval* & 2.31 & 0.0104 & 0.0209 \\
\hline & Left supramarginal gyrus & SPART delay & 1.81 & 0.0351 & 0.0703 \\
\hline \multirow[t]{3}{*}{$\overline{\mathrm{VN}}$} & Left lingual & PASAT & 0.64 & 0.2611 & 0.522 \\
\hline & Left lingual & SPART immediate & 1.1 & 0.1357 & 0.2713 \\
\hline & Left lingual & SPART delay & 1.3 & 0.0968 & 0.1936 \\
\hline
\end{tabular}

*one-tail significant differences, **two-tail significant differences 


\section{Discussion}

The present research focused on studying topological specific associations between WM-lesions and changes in FC in cognitively preserved RR-MS patients using two graph theory measures: a) WD that quantifies the strength of connections between a given node and every other node (Rubinov \& Sporns, 2010), and b) modularity analysis based on triangle motifs that allows to independently describe the modularity segregation of FC in local connectivity (connection between neighboring nodes) and distributed connectivity (connections between distant nodes) (Diez \& Sepulcre, 2018). These analyses allowed us to investigate the relationship between specific FC changes and neuropsychological declines in MS.

One of the major findings of this study was that WM lesions detected in the internal capsule were consistently associated with FC changes in MS patients. Specifically, we observed that an increase in lesion burden in the left posterior limb of the internal capsule is associated with an increase WD-FC in several regions of different resting state networks, including DMN, FP, SMN, VAN and VN. The internal capsule is surrounded by deep nuclei, closely involved in MS pathology, such as basal ganglia and thalamus. This structure is divided into three components, the anterior limb, the genu and the posterior limb. The posterior limb contains thalamocortical and corticospinal fibers, which have been shown to be related with cognitive impairment (Ricigliano et al., 2021; Sepulcre et al., 2009; Zhou et al., 2016) and physical disability in MS (Bonzano et al., 2014; DeLuca et al., 2004; Ganter et al., 1999; Spain et al., 2009).

In order to identify whether the observed changes in FC correspond to local connectivity or distributed connectivity, we performed a modularity analysis (SFC). Results revealed that the increase FC associated with lesions in the internal capsule corresponded to disrupted local connectivity but not distributed connectivity. Likewise, modularity analysis revealed increased local-SFC in the bilateral middle temporal of the DAN that had not been observed by WD-FC analysis.

Regarding the relationship between the FC changes and the performance on neuropsychological tests, we found that the increases in WD-FC and local-SFC are mainly associated with lower PASAT, SRT and SPART scores. The PASAT is a neuropsychological test that measures information processing speed and working memory, while the SRT and SPART tests measure learning and long-term memory capacity for verbal and visuospatial domains, respectively. These cognitive functions are the most affected in MS patients, even in early stages of the disease (Bergendal et al., 2007; Chiaravalloti \& DeLuca, 2008; Janculjak et al., 2002; Langdon, 2011). At this respect, it is important to note that MS patients included in this study are in early stages of the disease (mean years disease duration $7.93 \pm 4.85$ ), as well as they are cognitively preserved according to the criterion of cognitive impairment defined as performance at least 1.5 standard deviations below the mean of a normative sample. Bearing this in mind, it is interesting to discuss the implications of our results in the context of the inverse U-curve hypothesis (Fleischer et al., 2019; Tewarie et al., 2018), which proposes an initial increase FC 
associated to structural disconnection, that seems to be related with compensatory mechanisms (Basile et al., 2014; Fleischer et al., 2017; Muthuraman et al., 2016; Rocca, Valsasina, et al., 2010), which reach their limits due to the built-up damage resulting in decrease in overall FC (Fleischer et al., 2019; Schoonheim, Meijer, et al., 2015; Tewarie et al., 2018). Nevertheless, there is controversy about the interpretation of increases in FC observed at early stages of the disease (Tahedl et al., 2018). On the one hand, these FC changes could be considered as compensatory mechanisms (active changes), in other words, an attempt of the brain to maintain or restore function. On the other hand, the increases in FC can be interpreted as epiphenomenal (passive changes), a by-product of the lesions. The previously mentioned compensatory interpretation has been mainly reported in studies that found increases in FC in task-specific regions (Louapre et al., 2014; Zhong et al., 2016), such as studies focus on cognitive rehabilitation programs (Aguirre et al., 2021; Bonavita et al., 2015; Filippi, Riccitelli, et al., 2012; Leavitt, Wylie, Girgis, et al., 2014), whereas studies reporting nonspecific increases in FC have associate them with maladaptive processes (Schoonheim, Hulst, et al., 2015; Zhong et al., 2017). Accordingly, our findings could be interpreted on the basis of these two hypotheses. As for the first hypothesis, considering that only cognitive preserved MS patients were included in the study and also that these patients compared with HC did not show any significant difference in the execution of neuropsychological tests, the increases in FC could be considered as compensatory mechanisms, attempting to maintain the normal functional level. Regarding the second hypothesis, our results would be in line with the maladaptive processes interpretation. Firstly, because we are exploring changes in FC related to a pathological disease process, i.e., WM lesions. Secondly, because the increases in FC are associated with worse performance in MS patients but no in HC. This seems to indicate that this methodology allows exploring subtle changes in FC that may take place in early stages of the disease, when cognitive impairment has not still been possible to determine by clinical criteria.

Nevertheless, to know whether increases in FC are explained by compensatory mechanisms or maladaptive processes secondary to WM lesions, it would be necessary to explore heterogeneous groups of patients with different degrees of cognitive impairment. Future studies need to address this limitation and to clarify if increases in local FC secondary to lesion load observed at early stages of the disease (Muthuraman et al., 2016) is followed by a disruption of global information transfer and decreased connectivity in long-range paths that may be the result of the disseminated focal lesions and ongoing cognitive decline (Gamboa et al., 2014; Schoonheim, Meijer, et al., 2015; Tahedl et al., 2018).

The novelty of this work is the combined used of graph theory to explore the underlying network-wise mechanisms of brain dysconnectivity changes associated with the topological impact of WM-lesions on FC. Their contemporary application in longitudinal studies and with different MS phenotypes may provide complementary information useful to understand the pathological mechanisms underlying cognitive impairment in MS. 


\section{Conclusions}

Our study shows that in cognitive preserved MS patients, lesions in the left posterior limb of the internal capsule are consistently associated with increases in FC, concretely with local-FC. Likewise, the increases in FC were related with lower scores in neuropsychological tests that measure the most affected cognitive domains in MS. The increases in FC can be interpreted as compensatory mechanisms or maladaptive processes secondary to WM-lesions. Further research with MS patients of heterogeneous phenotypes and different degree of cognitive impairment is needed in order to clarify the interpretation of the FC changes. Nevertheless, the combined use of graph theory measures and WM-lesion location seems to be a promising tool to understand the development of MS pathology and its effect on brain functionality. 


\title{
Study II
}

Repeated working memory training improves neural efficiency and cognitive performance in multiple sclerosis patients and healthy controls

\author{
Naiara Aguirre ${ }^{1}$, Álvaro Javier Cruz-Gómez ${ }^{1}$, Anna Miró-Padilla ${ }^{1}$, Elisenda Bueichekú ${ }^{1}$, Ricardo Broseta \\ Torres $^{2}$, César Ávila ${ }^{1}$, Carla Sanchis-Segura ${ }^{1}$, Cristina Forn ${ }^{1}$ \\ ${ }^{1}$ Jaume I University. Department of Basic, Clinic and Psychobiology. Neuropsychology and Functional \\ Neuroimaging group \\ ${ }^{2}$ ERESA, medic group. Valencia, Spain
}

doi: $10.1155 / 2019 / 2657902$ 


\section{Abstract}

Background/Objective: To explore the effectiveness of a specific working memory (WMF) training program in MS patients and healthy controls (HC).

Method: 29 MS patients and 29 matched HC were enrolled in the study. MS and HC were randomly split into two groups: Non-training groups (15 HC /14 MS) and training groups (14 HC / $15 \mathrm{MS}$ ). Training groups underwent adaptive n-back training (60 min/day; 4 days). fMRI was used to monitor brain activity during $\mathrm{n}$ back performance (conditions: 0-back, 2-back, and 3-back) at 3 time points: 1) baseline, 2) post-training (+7days), and 3) follow-up (+35days).

Results: In post-training and follow-up fMRI sessions, trained groups (HC and MS patients) exhibited significant reaction time (RT) reductions and increases in correct responses (CRs) during 2-back and 3-back performance. This cognitive improvement was accompanied by a decrease in brain activation in the WMF fronto-parietal network. The two effects were significantly correlated.

Conclusions: After WMF training, both MS and HC participants showed cognitive improvement made possible by neuroplastic processes that enhanced neural efficiency.

Keywords: working memory, n-back task, cognitive rehabilitation, functional MRI 


\section{Introduction}

Cognitive impairment is present in 40-65\% of Multiple Sclerosis (MS) patients (Benedict \& Zivadinov, 2011). Although the neuropsychological profile varies among patients, there is evidence that the functions mainly affected in this clinical population, even in the early stages of the disease, are information processing speed, attention, and working memory (WMF) (Chiaravalloti \& DeLuca, 2008; Potagas et al., 2008). A decline in cognitive functions has a negative impact on patients' daily lives and quality of life (Chiaravalloti \& DeLuca, 2008). Consequently, numerous studies have been carried out in recent years to explore the potential effects of cognitive interventions focused on improving cognition in MS patients.

In this regard, several studies using fMRI have provided positive evidence about the presence of neural plasticity in MS patients that helps to conceal the disease's clinical expression, at least in some phases of the disease (Chiaravalloti et al., 2015; Cruz-Gómez et al., 2014; Forn et al., 2006). However, the counteracting effects of spontaneous neuroplasticity are limited and transitory, and they are ultimately overcome by the disease's progression (Tomassini et al., 2012).

As initial evidence of this concept, Sastre Garriga et al. (2010) showed that neuropsychological training resulted in general cognitive improvement in MS patients, with an increase in activity in cerebellar areas. After this initial study, other researchers showed the efficacy of neuropsychological interventions in improving memory (Chiaravalloti et al., 2012; Ernst et al., 2012; Parisi, Rocca, Valsasina, et al., 2014), attention, processing information speed, and executive functions (Bonavita et al., 2015; Campbell et al., 2016; De Giglio et al., 2015; Leavitt, Wylie, Girgis, et al., 2014; Parisi, Rocca, Mattioli, et al., 2014) in MS patients. In general, the results of these studies revealed greater cerebral activation or higher functional connectivity in MS patients after completing the training program, which in some cases was positively correlated with improvements in cognitive performance. However, as a recent review pointed out (Mitolo et al., 2015), the results of these studies are inconclusive, perhaps due to the heterogeneity of the selected participants, the diversity and lack of specificity of the rehabilitation approaches used, and other methodological weaknesses (e.g. the selection of outcome measures).

Taking these limitations into account, we designed a study to test the effectiveness of a WMF training program in a group of MS patients. We focused our cognitive training on WMF functions because it has been suggested that cognitive rehabilitation programs targeting specific cognitive domains could maximize their effectiveness; and improving functions that play central roles in the cognitive architecture (as in the case of WMF and cognitive executive control processes) will maximize the applicability of the intervention's effect (i.e. generality) (Mitolo et al., 2015). From the experimental tasks that could be used to assess and rehabilitate WMF, we chose the n-back because it has been found to improve WMF (Covey et al., 2018; Miró-Padilla et al., 2018). Taking into account that WMF processes involve the maintenance and fast manipulation of 
information, training WMF processes can also lead to an improvement in information processing speed. Finally, regarding participant selection, we recruited HC and a very homogenous sample of RRMS patients without signs of cognitive deficits. This experimental design was selected in order to: 1) ensure that there were no initial (baseline assessment) cerebral differences in MS patients secondary to their pathology; 2) allow between but also within-group comparisons that could corroborate possible WMF improvement; and 3) assess whether the cerebral changes associated with WMF training are similar or different in HC and MS patients.

Taking into account previous studies reporting that repeated task training produces performance enhancements and brain activity decreased in HC (Miró-Padilla et al., 2018), the aim of the present study was to explore whether WMF training could improve behavioural performance in MS patients and decrease cerebral activity in WMF networks indicative of neural efficiency.

\section{Material and methods}

\subsection{Participants}

Twenty-nine right-handed patients diagnosed with clinically definitive RR MS according to McDonald's criteria (Polman et al., 2011) were selected for the study, and twenty-nine right-handed participants with no neurological or psychiatric dysfunction made up the control group (HC). Participants were randomly subdivided into four groups: $14 \mathrm{MS}$ untrained group (MSu), $15 \mathrm{HC}$ untrained group ( $\mathrm{HCu}$ ), $15 \mathrm{MS}$ trained group (MSt), and $14 \mathrm{HC}$ trained group $(\mathrm{HCt})$. All participants received remuneration for completing the study. The Ethical Committee of Universitat Jaume I approved the research project. All participants gave informed written consent prior to participation.

All participants were assessed with: 1) the Brief Repeatable Battery of Neuropsychological Tests (BRB-N), validated for the Spanish population (Sepulcre, Vannotti, et al., 2006); the Matrix Reasoning Subtest of the Wechsler Adult Intelligence Scale (WAIS III) to assess the intelligence quotient (IQ); 2) the Fatigue Severity Scale (FSS); and 3) the Beck Depression Inventory (BDI). In addition, patients were neurologically assessed using the Expanded Disability Status Scale (EDSS). Moreover, assessment also included three fMRI sessions: base- line session ( $\mathrm{S} 1$ ), post-training session (S2; 7 days later), and follow-up session (S3; 42 days later). In S3, due to technical problems, the data of $7 \mathrm{MSt}$ and $9 \mathrm{MSu}$ participants were lost. Therefore, we randomly dropped data from $7 \mathrm{HCt}$ and $8 \mathrm{HCu}$ in order to compare similarly sized groups $(8 \mathrm{HCu} / 6 \mathrm{HCt} / 7 \mathrm{MSu} / 6$ MSt). See Figure 1 for a schematic description of the experimental design.

Figure 1. Flow diagram of the phases of the study. 


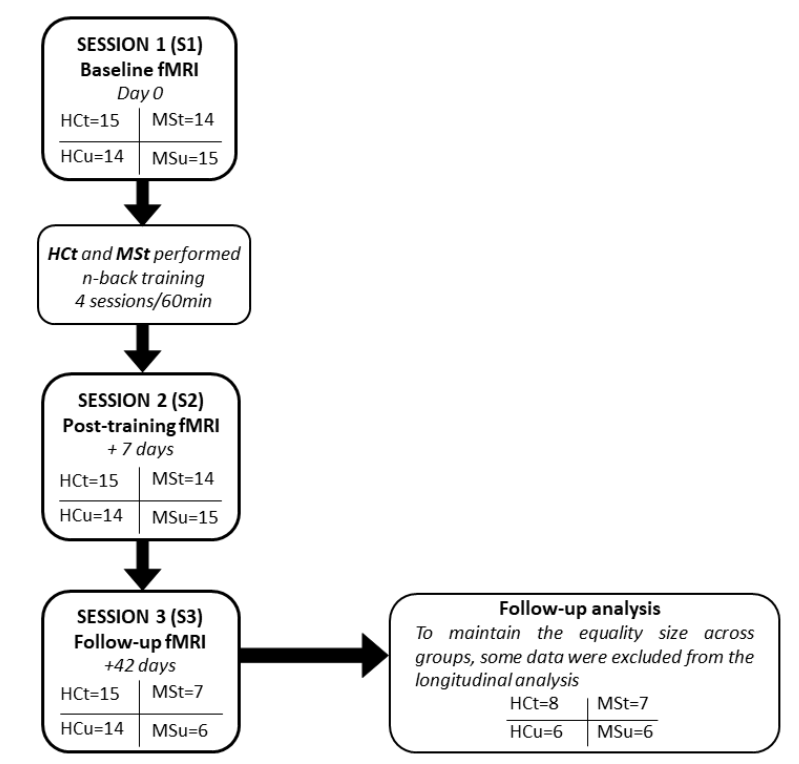

\subsection{MRI acquisition}

MRI data were collected on a $1.5 \mathrm{~T}$ scanner (Siemens Symphony, Erlangen, Germany) in the following order in the three sessions: 1) Anatomical 3D MPRAGE volumes were acquired, using a T1-weighted gradient echo pulse sequence $\left(\mathrm{TR}=2200 \mathrm{~ms}\right.$; $\mathrm{TE}=3 \mathrm{~ms}$; flip angle $=15^{\circ}$; matrix $=256 \times 256 \times 160$; voxel $\left.=1 \times 1 \times 1 \mathrm{~mm}\right)$ and for MS patients a FLAIR sequence $\left(\mathrm{TR}=6000 \mathrm{~ms} ; \mathrm{TE}=354 \mathrm{~ms} ;\right.$ flip angle $=180^{\circ}$; matrix $=196 \times 256 \times 160$; voxel $=1.05 \times 1.05 \times 1 \mathrm{~mm}$ ); 2) fMRI data during n-back were acquired with a gradient-echoT2*-weighted echoplanar MR sequence covering the entire brain $\left(\mathrm{TR}=2500 \mathrm{~ms}\right.$; $\mathrm{TE}=49 \mathrm{~ms}$; matrix $=64 \times 64 \times 28$; flip angle $=90^{\circ}$; voxel $=3.5 \times 3.5 \times 3.5$; slice gap $=4.41 \mathrm{~mm})$. A total of 260 volumes were recorded.

\subsection{N-back fMRI task}

The n-back adapted for fMRI has been described in previous studies (Miró-Padilla et al., 2018; Polman et al., 2011; Sepulcre, Vannotti, et al., 2006; Zou et al., 2013). Briefly, the n-back task used in this fMRI study consisted of a block task with 3 conditions: 0-back as a baseline control task and 2 and 3-back as a WMF task. Visual stimuli comprised 15 capital letters from the alphabet; there were 270 stimuli in the entire task, and 54 were targets. Any letter could be the target in 2 and 3-back, but the $\mathrm{X}$ was the target in 0-back. Thus, during 0back, subjects were instructed to press the "yes" button when the X target letter was presented on the screen, and the "no" button when any other letters were presented. During the 2 and 3-back tasks, participants were instructed to press the "yes" button when the letter presented on the screen matched the one presented 2 or 3 items back, and press "no" when they saw no target letters on the screen. Manual responses were given with the right hand, responding to targets with their thumb and to non-targets with their forefinger. The task was composed of 9 blocks pseudo randomly presented, three for each level. Each block lasted 60.7s and consisted of $200 \mathrm{~ms}$ of a blank screen, followed by 30 (6 target) consecutive trials of single-letter stimuli presented for 
$500 \mathrm{~ms}$ with a $1500 \mathrm{~ms}$ inter stimulus interval, with 500ms of a blank screen at the end of each block. In addition, each block also included $800 \mathrm{~ms}$ of a fixation cross and $2000 \mathrm{~ms}$ of the instruction display indicating the task difficulty of the block. The total duration of the task was $11 \mathrm{~min}$. Before fMRI acquisition, subjects received oral instructions about how to do the task, and they performed 5 min practice. For a more specific description of the task, see Miró-Padilla et al. (2018).

Visual stimuli were presented electronically using E-Prime software (Psychology Software Tools, Pittsburgh, PA), professional version 2.0, installed in a Hewlett-Packard portable workstation (screen-resolution $800 \mathrm{x}$ 600, refresh rate of $60 \mathrm{~Hz}$ ). Participants watched the laptop screen through MRI-compatible goggles (VisuaStim, Resonance Technology, Inc., Northridge, CA, USA). Participants had to make "yes" or "no" motor responses during the task that were collected via MRI-compatible response-grips (NordicNeuroLab, Bergen, Norway). The E-Prime's logfile saved the correct responses (CRs) and reaction times (RTs) to each stimulus for each participant.

\subsection{N-back training protocol}

Two days after S1, the trained groups came to the university for 4 sessions (duration: $60 \mathrm{~min} / \mathrm{session}$ ) of $\mathrm{n}$ back training. These sessions were conducted, following the procedure reported by Miró-Padilla et al. (2018), by three experimenters blinded to group (HC/ MS) assignment. Trained groups performed four training sessions of 60 min each on four consecutive days. Training sessions were distributed in two phases. During the first phase, participants performed WMF training, which consisted of an adaptive $n$-back paradigm adapted from Jaeggi et al. (2008) for 50 minutes. In this phase, participants performed three runs, each composed of eight blocks that varied in WMF load (1-back, 2-back and 3-back). For motivational reasons (Schneiders et al., 2012), the training always started at the low level, that is, with a 1-back load, but the level of n-back of the subsequent block was based on the participant's performance on the previous block. Thus, if the participant had at least 90\% CRs, the WMF load increased one level (e.g. 90\% performance on 2-back tasks increased to 3-back). If the CRs during the block were below $80 \%$, in the subsequent block the WMF load decreased one level (e.g. from 2-back to 1-back). In all other cases, the n-level remained constant (Salminen et al., 2012). As in the n-back fMRI task, participants were instructed to give manual responses only with their right hand, responding to targets with their thumb and to non-targets with their forefinger. Feedback was introduced after each response for a few seconds, as a colored circle at the corner of the screen: green meant a correct answer, a red circle represented an error, and blue meant missing responses. Moreover, at the end of each block, subjects also received additional information about the percentage of their CRs and the RT average of their responses. In the test phase, participants performed eight blocks of the 2 and 3-back task. Subjects had no feedback during this time. Their results on this test were useful to evaluate their progress on n-back, and they are reported below and in Supplementary Table 1. The non-trained group did nothing during the training 
period. See also supplementary material and Supplementary Figure 1 for a more specific description of the nback training protocol.

\subsection{Neuroimaging analysis}

Preprocessing and statistical analysis of fMRI data were conducted with SPM12 (Wellcome Trust Centre for Neuroimaging, London, UK). The personnel responsible for fMRI data analysis were also blinded to the participants' group assignment. Preprocessing included the following steps: head motion correction, where the functional images were realigned and resliced to fit the mean functional image. No participant had a head motion of more than $1.5 \mathrm{~mm} /$ degrees in any of the six directions during functional data recording. Afterwards, the anatomical image (T1-weighted) was co-registered to the mean functional image, and the transformed anatomical image was then segmented. The functional images were spatially normalized to the MNI (Montreal Neurological Institute, Montreal, Canada) space with a $3 \mathrm{~mm} 3$ resolution, and spatially smoothed with an isotropic Gaussian kernel of 8 mm FWHM (Full-Width at Half-Maximum).

A general lineal model was used in the first level of analysis to obtain the task activation maps for each WMF condition, compared to the control condition: "2-back>0-back", "3-back>0-back". The BOLD signal was estimated by convolving the stimuli onset with the canonical hemodynamic response function. Six motion realignment parameters were included as covariates of no interest in order to explain signal variations due to head motion. A high-pass filter (128s) was applied to the functional data to eliminate low-frequency components.

In a second level of analysis, whole-brain one-sample t-tests were conducted to study the brain regions involved in each condition (2-back>0-back and 3-back>0-back) for each group, using the fMRI data collected in S1. S1 data were also used to perform a Full Factorial ANCOVA (sex and age as a covariates) to examine the initial equality of the brain responses between the groups and the assigned experimental condition (Group x Training). Additionally, we used a Flexible Factorial design, with sex and age as covariates (repeatedmeasures ANCOVA), to study immediate effects of the training on the brain, comparing S2 to S1. An interaction analysis (Group x Training x Session) was carried out separately for each experimental condition (2-back and 3-back). Due to the data loss in S3, a separate Flexible Factorial analysis was performed to study the stability of the training effects 35 days after the training session (S1 vs S3). Results were p<0.05 FWEcluster corrected with an auxiliary threshold of $p<0.001$. Finally, partial correlations with sex and age as covariates were performed to investigate the possible relation- ship between participants' performance (the difference obtained between S1 vs S2 in mean CRs and mean RTs) and the mean significant BOLD signal, i.e., the corresponding Eigen values for the significant clusters obtained in the prior Flexible Factorial analyses for each condition (2 and 3-back). 
In all patients, T1-hypointense lesions were identified and filled using the "LST: Lesion Segmentation Tool" (https://www.applied-statistics.de/lst.html), calculating the lesion probability maps with the Lesion growth algorithm (LGA) (Schmidt et al., 2012). The brain parenchymal fraction (BPF) for all the participants was obtained from the 3D image by following the SPM12 software (Wellcome Trust Centre for Neuroimaging, London, UK) segmentation step, according to the procedure described by Sanfilipo et al. (2004).

\subsection{Behavioral analysis}

SPSS 22 (IBM Corp) was used to process the neuropsychological and clinical data presented in Table 1 and the fMRI data obtained in each group in each session. 2x2 ANCOVAs [Group (HC vs MS) $x$ Training (untrained vs trained); covariates: sex and age] were conducted to assess potential baseline differences among groups and training conditions. Additionally, 2x2x3 mixed model ANCOVAs [Group (HC vs MS) x Training (untrained vs trained) x Session (S1 vs. S2 vs. S3); covariates: sex and age] were conducted for each variable (CRs and RTs) and for each condition (2-back and 3-back). Longitudinal behavioral data loss was lower than that for neuroimaging data; thus, we lost 6 participants' data in all (2 participants in the HCt group, and 2 in the MSu group, 2 in the MSu group -see also Figure 1). For this reason, we decided to use the mean CRs and RTs of each group in S3 to replace the S3 missing values. All the analyses were followed by Bonferroni posthoc tests, which provide p-values adjusted for multiple comparisons.

Finally, with the test phase data from the training, repeated-measures ANCOVAs (age and sex as covariates) for each n-back condition (2-back and 3-back) and performance measures (CRs and RTs) were conducted separately, with group (HCt and MSt) as between-subject factor and tests from the training sessions (T1 vs T2 vs T3 vs T4) as within-subjects factor. To evaluate the improvement due to the cognitive training, we carried out paired t-tests, comparing Test 1 with Test 4. 


\section{Results}

\subsection{Demographic and neuropsychological results}

As Tables $1 \mathrm{a}$ and $\mathrm{b}$ show, no statistically significant differences between groups ( $\mathrm{HCu}, \mathrm{HCt}$, MSu and MSt) were observed for age, gender, cognitive performance, or fatigue scores. Conversely, statistically significant differences between groups were observed for BDI scores and, as expected, BPF volume.

\subsection{Imaging data results}

As Supplementary Figure 1 reveals, during the performance of the 2 and 3-back tasks in the baseline session (S1), all participants showed brain activations in fronto-parietal areas related to WMF processes, and no differences between groups were observed. As Figure 2A and Supplementary Figure 2A show, when the activations associated with the performance of the 2-back task in S2 were compared to those observed in S1, both (HC and MS) trained groups showed lower activations in the fronto-parietal network, compared to the untrained groups.

As specified in Table 2, during the 2-back task, trained participants showed reduced activation in the left postcentral gyrus, right frontal gyrus, angular and supramarginal gyrus, and bilateral inferior parietal lobule. Similar deactivations in trained groups were observed during the performance of the 3-back task (Figure 2B, Supplementary Figure 2B), but in this case, these deactivations were restricted to bilateral frontal areas, and they were especially remarkable in bilateral supplementary motor areas and bilateral middle and superior

frontal gyrus (See Table 2). fMRI results comparing S3 to S1 are reported in the supplementary material (See Supplementary Table 2 and Supplementary Figure 3). 
Table 1A. Demographic and clinical data of all participants

\begin{tabular}{|c|c|c|c|c|c|}
\hline Results & $\begin{array}{l}\mathbf{H C}_{\mathbf{U}}(\mathbf{n}=\mathbf{1 5}) \\
(\text { mean } \pm \mathrm{SD})\end{array}$ & $\begin{array}{r}\mathbf{H C}_{\mathbf{T}}(\mathbf{n}=\mathbf{1 4}) \\
(\text { mean } \pm \mathrm{SD})\end{array}$ & $\begin{array}{r}\mathbf{M S}_{\mathbf{U}}(\mathbf{n}=\mathbf{1 4}) \\
(\text { mean } \pm \mathrm{SD})\end{array}$ & $\begin{array}{r}\mathbf{M S}_{\mathbf{T}}(\mathbf{n}=\mathbf{1 5}) \\
(\text { mean } \pm \text { SD })\end{array}$ & Differences \\
\hline Age & $\begin{array}{l}34.13 \pm 6.07 \\
(25-45)\end{array}$ & $\begin{array}{l}31.21 \pm 8.72 \\
(24-50)\end{array}$ & $\begin{array}{l}36.14 \pm 5.97 \\
(22-46)\end{array}$ & $\begin{array}{l}35.80 \pm 7.3 \\
(22-46)\end{array}$ & $\begin{array}{l}\text { Training: } F_{1,54}=0.762, p=0.387 \\
\text { Group: } F_{1,54}=3.115, p=0.083 \\
\text { Interaction: } F_{1,54}=0.475, p=0.494\end{array}$ \\
\hline Gender (men/women) & $9 / 6$ & $6 / 8$ & $3 / 11$ & $7 / 8$ & $\chi_{(3)}^{2}=4.51, p=0.212$ \\
\hline Educational level (1-6 levels) & $3.73 \pm 1.28$ & $4.71 \pm 0.83$ & $3.71 \pm 1.49$ & $3.73 \pm 1.71$ & $\begin{array}{l}\text { Training: } F_{1,54}=1.925, p=0.171 \\
\text { Group: } F_{1,54}=1.925, p=0171 \\
\text { Interaction: } F_{1,54}=1.781, p=0.188\end{array}$ \\
\hline EDSS & - & - & $1.80 \pm 1.70$ & $1.67 \pm 1.51$ & $\mathrm{t}_{27}=0.19, \mathrm{p}=0.848$ \\
\hline Mean years disease duration & - & - & $7.54 \pm 5.12$ & $8.33 \pm 5.96$ & $\mathrm{t}_{27}=-0.38, \mathrm{p}=0.850$ \\
\hline Total lesion volume (mL) & - & - & $4.39 \pm 4.88$ & $2.36 \pm 3.56$ & $\mathrm{t}_{27}=1.28, \mathrm{p}=0.210$ \\
\hline $\mathrm{BPF}$ & $0.86 \pm 0.01^{\mathrm{C}, \mathrm{D}}$ & $0.85 \pm 0.02^{\mathrm{C}, \mathrm{D}}$ & $0.84 \pm 0.02$ & $0.84 \pm 0.01$ & $\begin{array}{l}\text { Training: } F_{1,54}=0.188, p=0.666 \\
\text { Group: } F_{1,54}=\mathbf{1 0 . 3 0 1}, \mathbf{p}=\mathbf{0 . 0 0 2} \\
\text { Interaction: } F_{1,54}=0.367, p=0.547\end{array}$ \\
\hline BDI & $7.85 \pm 5.65^{\mathrm{C}, \mathrm{D}}$ & $4.50 \pm 5.24^{\mathrm{C}, \mathrm{D}}$ & $14.21 \pm 7.98$ & $11.47 \pm 8.33$ & $\begin{array}{l}\text { Training: } F_{1,54}=1.844, p=0.181 \\
\text { Group: } F_{\mathbf{1}, \mathbf{5 4}}=\mathbf{8 . 8 3 1}, \mathbf{p}=\mathbf{0 . 0 0 5} \\
\text { Interaction: } F_{1,54}=0.018, p=0.894\end{array}$ \\
\hline FSS & - & - & $47.36 \pm 16.01$ & $40.80 \pm 17.98$ & $\mathrm{t}_{27}=1.03, \mathrm{p}=0.310$ \\
\hline
\end{tabular}

HCu: HC untrained group; HCt: HC trained group; MSu: MS untrained group; MSt: MS trained group; Educational level: $1=$ Primary education, $2=L$ Lower secondary education, 3=Upper secondary education, 4=Post-secondary education non-tertiary, 5=First stage of tertiary education, $6=$ Second stage of tertiary education; EDSS: Expanded Disability Status Scale; BPF: Brain Parenchymal Fraction; BDI: Beck Depression Inventory; FSS: Fatigue Severity Scale. ${ }^{\text {A }}$ denotes statistically significant different from the $\mathrm{HCu}$ group; ${ }^{\mathrm{B}}$ denotes statistically significant different from the $\mathrm{HCt}$ group; ${ }^{\mathrm{C}}$ denotes statistically significant different from the $\mathrm{MSu}$ group; ${ }^{\mathrm{D}}$ denotes statistically significant different from the MSt group. 
Table 1B. Neuropsychological performance data of all participants

\begin{tabular}{|c|c|c|c|c|c|}
\hline Results & $\begin{array}{l}\mathbf{H C}_{\mathbf{U}}(\mathbf{n}=\mathbf{1 5}) \\
(\text { mean } \pm \text { SD })\end{array}$ & $\begin{array}{l}\mathbf{H C}_{\mathbf{T}}(\mathbf{n}=\mathbf{1 4}) \\
(\text { mean } \pm \text { SD })\end{array}$ & $\begin{array}{l}\mathbf{M S}_{\mathbf{U}}(\mathbf{n}=\mathbf{1 4}) \\
(\text { mean } \pm \text { SD })\end{array}$ & $\begin{array}{l}\mathbf{M S}_{\mathbf{T}}(\mathbf{n}=15) \\
(\text { mean } \pm \text { SD })\end{array}$ & Differences \\
\hline \multicolumn{6}{|l|}{ BRN-B } \\
\hline PASAT (\%) & $76.22 \pm 8.87$ & $78.33 \pm 17.45$ & $74.86 \pm 18.18$ & $83.44 \pm 13.01$ & $\begin{array}{l}\text { Training: } F_{1,54}=1.398, p=0.244 \\
\text { Group: } F_{1,54}=0.172, p=0.681 \\
\text { Interaction: } F_{1,54}=0.511, p=0.479\end{array}$ \\
\hline SDMT & $59.69 \pm 9.09$ & $66.17 \pm 6.37$ & $54.93 \pm 10.56$ & $60.80 \pm 10.13$ & $\begin{array}{l}\text { Training: } F_{1,54}=3.813, p=0.056 \\
\text { Group: } F_{1,54}=3.115, p=0.083 \\
\text { Interaction: } F_{1,54}=0.475, p=0.494\end{array}$ \\
\hline SRT Long-Term Storage & $58.46 \pm 8.08$ & $52.67 \pm 12.94$ & $52.07 \pm 13.53$ & $52.53 \pm 10.29$ & $\begin{array}{l}\text { Training: } F_{1,54}=0.598, p=0.444 \\
\text { Group: } F_{1,54}=0.895, p=0.349 \\
\text { Interaction: } F_{1,54}=0.823, p=0.369\end{array}$ \\
\hline SRT Consistent Long-Term Retrieval & $51.31 \pm 11.76$ & $47.83 \pm 5.71$ & $43.21 \pm 14.75$ & $42.07 \pm 12.32$ & $\begin{array}{l}\text { Training: } \mathrm{F}_{1,54}=0.314, \mathrm{p}=0.578 \\
\text { Group: } \mathrm{F}_{1,54}=2.822, \mathrm{p}=0.100 \\
\text { Interaction: } \mathrm{F}_{1,54}=0.080, \mathrm{p}=0.779\end{array}$ \\
\hline SRT Delayed Recall & $10.23 \pm 1.92$ & $10.50 \pm 1.98$ & $9.50 \pm 2.85$ & $10.27 \pm 1.98$ & $\begin{array}{l}\text { Training: } F_{1,54}=0.552, p=0.462 \\
\text { Group: } F_{1,54}=0.478, p=0.493 \\
\text { Interaction: } F_{1,54}=0.127, p=0.723\end{array}$ \\
\hline SPART Long-Term Storage & $20.62 \pm 6.64$ & $23.33 \pm 3.26$ & $20.57 \pm 5.06$ & $20.33 \pm 5.18$ & $\begin{array}{l}\text { Training: } F_{1,54}=0.549, p=0.463 \\
\text { Group: } F_{1,54}=0.827, p=0.368 \\
\text { Interaction: } F_{1,54}=0.780, p=0.382\end{array}$ \\
\hline SPART Delayed-Recall & $7.08 \pm 2.72$ & $8.67 \pm 1.75$ & $7.07 \pm 2.12$ & $7.27 \pm 1.83$ & $\begin{array}{l}\text { Training: } \mathrm{F}_{1,54}=1.748, \mathrm{p}=0.193 \\
\text { Group: } \mathrm{F}_{1,54}=1.084, \mathrm{p}=0.304 \\
\text { Interaction: } \mathrm{F}_{1,54}=1.067, \mathrm{p}=0.307\end{array}$ \\
\hline WLGT & $22.54 \pm 3.57$ & $25.17 \pm 3.66$ & $21.14 \pm 6.29$ & $21.40 \pm 5.58$ & $\begin{array}{l}\text { Training: } \mathrm{F}_{1,54}=0.821, \mathrm{p}=0.370 \\
\text { Group: } \mathrm{F}_{1,54}=2.627, \mathrm{p}=0.112 \\
\text { Interaction: } \mathrm{F}_{1,54}=0.554, \mathrm{p}=0.461\end{array}$ \\
\hline Matrix Subtest (WAIS III) & $105.71 \pm 14.79$ & $106.43 \pm 16.34$ & $111.15 \pm 7.95$ & $106.33 \pm 12.17$ & $\begin{array}{l}\text { Training: } F_{1,54}=0.335, p=0.565 \\
\text { Group: } F_{1,54}=0.567, p=0.455 \\
\text { Interaction: } F_{1,54}=0.608, p=0.439\end{array}$ \\
\hline
\end{tabular}

HCu: untrained group; HCt: HC trained group; MSu: MS untrained group;MSt: MS trained group. BRN-B: The Brief Repeatable Battery of Neuropsychological Test; SDMT: Symbol Digit Modalities Test; PASAT: Paced Auditory Serial Addition Test; SRT: Selective Reminding Test; SPART: Spatial Recall Test; WLGT: Word List Generation Test. 


\section{A. Trained groups < Untrained groups (S2 vs. S1)}
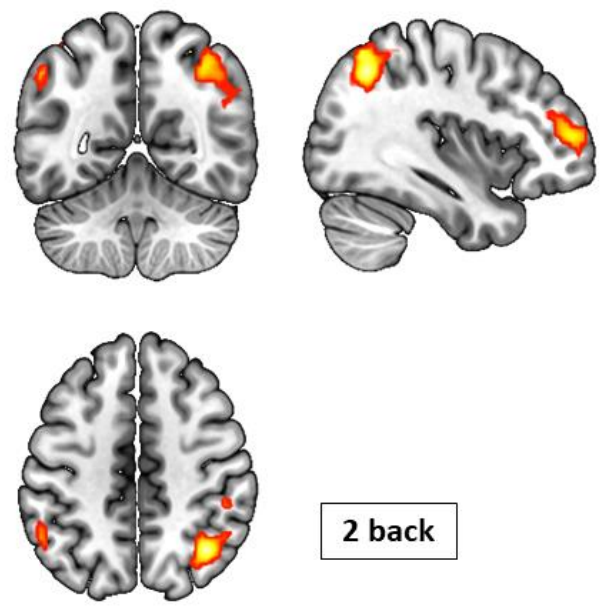

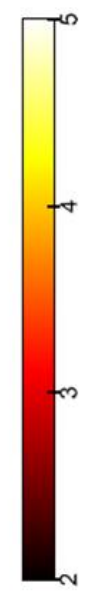

B. Trained groups < Untrained groups (S2 vs. S1)
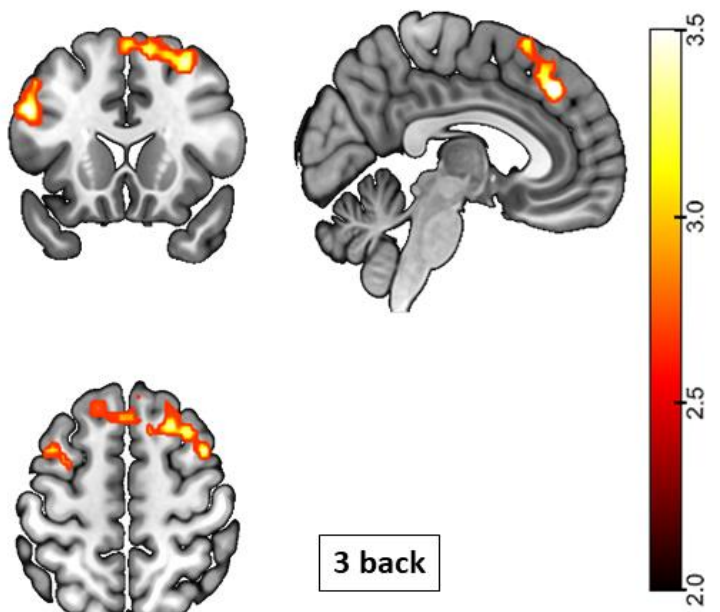

Figure 2. Neuroimaging results. Panel A depicts the decreased activation $(\mathrm{S} 2<\mathrm{S} 1)$ in fronto-parietal areas observed during 2-back performance in trained groups as compared to untrained groups ( $\mathrm{FWEc}=75, \mathrm{p}<0.001)$. Panel B shows the decreased frontoparietal activations $(\mathrm{S} 2<\mathrm{S} 1)$ of trained groups as compared to untrained groups during 3-back performance $(\mathrm{FWEc}=183$, $\mathrm{p}<0.005)$.

\subsection{N-back fMRI task performance}

As Table 3A and Figure 3 show, only the training factor yielded a statistically significant effect on the number of CRs on the 2-back task. Conversely, the RT on the 2-back task was affected by the training effects and session factors as well as their interaction (Table 3B). Post-hoc comparisons revealed a RT decrease in trained (but not in untrained) groups across sessions. Thus, although they had similar RTs in the baseline session, trained groups showed a statistically significant faster performance on the 2-back task than untrained groups in $\mathrm{S} 2$ and $\mathrm{S} 3$.

Both the number of CRs and the RTs on the 3-back task (Table 4 A and B, Figure 3) were mainly dependent on a session $\mathrm{x}$ training condition interaction. Thus, only trained groups exhibited statistically significant increases in CRs and accompanying reductions in RT across sessions. Consequently, although trained groups had similar RTs and a slightly lower number of CRs in the baseline session than the untrained groups, trained groups exhibited shorter RTs and a larger number of CRs in S2 and S3 
Table 2. List of brain activations as a result of the post-training session (S2) in 2-back and 3-back load level between groups comparisons. Results were $p<0.05$ FWEc cluster-corrected using a threshold of $p$ $<0.001$ at the uncorrected level, and a cluster extension of $\mathrm{k}=$ voxels respectively. (MNI: Montreal Neurological Institute coordinates; S1: basal session; S2: follow up session 2.

\begin{tabular}{|c|c|c|c|c|c|}
\hline \multirow[t]{2}{*}{ Anatomical regions } & \multirow[t]{2}{*}{$\mathbf{K}_{\text {voxels }}$} & \multicolumn{3}{|c|}{ MNI coordinates } & \multirow[t]{2}{*}{$\mathbf{T}$} \\
\hline & & $x$ & $y$ & $z$ & \\
\hline \multicolumn{6}{|c|}{ 2-back: Trained groups < Untrained groups (S2vs.S1) } \\
\hline R Angular gyrus & 319 & 36 & -61 & 44 & 5.01 \\
\hline R Supramarginal gyrus & & 48 & -37 & 41 & 3.99 \\
\hline R Inferior parietal lobule & & 48 & -43 & 56 & 3.93 \\
\hline R Middle frontal gyrus & 130 & 39 & 53 & 8 & 4.53 \\
\hline L Inferior parietal lobule & 75 & -54 & -55 & 41 & 4.04 \\
\hline L Postcentral gyrus & & -45 & -37 & 62 & 3.53 \\
\hline \multicolumn{6}{|c|}{ 3-back: Trained groups < Untrained groups (S2vs.S1) } \\
\hline R Superior medial frontal gyrus & 320 & 6 & 35 & 41 & 4.15 \\
\hline R Middle frontal gyrus & & 42 & 8 & 59 & 3.77 \\
\hline R Superior frontal gyrus & & 24 & 23 & 53 & 3.58 \\
\hline L Superior frontal gyrus & & -15 & 29 & 53 & 3.57 \\
\hline R Supplementary motor area & & 9 & 20 & 62 & 3.44 \\
\hline L Supplementary motor area & & 0 & 17 & 62 & 3.25 \\
\hline L Inferior frontal & 183 & -51 & 17 & 29 & 3.82 \\
\hline L Precentral gyrus & & -48 & 11 & 47 & 3.78 \\
\hline L Middle frontal gyrus & & -42 & 8 & 56 & 3.64 \\
\hline L Inferior frontal gyrus & & -45 & 14 & 14 & 2.88 \\
\hline
\end{tabular}


Table 3.A 2-back correct responses. Omnibuses of the ANCOVA (covariates: sex and age) for the number of correct responses during 2-back performance. Descriptive statistics are provided as mean and standard deviation. Statistically significant effects are highlighted in bold.

\begin{tabular}{|c|c|c|c|c|}
\hline \multicolumn{5}{|c|}{ 2-BACK CORRECT RESPONSES } \\
\hline \multicolumn{2}{|l|}{ MAIN EFFECTS } & \multicolumn{3}{|c|}{ DESCRIPTIVE STATISTICS } \\
\hline \multicolumn{5}{|l|}{$\operatorname{Group}\left(\mathrm{F}_{1,52}=0.686, \mathrm{p}=0.41, \eta^{2}=.013\right)$} \\
\hline Training $\left(F_{1,52}=7.12, p<0.01, \eta^{2}=0.12\right)$ & \multirow{3}{*}{$\begin{array}{l}\text { UNTRAINED } \\
\text { Mean } \\
S D\end{array}$} & \multirow{3}{*}{$\begin{array}{l}S 1 \\
14.07 \\
3.53\end{array}$} & \multirow{3}{*}{$\begin{array}{l}S 2 \\
14.76 \\
2.46\end{array}$} & \multirow{3}{*}{$\begin{array}{l}S 3 \\
14.89 \\
3.13\end{array}$} \\
\hline Session $\left(\mathrm{F}_{2,51}=2.85, \mathrm{p}=0.07, \eta^{2}=0.10\right)$ & & & & \\
\hline INTERACTIONS & & & & \\
\hline \multicolumn{5}{|l|}{$\begin{array}{l}\text { Training } x \text { Session } \\
\left(\mathrm{F}_{2,51}=1.88, \mathrm{p}=0.16, \eta^{2}=0.07\right)\end{array}$} \\
\hline Training x Group & TRAINED & $S 1$ & $S 2$ & $S 3$ \\
\hline$\left(\mathrm{F}_{2,51}=0.017, \mathrm{p}=0.90, \eta^{2}=0.00\right)$ & Mean & 14.41 & 16.62 & 16.80 \\
\hline Session x Group & $S D$ & 2.34 & 1.99 & 1.13 \\
\hline \multicolumn{5}{|l|}{$\left(F_{2,51}=0.001, p=0.99, \eta^{2}=0.00\right)$} \\
\hline \multicolumn{5}{|l|}{ Training $\mathrm{x}$ Session $\mathrm{x}$ Group } \\
\hline$\left(\mathrm{F}_{2,51}=0.126, \mathrm{p}=0.88, \eta 2=0.005\right)$ & & & & \\
\hline
\end{tabular}


Table 3.B 2-back reaction times. Omnibuses of the ANCOVAs (covariates: sex and age) for the reaction times during 2-back performance. Within and between comparisons were conducted using Bonferroni post-hoc tests, and their corresponding significance levels (p) and effect sizes (Cohen's d and its 95\% confidence interval) are reported. Statistically significant effects are highlighted in bold. (U, untrained; T, trained).

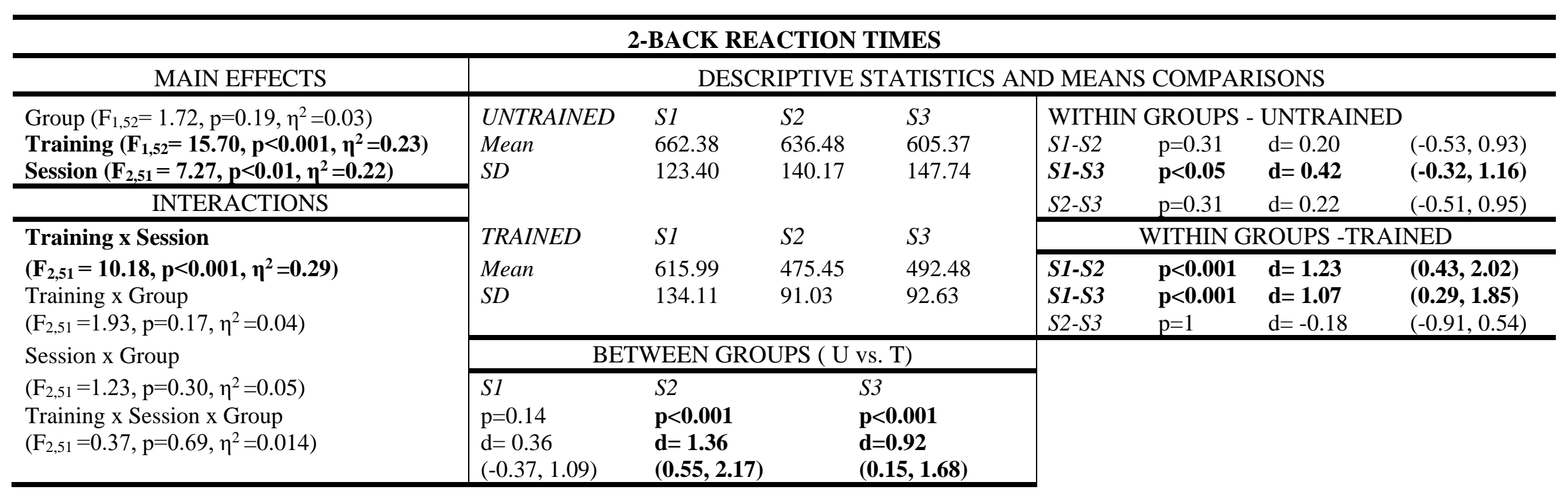


Table 4A. 3-back correct responses. Omnibuses of the ANCOVA (covariates: sex and age) for the number of correct responses during 3-back performance. Within and between comparisons were conducted using Bonferroni post-hoc tests, and their corresponding significance levels (p) and effect sizes (Cohen's $\mathrm{d}$ and its $95 \%$ confidence interval) are reported. Statistically significant effects are highlighted in bold. (U, untrained; T, trained).

\begin{tabular}{|c|c|c|c|c|c|c|c|c|}
\hline \multicolumn{9}{|c|}{ 3-BACK CORRECT RESPONSES } \\
\hline MAIN EFFECTS & \multicolumn{8}{|c|}{ DESCRIPTIVE STATISTICS AND MEANS COMPARISONS } \\
\hline $\operatorname{Group}\left(\mathrm{F}_{1,52}=0.72, \mathrm{p}=0.40, \eta^{2}=0.014\right)$ & UNTRAINED & $S 1$ & $S 2$ & $S 3$ & \multicolumn{4}{|c|}{ WITHIN GROUPS - UNTRAINED } \\
\hline $\begin{array}{l}\text { Training }\left(F_{1,52}=2.78, p=0.10, \eta^{2}=0.51\right) \\
\text { Session }\left(F_{2,51}=1.18, p=0.31, \eta^{2}=0.04\right)\end{array}$ & $\begin{array}{l}\text { Mean } \\
S D\end{array}$ & $\begin{array}{l}13.14 \\
3.62\end{array}$ & $\begin{array}{l}12.93 \\
3.52\end{array}$ & $\begin{array}{l}14.06 \\
4.12\end{array}$ & $\begin{array}{l}S 1-S 2 \\
S 1-S 3\end{array}$ & $\begin{array}{l}\mathrm{p}=1.00 \\
\mathrm{p}=0.72\end{array}$ & $\begin{array}{l}\mathrm{d}=0.06 \\
\mathrm{~d}=-0.24\end{array}$ & $\begin{array}{l}(-0.67,0.79) \\
(-0.97,0.49)\end{array}$ \\
\hline INTERACTIONS & & & & & $S 2-S 3$ & $\mathrm{p}=0.072$ & $\mathrm{~d}=-0.29$ & $(-1.03,0.44)$ \\
\hline Training x Session & TRAINED & S1 & $S 2$ & S3 & \multicolumn{4}{|c|}{ WITHIN GROUPS - TRAINED } \\
\hline $\begin{array}{l}\left(\mathbf{F}_{\mathbf{2 , 5 1}}=\mathbf{1 9 . 8 1}, \mathbf{p}<\mathbf{0 . 0 0 1}, \mathbf{\eta}^{\mathbf{2}}=\mathbf{0 . 4 4}\right) \\
\text { Training x Group } \\
\left(\mathrm{F}_{1,52}=0.22, \mathrm{p}=0.64, \eta^{2}=0.004\right)\end{array}$ & $\begin{array}{l}\text { Mean } \\
S D\end{array}$ & $\begin{array}{l}11.10 \\
4.08\end{array}$ & $\begin{array}{l}16.41 \\
1.59\end{array}$ & $\begin{array}{l}16.47 \\
1.56\end{array}$ & $\begin{array}{l}S 1-S 2 \\
S 1-S 3 \\
S 2-S 3\end{array}$ & $\begin{array}{l}p<0.001 \\
p<0.001 \\
p=1.00\end{array}$ & $\begin{array}{l}d=-1.71 \\
d=-1.74 \\
d=-0.04\end{array}$ & $\begin{array}{l}(-2.57,0.86) \\
(-2.59,0.88) \\
(-0.77,0.69) \\
\end{array}$ \\
\hline Session x Group & \multicolumn{4}{|c|}{ BETWEEN GROUPS (U vs. T) } & & & & \\
\hline $\begin{array}{l}\left(\mathrm{F}_{2,51}=0.20, \mathrm{p}=0.82, \eta^{2}=0.008\right) \\
\text { Training x Session x Group } \\
\left(\mathrm{F}_{2,51}=1.62, \mathrm{p}=0.21, \eta^{2}=0.06\right)\end{array}$ & $\begin{array}{l}S 1 \\
\mathbf{p}=\mathbf{0 . 0 3 5} \\
\mathbf{d}=\mathbf{0 . 5 3} \\
(\mathbf{- 0 . 2 1 , 1 . 2 7 )}\end{array}$ & $\begin{array}{l}S 2 \\
p<0.001 \\
d=-\mathbf{1 . 2 7} \\
(-\mathbf{2 . 0 7}, \mathbf{- 0 . 4 8 )}\end{array}$ & & $\begin{array}{l}S 3 \\
\mathbf{p}<0.001 \\
d=-\mathbf{0 . 7 7} \\
(-\mathbf{1 . 5 3 , - 0 . 0 2 )}\end{array}$ & & & & \\
\hline
\end{tabular}


Table 4B. 3-back reaction times. Omnibuses of the ANCOVA (covariates: sex and age) for the reaction times during 3-back performance. Within and between comparisons were conducted using Bonferroni post-hoc tests, and their corresponding significance levels (p) and effect sizes (Cohen's d and its 95\% confidence interval) are reported. Statistically significant effects are highlighted in bold. (U, untrained; T, trained).

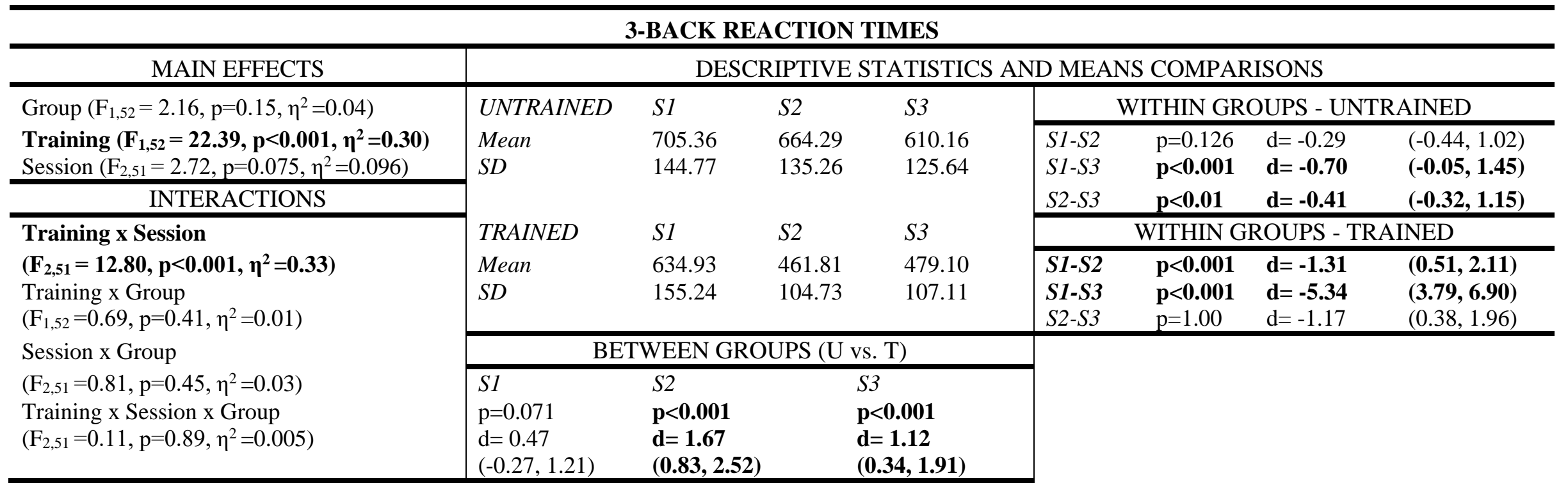



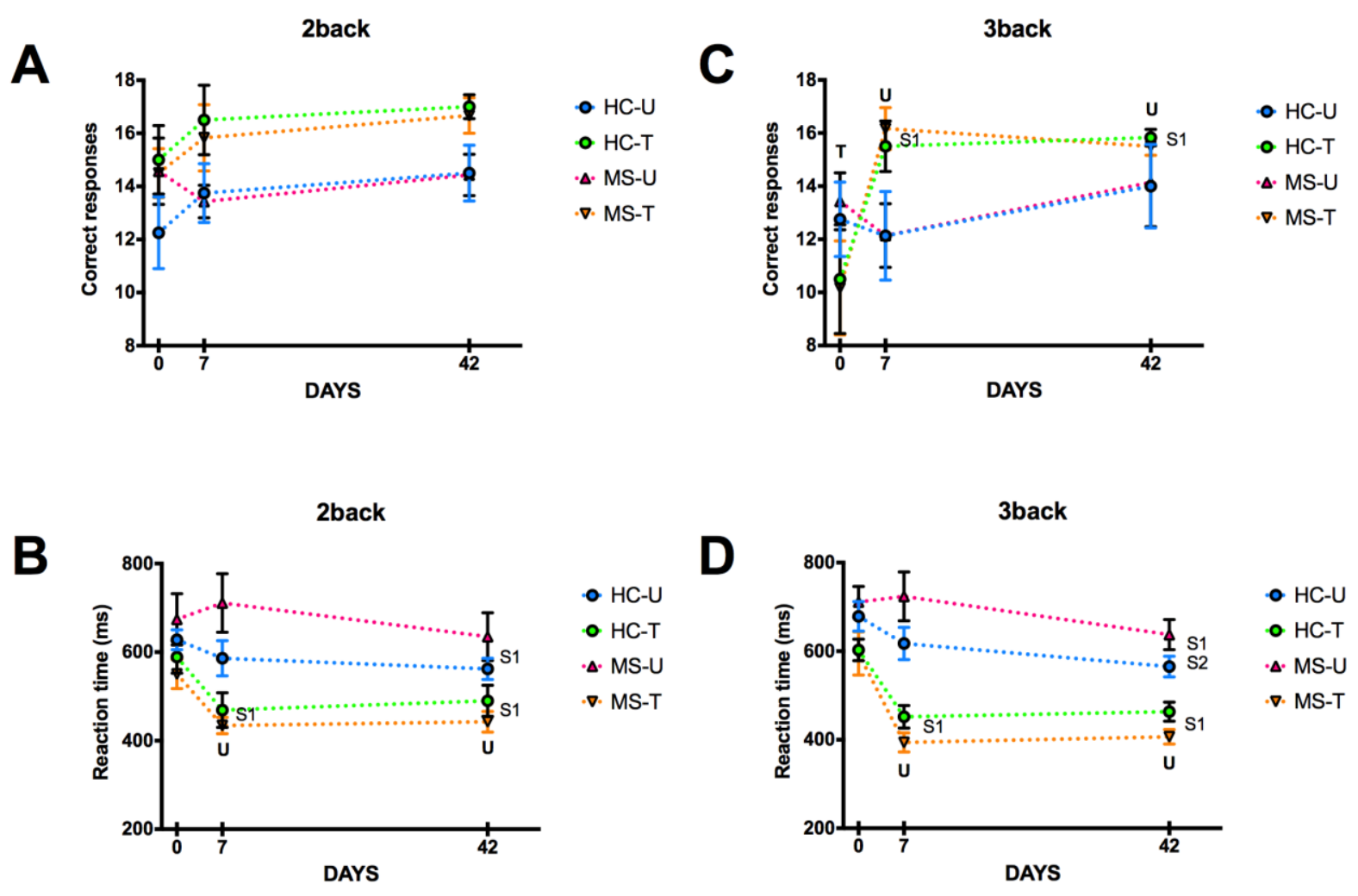

Figure 3. Behavioral results. Panels depict mean \pm SEM of correct responses (CRs) and reaction times (RTs) in the 2-back and 3-back tasks at each session. Trained groups exhibited shorter RTs but not an increased number of CRs (panel A) in the 2-back task at the post-training (S2) and in the follow-up (S3) sessions than in the baseline (S1) session. Trained groups exhibited higher number of CRs (panel C) and shorter RTs (panel D) in the 3-back task at S2 and S3 than in S1. (U, different from untrained groups; T, different from trained groups; $\mathrm{S} 1$, different from baseline; S2, different from post-training session; see tables 3 and 4 for details).

\subsection{N-back training performance test}

The 2-back repeated measures ANOVA revealed a significant effect on CRs of training sessions $\left(\mathrm{F}_{3,81}=3.86, \mathrm{p}<0.05, \eta^{2}=0.114\right)$, but not group $\left(\mathrm{F}_{1,27}=0.046, \mathrm{p}=0.831, \eta^{2}=0.002\right)$ or the first-order interaction $\left(\mathrm{F}_{3,81}=1.32, \mathrm{p}=0.273, \eta^{2}=0.047\right)$. Paired t-tests showed an increased number of CRs in $\mathrm{T} 4$ than in $\mathrm{T} 1(\mathrm{t}=2.479, \mathrm{p}<0.05)$.

In the same way, the 2-back repeated measures ANOVA revealed a significant effect on RTs of training sessions $\left(\mathrm{F}_{3,81}=10.72, \mathrm{p}<0.001, \mathrm{\eta}^{2}=0.284\right)$, but not group $\left(\mathrm{F}_{1,27}=0.022, \mathrm{p}=0.883, \mathrm{\eta}^{2}=0.001\right)$ or the first-order interaction $\left(\mathrm{F}_{3,81}=1.04, \mathrm{p}=0.378, \mathrm{n}^{2}=0.037\right)$. Paired t-tests showed shorter RTs in $\mathrm{T} 4$ than in $\mathrm{T} 1(\mathrm{t}=-4.33, \mathrm{p}<0.001)$. 
The 3-back repeated measures ANOVA revealed a significant effect on CRs of training sessions $\left(F_{3,81}=10.05, p<0.001, \eta^{2}=0.271\right)$, but not group $\left(F_{1,27}=0.087, p=0.770, \eta^{2}=0.003\right)$ or the first-order interaction $\left(\mathrm{F}_{3,81}=0.746, \mathrm{p}=0.528, \eta^{2}=0.027\right)$. Paired $\mathrm{t}$-tests showed an increased number of $\mathrm{CRs}$ in $\mathrm{T} 4$ than in $\mathrm{T} 1(\mathrm{t}=3.89, \mathrm{p}<0.001)$.

Similarly, the 3-back repeated measures ANOVA revealed a significant effect on RTs of training sessions $\left(\mathrm{F}_{3,81}=7.15, \mathrm{p}<0.001, \mathrm{\eta}^{2}=0.209\right)$, but not group $\left(\mathrm{F}_{1,27}=0.270, \mathrm{p}=0.608, \eta^{2}=0.010\right)$ or the firstorder interaction $\left(\mathrm{F}_{3,81}=1.04, \mathrm{p}=0.377, \mathrm{n}^{2}=0.037\right)$. Paired t-tests showed shorter $\mathrm{RT}$ s in $\mathrm{T} 4$ than in $\mathrm{T} 1$ $(\mathrm{t}=-3.48, \mathrm{p}<0.01)$.

\subsection{Relationship between the effects of training on cerebral activation and cognitive performance}

The S2 vs S1 decrease in activation in the right medial frontal gyrus during 2-back performance was significantly correlated with the corresponding RT decreases between these sessions ( $\mathrm{r}=0.346$, $\mathrm{p}<0.01)$. Moreover, the $\mathrm{S} 2 \mathrm{vs} \mathrm{S} 1$ decrease in activation focused on the right superior frontal gyrus correlated with the RT reduction during the performance of the 3-back task in these sessions $(\mathrm{r}=0.372$, $\mathrm{p}<0.01$ ). Finally, despite a significant reduction in the number of data due to technical problems (see methods section), a large and direct correlation $(r=0.492, p<0.05)$ between the $S 3$ vs $S 1$ decrease in activation in the right superior frontal gyrus was observed during 2-back performance.

\section{Discussion}

The main outcome of this randomized controlled study was the confirmation of the efficacy of a short but intensive cognitive training program in $\mathrm{HC}$ and MS patients. More specifically, after four days (60 min/ day) of WMF training, both MS patients and HC showed a significant improvement in their performance on the WMF trained task. Moreover, these performance improvements were accompanied by a significant decrease in brain activity in some fronto-parietal areas belonging to the WMF network. These results suggest that this training program increases cognitive and neural efficiency.

Thus, compared to the non-trained groups of MS patients and HC, trained (HC and MS patients) participants exhibited lower S2 RTs and an increase in CRs during the performance of the 2- and 3back tasks. In addition, during the performance of the 2-back task, trained participants showed decreased activation in bilateral fronto-parietal areas, which belong to the previously described WMF network (Miró-Padilla et al., 2018; Owen et al., 2005). A similar reduction in brain activity, although

in this case restricted to bilateral frontal areas of the same network, was observed during the 
performance of the 3-back task. Interestingly, the beneficial effects of this training program were persistent over time. Thus, more than 42 days after the end of S3, WMF performance in trained participants remained as good as immediately after completing the training program (S2), and it was significantly higher than before its implementation (S1). In S3, enhanced performance was again accompanied by a significant brain activity reduction in right fronto-parietal areas. These findings are consistent with those of a similar study that reported improved performance and decreased activation in frontal and parietal areas after n-back training in young healthy volunteers (Miró-Padilla et al., 2018) and they might be interpreted in the context of the "neural efficiency hypothesis" (Buschkuehl et al., 2012; Miró-Padilla et al., 2018).

The concept of neural efficiency refers to achieving maximum performance levels while deploying a minimum amount of brain resources, and it has received experimental support from previous studies involving healthy volunteers (Buschkuehl et al., 2012; Forn et al., 2013; Miró-Padilla et al., 2018) and MS patients (Fittipaldi-Márquez et al., 2017). Thus, because in our study WMF training resulted in increased performance and reduced activation of the fronto-parietal WMF network, it might be concluded that this training program in- creased both cognitive performance and neural efficiency. Direct support for this interpretation is provided by the observed statistically significant correlations between the reduction in the activation in frontal areas and the reduction in the RTs during the performance of 2 and 3-back tasks. In particular, we observed that the S2 vs S1 reduction in right medial frontal gyrus activity is strongly related to the S2 vs S1 decline in RTs on the 2-back tasks. A similar correlation was observed between the S2 vs S1 activity reduction in the right superior frontal gyrus and in the RTs observed during the performance of the 3-back task. Moreover, despite a significant reduction in the number of data due to technical problems (see methods section), a large and direct correlation $(0.492, \mathrm{p}<0.05)$ between the S3 vs S1 decrease in activation in the right precentral gyrus and the S3 vs. S1 reduction in the 3-back RTs was observed. Taken together, these results confirm that the cognitive training program used in the present study enhances both performance on the n-back task and neural efficiency.

However, it should be noted that our results and their interpretation seem to be at odds with those of other previous studies exploring neuroplasticity changes in response to cognitive rehabilitation in MS patients (Campbell et al., 2016; Cerasa et al., 2013; Chiaravalloti et al., 2013; Ernst, Sourty, Roquet, Noblet, Gounot, Blanc, De Seze, et al., 2016; Filippi, Riccitelli, et al., 2012; Sastre-Garriga et al., 2010). These studies have generally observed an improvement in cognitive performance, but at the cost of a higher degree of brain activation, that is, the exact opposite of what we observed in the present study. This discrepancy seems to be due to the distinct clinical status of the MS patients in 
each case. Indeed, when this variable and the inverted-U relationship between brain activation and cognitive performance observed throughout MS progression (Penner et al., 2006, 2007) are taken into account, all these results seem to conform a coherent picture. Thus, it should be noted that we recruited a homogeneous group of MS patients with the same clinical phenotype (RRMS) and no cognitive impairment, and even more importantly, we chose MS patients who did not achieve this normal cognitive performance through the early spontaneous adaptive neuroplasticity processes (e.g. increased brain activation) described in previous studies (Fittipaldi-Márquez et al., 2017; Forn et al., 2006, 2007). Therefore, the patients recruited in our study were very similar to HC, and the expected effects of cognitive training should also be the same as in HC, namely, an increase in performance and neural efficiency (Fittipaldi-Márquez et al., 2017; Miró-Padilla et al., 2018; Penner et al., 2007) moderate cognitive impairment. In these mildly impaired MS patients, increased cognitive performance is expected to be achieved only if additional brain resources can be recruited (Chiaravalloti et al., 2005; Fittipaldi-Márquez et al., 2017; Penner et al., 2007), which is exactly what was observed after they received neuropsychological training (Campbell et al., 2016; Cerasa et al., 2013; Chiaravalloti et al., 2013; Ernst et al., 2013; Filippi, Riccitelli, et al., 2012; Sastre-Garriga et al., 2010).

On the other hand, it should also be noted that our study differs from others (Campbell et al., 2016; Cerasa et al., 2013; Chiaravalloti et al., 2013; Ernst, Sourty, Roquet, Noblet, Gounot, Blanc, De Seze, et al., 2016; Filippi, Riccitelli, et al., 2012; Sastre-Garriga et al., 2010) in another aspect. Whereas our training program was designed to improve a specific cognitive domain (WMF), previous studies tried to improve several cognitive functions at the same time. However, the possible relevance of this procedural difference, if any, when trying to explain the distinct dynamics of brain activation after cognitive training, remains unknown. By contrast, it might safely be concluded that intensive training using the n-back task, which involves information maintenance and manipulation, but also a substantial information processing speed demand, results in improved WMF performance, enhanced information processing speed (as revealed by RT reduction), and consistent cerebral changes in the WMF fronto-parietal network.

Finally, the present study also presents some limitations that should be pointed out. First, as in most of the preceding studies exploring the beneficial effects of cognitive training in MS patients (Mitolo et al., 2015; Prosperini et al., 2015), the present results were obtained in a small sample of participants, hence reducing the statistical power of our study and the generalizability of its results. The small sample problem was further aggravated by the loss of a significant proportion of participants' data from S3. Second, as discussed above, the results of the present study were obtained with a carefully 
selected and homogeneous group of MS patients without cognitive impairment. Third, in this study we used a repeated and intensive WMF training that is not directly comparable to previous studies using other rehabilitation programs aimed to improve a wide range of cognitive functions. Therefore, these results should not be over-generalized, and further studies are needed to determine whether this specific WMF training program is also useful for improving other cognitive skills (performance transfer effects) in MS patients with cognitive impairment and apparent brain damage (Buschkuehl et al., 2012; Mitolo et al., 2015). Finally, we did not assess this possible generalization effect to other cognitive functions and daily life activities, which are important issues that should be explored in future studies with a large sample of MS patients.

\section{Conclusions}

In summary, the results of this study show that an intensive WMF training program enhances performance and decreases the cerebral activation of WMF networks in MS and HC patients. These findings are promising and warrant further studies assessing the effects of this training in larger cohorts of MS patients with different degrees of cognitive impairment.

\section{Data Availability}

The behavioral and imaging data corresponding to the findings of the present study are available from the corresponding author upon request.

\section{Conflicts of Interest}

The authors declared no potential conflicts of interest with respect to the research, authorship and the publication of this article.

\section{Acknowledgements}

This research was supported by a grant (PSI2015-67285-R) provided by Ministerio de Educación, Cultura y Deporte to CF and by a grant (UJI B2017-05) awarded to CS-S. NA was supported by a FPU grant from the Spanish Ministry of Education (FPU16/01525)

\section{Supplementary material}

Supplementary Table 1. Mean and standard deviations (mean \pm SD) of performance in tests of each training session (TS).

Supplementary Table 2: List of brain activations as a result of the post-training session (S3) in 2back load level between groups comparisons. 
Supplementary Figure 1. N-back training protocol.

Supplementary Figure 2. Activations in fronto-parietal areas for healthy controls (HC) and multiple sclerosis (MS) patients during WMF performance

Supplementary Figure 3. Neuroimaging results as a result of the post-training session (S3), compared to the basal session (S1), in 2-back load level between-group comparisons.

Supplementary Figure 4. Activity changes in fronto-parietal areas during WMF performance. 


\section{Study III}

Enhanced frontoparietal connectivity in multiple sclerosis patients and healthy controls in response to an intensive computerized training focused on working memory

Naiara Aguirre ${ }^{1}$, Álvaro Javier Cruz-Gómez ${ }^{2}$, Sonia Félix Esbrí ${ }^{1}$, Anna Miró-Padilla ${ }^{1}$, Elisenda Bueichekú ${ }^{1}$, Ricardo Broseta-Torres ${ }^{3}$, César Ávila ${ }^{1}$, Carla Sanchis-Segura ${ }^{1}$ and Cristina Forn ${ }^{1}$

${ }^{1}$ Jaume I University. Department of Basic, Clinic and Psychobiology. Neuropsychology and Functional Neuroimaging group

${ }^{2}$ INiBICA. Instituto de Investigación e Innovación Biomédica de Cádiz

${ }^{3}$ ERESA, medic group. Valencia, Spain

doi: 10.1016/j.msard.2021.102976 


\section{Abstract}

Background: Working memory (WMF) deficits are common in multiple sclerosis (MS) patients. Computerized cognitive training may enhance WMF capabilities but its efficacy in MS patients has not been sufficiently explored.

Methods: This study examines the effects of n-back training on cognitive performance and functional connectivity (FC) in 29 MS patients and 29 healthy controls (HC). Baseline (S1) performance on 2and 3-back tasks and FC within the fronto-parietal network were assessed before randomly splitting the sample into four subgroups: trained MS (MSt, n=15), trained HC ( $\mathrm{HCt}, \mathrm{n}=14)$, untrained MS $(\mathrm{MSu}, \mathrm{n}=14)$, and untrained $\mathrm{HC}(\mathrm{HCu}, \mathrm{n}=15)$. The trained subgroups underwent adaptive $\mathrm{n}$-back training (60 min/day; 4 days) and n-back task performance and FC were reassessed in a second session (S2).

Results: As revealed by mixed two-way ANOVAs, trained participants (MSt and HCt) exhibited a significant increase in the number of correct responses and significantly reduced reaction times in $\mathrm{S} 2$. These performance improvements were accompanied by an increase in FC in the fronto-parietal pathways and statistically significant correlations between both effects were found.

Conclusions: Computerised WMF training results in behavioural and neuroplasticity positive effects that may be useful when trying to prevent or attenuate cognitive decline in MS patients.

Key Words: multiple sclerosis, working memory, cognitive training, functional connectivity, n-back. 


\section{Introduction}

There is growing interest in identifying clinical interventions that could delay or reduce the cognitive deficits in multiple sclerosis (MS) patients. In this regard, a recent meta-analysis has pointed out that computer-based cognitive training programs appear to improve cognitive performance in MS patients (Lampit et al., 2019). As the same meta-analysis has concluded (Lampit et al., 2019), more and wellvalidated studies are needed to confirm the usefulness of this kind of interventions in preventing or mitigating the cognitive decline observed in MS patients.

In principle, the efficiency of any training/rehabilitation program can be measured either by observing an improvement in the trained cognitive skills or by exploring the brain's response (neuroplasticity) to rehabilitation programs. However, the effectiveness of cognitive rehabilitation programs only becomes definitively supported when these two kinds of data are linked, producing a real gain in the knowledge about the effects of MS on cognitive competence (Mitolo et al., 2015). On this subject, the neuroplasticity processes induced by cognitive rehabilitation in MS patients have been studied in terms of activation or functional connectivity (FC) changes. Regarding the former, several (but not all) studies have described brain activation increases after cognitive rehabilitation, which in some (but not all) cases were directly correlated with observed improvements in cognitive performance (Chiaravalloti et al., 2012; Ernst et al., 2012; Sastre-Garriga et al., 2010). In the latter case, FC studies present more homogenous results, suggesting that cognitive rehabilitation enhances FC in MS patients (Bonavita et al., 2015; De Giglio, Tona, et al., 2016; Leavitt, Wylie, Girgis, et al., 2014; Parisi, Rocca, Mattioli, et al., 2014; Parisi, Rocca, Valsasina, et al., 2014).

Although the results of these studies are promising, as a recent review (Mitolo et al., 2015) indicate, they are also inconclusive, perhaps due to the heterogeneity of the selected participants, the diversity and lack of specificity of the rehabilitation approaches used, and other methodological weaknesses (e.g., the selection of outcome measures). Therefore, more studies are needed in this area. Moreover, according to the most recent recommendations (Lampit et al., 2019; Mitolo et al., 2015), this research should assess interventions that are implemented through computerised programs, focus on repeated and controlled practice in very structured sessions, and target just one or a few specific cognitive functions. Regarding the latter, behavioural interventions should be designed to boost basic cognitive processes and functions (e.g. working memory, WMF) that subserve higher cognitive abilities and may indirectly promote a generalized enhancement of cognitive performance (Mitolo et al., 2015).

In this respect, previous studies using n-back training task (Covey et al., 2018; Hancock et al., 2015) have described the benefits of specifically implementing this task, not only for WMF but also for 
information processing speed (IPS). We recently conducted an activation-based study to assess the effects of an intensive and adaptive computerised n-back training on the WMF capabilities of MS patients (Aguirre, Cruz-Gómez, et al., 2019). We focused our study on WMF because: 1) about 27$44 \%$ of MS patients show WMF impairments and these impairments affect other cognitive domains, such as IPS, attention, learning capacity and executive functions (Chiaravalloti \& DeLuca, 2008); 2), only a few studies had previously examined changes in cognitive performance and brain activity following WMF training in this clinical population (Covey et al., 2018). Our results revealed that, compared to untrained participants, trained MS and healthy controls (HC) exhibited enhanced 2-back and 3-back task performance, an effect that was correlated with activation changes. Furthermore, we observed that the training-induced improvements in WMF were accompanied by improvements in IPS. These findings suggest that MS patients are able to benefit from computerized WMF training programs capable of promoting neuroplastic changes within the WMF network and that such training program results in enhanced WMF and IPS capabilities, two cognitive domains characteristically affected in MS patients (Covey et al., 2018; DeLuca et al., 2004; Forn et al., 2008; Hancock et al., 2015).

In the present study, we sought to confirm and extend these results by investigating whether the effects of this particular WMF training program on improving n-back performance is also supported by changes in FC. We focus our intervention in MS patients with no cognitive impairment to evaluate if once MS has been already diagnosed but there are no overt signs of cognitive decline, MS patients still retain the same capabilities and potentialities than $\mathrm{HC}$ and equally benefit of cognitive training and repeated practice.

We hypothesized that both trained groups (MS and HC) would present similar increases in FC within some nodes of the WMF network, and that these changes would be correlated with an increase in the number of correct responses (CRs) and a reduction in the reaction times (RTs) on the 2- and 3-back tasks. 


\section{Materials and Methods}

\subsection{Participants}

Right-handed patients with no cognitive impairments and diagnosed with definitive RRMS, according to McDonald criteria, were selected for the study and neurologically assessed using the Expanded Disability Status Scale (EDSS). In order to be included in the study, MS patients had to be free from steroids' treatment and have not experienced any relapse episode in the last 2 months. Patients should not present any other concomitant Central Nervous System pathology or major visual or eye-hand coordination limitations. Moreover, right-handed participants with no neurological or psychiatric dysfunctions made up the control group (HC). Participants (HC and MS) were randomly allocated in different subgroups: MS untrained group ( $\mathrm{MSu}, \mathrm{n}=14)$, $\mathrm{HC}$ untrained group $(\mathrm{HCu}, \mathrm{n}=15)$, MS trained group (MSt, $n=15)$, and $\mathrm{HC}$ trained group ( $\mathrm{HCt}, \mathrm{n}=14)$. All participants gave informed written consent prior to participation and received remuneration for completing the study. The Ethical Committee of Universitat Jaume I approved the research project and was conducted in accordance with the Declaration of Helsinki.

As we describe in a previous study (Forn et al., 2006), participants were neuropsychologically assessed between 5/7 days prior to the scanner with the following measures: 1) Brief Repeatable Battery of Neuropsychological Tests (BRB-N) validated for the Spanish population; Matrix Reasoning Subtest of the Wechsler Adult Intelligence Scale (WAIS III) to assess the intelligence quotient (IQ); Fatigue Severity Scale (FSS); and Beck Depression Inventory (BDI). Moreover, assessment also included two functional Magnetic Resonance Imaging (fMRI) sessions: baseline session (S1) and post-training session (S2; 7 days later).

\subsection{MRI acquisition}

Neuroimaging data were acquired on a 1.5T scanner (Siemens Symphony, Erlangen, Germany) in S1 and S2 in this order: 1) Anatomical 3D MPRAGE volumes were acquired, using a T1-weighted gradient echo pulse sequence $\left(\mathrm{TR}=2200 \mathrm{~ms} ; \mathrm{TE}=3 \mathrm{~ms}\right.$; flip angle $=15^{\circ}$; matrix $=256 \times 256 \times 160$; voxel $=1 \times 1 \times 1 \mathrm{~mm})$, and for MS patients, a FLAIR sequence $(\mathrm{TR}=6000 \mathrm{~ms}$; TE $=354 \mathrm{~ms}$; flip angle $=180^{\circ}$; matrix $=196 \times 256 \times 160$; voxel $=1.05 \times 1.05 \times 1 \mathrm{~mm}$ ); 2) During n-back fMRI were acquired with a gradient-echo $\mathrm{T}^{*}$-weighted echo-planar MR sequence covering the entire brain (TR=2500ms; $\mathrm{TE}=49 \mathrm{~ms}$; matrix $=64 \times 64 \times 28$; flip angle $=90^{\circ}$; voxel $=3.5 \mathrm{~mm}^{3}$; slice gap $=4.41 \mathrm{~mm}$ ). A total of 260 volumes were recorded (Aguirre, Cruz-Gómez, et al., 2019). 
The n-back adapted for fMRI studies is described in Aguirre et al. (2019). Inside the scanner, visual stimuli were presented electronically using E-Prime software (Psychology Software Tools, Pittsburgh, PA), professional version 2.0, installed in a Hewlett-Packard portable workstation (screenresolution 800 x 600, refresh rate of $60 \mathrm{~Hz}$ ). Participants watched the laptop screen through MRIcompatible goggles (VisuaStim, Resonance Technology, Inc., Northridge, CA, USA). During the task, participants had to give "yes" or "no" motor responses that were collected via MRI-compatible response-grips (NordicNeuroLab, Bergen, Norway). The E-Prime's logfile saved the CRs and RTs for each stimulus for each participant.

\section{$2.3 \mathrm{~N}$-back training protocol}

Two days after S1, the trained groups came to the university to complete four WMF training sessions on consecutive days. Each training sessions had a total duration of 60 minutes and they were distributed in two phases. During the first phase, participants performed WMF training, adapted from Jaeggi et al. (2008), for 50 minutes. In this phase, participants performed three runs, each composed of eight blocks that varied in WMF load (1-back, 2-back, and 3-back). For motivational reasons, the training always started at the low level (1-back load), and the level of n-back of the subsequent block was based on the participant's performance on the previous block. Thus, if the participant had at least 90\% CRs, the WMF load increased one level (e.g. 90\% performance on 2-back tasks increased to 3back). If the CRs during the block were below $80 \%$, in the subsequent block the WMF load decreased one level (e.g. from 2-back to 1-back). In all other cases, the n-level remained constant. Participants were instructed to give manual responses only with their right hand, responding to targets with their thumb and to non-targets with their forefinger. Feedback was introduced after each response for a few seconds, as a coloured circle at the corner of the screen: green meant a correct answer, a red circle represented an error, and blue indicated missing responses. Moreover, at the end of each block, subjects also received additional information about their percentage of correct responses (CRs) and the average reaction time (RT) of their responses. Finally, participants completed a test phase that consisted of eight blocks of the 2- and 3-back tasks. Subjects received no feedback during this time. Their results on this test were used to evaluate their progress on n-back execution. For more information, see also Aguirre et al. (2019). 


\subsection{Neuroimaging analysis}

To define regions of interest (ROIs), we extracted the Talairach coordinates of the specific brain regions related to n-back performance reported in Wang et al. (2019) (see also Figure 1 and Supplementary Table 1), and we converted them to the MNI space using the Mango v4.1 toolbox. After that, we used AAL (Tzourio-Mazoyer et al., 2002) to label the corresponding MNI coordinates. Finally, we localized peak coordinates of each AAL region related to the n-back task in our data, and we defined ROIs as 5mm spheres using the WFU-PickAtlas toolbox (Maldjian et al., 2003). Data preprocessing and first-level analysis were performed using the CONN-Toolbox v18.4 (WhitfieldGabrieli \& Nieto-Castanon, 2012) for SPM12, implemented in Matlab 2018b. Anatomical and functional images were preprocessed using the default pipeline implemented in the CONN-Toolbox, including realignment, co-registration, spatial normalization to MNI templates, smoothing of $4 \mathrm{~mm}$ FWHM, and temporal filtering $(0.01 \mathrm{~Hz}-0.08 \mathrm{~Hz})$. In order to study FC during the 2- and 3-back tasks, we performed a ROI-to-ROI analysis using the implemented generalized Psychophysiological Interaction procedure (gPPI). A separate multiple regression model was computed for each target voxel BOLD timeseries. Each model included three independent variables: 1) the main psychological factor, in this study it corresponds to the three task conditions effects (0-back, 2-back and 3-back) convolved with a canonical hemodynamic response function; 2) the main physiological factor, which correspond with each seed ROI BOLD timeseries; and 3) the interaction term specified as the product of the previous two factors. Finally, we extracted the first-level ROI-to-ROI connectivity matrices of each n-back condition (0-back, 2-back and 3-back) and contrast matrices were calculated to test the effects of the task conditions (2-back and 3-back) compared to control condition (0-back).

\section{Statistical analyses}

Second level statistical analyses were conducted with the rstatix package 20 (Kassambara, 2020) for RStudio (version 1.2.5, RStudio, Inc). More specifically, two-way (Group: HC vs. MS; Training: Untrained vs. Trained) ANOVAs were used to compare the groups of participants on the demographic and clinical variables displayed in Table 1. Female/male proportions in these groups were compared by means of the chi-squared test. N-back performance (CRs and RT) and FC scores were analyzed using mixed two-way ANOVAs (group x training x session). Results were considered statistically significant when FWER-corrected $p$-values were below the 0.05 threshold. In these cases, appropriate univariate effect size indexes ( $\eta^{2}$ or Cohen's $d$ ) were calculated. The size of the multivariate betweengroup differences in the degree of change in FC scores $\left(\Delta\right.$-FC scores $\left.=F_{S 2}-F_{S 1}\right)$ during 2- or 3back performance was also estimated. More specifically, the unbiased Mahalanobis' D, the overlap coefficient, and the probability of superiority (PS) were calculated with the maha function (Del 
Giudice et al., 2012), whereas the classification accuracy (percent of correctly classified cases) was calculated with the lda function of the MASS package (Venables \& Ripley, 2002) for RStudio.

In addition, Pearson's $r$ correlation index was used to assess the strength of the relationship between the degree of change in FC scores $(\Delta$-FC scores) and the degree of change in the number of CRs or RT ( $\Delta$-CRs and $\Delta$-TR, respectively). The amount of variance in $\Delta$-CRs or $\Delta$-TR attributable to the collective changes in $\Delta$-FC was estimated in terms of $\mathrm{R}^{2}$ adj and deviance explained, calculated in appropriate General Additive Models with the gam function of the $m g c v$ package for Rstudio (Wood, 2004). The resulting effect sizes were characterized as "small", "medium", or "large", according to the benchmarks proposed by Cohen (1988) for $\mathrm{R}^{2}$ estimates.

\section{Results}

\subsection{Demographic and neuropsychological variables.}

Table 1 displays the demographic and clinical characteristics of each group of participants. Statistically significant differences between groups were only observed for BDI scores and, as expected, for BPF volume. Conversely, these four groups did not significantly differ on age, gender, neuropsychological performance, or fatigue scores.

Table 1. Demographic, clinical and neuropsychological data of all participants

\begin{tabular}{|c|c|c|c|c|c|}
\hline & $\begin{array}{l}\mathbf{H C}_{\mathbf{U}}(\mathbf{n}=\mathbf{1 5}) \\
(\mathrm{mean} \pm \mathrm{SD}) \\
\end{array}$ & $\begin{array}{l}\mathbf{H C}_{\mathbf{T}}(\mathbf{n}=\mathbf{1 4}) \\
(\text { mean } \pm \text { SD }) \\
\end{array}$ & $\begin{array}{l}\mathbf{M S} \mathbf{u}(\mathbf{n}=\mathbf{1 4}) \\
(\text { mean } \pm \text { SD) } \\
\end{array}$ & $\begin{array}{l}\mathbf{M S}_{\mathbf{T}}(\mathbf{n}=\mathbf{1 5}) \\
(\text { mean } \pm \text { SD }) \\
\end{array}$ & Differences \\
\hline Age & $\begin{array}{l}34.13 \pm 6.07 \\
(25-45)\end{array}$ & $\begin{array}{c}31.21 \pm 8.72 \\
(24-50)\end{array}$ & $\begin{array}{l}36.14 \pm 5.97 \\
\quad(22-46)\end{array}$ & $\begin{array}{c}35.80 \pm 7.3 \\
(22-46)\end{array}$ & $\begin{array}{l}T: \mathrm{F}_{1,54}=0.76, \mathrm{p}=0.39 \\
G: \mathrm{F}_{1,54}=3.12, \mathrm{p}=0.08 \\
T x G: \mathrm{F}_{1,54}=0.48, \mathrm{p}=0.49\end{array}$ \\
\hline $\begin{array}{c}\text { Gender } \\
\text { (men/women) }\end{array}$ & $9 / 6$ & $6 / 8$ & $3 / 11$ & $7 / 8$ & $\chi^{2}(3)=4.51, p=0.21$ \\
\hline $\begin{array}{c}\text { Educational } \\
\text { level } \\
\text { (1-6 levels) }\end{array}$ & $3.73 \pm 1.28$ & $4.71 \pm 0.83$ & $3.71 \pm 1.49$ & $3.73 \pm 1.71$ & $\begin{array}{l}T: \mathrm{F}_{1,54}=1.93, \mathrm{p}=0.17 \\
G: \mathrm{F}_{1,54}=1.93, \mathrm{p}=0.17 \\
T x G: \mathrm{F}_{1,54}=1.78, \mathrm{p}=0.19\end{array}$ \\
\hline EDSS & - & - & $1.80 \pm 1.70$ & $1.67 \pm 1.51$ & $\mathrm{t}_{27}=0.19, \mathrm{p}=0.85$ \\
\hline $\begin{array}{c}\text { Disease } \\
\text { duration (years) }\end{array}$ & - & - & $7.54 \pm 5.12$ & $8.33 \pm 5.96$ & $\mathrm{t}_{27}=0.38, \mathrm{p}=0.85$ \\
\hline $\begin{array}{c}\text { Total lesion } \\
\text { volume }(\mathrm{mL})\end{array}$ & - & - & $4.39 \pm 4.88$ & $2.36 \pm 3.56$ & $\mathrm{t}_{27}=1.28, \mathrm{p}=0.21$ \\
\hline BPF & $0.86 \pm 0.01^{\mathrm{C}, \mathrm{D}}$ & $0.85 \pm 0.02^{\mathrm{C}, \mathrm{D}}$ & $0.84 \pm 0.02$ & $0.84 \pm 0.01$ & $\begin{array}{l}T: \mathrm{F}_{1,54}=0.19, \mathrm{p}=0.67 \\
\boldsymbol{G}: \mathbf{F}_{1,54}=\mathbf{1 0 . 3 0}, \mathbf{p}=\mathbf{0 . 0 0 2} \\
T x G: \mathrm{F}_{1,54}=0.37, \mathrm{p}=0.55\end{array}$ \\
\hline
\end{tabular}




\begin{tabular}{|c|c|c|c|c|c|}
\hline BRN-B & & & & & \\
\hline SDMT & $\begin{array}{r}59.69 \pm 9.09 \\
60.80\end{array}$ & $\begin{array}{l}66.17 \pm 6.37 \\
10.13\end{array}$ & $54.93 \pm 10.56$ & & $\begin{array}{l}T: \mathrm{F}_{1,54}=3.81, \mathrm{p}=0.06 \\
G: \mathrm{F}_{1,54}=3.12, \mathrm{p}=0.08 \\
T x G: \mathrm{F}_{1,54}=0.48, \mathrm{p}=0.49\end{array}$ \\
\hline PASAT (\%) & $\begin{array}{r}76.22 \pm 8.87 \\
83.44\end{array}$ & $\begin{array}{l}78.33 \pm 17.45 \\
13.01\end{array}$ & $74.86 \pm 18.18$ & & $\begin{array}{l}T: \mathrm{F}_{1,54}=1.40, \mathrm{p}=0.24 \\
G: \mathrm{F}_{1,54}=0.17, \mathrm{p}=0.68 \\
T x G: \mathrm{F}_{1,54}=0.51, \mathrm{p}=0.48\end{array}$ \\
\hline $\begin{array}{l}\text { SRT Long- } \\
\text { Term Storage }\end{array}$ & $\begin{array}{r}58.46 \pm 8.08 \\
52.53\end{array}$ & $\begin{array}{l}52.67 \pm 12.94 \\
10.29\end{array}$ & $52.07 \pm 13.53$ & & $\begin{array}{l}T: \mathrm{F}_{1,54}=0.60, \mathrm{p}=0.44 \\
G: \mathrm{F}_{1,54}=0.89, \mathrm{p}=0.35 \\
T x G: \mathrm{F}_{1,54}=0.82, \mathrm{p}=0.37\end{array}$ \\
\hline $\begin{array}{l}\text { SRT Consistent } \\
\text { Long-Term } \\
\text { Retrieval } \\
\end{array}$ & $\begin{array}{r}51.31 \pm 11.76 \\
42.07 \\
\end{array}$ & $\begin{array}{l}47.83 \pm 5.71 \\
2.32\end{array}$ & $43.21 \pm 14.75$ & & $\begin{array}{l}T: \mathrm{F}_{1,54}=0.31, \mathrm{p}=0.58 \\
G: \mathrm{F}_{1,54}=2.82, \mathrm{p}=0.10 \\
T x G: \mathrm{F}_{1,54}=0.08, \mathrm{p}=0.78\end{array}$ \\
\hline $\begin{array}{l}\text { SRT Delayed } \\
\text { Recall }\end{array}$ & $10.23 \pm 1.92$ & $10.50 \pm 1.98$ & $9.50 \pm 2.85$ & $10.27 \pm 1.98$ & $\begin{array}{l}T: \mathrm{F}_{1,54}=0.55, \mathrm{p}=0.46 \\
G: \mathrm{F}_{1,54}=0.48, \mathrm{p}=0.49 \\
T x G: \mathrm{F}_{1,54}=0.13, \mathrm{p}=0.72\end{array}$ \\
\hline $\begin{array}{l}\text { SPART Long- } \\
\text { Term Storage }\end{array}$ & $20.62 \pm 6.64$ & $23.33 \pm 3.26$ & $20.57 \pm 5.06$ & $20.33 \pm 5.18$ & $\begin{array}{l}T: \mathrm{F}_{1,54}=0.55, \mathrm{p}=0.46 \\
G: \mathrm{F}_{1,54}=0.83, \mathrm{p}=0.37 \\
T x G: \mathrm{F}_{1,54}=0.78, \mathrm{p}=0.38\end{array}$ \\
\hline $\begin{array}{l}\text { SPART } \\
\text { Delayed-Recall }\end{array}$ & $7.08 \pm 2.72$ & $8.67 \pm 1.75$ & $7.07 \pm 2.12$ & $7.27 \pm 1.83$ & $\begin{array}{l}T: \mathrm{F}_{1,54}=1.75, \mathrm{p}=0.19 \\
G: \mathrm{F}_{1,54}=1.08, \mathrm{p}=0.30 \\
T x G: \mathrm{F}_{1,54}=1.07, \mathrm{p}=0.31\end{array}$ \\
\hline WLGT & $22.54 \pm 3.57$ & $25.17 \pm 3.66$ & $21.14 \pm 6.29$ & $21.40 \pm 5.58$ & $\begin{array}{l}T: \mathrm{F}_{1,54}=0.82, \mathrm{p}=0.37 \\
G: \mathrm{F}_{1,54}=2.63, \mathrm{p}=0.11 \\
T x G: \mathrm{F}_{1,54}=0.55, \mathrm{p}=0.46\end{array}$ \\
\hline BDI & $7.85 \pm 5.65^{\mathrm{C}, \mathrm{D}}$ & $4.50 \pm 5.24^{\mathrm{C}, \mathrm{D}}$ & $14.21 \pm 7.98$ & $11.47 \pm 8.33$ & $\begin{array}{l}T: \mathrm{F}_{1,54}=1.84, \mathrm{p}=0.18 \\
\boldsymbol{G}: \mathbf{F}_{\mathbf{1}, \mathbf{5 4}}=\mathbf{8 . 8 3}, \mathbf{p}=\mathbf{0 . 0 0 5} \\
T x G: \mathrm{F}_{1,54}=0.02, \mathrm{p}=0.89\end{array}$ \\
\hline FSS & - & $\begin{array}{c}- \\
40.80 \pm 17.98\end{array}$ & $47.36 \pm 16.01$ & & $\mathrm{t}_{27}=1.03, \mathrm{p}=0.310$ \\
\hline $\begin{array}{l}\text { Matrix Subtest } \\
\text { (WAIS III) }\end{array}$ & $105.71 \pm 14.79$ & $106.43 \pm 16.34$ & $111.15 \pm 7.95$ & $106.33 \pm 12.17$ & $\begin{array}{l}T: \mathrm{F}_{1,54}=0.34, \mathrm{p}=0.56 \\
G: \mathrm{F}_{1,54}=0.57, \mathrm{p}=0.45 \\
T x G: \mathrm{F}_{1,54}=0.61, \mathrm{p}=0.44\end{array}$ \\
\hline
\end{tabular}

$T=$ Training factor; $G=$ Group factor; $T x G=$ Interaction; $H C u: H C$ untrained group; $H C t: H C$ trained group; MSu: MS untrained group; MSt: MS trained group; Educational level: $1=$ Primary education, $2=$ Lower secondary education, 3=Upper secondary education, 4=Post-secondary education non-tertiary, 5=First stage of tertiary education, 6=Second stage of tertiary education; EDSS: Expanded Disability Status Scale; BPF: Brain Parenchymal Fraction; BRN-B: The Brief Repeatable Battery of Neuropsychological Test; SDMT: Symbol Digit Modalities Test; PASAT: Paced Auditory Serial Addition Test; SRT: Selective Reminding Test; SPART: Spatial Recall Test; WLGT: Word List Generation Test. BDI: Beck Depression Inventory; FSS: Fatigue Severity Scale. A denotes statistically significant different from the $\mathrm{HCu}$ group; $B$ denotes statistically significant different from the HCt group; C denotes statistically significant different from the MSu group; D denotes statistically significant different from the MSt group. 


\section{$3.2 \mathrm{~N}$-back performance}

Table 2 shows the descriptive statistics (means and standard deviations) of each group for the number of CRs and RTs during the execution of the 2- and 3-back tasks inside the scanner at S1 and S2.

Regarding the number of CRs during the 2-back performance, a mixed two-way ANOVA (group $\mathrm{x}$ training $\mathrm{x}$ session) only revealed a main effect of session $\left[\mathrm{S} 2>\mathrm{S} 1 ; \mathrm{F}_{\mathrm{session}(1,54)}=16.119, p=1.85^{-3}\right.$, $\eta^{2}=0.082$ ), although the effects of training and the training $\mathrm{x}$ session interaction also approached statistical significance $\left[\mathrm{F}_{\text {training(1,54) }}=2.821, \mathrm{p}=0.099 ; \mathrm{F}_{\text {training } \mathrm{x} \text { session }(1,54)}=2.933, p=0.092\right)$. These effects were more evident for RT, where not only the main effects of training and session reached statistical significance, but also their interaction $\left[F_{\text {training } x \text { session }(1,54)}=21.30, p=2.46^{-4}, \eta^{2}=0.059\right]$. Subsequent post-hoc comparisons revealed that trained participants (MSt and $\mathrm{HCt}$ ) exhibited shorter RTs in S2 $\left(\mathrm{T}<\mathrm{U}, p=3^{-5}, d=-1.36\right)$ than in $\mathrm{S} 1(\mathrm{~T} \approx \mathrm{U}, p>0.05)$.

Cognitive training also improved 3-back performance. Regarding the number of CRs, a mixed twoway ANOVA (group $x$ training $x$ session) yielded a statistically significant effect of $\operatorname{session}(\mathrm{p}<0.001)$

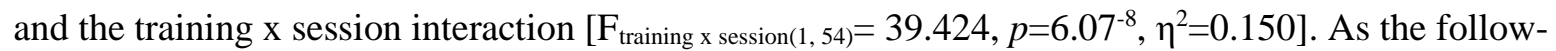
up comparisons show, this interaction effect was due to the higher number of CRs observed in trained participants in $\mathrm{S} 2\left(\mathrm{~T}>\mathrm{U}, p=1.03^{-5}, d=1.27\right)$, despite the lack of a statistically significant difference between trained and untrained participants in $\mathrm{S} 1(\mathrm{~T} \approx \mathrm{U}, p>0.05)$. The same pattern of results was observed for RT. In this case, the training and session factors and the training $\mathrm{x}$ session interaction reached statistical significance $\left[F_{\text {training } x \operatorname{session}(1,54)}=23.321, p=1.17^{-5}, \eta^{2}=0.06\right]$. Again, this interaction effect was due to the shorter RTs exhibited by trained participants in $\mathrm{S} 2$ ( $\left.\mathrm{T}<\mathrm{U}, p=3.72^{-8}, d=-1.67\right)$, but not in $\mathrm{S} 1(\mathrm{~T} \approx \mathrm{U}, p>0.05)$. 
Table 2. Correct responses (CRs) and reaction times (RTs) during the execution of 2 and 3 back tasks. Differences between groups were analysed using Analysis of Variance (ANOVA) and are described in the text (behavioral fMRI results).

\begin{tabular}{|c|c|c|c|c|}
\hline \multicolumn{5}{|c|}{ 2-back } \\
\hline & \multicolumn{2}{|c|}{ CRs } & \multicolumn{2}{|c|}{ RTs } \\
\hline & S1 & S2 & S1 & S2 \\
\hline $\mathrm{HCu}$ & $13.53 \pm 3.37$ & $14.66 \pm 2.84$ & $619.37 \pm 103.33$ & $570.30 \pm 104.63$ \\
\hline HCt & $14.42 \pm 2.90$ & $16.71 \pm 2.05$ & $625.72+155.27$ & $467.61 \pm 104.41$ \\
\hline MSu & $14.64 \pm 3.73$ & $15.28 \pm 2.25$ & $708.46 \pm 129.91$ & $707.37 \pm 141.62$ \\
\hline MSt & $14.40 \pm 1.76$ & $16.53 \pm 1.99$ & $606.91 \pm 115.75$ & $482.77 \pm 79.57$ \\
\hline \multicolumn{5}{|c|}{3 -back } \\
\hline & \multicolumn{2}{|c|}{ CRs } & \multicolumn{2}{|c|}{$\overline{R T s}$} \\
\hline & S1 & S2 & S1 & S2 \\
\hline $\mathrm{HCu}$ & $13.13 \pm 3.99$ & $12.60 \pm 4.17$ & $674.93 \pm 144.92$ & $609.09 \pm 109.46$ \\
\hline HCt & $11.00 \pm 3.55$ & $16.50 \pm 1.78$ & $640.30 \pm 162.14$ & $451.34 \pm 90.59$ \\
\hline MSu & $13.14 \pm 3.32$ & $13.28 \pm 2.78$ & $737.95 \pm 142.51$ & $723.42 \pm 138.63$ \\
\hline MSt & $11.20 \pm 4.64$ & $16.33 \pm 1.44$ & $629.90 \pm 154.05$ & $471.58+118.75$ \\
\hline
\end{tabular}

\subsection{Between-group differences in FC}

Table 3 and Figure 1 report all the statistically significant effects obtained in the mixed two-way ANOVAs (group $\mathrm{x}$ training $\mathrm{x}$ session) comparing the $\mathrm{FC}$ scores of the four participant groups during the 2- and 3-back tasks. Similarly, to what was observed for the n-back performance indexes (CRs and RT), between-group differences in FC scores arose from training $\mathrm{x}$ session interactions, but they were largely independent of the group factor (HC vs MS).

Thus, during the 2-back performance, the only statistically significant effect involving the group factor was a group $\mathrm{x}$ training $\mathrm{x}$ session interaction in the $\mathrm{FC}$ between the left insula and the left precentral gyrus. Follow-up analyses revealed that this effect was solely due to differences between the two groups of MS patients. Moreover, trained participants (HCt and MSt) exhibited stronger FC than untrained participants $(\mathrm{HCu}$ and $\mathrm{MSu})$ in several fronto-parietal areas in $\mathrm{S} 2$, but not in $\mathrm{S} 1$ (training x session interaction, see Table 3). Specifically, trained groups showed increased FC between the left precentral gyrus and left insula, left precuneus, and left precentral gyrus, between the left insula and right inferior frontal gyrus, and between the left superior parietal lobe and left precuneus. The size of these univariate differences added up to a multivariate $D=1.50$ [95\% CI: 0.7, 1.94], a small degree of overlap of the $\Delta$-FC score distributions of trained/ untrained participants $(45.2 \%)$, and a large PS for trained participants (0.86). Accordingly, $\Delta$-FC scores allowed us to 
correctly identify trained/ untrained participants in $81.03 \%$ ([95\%CI: $0.69,0.90], p=1.016^{-6}$ ) of the cases.

Similar between-group differences were observed in FC during 3-back performance. Thus, in trained participants (HC and MS), stronger FC was observed between the left precuneus and left superior parietal lobe and the reverse contrast, as well as between the left insula and right inferior frontal gyrus (see Table 3 for further details). These univariate differences added up, yielding a $\mathrm{D}=1.15$ [95\%CI: $0.44,1.58]$, which translated into a degree of multivariate overlap of the $\Delta$-FC score distributions of trained/ untrained participants equal to $56.6 \%$, and to a moderate-to-large PS for trained participants (0.79). These $\Delta$-FC scores led to $79.31 \%$ ([95\%CI: $0.67,0.89], \mathrm{p}=4.11-6$ ) of cases being correctly classified.

No other statistically significant differences between groups were observed. 


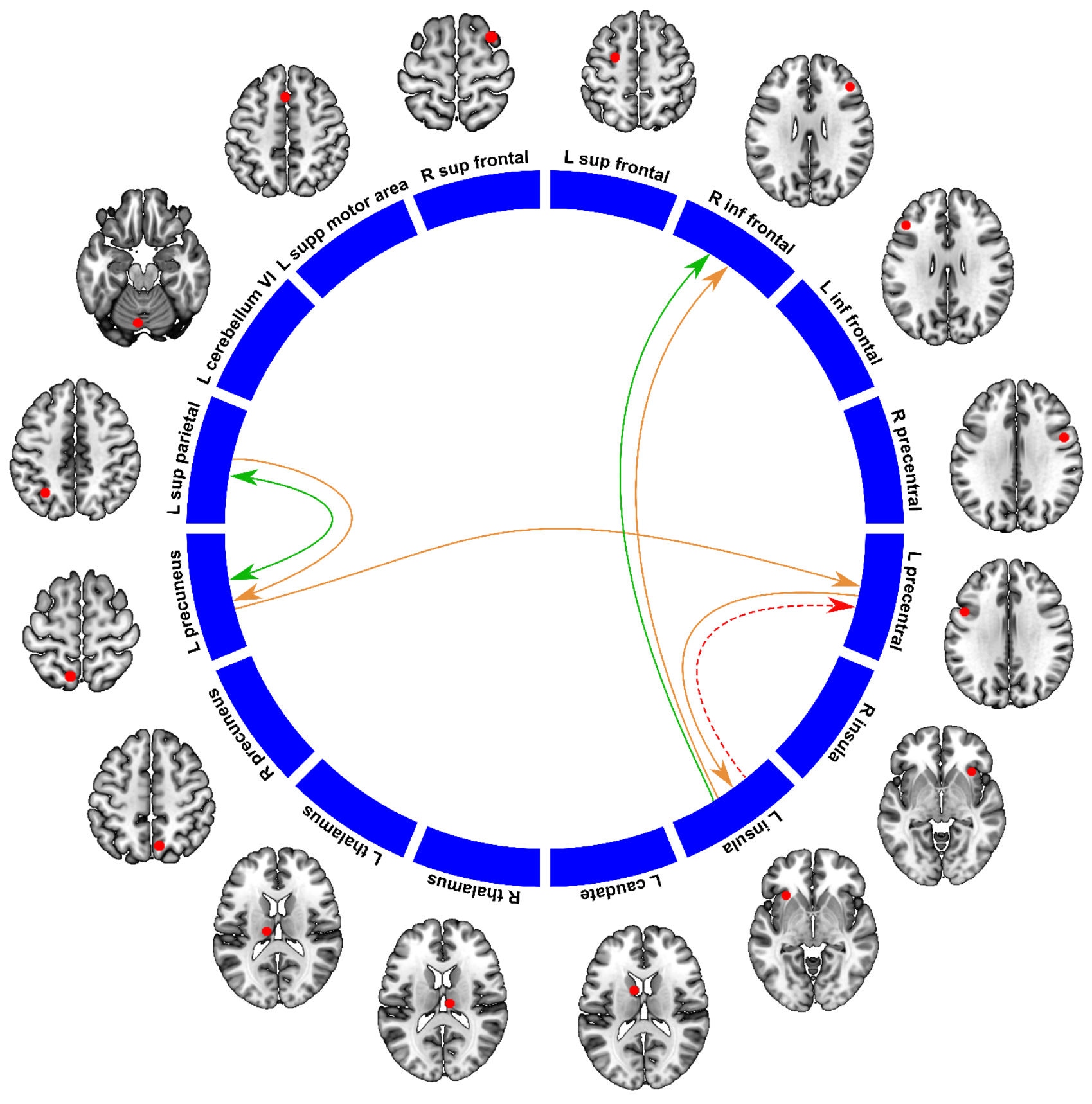

Figure 1. Connectivity between ROIs during 2- and 3-back tasks. Solid line arrows represent stronger FC in trained participants ( $\mathrm{HCt}$ and $\mathrm{MSt}$ ) compared to untrained participants (Training*Time effects) during the execution of 2-back (orange) and 3-back (green) tasks. The red dashed line arrow represents differences in FC between MSu and MSt (Group*Training*Time effect) during the 2-back task. Results were $\mathrm{p}<0.05$, FWE-corrected. 
Table 3. Between groups differences in functional connectivity (FC) during 2 and 3 -back tasks. The table displays the statistically significant ( $\mathrm{p}<0.05$, FWE-corrected) ANOVA effects for FC scores.

\begin{tabular}{|c|c|c|c|}
\hline \multicolumn{4}{|c|}{2 -back } \\
\hline Anatomical regions & Training x Session effects & S1 & $\mathbf{S 2}$ \\
\hline L precentral gyrus-L insula & $\mathrm{F}_{1,54}=10.91, p=0.032, \eta^{2}=0.07$ & $\begin{array}{l}\mathrm{T} \approx \mathrm{U} \\
p>0.05\end{array}$ & $\begin{array}{c}\mathrm{T}>\mathrm{U} \\
p=0.016, d=0.65\end{array}$ \\
\hline $\begin{array}{l}\text { L insula- } R \text { inferior frontal } \\
\text { gyrus (triangularis) }\end{array}$ & $\mathrm{F}_{1,54}=9.71, p=0.048, \eta^{2}=0.07$ & $\begin{array}{l}\mathrm{T} \approx \mathrm{U} \\
p>0.05\end{array}$ & $\begin{array}{c}\mathrm{T}>\mathrm{U}, \\
p=0.035, d=0.57\end{array}$ \\
\hline $\begin{array}{l}\text { L precuneus-L precentral } \\
\text { gyrus }\end{array}$ & $\mathrm{F}_{1,54}=13.88, p=0.007, \eta^{2}=0.09$ & $\begin{array}{c}\mathrm{T}<\mathrm{U} \\
p=0.014, d=-0.66\end{array}$ & $\begin{array}{c}\mathrm{T}>\mathrm{U} \\
p=0.047, d=0.53\end{array}$ \\
\hline \multirow[t]{2}{*}{$\begin{array}{l}\text { L superior parietal lobe- } \\
\text { L precuneus }\end{array}$} & $\mathrm{F}_{1,54}=10.47, p=0.035, \eta^{2}=0.11$ & $\begin{array}{l}\mathrm{T} \approx \mathrm{U} \\
p>0.05\end{array}$ & $\begin{array}{c}\mathrm{T}>\mathrm{U} \\
p=0.003, d=0.83\end{array}$ \\
\hline & Group x Training x Session effects & S1 & S2 \\
\hline L insula-L precentral gyrus & $\mathrm{F}_{1,54}=10.75, p=0.032, \eta^{2}=0.08$ & $\begin{array}{c}\mathrm{MSu}>\mathrm{MSt} \\
p=0.013, d=-0.99\end{array}$ & $\begin{array}{c}\mathrm{MSt}>\mathrm{MSu} \\
p=0.005, d=1.13\end{array}$ \\
\hline \multicolumn{4}{|c|}{3 -back } \\
\hline Anatomical regions & Training x Session effects & S1 & $\mathbf{S 2}$ \\
\hline $\begin{array}{l}\text { L precuneus-L superior } \\
\text { parietal lobe }\end{array}$ & $\mathrm{F}_{1,54}=11.31, p=0.016, \eta^{2}=0.10$ & $\begin{array}{l}\mathrm{T} \approx \mathrm{U} \\
p>0.05\end{array}$ & $\begin{array}{c}\mathrm{T}>\mathrm{U} \\
p=0.002, d=0.83\end{array}$ \\
\hline $\begin{array}{l}\text { L superior parietal lobe- } \\
\text { L precuneus }\end{array}$ & $\mathrm{F}_{1,54}=10.44, p=0.032, \eta^{2}=0.09$ & $\begin{array}{l}\mathrm{T} \approx \mathrm{U} \\
p>0.05\end{array}$ & $\begin{array}{c}\mathrm{T}>\mathrm{U} \\
p=0.01, d=0.69\end{array}$ \\
\hline $\begin{array}{l}\text { L insula- } R \text { inferior } \\
\text { frontal gyrus (triangularis) }\end{array}$ & $\mathrm{F}_{1,54}=9.82, p=0.048, \eta^{2}=0.08$ & $\begin{array}{c}\mathrm{T} \approx \mathrm{U} \\
p>0.05\end{array}$ & $\begin{array}{c}\mathrm{T}>\mathrm{U} \\
p=0.006, d=0.75\end{array}$ \\
\hline
\end{tabular}

$L=$ left; R=Right; T=trained groups (all trained participants that included MSt and HCt); U=Untrained participants (all untrained participants that included $\mathrm{MSu}$ and $\mathrm{HCu}$ ); $\mathrm{SI}=$ session one (pre-training); $S 2=$ session 2 (post-training) 


\subsection{Relationships between FC and n-back performance}

As Figure 2 shows, the training-induced changes (S2 -S1 difference) in FC and in the n-back performance indexes (CRs and RT) were related.

Regarding 2-back performance, the S2-S1 change in the number of CRs ( $\Delta$-CRs) was directly and significantly correlated with the $\Delta$-FC between the left precuneus and the left precentral gyrus. All the other statistically significant FC changes were unrelated to the $\Delta$-CR. Thus, the combination of all these $\Delta$-FC scores in a single predictive model explained a relatively low amount of the variance in $\Delta$-CRs (deviance explained $=10.4 \%$; adjusted $\mathrm{R}^{2}=0.090$; "small" effect size). On the other hand, the S2-S1 change in the RT ( $\Delta$-RT) was inverse and significantly correlated with the $\Delta$-FC between the left precentral and the left insula, between the left insula and the right inferior frontal gyrus, and between the left superior parietal lobe and the left precuneus. The $\Delta$-FC between the left precuneus and the left precentral was only marginally correlated $(p=0.06)$ with the $\Delta-\mathrm{RT}$. Taken together, these FC changes accounted for a large amount of $\Delta$-RT variance (deviance explained $=23.6 \%$, adjusted $\mathrm{R}^{2}=0.18$; "medium" effect size). Finally, although it is not shown in Figure 1, the $\Delta$-FC between the left insula and the left precentral gyrus observed in MS patients was inversely correlated with their $\Delta$-RT $(r=-0.44, p=0.016)$.

Regarding 3-back performance, the S2 vs. S1 changes in the number of CRs were directly and significantly correlated with the $\Delta$-FC between the left insula and the right inferior frontal gyrus, between the left superior parietal lobe and the left precuneus, and between the left precuneus and the left superior parietal lobe. In this case, the deviance in $\Delta$-CRs explained by the $\Delta$-FC scores was $24.4 \%$ (adjusted $\mathrm{R}^{2}=0.22$; "medium" effect size). In addition, these $\Delta$-FC scores also showed inverse and statistically significant correlations with the S2-S1 RT changes. Combining these $\Delta$-FC scores into a single predictive model allowed us to account for $34.6 \%$ of the deviance in $\Delta$-CRs (adjusted $\mathrm{R}^{2}=0.29$; “medium” effect size). 


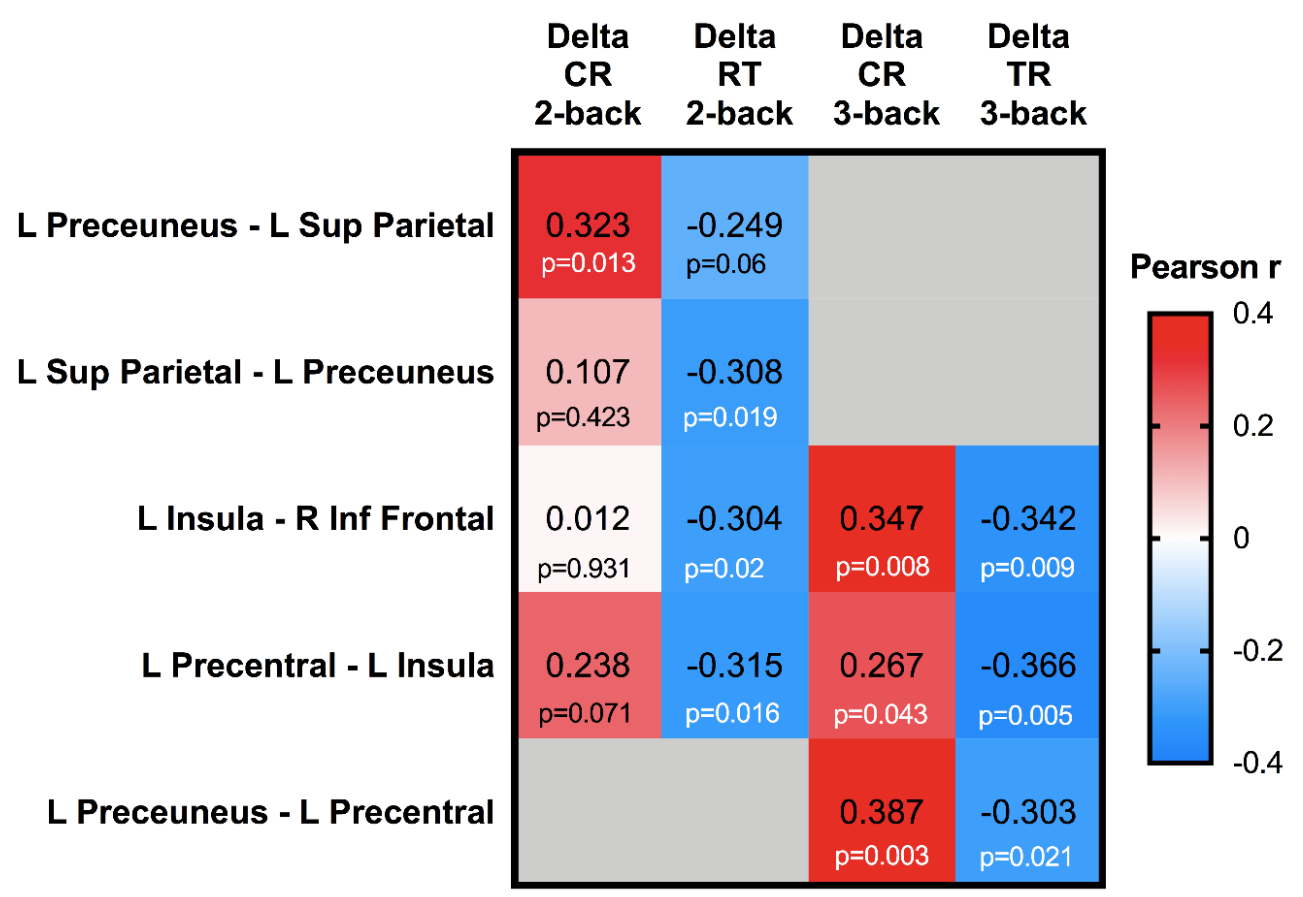

Figure 2. Relationship between training-induced changes in FC and in n-back performance. The figure displays the values of Pearson's $r$ correlation index quantifying the relationship between $\mathrm{S} 2-\mathrm{S} 1$, the gains in performance $(\Delta-\mathrm{CRs}$ and $\Delta$-RT), and the S2-S1 changes in FC summarized in Table 3. The $r$ values and their associated $p$-values are reported inside the cells. Cells colored in red and blue tones illustrate direct and inverse correlations, respectively. Gray colored cells denote FC paths that did not reach statistical significance on either the 2-back or 3-back task.

\section{Discussion}

This study reveals the benefits of specific and intensive computerized WMF training in a group of cognitively preserved MS patients and HC. First, we observed that, after four days (60 min/ day) of intensive training specifically focused on WM, both MS patients and HC improved their WMF performance, exhibiting greater CRs rates and lower RTs. Second, we observed that the same training program led to an increase in neural FC in the fronto-parietal network belonging to WM. In addition, we also observed that the two kinds of training-induced changes (better performance and stronger FC) were associated with each other.

Compared to the non-trained participants, trained participants (HC and MS) showed enhanced n-back performance in S2 compared to S1. These effects were observed as an increase in the CRs rate (which provides a purer measure of WM) and as a RT decrease (which might be interpreted as reflecting an enhancement of IPS). These results confirm and extend those from other previous studies using this 
specific WMF training program (Aguirre, Cruz-Gómez, et al., 2019; Covey et al., 2018; T. W. Thompson et al., 2016).

As expected, cognitive training resulted in increased FC during the execution of the 2- and 3-back tasks. With the only exception of a selective enhancement of the FC between the left insula and left precentral gyrus observed in trained MS patients (but not in HC) during 2-back (but not 3-back) performance, the effects of training on FC were quite similar in all the trained participants. Thus, trained HC and trained MS patients exhibited increased FC between superior parietal areas (including the precuneus) and between parietal and frontal areas (including the precentral gyrus, the inferior frontal gyrus, and insula). The precuneus is involved in shifting attention processes, especially when the targets objectives are presented in different locations in the space and required motor responses (Cavanna \& Trimble, 2006). The left precentral gyrus (that comprises part of the primary motor cortex) is related to hand movement and, as we also observed in the present study, its activity had been previously associated to lower RTs in WMF tasks (Emch et al., 2019). On the other hand, the insula has an important function in multimodal sensory processes and it has a similar role to that of the precentral gyrus in hand, but also, in eye movement and language processing (Oh et al., 2014; Wang et al., 2019). Moreover, the left insula is part of the articulatory loop and, as such, it is considered as having an essential functional role in WMF tasks (Emch et al., 2019). Finally, the inferior frontal gyrus plays an important role in inhibitory processes, which are necessary to suppress context-inappropriate responses and adequately perform WMF tasks (e.g. to avoid reporting the stimulus presented in the 2- and 3-back tasks (Hampshire et al., 2010; Levy \& Wagner, 2011)). These FC changes conformed a clearly defined pattern that made it possible to correctly distinguish trained and non-trained participants in $\approx 80 \%$ of the cases. Interestingly, these training-induced S2-S1 FC increases were directly correlated with the S2-S1 gains in CRs rates and inversely related to the S2S1 RT changes. In fact, these $\square$-FC scores additively explained up to $24.4 \%$ and $29 \%$ of the variance in the $\Delta$-CRs and $\Delta$-RT, respectively.

Taken together, the results of the present study confirm and extend previous findings showing that cognitive training leads to enhanced FC and improved task performance in MS patients (Bonavita et al., 2015; De Giglio, Tona, et al., 2016; Leavitt, Wylie, Girgis, et al., 2014; Parisi, Rocca, Valsasina, et al., 2014). Moreover, and in agreement with previous studies conducted in healthy volunteers (Constantinidis \& Klingberg, 2016), we observed that a WMF training increased FC between frontoparietal areas and this FC increase was significantly correlated to the training-induce performance improvements. 
Although the present results are encouraging and confirm the efficacy of training to stimulate brain plasticity mechanisms that can enhance cognitive performance in MS patients, the present study also has some limitations that should be considered. First, as in most studies that assess the effects of cognitive training programs in MS patients, the recruited sample was small. Therefore, the statistical power achieved might be suboptimal, and it is likely that this study failed to identify all the relevant between-group differences in FC.

Second, we recruited homogeneous groups of participants. Specifically, all of the MS patients were diagnosed with the RR phenotype, presented a few years of disease evolution, and were cognitively preserved (the neuropsychological performance of these patients was indistinguishable from that of the HC, and no statistically significant differences between MS and HC were found on any cognitive test). Although the use of homogenous groups reduces spurious variability, partially counteracts the negative effects of the reduced sample size, and facilitates the identification of between-group differences, it also reduces the generalizability of the results (Carter et al., 2015). Thus, we cannot conclude that this training protocol would improve WMF and IPS to the same degree in MS patients with different clinical characteristics, particularly those with severe cognitive impairment. Similarly, the present study does not provide information about a possible moderating role of potentially relevant variables (e.g. age, gender, cognitive reserve, atrophy, etc.) in the beneficial effects of this cognitive training program.

Third, in the present study, the possible long-term effects of our WMF training program were not examined. In this regard, future studies should determine to what extent the observed FC changes and task performance enhancement persist over time.

Finally, WMF capabilities largely underlie and subserve other cognitive abilities, and as previously suggested (Covey et al., 2018; Mitolo et al., 2015), the training-induced WMF improvements could lead to improvements in other cognitive domains. However, although we observed a reduction in RT that might be interpreted as being the result of IPS improvement, we did not specifically test these possible generalization or transfer effects to other cognitive processes. Future studies should specifically assess the extent to which WMF training improves other cognitive functions and other important cognitive rehabilitation goals in MS patients, such as perceived quality of life or emotional/mood status. Furthermore, in this study we used and intensive training, that only included four consecutive days. In this sense, future studies should explore the efficacy of training programs with different intensities/duration which could be beneficial for groups of patients with a higher degree of cognitive impairment (moderate/ severe). 
In conclusion, this study reinforces the notion that short periods of cognitive training could be useful to improve cognitive functions and brain resources in patients with brain damage. More specifically, implementing early interventions in MS patients, could be an useful strategy to prevent or attenuate the cognitive decline in diagnosed MS patients (Covey et al., 2018; Lampit et al., 2019).

\section{Acknowledgments}

We would like to extend our gratitude to AEMC and ASCIRES ERESA Castellón for their collaboration in the present study.

\section{Financial support}

This research was supported by a grant [PSI2015-67285-R] provided by Ministerio de Educación, Cultura y Deporte to C.F and by a grant [UJI B2017-05] awarded to C.S-S. N.A was supported by an FPU grant from the Ministerio de Educación [FPU16/01525]. E.B was funded by a postdoctoral research grant from the Generalitat Valenciana and the European Social Fund ["Investing in your future"; 2018 APOSTD].

\section{Conflicts of Interest}

The authors declare that there are no conflicts of interest

\section{Authors' contributions}

C.F and C.A contributed to the planning and preparation of the current study and also conceived the experimental design. A.M-P, E.B and C.A prepared the paradigm. The patients were selected by N.A and J.C-G. A.M-P, E.B, N.A, J.C-G R.B-T and SF-E were responsible for collecting the fMRI data. C.S-S and C.F performed the statistical analysis and contributed to the interpretation of the results. C.F, N.A and C.S-S generated the manuscript. C.F, C.S-S and N.A were intensely involved in the final version of the manuscript. All authors read and approved the final manuscript. 
CHAPTER 3

GENERAL DISCUSSION 


\section{GENERAL DISCUSSION}

WM lesions are the most characteristic pathology in MS patients; nevertheless, the relationship between these lesions and brain functionality or cognitive deficits is unclear. In fact, the mismatch between clinical outcomes and the prevalence of WM lesions has been consistently reported (Fulton et al., 1999; Heesen et al., 2010; Hulst et al., 2013; Mollison et al., 2017; Uher et al., 2014), and it is called the clinical-radiological paradox (Barkhof, 1999, 2002). In this context, previous studies with RRMS patients yield inconsistent findings; both FC increases and decreases have been reported related to the WM lesion load (Castellazzi et al., 2018; Louapre et al., 2014; Soares et al., 2021; Tahedl et al., 2018). In order to explain the diversity in the results obtained, the hypothesis of the inverse U-curve has been suggested (Fleischer et al., 2019; Tewarie et al., 2018). This hypothesis proposes an initial structural disconnection related to increased FC, associated with compensatory mechanisms that reach their limits due to the built-up damage resulting in decreases in overall FC. Although this interpretation fits the heterogenous and widespread lesion burden well, the precise spatial relationships between localized structural damage and FC remain largely unknown. Understanding how specific brain tissue lesions are related to FC changes requires further research on this topic (Fleischer et al., 2019; Soares et al., 2021; Tahedl et al., 2018). To contribute to this goal, in the first study included in this doctoral thesis, we used a novel network-wise approach to detect and investigate the topological impact of the location of WM-lesions on functional connectivity networks in MS, based on graph theory metrics (Bassett \& Sporns, 2017; Diez \& Sepulcre, 2018; Rubinov \& Sporns, 2010; Sepulcre, Sabuncu, \& Johnson, 2012; Tahedl et al., 2018). This framework allowed us to characterize key spatial integration features of disease-related structural and functional alterations in MS.

One of the major findings of this study was that lesions located in the internal capsule were consistently associated with FC changes in MS patients. Specifically, we observed that an increase in lesion burden in the left posterior limb of the internal capsule was associated with an increase in global FC, measured by WD-FC, in several regions of different resting state networks, including the DMN, FP, SMN, VAN, and VN. The posterior limb contains thalamocortical and corticospinal fibers, which have been shown to be related to cognitive impairment (Ricigliano et al., 2021; Sepulcre et al., 2009; Zhou et al., 2016) and physical disability in MS (Bonzano et al., 2014; DeLuca et al., 2004; Ganter et al., 1999; Spain et al., 2009). In order to identify whether the increases in FC correspond to an increase in overall FC or a secondary effect of disconnection between regions, characteristic of 
MS disease, we performed a modularity analysis that segregates the functional connections in local and distant connectivity. Local connectivity identifies dense connections between proximal GM voxels, whereas distant connectivity corresponds to sparse connections between distant GM voxels (Diez \& Sepulcre, 2018). Regarding this classification, our results revealed that the increases in WDFC obtained in our study corresponded to increases in local connectivity. Likewise, this analysis revealed increases in FC of the DAN that had not been observed by WD-FC analysis. This additional result indicates that segregating FC into its local and distal components provides relevant information that may go unnoticed when using methods that only consider changes in global FC.

Increases in local FC indicate stronger connections between proximal regions, which is in line with previous studies that define MS as a multiple disconnection syndrome (P. Calabrese \& Penner, 2007) and the aforementioned hypothesis of the inverse U-curve (Fleischer et al., 2019; Tewarie et al., 2018). In this way, the demyelinating lesions characteristic of the disease, mainly affecting long pathways, lead to a disconnection between distal regions that, in early stages of the disease is reflected in an increase in local FC. In this context, the increase in local FC benefits active changes, understood as compensatory mechanisms (Basile et al., 2014; Fleischer et al., 2017; Muthuraman et al., 2016; Rocca, Absinta, et al., 2010) that, with the cumulative damage accompanying disease progression, reach their limit, resulting in a decrease in FC. However, the increase in local FC can also be interpreted as passive changes, a by-product of the lesions. Regarding these possible interpretations, we explore the relationship between changes in FC and cognitive performance. Our results showed that increased FC was associated with lower scores on neuropsychological tests, specifically on the PASAT, SRT, and SPART tests. This relationship can be interpreted based on the two perspectives mentioned above. On the one hand, we are working with a sample of cognitively preserved MS patients in early stages of the disease whose performance on the neuropsychological assessment is indistinguishable from that of HC. This supports the idea that the observed increases in connectivity can be interpreted as compensatory mechanisms. Nevertheless, we used a methodology that explores changes in FC related to a pathological disease process, i.e., WM lesions. Likewise, the increases in FC were associated with worse performance in MS patients but not in HC. This is in line with the maladaptive processes interpretation, indicating that this methodology allows us to explore subtle changes in FC that may take place in the early stages of the disease when cognitive impairment has not yet been possible to determine with clinical criteria. Further research is required to clarify the meaning of these FC changes associated with specific WM lesions.

In this thesis, we would like to emphasize the importance of the cognitive domains associated with increases in local FC. On the one hand, the PASAT measures IPS and WMF, whereas the SRT and 
SPART tests measure learning and long-term memory capacity for verbal and visuospatial domains, respectively. These cognitive functions are the most affected in MS patients, even in early stages of the disease (Bergendal et al., 2007; Chiaravalloti \& DeLuca, 2008; Janculjak et al., 2002; Langdon, 2011). Among these cognitive domains, WMF and IPS are of particular importance in early stages of the disease. In fact, the IPS is the cognitive domain most widely affected by MS, and also the first cognitive deficit to emerge in MS (Van Schependom et al., 2015). Furthermore, reduced IPS have been reported as the primary alteration (DeLuca et al., 2004; Forn et al., 2008; Van Schependom et al., 2015) underlying alterations in several cognitive domains, such as WMF (Demaree et al., 1999; Leavitt et al., 2011), response inhibition (Denney \& Lynch, 2009), planning (Owens et al., 2013), task switching (Leavitt, Wylie, Krch, et al., 2014), or attention (Roth et al., 2015). Additionally, impaired WMF have been widely documented in MS (Lengenfelder et al., 2003; Litvan et al., 1988; Parmenter et al., 2006; Ruchkin et al., 1994; Vacchi et al., 2017), and the impairment of this cognitive function is closely linked to IPS deficits (Lengenfelder et al., 2006). This shows the importance of working on these functions in specific cognitive training programs.

In this regard, the CR programs normally used in MS patients focus on multi-domain interventions, targeting the most affected cognitive functions in MS patients, such as attention, IPS, WMF, executive functions, short-term memory, and episodic memory (Mitolo et al., 2015; Prosperini \& Filippo, 2019). However, the results of these studies are inconclusive, probably due to methodological weaknesses such as heterogeneity of the selected participants or the diversity and lack of specificity of the CR used. Taking these limitations into account, we tested the effectiveness of a WMF training program in a homogeneous group of MS with no signs of cognitive deficits and in early stages of the disease. We designed a specific and intensive cognitive training in WMF using an adaptive version of the nback task. We opted for a specific cognitive training because it has been suggested that CR programs targeting specific cognitive domains could maximize their effectiveness, and improving functions that play central roles in the cognitive architecture, such as IPS and WMF, will maximize the applicability of the interventions' effects (Mitolo et al., 2015). Specifically, we used performance measures (CRs and RTs) to study the effectiveness of the WMF training. We also explored neural plasticity processes underlying performance improvement through changes in brain activation (Study II), as well as changes in brain FC (Study III). In this context, MS patients showed a significant improvement, similar to $\mathrm{HC}$, in their performance on the WMF trained task. That is, participants exhibited lower RTs and an increase in CRs after WMF training, with the improvement being more pronounced on RTs than on CRs. Importantly, performance improvement was associated with a decrease in activation but an increase in connectivity in frontoparietal regions. 
On the one hand, the decrease in brain activity in frontoparietal areas accompanying improved performance can be interpreted in the framework of the neural efficiency hypothesis (Buschkuehl et al., 2012; Forn et al., 2013; Miró-Padilla et al., 2018). This concept refers to achieving maximum performance levels while deploying a minimum amount of brain resources. In this context, the WMF training program used in the present thesis increased both task performance and neural efficiency, directly supported by statistically significant correlations between the reduction in the activation in frontoparietal areas and the reduction in RTs. Considering the duration of the WMF training (4 days), these results support the hypothesis that programs targeting specific cognitive domains maximize their effectiveness. In other words, we observed a clear effect of the WMF training on the performance and brain activity of the MS patients that was also maintained over time (1-month follow-up). Furthermore, as mentioned above, improvement in performance was more pronounced in RTs than in CRs, and the decrease in brain activity was more closely related to RT reductions. RTs are associated with IPS (Hughes et al., 2011); therefore, the cognitive training proposed in this thesis produces changes in the most affected cognitive domain in early stages of MS, which underlies alterations in several cognitive functions.

On the other hand, MS patients showed enhanced FC in frontoparietal areas during n-back task execution, similar to $\mathrm{HC}$, which correlated with improved performance. Specifically, increases in FC correlated positively with CRs, whereas they showed negative correlations with RTs. These correlations directly support the idea that the observed changes in FC reflect an adaptive neuroplasticity mechanism. Interestingly, the increases in FC were mainly observed between proximal regions, such as the connectivity between the superior parietal lobe and precuneus, or the connectivity between the inferior frontal gyrus and insula, but enhanced FC was also observed between distal regions, such as the precuneus and precentral gyrus. As mentioned above, increases in FC in early stages of the disease, suggested in the hypothesis of the inverse U-curve, have been associated with increases in local connectivity, i.e., increases in FC between proximal regions (Muthuraman et al., 2016). Thus, the impairment of a long pathway affects the FC between distant regions within a brain network, leading to a decrease in distal connectivity in the network that is usually observed in advanced stages of the disease. However, in early stages of the disease, an increase in local connectivity can be found to be a compensatory mechanism for brain damage. In this context, our findings showed that the specific cognitive training proposed stimulates a brain plasticity mechanism that can enhance cognitive performance, at the same time that it contributes to compensating for both local and distal alterations in the FC underlying brain damage. 
In short, FC changes associated with the CR programs reported in the studies so far are highly variable. This is due to the variety of analytical methods used, which should be carefully interpreted, and so the use of correlations between FC measures and cognitive performance is recommended. This variability is closely related to methodological limitations. In other words, the FC methods requires larger samples to ensure statistical power for the results, and these methods are highly sensitive to sample characteristics; therefore, mixed MS phenotypes would affect the results. All these aspects should be taken into consideration in the interpretation of FC analyses. In light of these limitations, we explored the effectiveness of a WMF training program in a homogeneous sample of cognitively unimpaired MS patients in early stages of the disease, assessing brain function and task performance before and immediately after the cognitive training. Additionally, we used different methodological approaches to study underlying neural plasticity processes associated with the WMF training, based on brain activity and functional connectivity. Likewise, we explored the effect of the WM-lesions on the FC, given their importance in the interpretation of the results. Another strength of the studies included in this thesis is the correlations between measures of brain activation and FC and participants' performance, which allows us to clearly interpret the changes observed at the level of activation and brain connectivity. Nevertheless, the present thesis has some limitations that should be considered in future studies. First, as in previous studies, the recruited sample was small. The statistical power achieved was suboptimal, and it is likely that we failed to identify all the relevant between-group differences in FC. Although it is a common issue, especially in studies with MS patients that include CR programs, this problem may be addressed by using statistical models adjusted to nonparametric samples. Second, we recruited MS patients with a homogeneous profile; specifically, all of the MS patients were diagnosed with the RR phenotype, presented few years of disease evolution, and were cognitively preserved. Although the use of homogenous groups reduces spurious variability, partially counteracts the negative effects of the reduced sample size, and facilitates the identification of between-group differences, it also reduces the generalizability of the results (Carter et al., 2015). In this regard, future studies should explore the effect of specific cognitive training programs in other MS phenotypes with different degrees of cognitive impairment. Lastly, as mentioned above, our cognitive training targeted WMF, which largely underlies and subserves other cognitive functions. Furthermore, this cognitive function is inseparable from IPS, which is a primary cognitive domain underlying a wide variety of cognitive functions. In this research, we have not tested possible generalization or transfer effects to other cognitive processes. However, in this case, because the MS patients recruited for the study did not show signs of cognitive deficits, we did not expect significant changes in cognitive performance in other cognitive domains. In any case, it would be interesting to study transfer effects in future research. 


\section{REFERENCES}

(US), I. of M. (2001). Multiple Sclerosis: Current Status and Strategies for the Future. (J. Joy \& R. J. Johnston (eds.)). National Academies Press. https://doi.org/10.17226/10031

Abel, S., Vavasour, I., Lee, L. E., Johnson, P., Ackermans, N., Chan, J., Dvorak, A., Schabas, A., Wiggermann, V., Tam, R., Kuan, A. J., Morrow, S. A., Wilken, J., Laule, C., Rauscher, A., Bhan, V., Sayao, A. L., Devonshire, V., Li, D. K., ... Kolind, S. H. (2020). Myelin Damage in Normal Appearing White Matter Contributes to Impaired Cognitive Processing Speed in Multiple Sclerosis. Journal of Neuroimaging, 30(2), 205-211. https://doi.org/10.1111/jon.12679

Absinta, M., Sati, P., Masuzzo, F., Nair, G., Sethi, V., Kolb, H., Ohayon, J., Wu, T., Cortese, I. C. M., \& Reich, D. S. (2019). Association of Chronic Active Multiple Sclerosis Lesions with Disability in Vivo. JAMA Neurology, 76(12), 1474-1483. https://doi.org/10.1001/jamaneurol.2019.2399

Agamanolis, D. P. (2019). Chapter 6 Demyelinative diseases. In An illustrated interactive course for medical students and residents. https://neuropathologyweb.org/chapter6/chapter6aMs.html

Aguirre, N., Costumero, V., Marin-Marin, L., Escudero, J., Belloch, V., Parcet, M. A., \& Ávila, C. (2019). Activity in Memory Brain Networks during Encoding Differentiates Mild Cognitive Impairment Converters from Non-Converters. Journal of Alzheimer's Disease, 71(3), 10491061. https://doi.org/10.3233/JAD-190421

Aguirre, N., Cruz-Gómez, Á. J., Esbrí, S. F., Miró-Padilla, A., Bueichekú, E., Broseta-Torres, R., Ávila, C., Sanchis-Segura, C., \& Forn, C. (2021). Enhanced frontoparietal connectivity in multiple sclerosis patients and healthy controls in response to an intensive computerized training focused on working memory. Multiple Sclerosis and Related Disorders, 52, 102976. https://doi.org/10.1016/j.msard.2021.102976

Aguirre, N., Cruz-Gómez, Á. J., Miró-Padilla, A., Bueichekú, E., Broseta Torres, R., Ávila, C., Sanchis-Segura, C., \& Forn, C. (2019). Repeated Working Memory Training Improves Task Performance and Neural Efficiency in Multiple Sclerosis Patients and Healthy Controls. Multiple Sclerosis International, 2019, 1-13. https://doi.org/10.1155/2019/2657902

Ahlgren, C., Lycke, J., Odén, A., \& Andersen, O. (2010). High risk of MS in Iranian immigrants in Gothenburg, Sweden. Multiple Sclerosis, 16(9), 1079-1082. https://doi.org/10.1177/1352458510376777

Ahlgren, C., Odén, A., \& Lycke, J. (2012). A nationwide survey of the prevalence of multiple sclerosis in immigrant populations of Sweden. Multiple Sclerosis Journal, 18(8), 1099-1107. https://doi.org/10.1177/1352458511433062

Albert, M., Antel, J., Brück, W., \& Stadelmann, C. (2007). Extensive cortical remyelination in patients with chronic multiple sclerosis. Brain Pathology, 17(2), 129-138. https://doi.org/10.1111/j.1750-3639.2006.00043.x

Alfredsson, L., \& Olsson, T. (2019). Lifestyle and environmental factors in multiple sclerosis. Cold Spring Harbor Perspectives in Medicine, 9(4), a028944. https://doi.org/10.1101/cshperspect.a028944

Amin, M., \& Ontaneda, D. (2021). Thalamic Injury and Cognition in Multiple Sclerosis. Frontiers 
in Neurology, 11. https://doi.org/10.3389/fneur.2020.623914

Anderson, V. M., Fisniku, L. K., Khaleeli, Z., Summers, M. M., Penny, S. A., Altmann, D. R., Thompson, A. J., Ron, M. A., \& Miller, D. H. (2010). Hippocampal atrophy in relapsingremitting and primary progressive MS: A comparative study. Multiple Sclerosis, 16(9), 10831090. https://doi.org/10.1177/1352458510374893

Androdias, G., Reynolds, R., Chanal, M., Ritleng, C., Confavreux, C., \& Nataf, S. (2010). Meningeal T cells associate with diffuse axonal loss in multiple sclerosis spinal cords. Annals of Neurology, 68(4), 465-476. https://doi.org/10.1002/ana.22054

Barkhof, F. (1999). MRI in multiple sclerosis: Correlation with expanded disability status scale (EDSS). Multiple Sclerosis, 5(4), 283-286. https://doi.org/10.1177/135245859900500415

Barkhof, F. (2002). The clinico-radiological paradox in multiple sclerosis revisited. Current Opinion in Neurology, 15(3), 239-245. https://doi.org/10.1097/00019052-200206000-00003

Barkhof, F., Elton, M., Lindeboom, J., Tas, M. W., Schmidt, W. F., Hommes, O. R., Polman, C. H., Kok, A., \& Valk, J. (1998). Functional correlates of callosal atrophy in relapsing-remitting multiple sclerosis patients. A preliminary MRI study. Journal of Neurology, 245(3), 153-158. https://doi.org/10.1007/s004150050196

Barkhof, F., Filippi, M., Miller, D. H., Scheltens, P., Campi, A., Polman, C. H., Comi, G., Adèr, H. J., Losseff, N., \& Valk, J. (1997). Comparison of MRI criteria at first presentation to predict conversion to clinically definite multiple sclerosis. Brain, 120(11), 2059-2069. https://doi.org/10.1093/brain/120.11.2059

Basile, B., Castelli, M., Monteleone, F., Nocentini, U., Caltagirone, C., Centonze, D., Cercignani, M., \& Bozzali, M. (2014). Functional connectivity changes within specific networks parallel the clinical evolution of multiple sclerosis. Multiple Sclerosis Journal, 20(8), 1050-1057. https://doi.org/10.1177/1352458513515082

Bassett, D. S., \& Sporns, O. (2017). Network neuroscience. Nature Neuroscience, 20(3), 353-364. https://doi.org/10.1038/nn.4502

Belbasis, L., Bellou, V., Evangelou, E., Ioannidis, J. P. A., \& Tzoulaki, I. (2015). Environmental risk factors and multiple sclerosis: An umbrella review of systematic reviews and metaanalyses. The Lancet Neurology, 14(3), 263-273. https://doi.org/10.1016/S14744422(14)70267-4

Benedict, R. H. B., Cookfair, D., Gavett, R., Gunther, M., Munschauer, F., Garg, N., \& WeinstockGuttman, B. (2006). Validity of the minimal assessment of cognitive function in multiple sclerosis (MACFIMS). Journal of the International Neuropsychological Society, 12(4), 549558. https://doi.org/10.1017/S1355617706060723

Benedict, R. H. B., Fischer, J. S., Archibald, C. J., Arnett, P. A., Beatty, W. W., Bobholz, J., Chelune, G. J., Fisk, J. D., Langdon, D. W., Caruso, L., Foley, F., LaRocca, N. G., Vowels, L., Weinstein, A., DeLuca, J., Rao, S. M., \& Munschauer, F. (2002). Minimal neuropsychological assessment of MS patients: A consensus approach. Clinical Neuropsychologist, 16(3), 381397. https://doi.org/10.1076/clin.16.3.381.13859

Benedict, R. H. B., Ramasamy, D., Munschauer, F., Weinstock-Guttman, B., \& Zivadinov, R. (2009). Memory impairment in multiple sclerosis: Correlation with deep grey matter and mesial temporal atrophy. Journal of Neurology, Neurosurgery and Psychiatry, 80(2), 201206. https://doi.org/10.1136/jnnp.2008.148403 
Benedict, R. H. B., Weinstock-Guttman, B., Fishman, I., Sharma, J., Tjoa, C. W., \& Bakshi, R. (2004). Prediction of Neuropsychological Impairment in Multiple Sclerosis: Comparison of Conventional Magnetic Resonance Imaging Measures of Atrophy and Lesion Burden. Archives of Neurology, 61(2), 226-230. https://doi.org/10.1001/archneur.61.2.226

Benedict, R. H. B., \& Zivadinov, R. (2011). Risk factors for and management of cognitive dysfunction in multiple sclerosis. Nature Reviews Neurology, 7(6), 332-342. https://doi.org/10.1038/nrneurol.2011.61

Benjamini, Y., \& Hochberg, Y. (1995). Controlling the False Discovery Rate: A Practical and Powerful Approach to Multiple Testing. Journal of the Royal Statistical Society: Series B (Methodological), 57(1), 289-300. https://doi.org/10.1111/j.2517-6161.1995.tb02031.x

Berg-Hansen, P., Moen, S. M., Sandvik, L., Harbo, H. F., Bakken, I. J., Stoltenberg, C., \& Celius, E. G. (2015). Prevalence of multiple sclerosis among immigrants in Norway. Multiple Sclerosis, 21(6), 695-702. https://doi.org/10.1177/1352458514554055

Bergendal, G., Fredrikson, S., \& Almkvist, O. (2007). Selective decline in information processing in subgroups of multiple sclerosis: An 8-year longitudinal study. European Neurology, 57(4), 193-202. https://doi.org/10.1159/000099158

Bergendal, G., Martola, J., Stawiarz, L., Kristoffersen-Wiberg, M., Fredrikson, S., \& Almkvist, O. (2013). Callosal atrophy in multiple sclerosis is related to cognitive speed. Acta Neurologica Scandinavica, 127(4), 281-289. https://doi.org/10.1111/ane.12006

Bergsland, N., Zivadinov, R., Dwyer, M. G., Weinstock-Guttman, B., \& Benedict, R. H. B. (2016). Localized atrophy of the thalamus and slowed cognitive processing speed in MS patients. Multiple Sclerosis, 22(10), 1327-1336. https://doi.org/10.1177/1352458515616204

Bisecco, A., Stamenova, S., Caiazzo, G., d'Ambrosio, A., Sacco, R., Docimo, R., Esposito, S., Cirillo, M., Esposito, F., Bonavita, S., Tedeschi, G., \& Gallo, A. (2018). Attention and processing speed performance in multiple sclerosis is mostly related to thalamic volume. Brain Imaging and Behavior, 12(1), 20-28. https://doi.org/10.1007/s11682-016-9667-6

Bishop, M., \& Rumrill, P. D. (2015). Multiple sclerosis: Etiology, symptoms, incidence and prevalence, and implications for community living and employment. Work, 52(4), 725-734. https://doi.org/10.3233/WOR-152200

Bjørnevik, K., Riise, T., Casetta, I., Drulovic, J., Granieri, E., Holmøy, T., Kampman, M. T., Landtblom, A. M., Lauer, K., Lossius, A., Magalhaes, S., Myhr, K. M., Pekmezovic, T., Wesnes, K., Wolfson, C., \& Pugliatti, M. (2014). Sun exposure and multiple sclerosis risk in Norway and Italy: The EnvIMS study. Multiple Sclerosis Journal, 20(8), 1042-1049. https://doi.org/10.1177/1352458513513968

Bo, L., Geurts, J. J. G., Mork, S. J., \& Valk, P. (2006). Grey matter pathology in multiple sclerosis. Acta Neurologica Scandinavica, 113(s183), 48-50. https://doi.org/10.1111/j.16000404.2006.00615.x

Bø, L., Vedeler, C. A., Nyland, H. I., Trapp, B. D., \& Mørk, S. J. (2003). Subpial demyelination in the cerebral cortex of multiple sclerosis patients. Journal of Neuropathology and Experimental Neurology, 62(7), 723-732. https://doi.org/10.1093/jnen/62.7.723

Bobholz, J. A., \& Gremley, S. (2011). Multiple Sclerosis and Other Demyelinating Disorders. In The Little Black Book of Neuropsychology (pp. 647-661). Springer US. https://doi.org/10.1007/978-0-387-76978-3_20 
Bonavita, S., Sacco, R., Della Corte, M., Esposito, S., Sparaco, M., d'ambrosio, A., Docimo, R., Bisecco, A., Lavorgna, L., Corbo, D., Cirillo, S., Gallo, A., Esposito, F., \& Tedeschi, G.

(2015). Computer-aided cognitive rehabilitation improves cognitive performances and induces brain functional connectivity changes in relapsing remitting multiple sclerosis patients: An exploratory study. Journal of Neurology, 262(1), 91-100. https://doi.org/10.1007/s00415-014$7528-\mathrm{z}$

Bonnet, M. C., Allard, M., Dilharreguy, B., Deloire, M., Petry, K. G., \& Brochet, B. (2010). Cognitive compensation failure in multiple sclerosis. Neurology, 75(14), 1241-1248. https://doi.org/10.1212/WNL.0b013e3181f612e3

Bonzano, L., Pedullà, L., Pardini, M., Tacchino, A., Zaratin, P., Battaglia, M. A., Brichetto, G., \& Bove, M. (2018). Brain activity pattern changes after adaptive working memory training in multiple sclerosis. Brain Imaging and Behavior, 14(1), 142-154. https://doi.org/10.1007/s11682-018-9984-Z

Bonzano, L., Tacchino, A., Brichetto, G., Roccatagliata, L., Dessypris, A., Feraco, P., Lopes De Carvalho, M. L., Battaglia, M. A., Mancardi, G. L., \& Bove, M. (2014). Upper limb motor rehabilitation impacts white matter microstructure in multiple sclerosis. NeuroImage, 90, 107116. https://doi.org/10.1016/j.neuroimage.2013.12.025

Brain, W. R. (1930). Critical review: Disseminated sclerosis. QJM, os-23(91), 343-391. https://doi.org/10.1093/qjmed/os-23.91.343

Brodin, P., Jojic, V., Gao, T., Bhattacharya, S., Angel, C. J. L., Furman, D., Shen-Orr, S., Dekker, C. L., Swan, G. E., Butte, A. J., Maecker, H. T., \& Davis, M. M. (2015). Variation in the human immune system is largely driven by non-heritable influences. Cell, 160(1-2), 37-47. https://doi.org/10.1016/j.cell.2014.12.020

Browne, P., Chandraratna, D., Angood, C., Tremlett, H., Baker, C., Taylor, B. V., \& Thompson, A. J. (2014). Atlas of multiple sclerosis 2013: A growing global problem with widespread inequity. In Neurology (Vol. 83, Issue 11, pp. 1022-1024). Lippincott Williams and Wilkins. https://doi.org/10.1212/WNL.0000000000000768

Buschkuehl, M., Jaeggi, S. M., \& Jonides, J. (2012). Neuronal effects following working memory training. Developmental Cognitive Neuroscience, 2, S167-S179. https://doi.org/10.1016/J.DCN.2011.10.001

Calabrese, M., Favaretto, A., Martini, V., \& Gallo, P. (2013). Grey matter lesions in MS From histology to clinical implications. Prion, 7(1), 20-27. https://doi.org/10.4161/pri.22580

Calabrese, M., Magliozzi, R., Ciccarelli, O., Geurts, J. J. G., Reynolds, R., \& Martin, R. (2015). Exploring the origins of grey matter damage in multiple sclerosis. Nature Reviews Neuroscience, 16(3), 147-158. https://doi.org/10.1038/nrn3900

Calabrese, M., Poretto, V., Favaretto, A., Alessio, S., Bernardi, V., Romualdi, C., Rinaldi, F., Perini, P., \& Gallo, P. (2012). Cortical lesion load associates with progression of disability in multiple sclerosis. Brain, 135(10), 2952-2961. https://doi.org/10.1093/brain/aws246

Calabrese, M., Rinaldi, F., Grossi, P., Mattisi, I., Bernardi, V., Favaretto, A., Perini, P., \& Gallo, P. (2010). Basal ganglia and frontal/parietal cortical atrophy is associated with fatigue in relapsing-remitting multiple sclerosis. Multiple Sclerosis, 16(10), 1220-1228. https://doi.org/10.1177/1352458510376405

Calabrese, M., Rinaldi, F., Mattisi, I., Grossi, P., Favaretto, A., Atzori, M., Bernardi, V., Barachino, 
L., Romualdi, C., Rinaldi, L., Perini, P., \& Gallo, P. (2010). Widespread cortical thinning characterizes patients with MS with mild cognitive impairment. Neurology, 74(4), 321-328. https://doi.org/10.1212/WNL.0b013e3181cbcd03

Calabrese, P., \& Penner, I. K. (2007). Cognitive dysfunctions in multiple sclerosis - A "multiple disconnection syndrome"? In Journal of Neurology (Vol. 254, Issue 2 SUPPL.). Dr. Dietrich Steinkopff Verlag GmbH and Co. KG. https://doi.org/10.1007/s00415-007-2006-5

Camp, S. J., Stevenson, V. L., Thompson, A. J., Miller, D. H., Borras, C., Auriacombe, S., Brochet, B., Falautano, M., Filippi, M., Hérissé-Dulo, L., Montalban, X., Parrcira, E., Polman, C. H., De Sa, J., \& Langdon, D. W. (1999). Cognitive function in primary progressive and transitional progressive multiple sclerosis. A controlled study with MRI correlates. Brain, 122(7), 1341-1348. https://doi.org/10.1093/brain/122.7.1341

Campbell, J., Langdon, D., Cercignani, M., \& Rashid, W. (2016). A Randomised controlled trial of efficacy of cognitive rehabilitation in multiple sclerosis: A cognitive, behavioural, and MRI study. Neural Plasticity, 2016. https://doi.org/10.1155/2016/4292585

Carter, R. E., Lubinsky, J., \& Domholdt, E. (2015). Rehabilitation research. Principles and applications. (Saunders (ed.); 4th Ed).

Castellazzi, G., Debernard, L., Melzer, T. R., Dalrymple-Alford, J. C., D’Angelo, E., Miller, D. H., Gandini Wheeler-Kingshott, C. A. M., \& Mason, D. F. (2018). Functional Connectivity Alterations Reveal Complex Mechanisms Based on Clinical and Radiological Status in Mild Relapsing Remitting Multiple Sclerosis. Frontiers in Neurology, 9(AUG), 690. https://doi.org/10.3389/fneur.2018.00690

Cavanna, A. E., \& Trimble, M. R. (2006). The precuneus: a review of its functional anatomy and behavioural correlates. Brain : A Journal of Neurology, 129(Pt 3), 564-583. https://doi.org/10.1093/brain/aw1004

Cerasa, A., Gioia, M. C., Valentino, P., Nisticò, R., Chiriaco, C., Pirritano, D., Tomaiuolo, F., Mangone, G., Trotta, M., Talarico, T., Bilotti, G., \& Quattrone, A. (2013). Computer-assisted cognitive rehabilitation of attention deficits for multiple sclerosis: A randomized trial with fMRI correlates. Neurorehabilitation and Neural Repair, 27(4), 284-295. https://doi.org/10.1177/1545968312465194

Chao, M. J., Ramagopalan, S. V., Herrera, B. M., Lincoln, M. R., Dyment, D. A., Sadovnick, A. D., \& Ebers, G. C. (2009). Epigenetics in multiple sclerosis susceptibility: Difference in transgenerational risk localizes to the major histocompatibility complex. Human Molecular Genetics, 18(2), 261-266. https://doi.org/10.1093/hmg/ddn353

Charcot, J. (1868). Manuscrits des leçons de JM Charcot. In Fonds numérisé Charcot. Bibliothèque de l'Université Pierre \& Marie Curie. http://jubilotheque.upmc.fr/subset.html?name=collections\&id=charcot.

Charvet, L. E., Yang, J., Shaw, M. T., Sherman, K., Haider, L., Xu, J., \& Krupp, L. B. (2017). Cognitive function in multiple sclerosis improves with telerehabilitation: Results from a randomized controlled trial. PLoS ONE, 12(5). https://doi.org/10.1371/journal.pone.0177177

Chiaravalloti, N. D., \& DeLuca, J. (2008). Cognitive impairment in multiple sclerosis. The Lancet Neurology, 7(12), 1139-1151. https://doi.org/10.1016/S1474-4422(08)70259-X

Chiaravalloti, N. D., Genova, H. M., \& DeLuca, J. (2015). Cognitive rehabilitation in multiple sclerosis: The role of plasticity. Frontiers in Neurology, 6(APR). 
https://doi.org/10.3389/fneur.2015.00067

Chiaravalloti, N. D., Hillary, F. G., Ricker, J. H., Christodoulou, C., Kalnin, A. J., Liu, W., Steffener, J., \& DeLuca, J. (2005). Cerebral Activation Patterns During Working Memory Performance in Multiple Sclerosis Using fMRI. Journal of Clinical and Experimental Neuropsychology, 27(1), 33-54. https://doi.org/10.1080/138033990513609

Chiaravalloti, N. D., Stojanovic-Radic, J., \& DeLuca, J. (2013). The role of speed versus working memory in predicting learning new information in multiple sclerosis. Journal of Clinical and Experimental Neuropsychology, 35(2), 180-191. https://doi.org/10.1080/13803395.2012.760537

Chiaravalloti, N. D., Wylie, G., Leavitt, V., \& DeLuca, J. (2012). Increased cerebral activation after behavioral treatment for memory deficits in MS. Journal of Neurology, 259(7), 1337-1346. https://doi.org/10.1007/s00415-011-6353-x

Cifelli, A., Arridge, M., Jezzard, P., Esiri, M. M., Palace, J., \& Matthews, P. M. (2002). Thalamic neurodegeneration in multiple sclerosis. Annals of Neurology, 52(5), 650-653. https://doi.org/10.1002/ana.10326

Cohen, J. (1988). Statistical Power Analysis for the Behavioral Sciences. In LEA (Ed.), Statistical Power Analysis for the Behavioral Sciences (2nd Ed).

Compston, A., \& Coles, A. (2008). Multiple sclerosis. In The Lancet (Vol. 372, Issue 9648, pp. 1502-1517). Elsevier. https://doi.org/10.1016/S0140-6736(08)61620-7

Compston, A., McDonald, I., Noseworthy, J., Lassmann, H., Miller, D., Smith, K., Wekerle, H., \& Confavreux, C. (2005). McAlpine's Multiple Sclerosis. In Journal of Chemical Information and Modeling (Vol. 53, Issue 9). https://www.elsevier.com/books/mcalpines-multiplesclerosis/9780443072710

Constantinidis, C., \& Klingberg, T. (2016). The neuroscience of working memory capacity and training. Nature Reviews. Neuroscience, 17(7), 438-449. https://doi.org/10.1038/nrn.2016.43

Cortese, M., Riise, T., Bjørnevik, K., Holmøy, T., Kampman, M. T., Magalhaes, S., Pugliatti, M., Wolfson, C., \& Myhr, K. M. (2015). Timing of use of cod liver oil, a vitamin D source, and multiple sclerosis risk: The EnvIMS study. Multiple Sclerosis, 21(14), 1856-1864. https://doi.org/10.1177/1352458515578770

Costa-Arpín, E., Naveiro-Soneira, J., Lema-Bouzas, M., González-Quintela, A., \& Prieto-González, J. M. (2020). Epidemiology of multiple sclerosis in Santiago de Compostela (Spain). Acta Neurologica Scandinavica, 142(3), 267-274. https://doi.org/10.1111/ane.13265

Covey, T. J., Shucard, J. L., Benedict, R. H., Weinstock-Guttman, B., \& Shucard, D. W. (2018). Improved cognitive performance and event-related potential changes following working memory training in patients with multiple sclerosis. Multiple Sclerosis Journal - Experimental, Translational and Clinical, 4(1). https://doi.org/10.1177/2055217317747626

Covey, T. J., Zivadinov, R., Shucard, J. L., \& Shucard, D. W. (2011). Information processing speed, neural efficiency, and working memory performance in multiple sclerosis: Differential relationships with structural magnetic resonance imaging. Journal of Clinical and Experimental Neuropsychology, 33(10), 1129-1145. https://doi.org/10.1080/13803395.2011.614597

Cruz-Gómez, Á. J., Ventura-Campos, N., Belenguer, A., Ávila, C., \& Forn, C. (2014). The link between resting-state functional connectivity and cognition in MS patients. Multiple Sclerosis 
Journal, 20(3), 338-348. https://doi.org/10.1177/1352458513495584

Curti, E., Graziuso, S., Tsantes, E., Crisi, G., \& Granella, F. (2018). Correlation between cortical lesions and cognitive impairment in multiple sclerosis. Brain and Behavior, 8(6). https://doi.org/10.1002/brb3.955

Dalton, C. M. (2004). Early development of multiple sclerosis is associated with progressive grey matter atrophy in patients presenting with clinically isolated syndromes. Brain, 127(5), 11011107. https://doi.org/10.1093/brain/awh126

das Nair, R., Martin, K. J., \& Lincoln, N. B. (2016). Memory rehabilitation for people with multiple sclerosis. In Cochrane Database of Systematic Reviews (Vol. 2016, Issue 3). John Wiley and Sons Ltd. https://doi.org/10.1002/14651858.CD008754.pub3

De Giglio, L., De Luca, F., Prosperini, L., Borriello, G., Bianchi, V., Pantano, P., \& Pozzilli, C. (2015). A low-cost cognitive rehabilitation with a commercial video game improves sustained attention and executive functions in multiple sclerosis: A pilot study. Neurorehabilitation and Neural Repair, 29(5), 453-461. https://doi.org/10.1177/1545968314554623

De Giglio, L., Tona, F., De Luca, F., Petsas, N., Prosperini, L., Bianchi, V., Pozzilli, C., \& Pantano, P. (2016). Multiple sclerosis: Changes in thalamic resting-State functional connectivity induced by A homebased cognitive rehabilitation program. Radiology, 280(1), 202-211. https://doi.org/10.1148/radiol.2016150710

De Giglio, L., Upadhyay, N., De Luca, F., Prosperini, L., Tona, F., Petsas, N., Pozzilli, C., \& Pantano, P. (2016). Corpus callosum microstructural changes associated with Kawashima Nintendo Brain Training in patients with multiple sclerosis. Journal of the Neurological Sciences, 370, 211-213. https://doi.org/10.1016/j.jns.2016.09.041

Del Giudice, M., Booth, T., \& Irwing, P. (2012). The distance between mars and venus: Measuring global sex differences in personality. PLOS ONE, 7(1). https://doi.org/10.1371/journal.pone.0029265

Deloire, M. S. A., Bonnet, M. C., Salort, E., Arimone, Y., Boudineau, M., Petry, K. G., \& Brochet, B. (2006). How to detect cognitive dysfunction at early stages of multiple sclerosis? Multiple Sclerosis, 12(4), 445-452. https://doi.org/10.1191/1352458506ms1289oa

Deloire, M. S. A., Ruet, A., Hamel, D., Bonnet, M., Dousset, V., \& Brochet, B. (2011). MRI predictors of cognitive outcome in early multiple sclerosis. Neurology, 76(13), 1161-1167. https://doi.org/10.1212/WNL.0b013e318212a8be

Deloire, M. S. A., Salort, E., Bonnet, M., Arimone, Y., Boudineau, M., Amieva, H., Barroso, B., Ouallet, J. C., Pachai, C., Galliaud, E., Petry, K. G., Dousset, V., Fabrigoule, C., \& Brochet, B. (2005). Cognitive impairment as marker of diffuse brain abnormalities in early relapsing remitting multiple sclerosis. Journal of Neurology, Neurosurgery and Psychiatry, 76(4), 519526. https://doi.org/10.1136/jnnp.2004.045872

DeLuca, G. C., Ebers, G. C., \& Esiri, M. M. (2004). Axonal loss in multiple sclerosis: A pathological survey of the corticospinal and sensory tracts. In Brain (Vol. 127, Issue 5, pp. 1009-1018). Brain. https://doi.org/10.1093/brain/awh118

DeLuca, G. C., Yates, R. L., Beale, H., \& Morrow, S. A. (2015). Cognitive Impairment in Multiple Sclerosis: Clinical, Radiologic and Pathologic Insights. Brain Pathology, 25(1), 79-98. https://doi.org/10.1111/bpa.12220

DeLuca, J., Barbieri-Berger, S., \& Johnson, S. K. (1994). The Nature of Memory Impairments in 
Multiple Sclerosis: Acquisition versus Retrieval. Journal of Clinical and Experimental Neuropsychology, 16(2), 183-189. https://doi.org/10.1080/01688639408402629

DeLuca, J., Chelune, G. J., Tulsky, D. S., Lengenfelder, J., \& Chiaravalloti, N. D. (2004). Is speed of processing or working memory the primary information processing deficit in multiple sclerosis? Journal of Clinical and Experimental Neuropsychology, 26(4), 550-562. https://doi.org/10.1080/13803390490496641

Demaree, H. A., DeLuca, J., Gaudino, E. A., \& Diamond, B. J. (1999). Speed of information processing as a key deficit in multiple sclerosis: Implications for rehabilitation. Journal of Neurology Neurosurgery and Psychiatry, 67(5), 661-663. https://doi.org/10.1136/jnnp.67.5.661

Dendrou, C. A., Fugger, L., \& Friese, M. A. (2015). Immunopathology of multiple sclerosis. Nature Reviews Immunology, 15(9), 545-558. https://doi.org/10.1038/nri3871

Denney, D. R., \& Lynch, S. G. (2009). The impact of multiple sclerosis on patients' performance on the Stroop Test: Processing speed versus interference. Journal of the International Neuropsychological Society, 15(3), 451-458. https://doi.org/10.1017/S1355617709090730

Detels, R., Visscher, B. R., Malmgren, R. M., Coulson, A. H., Lucia, M. V., \& Dudley, J. P. (1977). Evidence for lower susceptibility to multiple sclerosis in Japanese-Americans. American Journal of Epidemiology, 105(4), 303-310. https://doi.org/10.1093/oxfordjournals.aje.a112387

Díez-Cirarda, M., Ibarretxe-Bilbao, N., Peña, J., \& Ojeda, N. (2018). Efficacy of cognitive rehabilitation in Parkinson's disease. In Neural Regeneration Research (Vol. 13, Issue 2, pp. 226-227). Wolters Kluwer Medknow Publications. https://doi.org/10.4103/1673-5374.226390

Diez, I., \& Sepulcre, J. (2018). Neurogenetic profiles delineate large-scale connectivity dynamics of the human brain. Nature Communications, 9(1). https://doi.org/10.1038/s41467-018-06346-3

Disanto, G., Berlanga, A. J., Handel, A. E., Para, A. E., Burrell, A. M., Fries, A., Handunnetthi, L., De Luca, G. C., \& Morahan, J. M. (2011). Heterogeneity in multiple sclerosis: Scratching the surface of a complex disease. In Autoimmune Diseases (Vol. 1, Issue 1). https://doi.org/10.4061/2011/932351

Dobbs, A. R., \& Rule, B. G. (1989). Adult age differences in working memory. Psychology and Aging, 4(4), 500-503. https://doi.org/10.1037/0882-7974.4.4.500

Dobryakova, E., Wylie, G. R., DeLuca, J., \& Chiaravalloti, N. D. (2014). A pilot study examining functional brain activity 6 months after memory retraining in MS: the MEMREHAB trial. Brain Imaging and Behavior, 8(3), 403-406. https://doi.org/10.1007/s11682-014-9309-9

Drew, M., Tippett, L. J., Starkey, N. J., \& Isler, R. B. (2008). Executive dysfunction and cognitive impairment in a large community-based sample with Multiple Sclerosis from New Zealand: A descriptive study. Archives of Clinical Neuropsychology, 23(1), 1-19. https://doi.org/10.1016/j.acn.2007.09.005

Droby, A., Yuen, K. S. L., Muthuraman, M., Reitz, S. C., Fleischer, V., Klein, J., Gracien, R. M., Ziemann, U., Deichmann, R., Zipp, F., \& Groppa, S. (2016). Changes in brain functional connectivity patterns are driven by an individual lesion in MS: a resting-state fMRI study. Brain Imaging and Behavior, 10(4), 1117-1126. https://doi.org/10.1007/s11682-015-9476-3

Dutta, R., Chang, A., Doud, M. K., Kidd, G. J., Ribaudo, M. V., Young, E. A., Fox, R. J., Staugaitis, S. M., \& Trapp, B. D. (2011). Demyelination causes synaptic alterations in 
hippocampi from multiple sclerosis patients. Annals of Neurology, 69(3), 445-454. https://doi.org/10.1002/ana.22337

Dziedzic, T., Metz, I., Dallenga, T., König, F. B., Müller, S., Stadelmann, C., \& Brück, W. (2010). Wallerian degeneration: A major component of early axonal pathology in multiple sclerosis. Brain Pathology, 20(5), 976-985. https://doi.org/10.1111/j.1750-3639.2010.00401.x

Ebers, G. C., Yee, I. M. L., Sadovnick, A. D., \& Duquette, P. (2000). Conjugal multiple sclerosis: Population-based prevalence and recurrence risks in offspring. Annals of Neurology, 48(6), 927-931. https://doi.org/10.1002/1531-8249(200012)48:6<927::AID-ANA14>3.0.CO;2-F

Einarsson, U., Gottberg, K., von Koch, L., Fredrikson, S., Ytterberg, C., Jin, Y. P., Andersson, M., \& Holmqvist, L. W. (2006). Cognitive and motor function in people with multiple sclerosis in Stockholm County. Multiple Sclerosis, 12(3), 340-353. https://doi.org/10.1191/135248506ms1259oa

Emch, M., von Bastian, C. C., \& Koch, K. (2019). Neural correlates of verbal working memory: An fMRI meta-analysis. Frontiers in Human Neuroscience, 13(June), 1-17. https://doi.org/10.3389/fnhum.2019.00180

Ernst, A., Blanc, F., Voltzenlogel, V., De Seze, J., Chauvin, B., \& Manning, L. (2013). Autobiographical memory in multiple sclerosis patients: Assessment and cognitive facilitation. Neuropsychological Rehabilitation, 23(2), 161-181. https://doi.org/10.1080/09602011.2012.724355

Ernst, A., Botzung, A., Gounot, D., Sellal, F., Blanc, F., De Seze, J., \& Manning, L. (2012). Induced Brain Plasticity after a Facilitation Programme for Autobiographical Memory in Multiple Sclerosis: A Preliminary Study. Multiple Sclerosis International, 2012, 12. https://doi.org/10.1155/2012/820240

Ernst, A., Sourty, M., Roquet, D., Noblet, V., Gounot, D., Blanc, F., de Seze, J., \& Manning, L. (2016). Benefits from an autobiographical memory facilitation programme in relapsingremitting multiple sclerosis patients: a clinical and neuroimaging study. Neuropsychological Rehabilitation, 28(7), 1110-1130. https://doi.org/10.1080/09602011.2016.1240697

Ernst, A., Sourty, M., Roquet, D., Noblet, V., Gounot, D., Blanc, F., De Seze, J., \& Manning, L. (2016). Functional and structural cerebral changes in key brain regions after a facilitation programme for episodic future thought in relapsing-remitting multiple sclerosis patients. Brain and Cognition, 105, 34-45. https://doi.org/10.1016/J.BANDC.2016.03.007

Evangelou, N., Konz, D., Esiri, M. M., Smith, S., Palace, J., \& Matthews, P. M. (2000). Regional axonal loss in the corpus callosum correlates with cerebral white matter lesion volume and distribution in multiple sclerosis. Brain, 123(9), 1845-1849. https://doi.org/10.1093/brain/123.9.1845

Fawcett, J., \& Skegg, D. (1988). Geographic distribution of MS in new zealand: Evidence from hospital admissions and deaths. Neurology, 38(3), 416-418. https://doi.org/10.1212/wnl.38.3.416

Federation, M. I. (2020). Number of people with MS. https://www.atlasofms.org/chart/unitedkingdom/epidemiology/female-to-male-ratio-of-people-with-ms

Filippi, M., Riccitelli, G., Mattioli, F., Capra, R., Stampatori, C., Pagani, E., Valsasina, P., Copetti, M., Falini, A., Comi, G., \& Rocca, M. A. (2012). Multiple sclerosis: Effects of cognitive rehabilitation on structural and functional MR imaging measures - An explorative study. 
Radiology, 262(3), 932-940. https://doi.org/10.1148/radiol.11111299

Filippi, M., Rocca, M. A., Barkhof, F., Brück, W., Chen, J. T., Comi, G., DeLuca, G., De Stefano, N., Erickson, B. J., Evangelou, N., Fazekas, F., Geurts, J. J. G., Lucchinetti, C., Miller, D. H., Pelletier, D., Popescu, B. F. G., \& Lassmann, H. (2012). Association between pathological and MRI findings in multiple sclerosis. The Lancet Neurology, 11(4), 349-360. https://doi.org/10.1016/S1474-4422(12)70003-0

Filippi, M., Tortorella, C., Rovaris, M., Bozzali, M., Possa, F., Sormani, M. P., Iannucci, G., \& Comi, G. (2000). Changes in the normal appearing brain tissue and cognitive impairment in multiple sclerosis. Journal of Neurology Neurosurgery and Psychiatry, 68(2), 157-161. https://doi.org/10.1136/jnnp.68.2.157

Fittipaldi-Márquez, M. S., Cruz-Gómez, Á. J., Sanchis-Segura, C., Belenguer, A., Ávila, C., \& Forn, C. (2017). Exploring Neural Efficiency in Multiple Sclerosis Patients during the Symbol Digit Modalities Test: A Functional Magnetic Resonance Imaging Study. Neurodegenerative Diseases, 17(4-5), 199-207. https://doi.org/10.1159/000460252

Fleischer, V., Gröger, A., Koirala, N., Droby, A., Muthuraman, M., Kolber, P., Reuter, E., Meuth, S. G., Zipp, F., \& Groppa, S. (2017). Increased structural white and grey matter network connectivity compensates for functional decline in early multiple sclerosis. Multiple Sclerosis, 23(3), 432-441. https://doi.org/10.1177/1352458516651503

Fleischer, V., Radetz, A., Ciolac, D., Muthuraman, M., Gonzalez-Escamilla, G., Zipp, F., \& Groppa, S. (2019). Graph Theoretical Framework of Brain Networks in Multiple Sclerosis: A Review of Concepts. In Neuroscience (Vol. 403, pp. 35-53). Elsevier Ltd. https://doi.org/10.1016/j.neuroscience.2017.10.033

Forn, C. (2020). Neuropsicología de las enfermedades desmielinizantes: esclerosis múltiple. In Pirámide (Ed.), Manual de neuropsicología (1st ed., pp. 195-214). https://www.edicionespiramide.es/libro.php?id=6430644

Forn, C., Barros-Loscertales, A., Escudero, J., Belloch, V., Campos, S., Parcet, M. A., \& Ávila, C. (2006). Cortical reorganization during PASAT task in MS patients with preserved working memory functions. NeuroImage, 31(2), 686-691. https://doi.org/10.1016/j.neuroimage.2005.12.030

Forn, C., Barros-Loscertales, A., Escudero, J., Benlloch, V., Campos, S., Parcet, M., \& Ávila, C. (2007). Compensatory activations in patients with multiple sclerosis during preserved performance on the auditory N-back task. Human Brain Mapping, 28(5), 424-430. https://doi.org/10.1002/hbm.20284

Forn, C., Belenguer, A., Belloch, V., Sanjuan, A., Parcet, M. A., \& Ávila, C. (2011). Anatomical and functional differences between the paced auditory serial addition test and the symbol digit modalities test. Journal of Clinical and Experimental Neuropsychology, 33(1), 42-50. https://doi.org/10.1080/13803395.2010.481620

Forn, C., Belenguer, A., Parcet-Ibars, M. A., \& Avila, C. (2008). Information-processing speed is the primary deficit underlying the poor performance of multiple sclerosis patients in the Paced Auditory Serial Addition Test (PASAT). Journal of Clinical and Experimental Neuropsychology, 30(7), 789-796. https://doi.org/10.1080/13803390701779560

Forn, C., Belloch, V., Bustamante, J. C., Garbin, G., Parcet-Ibars, M. À., Sanjuan, A., Ventura, N., \& Ávila, C. (2009). A Symbol Digit Modalities Test version suitable for functional MRI studies. Neuroscience Letters, 456(1), 11-14. https://doi.org/10.1016/j.neulet.2009.03.081 
Forn, C., Ripollés, P., Cruz-Gómez, A. J., Belenguer, A., González-Torre, J. A., \& Ávila, C. (2013). Task-load manipulation in the Symbol Digit Modalities Test: An alternative measure of information processing speed. Brain and Cognition, 82(2), 152-160. https://doi.org/10.1016/J.BANDC.2013.04.003

Friston, K. J. (1994). Functional and effective connectivity in neuroimaging: A synthesis. Human Brain Mapping, 2(1-2), 56-78. https://doi.org/10.1002/hbm.460020107

Fulton, J. C., Grossman, R. I., Udupa, J., Mannon, L. J., Grossman, M., Wei, L., Polansky, M., \& Kolson, D. L. (1999). MR lesion load and cognitive function in patients with relapsingremitting multiple sclerosis. American Journal of Neuroradiology, 20(10), 1951-1955. /pmc/articles/PMC7657798/

Gale, C. R., \& Martyn, C. N. (1995). Migrant studies in multiple sclerosis. In Progress in Neurobiology (Vol. 47, Issues 4-5, pp. 425-448). Pergamon. https://doi.org/10.1016/03010082(95)80008-V

Gamboa, O. L., Tagliazucchi, E., Von Wegner, F., Jurcoane, A., Wahl, M., Laufs, H., \& Ziemann, U. (2014). Working memory performance of early MS patients correlates inversely with modularity increases in resting state functional connectivity networks. In NeuroImage (Vol. 94, pp. 385-395). Academic Press Inc. https://doi.org/10.1016/j.neuroimage.2013.12.008

Ganter, P., Prince, C., \& Esiri, M. M. (1999). Spinal cord axonal loss in multiple sclerosis: A postmortem study. Neuropathology and Applied Neurobiology, 25(6), 459-467. https://doi.org/10.1046/j.1365-2990.1999.00205.x

Ghadirian, P., Dadgostar, B., Azani, R., \& Maisonneuve, P. (2001). A case-control study of the association between socio-demographic, lifestyle and medical history factors and multiple sclerosis. Canadian Journal of Public Health, 92(4), 281-285. https://doi.org/10.1007/bf03404961

Gianfrancesco, M. A., Stridh, P., Rhead, B., Shao, X., Xu, E., Graves, J. S., Chitnis, T., Waldman, A., Lotze, T., Schreiner, T., Belman, A., Greenberg, B., Weinstock-Guttman, B., Aaen, G., Tillema, J. M., Hart, J., Caillier, S., Ness, J., Harris, Y., ... Waubant, E. (2017). Evidence for a causal relationship between low Vitamin D, high BMI, and pediatric-onset MS. Neurology, 88(17), 1623-1629. https://doi.org/10.1212/WNL.0000000000003849

González Torre, J. A., Cruz-Gómez, Á. J., Belenguer, A., Sanchis-Segura, C., Ávila, C., \& Forn, C. (2017). Hippocampal dysfunction is associated with memory impairment in multiple sclerosis: A volumetric and functional connectivity study. Multiple Sclerosis, 23(14), 1854-1863. https://doi.org/10.1177/1352458516688349

Goverover, Y., Chiaravalloti, N. D., O’Brien, A. R., \& DeLuca, J. (2018). Evidenced-Based Cognitive Rehabilitation for Persons With Multiple Sclerosis: An Updated Review of the Literature From 2007 to 2016. In Archives of Physical Medicine and Rehabilitation (Vol. 99, Issue 2, pp. 390-407). W.B. Saunders. https://doi.org/10.1016/j.apmr.2017.07.021

Granberg, T., Martola, J., Bergendal, G., Shams, S., Damangir, S., Aspelin, P., Fredrikson, S., \& Kristoffersen-Wiberg, M. (2015). Corpus callosum atrophy is strongly associated with cognitive impairment in multiple sclerosis: Results of a 17-year longitudinal study. Multiple Sclerosis, 21(9), 1151-1158. https://doi.org/10.1177/1352458514560928

Grønlie, S. A., Myrvoll, E., Hansen, G., Grønning, M., \& Mellgren, S. I. (2000). Multiple sclerosis in North Norway, and first appearance in an indigenous population. Journal of Neurology, 247(2), 129-133. https://doi.org/10.1007/PL00007793 
Gross, H. J., \& Watson, C. (2017). Characteristics, burden of illness, and physical functioning of patients with relapsing-remitting and secondary progressive multiple sclerosis: A crosssectional US survey. Neuropsychiatric Disease and Treatment, 13, 1349-1357. https://doi.org/10.2147/NDT.S132079

Grzegorski, T., \& Losy, J. (2017). Cognitive impairment in multiple sclerosis - A review of current knowledge and recent research. In Reviews in the Neurosciences (Vol. 28, Issue 8, pp. 845860). Walter de Gruyter GmbH. https://doi.org/10.1515/revneuro-2017-0011

Guimarães, J., \& Sá, M. J. (2012). Cognitive dysfunction in Multiple Sclerosis. Frontiers in Neurology, MAY. https://doi.org/10.3389/fneur.2012.00074

Haider, L., Zrzavy, T., Hametner, S., Höftberger, R., Bagnato, F., Grabner, G., Trattnig, S., Pfeifenbring, S., Brück, W., \& Lassmann, H. (2016). The topograpy of demyelination and neurodegeneration in the multiple sclerosis brain. Brain, 139(3), 807-815. https://doi.org/10.1093/brain/awv398

Hampshire, A., Chamberlain, S. R., Monti, M. M., Duncan, J., \& Owen, A. M. (2010). The role of the right inferior frontal gyrus: inhibition and attentional control. NeuroImage, 50(3), 13131319. https://doi.org/10.1016/j.neuroimage.2009.12.109

Hancock, L. M., Bruce, J. M., Bruce, A. S., \& Lynch, S. G. (2015). Processing speed and working memory training in multiple sclerosis: A double-blind randomized controlled pilot study. Journal of Clinical and Experimental Neuropsychology, 37(2), 113-127. https://doi.org/10.1080/13803395.2014.989818

Handel, A. E., Williamson, A. J., Disanto, G., Handunnetthi, L., Giovannoni, G., \& Ramagopalan, S. V. (2010). An Updated Meta-Analysis of Risk of Multiple Sclerosis following Infectious Mononucleosis. PLoS ONE, 5(9), e12496. https://doi.org/10.1371/journal.pone.0012496

Hawellek, D. J., Hipp, J. F., Lewis, C. M., Corbetta, M., \& Engel, A. K. (2011). Increased functional connectivity indicates the severity of cognitive impairment in multiple sclerosis. Proceedings of the National Academy of Sciences of the United States of America, 108(47), 19066-19071. https://doi.org/10.1073/pnas.1110024108

Hedström, A. K., Bäärnhielm, M., Olsson, T., \& Alfredsson, L. (2009). Tobacco smoking, but not Swedish snuff use, increases the risk of multiple sclerosis. Neurology, 73(9), 696-701. https://doi.org/10.1212/WNL.0b013e3181b59c40

Hedström, A. K., Bäärnhielm, M., Olsson, T., \& Alfredsson, L. (2011). Exposure to environmental tobacco smoke is associated with increased risk for multiple sclerosis. Multiple Sclerosis Journal, 17(7), 788-793. https://doi.org/10.1177/1352458511399610

Hedström, A. K., Olsson, T., \& Alfredsson, L. (2016). Body mass index during adolescence, rather than childhood, is critical in determining MS risk. Multiple Sclerosis, 22(7), 878-883. https://doi.org/10.1177/1352458515603798

Heesen, C., Schulz, K. H., Fiehler, J., Von der Mark, U., Otte, C., Jung, R., Poettgen, J., Krieger, T., \& Gold, S. M. (2010). Correlates of cognitive dysfunction in multiple sclerosis. Brain, Behavior, and Immunity, 24(7), 1148-1155. https://doi.org/10.1016/j.bbi.2010.05.006

Henry, J. D., \& Beatty, W. W. (2006). Verbal fluency deficits in multiple sclerosis. Neuropsychologia, 44(7), 1166-1174. https://doi.org/10.1016/j.neuropsychologia.2005.10.006

Heydarpour, P., Amini, H., Khoshkish, S., Seidkhani, H., Sahraian, M. A., \& Yunesian, M. (2014). Potential impact of air pollution on multiple sclerosis in Tehran, Iran. Neuroepidemiology, 
43(3-4), 233-238. https://doi.org/10.1159/000368553

Houtchens, M. K., Benedict, R. H. B., Killiany, R., Sharma, J., Jaisani, Z., Singh, B., WeinstockGuttman, B., Guttmann, C. R. G., \& Bakshi, R. (2007). Thalamic atrophy and cognition in multiple sclerosis. Neurology, 69(12), 1213-1223. https://doi.org/10.1212/01.wnl.0000276992.17011.b5

Hubacher, M., DeLuca, J., Weber, P., Steinlin, M., Kappos, L., Opwis, K., \& Penner, I. K. (2015). Cognitive rehabilitation of working memory in juvenile multiple sclerosis-effects on cognitive functioning, functional MRI and network related connectivity. Restorative Neurology and Neuroscience, 33(5), 713-725. https://doi.org/10.3233/RNN-150497

Hubacher, M., Kappos, L., Weier, K., Stöcklin, M., Opwis, K., \& Penner, I.-K. (2015). Case-Based fMRI Analysis after Cognitive Rehabilitation in MS: A Novel Approach. Frontiers in Neurology, 6(MAR), 78. https://doi.org/10.3389/fneur.2015.00078

Hughes, A. J., Denney, D. R., \& Lynch, S. G. (2011). Reaction time and rapid serial processing measures of information processing speed in multiple sclerosis: Complexity, compounding, and augmentation. Journal of the International Neuropsychological Society, 17(6), 11131121. https://doi.org/10.1017/S1355617711001135

Huiskamp, M., Dobryakova, E., Wylie, G. D., Deluca, J., \& Chiaravalloti, N. D. (2016). A pilot study of changes in functional brain activity during a working memory task after mSMT treatment: The MEMREHAB trial. Multiple Sclerosis and Related Disorders, 7, 76-82. https://doi.org/10.1016/j.msard.2016.03.012

Hulst, H. E., Gehring, K., Uitdehaag, B. M. J., Visser, L. H., Polman, C. H., Barkhof, F., Sitskoorn, M. M., \& Geurts, J. J. G. (2014). Indicators for cognitive performance and subjective cognitive complaints in multiple sclerosis: A role for advanced MRI. Multiple Sclerosis Journal, 20(8), 1131-1134. https://doi.org/10.1177/1352458513513969

Hulst, H. E., Schoonheim, M. M., Van Geest, Q., Uitdehaag, B. M. J., Barkhof, F., \& Geurts, J. J. G. (2015). Memory impairment in multiple sclerosis: Relevance of hippocampal activation and hippocampal connectivity. Multiple Sclerosis Journal, 21(13), 1705-1712. https://doi.org/10.1177/1352458514567727

Hulst, H. E., Steenwijk, M. D., Versteeg, A., Pouwels, P. J. W., Vrenken, H., Uitdehaag, B. M. J., Polman, C. H., Geurts, J. J. G., \& Barkhof, F. (2013). Cognitive impairment in MS: Impact of white matter integrity, gray matter volume, and lesions. Neurology, 80(11), 1025-1032. https://doi.org/10.1212/WNL.0b013e31828726cc

Islas, M. Á. M., \& Ciampi, E. (2019). Assessment and impact of cognitive impairment in multiple sclerosis: An overview. In Biomedicines (Vol. 7, Issue 1). MDPI AG. https://doi.org/10.3390/biomedicines7010022

Jacola, L. M., Willard, V. W., Ashford, J. M., Ogg, R. J., Scoggins, M. A., Jones, M. M., Wu, S., \& Conklin, H. M. (2014). Clinical utility of the N-back task in functional neuroimaging studies of working memory. Journal of Clinical and Experimental Neuropsychology, 36(8), 875-886. https://doi.org/10.1080/13803395.2014.953039

Jaeggi, S. M., Buschkuehl, M., Jonides, J., \& Perrig, W. J. (2008). Improving fluid intelligence with training on working memory. Proceedings of the National Academy of Sciences of the United States of America, 105(19), 6829-6833. https://doi.org/10.1073/pnas.0801268105

Jaeggi, S. M., Buschkuehl, M., Perrig, W. J., \& Meier, B. (2010). The concurrent validity of the N- 
back task as a working memory measure. Memory, 18(4), 394-412.

https://doi.org/10.1080/09658211003702171

Janculjak, D., Mubrin, Z., Brinar, V., \& Spilich, G. (2002). Changes of attention and memory in a group of patients with multiple sclerosis. Clinical Neurology and Neurosurgery, 104(3), 221227. https://doi.org/10.1016/S0303-8467(02)00042-2

Jaroudi, W., Garami, J., Garrido, S., Hornberger, M., Keri, S., \& Moustafa, A. A. (2017). Factors underlying cognitive decline in old age and Alzheimer's disease: The role of the hippocampus. Reviews in the Neurosciences, 28(7), 705-714. https://doi.org/10.1515/revneuro-2016-0086

Jersild, C., Dupont, B., Fog, T., Hansen, G. S., Nielsen, L. S., Thomsen, M., \& Svejgaard, A. (1973). Histocompatibility-linked immune-response determinants in multiple sclerosis. Transplantation Proceedings, 5(4), 1791-1796.

Juha, M., Leszek, S., Sten, F., Jan, H., Jakob, B., Olof, F., \& Maria, K. W. (2007). Progression of non-age-related callosal brain atrophy in multiple sclerosis: A 9-year longitudinal MRI study representing four decades of disease development. Journal of Neurology, Neurosurgery and Psychiatry, 78(4), 375-380. https://doi.org/10.1136/jnnp.2006.106690

Kampman, M. T., Wilsgaard, T., \& Mellgren, S. I. (2007). Outdoor activities and diet in childhood and adolescence relate to MS risk above the Arctic Circle. Journal of Neurology, 254(4), 471477. https://doi.org/10.1007/s00415-006-0395-5

Kassambara, A. (2020). rstatix: Pipe-Friendly Framework for Basic Statistical Tests. R package version 0.4.0. https://cran.r-project.org/package=rstatix

Kidd, D., Barkhof, F., McConnell, R., Algra, P. R., Allen, I. V., \& Revesz, T. (1999). Cortical lesions in multiple sclerosis. Brain, 122(1), 17-26. https://doi.org/10.1093/brain/122.1.17

Koch-Henriksen, N., \& Sørensen, P. S. (2010). The changing demographic pattern of multiple sclerosis epidemiology. In The Lancet Neurology (Vol. 9, Issue 5, pp. 520-532). Lancet Neurol. https://doi.org/10.1016/S1474-4422(10)70064-8

Kujala, P., Portin, R., Revonsuo, A., \& Ruutiainen, J. (1995). Attention related performance in two cognitively different subgroups of patients with multiple sclerosis. Journal of Neurology Neurosurgery and Psychiatry, 59(1), 77-82. https://doi.org/10.1136/jnnp.59.1.77

Kutzelnigg, A., Lucchinetti, C. F., Stadelmann, C., Brück, W., Rauschka, H., Bergmann, M., Schmidbauer, M., Parisi, J. E., \& Lassmann, H. (2005). Cortical demyelination and diffuse white matter injury in multiple sclerosis. Brain, 128(11), 2705-2712. https://doi.org/10.1093/brain/awh641

Lampit, A., Heine, J., Finke, C., Barnett, M. H., Valenzuela, M., Wolf, A., Leung, I. H. K., \& Hill, N. T. M. (2019). Computerized Cognitive Training in Multiple Sclerosis: A Systematic Review and Meta-analysis. In Neurorehabilitation and Neural Repair (Vol. 33, Issue 9, pp. 695-706). SAGE Publications Inc. https://doi.org/10.1177/1545968319860490

Langdon, D. W. (2011). Cognition in multiple sclerosis. Current Opinion in Neurology, 24(3), 244249. https://doi.org/10.1097/WCO.0b013e328346a43b

Langdon, D. W., Amato, M. P., Boringa, J., Brochet, B., Foley, F., Fredrikson, S., Hämäläinen, P., Hartung, H. P., Krupp, L., Penner, I. K., Reder, A. T., \& Benedict, R. H. B. (2012).

Recommendations for a brief international cognitive assessment for multiple sclerosis (BICAMS). In Multiple Sclerosis Journal (Vol. 18, Issue 6, pp. 891-898). SAGE Publications. https://doi.org/10.1177/1352458511431076 
Langer-Gould, A., Brara, S. M., Beaber, B. E., \& Koebnick, C. (2013). Childhood obesity and risk of pediatric multiple sclerosis and clinically isolated syndrome. Neurology, 80(6), 548-552. https://doi.org/10.1212/WNL.0b013e31828154f3

Lansley, J., Mataix-Cols, D., Grau, M., Radua, J., \& Sastre-Garriga, J. (2013). Localized grey matter atrophy in multiple sclerosis: A meta-analysis of voxel-based morphometry studies and associations with functional disability. In Neuroscience and Biobehavioral Reviews (Vol. 37, Issue 5, pp. 819-830). Pergamon. https://doi.org/10.1016/j.neubiorev.2013.03.006

Lassmann, H. (2018). Multiple sclerosis pathology. In Cold Spring Harbor Perspectives in Medicine (Vol. 8, Issue 3, p. a028936). Cold Spring Harbor Laboratory Press. https://doi.org/10.1101/cshperspect.a028936

Lazeron, R. H. C., de Sonneville, L. M. J., Scheltens, P., Polman, C. H., \& Barkhof, F. (2006). Cognitive slowing in multiple sclerosis is strongly associated with brain volume reduction. Multiple Sclerosis, 12(6), 760-768. https://doi.org/10.1177/1352458506070924

Leavitt, V. M., Lengenfelder, J., Moore, N. B., Chiaravalloti, N. D., \& DeLuca, J. (2011). The relative contributions of processing speed and cognitive load to working memory accuracy in multiple sclerosis. Journal of Clinical and Experimental Neuropsychology, 33(5), 580-586. https://doi.org/10.1080/13803395.2010.541427

Leavitt, V. M., Wylie, G., Krch, D., Chiaravalloti, N., DeLuca, J., \& Sumowski, J. F. (2014). Does slowed processing speed account for executive deficits in multiple sclerosis? Evidence from neuropsychological performance and structural neuroimaging. Rehabilitation Psychology, 59(4), 422-428. https://doi.org/10.1037/a0037517

Leavitt, V. M., Wylie, G. R., Girgis, P. A., DeLuca, J., \& Chiaravalloti, N. D. (2014). Increased functional connectivity within memory networks following memory rehabilitation in multiple sclerosis. Brain Imaging and Behavior, 8(3), 394-402. https://doi.org/10.1007/s11682-0129183-2

Lee, J. Y., Taghian, K., \& Petratos, S. (2014). Axonal degeneration in multiple sclerosis: Can we predict and prevent permanent disability? In Acta Neuropathologica Communications (Vol. 2, Issue 1). BioMed Central Ltd. https://doi.org/10.1186/s40478-014-0097-7

Lengenfelder, J., Bryant, D., Diamond, B. J., Kalmar, J. H., Moore, N. B., \& DeLuca, J. (2006). Processing speed interacts with working memory efficiency in multiple sclerosis. Archives of Clinical Neuropsychology, 21(3), 229-238. https://doi.org/10.1016/j.acn.2005.12.001

Lengenfelder, J., Chiaravalloti, N. D., Ricker, J. H., \& DeLuca, J. (2003). Deciphering components of impaired working memory in multiple sclerosis. Cognitive and Behavioral Neurology, 16(1), 28-39. https://doi.org/10.1097/00146965-200303000-00004

Leray, E., Moreau, T., Fromont, A., \& Edan, G. (2016). Epidemiology of multiple sclerosis. Revue Neurologique, 172(1), 3-13. https://doi.org/10.1016/j.neurol.2015.10.006

Levy, B. J., \& Wagner, A. D. (2011). Cognitive control and right ventrolateral prefrontal cortex: Reflexive reorienting, motor inhibition, and action updating. Annals of the New York Academy of Sciences, 1224(1), 40-62. https://doi.org/10.1111/j.1749-6632.2011.05958.x

Litvan, I., Grafman, J., Vendrell, P., Martinez, J. M., Junqué, C., Vendrell, J. M., \& Barraquer Bordas, J. L. (1988). Multiple Memory Deficits in Patients with Multiple Sclerosis: Exploring the Working Memory System. Archives of Neurology, 45(6), 607-610. https://doi.org/10.1001/archneur.1988.00520300025012 
Louapre, C., Perlbarg, V., García-Lorenzo, D., Urbanski, M., Benali, H., Assouad, R., Galanaud, D., Freeman, L., Bodini, B., Papeix, C., Tourbah, A., Lubetzki, C., Lehéricy, S., \& Stankoff, B. (2014). Brain networks disconnection in early multiple sclerosis cognitive deficits: An anatomofunctional study. Human Brain Mapping, 35(9), 4706-4717. https://doi.org/10.1002/hbm.22505

Lucas, R. M., Byrne, S. N., Correale, J., Ilschner, S., \& Hart, P. H. (2015). Ultraviolet radiation, vitamin D and multiple sclerosis. In Neurodegenerative disease management (Vol. 5, Issue 5, pp. 413-424). Neurodegener Dis Manag. https://doi.org/10.2217/nmt.15.33

Lucchinetti, C., Brück, W., Parisi, J., Scheithauer, B., Rodriguez, M., \& Lassmann, H. (2000). Heterogeneity of multiple sclerosis lesions: Implications for the pathogenesis of demyelination. Annals of Neurology, 47(6), 707-717. https://doi.org/10.1002/15318249(200006)47:6<707::AID-ANA3>3.0.CO;2-Q

Lumeng, C. N., Bodzin, J. L., \& Saltiel, A. R. (2007). Obesity induces a phenotypic switch in adipose tissue macrophage polarization. Journal of Clinical Investigation, 117(1), 175-184. https://doi.org/10.1172/JCI29881

Magyari, M. (2016). Gender differences in multiple sclerosis epidemiology and treatment response. $J, 63(3)$, B5212. https://ugeskriftet.dk/files/b5473_pulmonary_artery_perfusion_versus_no_pulmonary_perfusi on_during_cardiopulmonary_bypass.pdf

Maldjian, J. A., Laurienti, P. J., Kraft, R. A., \& Burdette, J. H. (2003). An automated method for neuroanatomic and cytoarchitectonic atlas-based interrogation of fMRI data sets. NeuroImage, 19(3), 1233-1239. https://doi.org/10.1016/S1053-8119(03)00169-1

Manca, R., Sharrack, B., Paling, D., Wilkinson, I. D., \& Venneri, A. (2018). Brain connectivity and cognitive processing speed in multiple sclerosis: A systematic review. In Journal of the Neurological Sciences (Vol. 388, pp. 115-127). Elsevier B.V. https://doi.org/10.1016/j.jns.2018.03.003

Martínez-Heras, E., Solana, E., Prados, F., Andorrà, M., Solanes, A., López-Soley, E., Montejo, C., Pulido-Valdeolivas, I., Alba-Arbalat, S., Sola-Valls, N., Sepúlveda, M., Blanco, Y., Saiz, A., Radua, J., \& Llufriu, S. (2020). Characterization of multiple sclerosis lesions with distinct clinical correlates through quantitative diffusion MRI. NeuroImage: Clinical, 28, 102411. https://doi.org/10.1016/j.nicl.2020.102411

Mazerolle, E. L., Wojtowicz, M. A., Omisade, A., \& Fisk, J. D. (2013). Intra-individual variability in information processing speed reflects white matter microstructure in multiple sclerosis. NeuroImage: Clinical, 2(1), 894-902. https://doi.org/10.1016/j.nicl.2013.06.012

McDonald, W. I., Compston, A., Edan, G., Goodkin, D., Hartung, H. P., Lublin, F. D., McFarland, H. F., Paty, D. W., Polman, C. H., Reingold, S. C., Sandberg-Wollheim, M., Sibley, W., Thompson, A., Van Den Noort, S., Weinshenker, B. Y., \& Wolinsky, J. S. (2001). Recommended diagnostic criteria for multiple sclerosis: Guidelines from the International Panel on the Diagnosis of Multiple Sclerosis. Annals of Neurology, 50(1), 121-127. https://doi.org/10.1002/ana.1032

Mesaros, S., Rocca, M. A., Pagani, E., Sormani, M. P., Petrolini, M., Comi, G., \& Filippi, M. (2011). Thalamic damage predicts the evolution of primary-progressive multiple sclerosis at 5 years. American Journal of Neuroradiology, 32(6), 1016-1020. https://doi.org/10.3174/ajnr.A2430 
Minagar, A., Barnett, M. H., Benedict, R. H. B., Pelletier, D., Pirko, I., Sahraian, M. A., Frohman, E., \& Zivadinov, R. (2013). The thalamus and multiple sclerosis: Modern views on pathologic, imaging, and clinical aspects. In Neurology (Vol. 80, Issue 2, pp. 210-219). Neurology. https://doi.org/10.1212/WNL.0b013e31827b910b

Miró-Padilla, A., Bueichekú, E., Ventura-Campos, N., Flores-Compañ, M.-J., Parcet, M. A., \& Ávila, C. (2018). Long-term brain effects of N-back training: an fMRI study. Brain Imaging and Behavior, 1-13. https://doi.org/10.1007/s11682-018-9925-x

Mitolo, M., Venneri, A., Wilkinson, I. D., \& Sharrack, B. (2015). Cognitive rehabilitation in multiple sclerosis: A systematic review. Journal of the Neurological Sciences, 354, 1-9. https://doi.org/10.1016/j.jns.2015.05.004

Mokry, L. E., Ross, S., Ahmad, O. S., Forgetta, V., Smith, G. D., Leong, A., Greenwood, C. M. T., Thanassoulis, G., \& Richards, J. B. (2015). Vitamin D and Risk of Multiple Sclerosis: A Mendelian Randomization Study. PLoS Medicine, 12(8). https://doi.org/10.1371/journal.pmed.1001866

Mollison, D., Sellar, R., Bastin, M., Mollison, D., Chandran, S., Wardlaw, J., \& Connick, P. (2017). The clinico-radiological paradox of cognitive function and MRI burden of white matter lesions in people with multiple sclerosis: A systematic review and meta-analysis. In PLoS ONE (Vol. 12, Issue 5). Public Library of Science. https://doi.org/10.1371/journal.pone.0177727

Morgan, B. P., Gommerman, J. L., \& Ramaglia, V. (2021). An "Outside-In" and "Inside-Out" Consideration of Complement in the Multiple Sclerosis Brain: Lessons From Development and Neurodegenerative Diseases. In Frontiers in Cellular Neuroscience (Vol. 14, p. 442). Frontiers Media S.A. https://doi.org/10.3389/fncel.2020.600656

Munger, K. L., Bentzen, J., Laursen, B., Stenager, E., Koch-Henriksen, N., Sørensen, T. I. A., \& Baker, J. L. (2013). Childhood body mass index and multiple sclerosis risk: A long-term cohort study. Multiple Sclerosis Journal, 19(10), 1323-1329. https://doi.org/10.1177/1352458513483889

Munger, K. L., Hongell, K., Åivo, J., Soilu-Hänninen, M., Surcel, H. M., \& Ascherio, A. (2017). 25-Hydroxyvitamin D deficiency and risk of MS among women in the Finnish Maternity Cohort. Neurology, 89(15), 1578-1583. https://doi.org/10.1212/WNL.0000000000004489

Munger, K. L., Levin, L. I., Hollis, B. W., Howard, N. S., \& Ascherio, A. (2006). Serum 25hydroxyvitamin D levels and risk of multiple sclerosis. Journal of the American Medical Association, 296(23), 2832-2838. https://doi.org/10.1001/jama.296.23.2832

Murphy, R., O’Donoghue, S., Counihan, T., McDonald, C., Calabresi, P. A., Ahmed, M. A. S., Kaplin, A., \& Hallahan, B. (2017). Neuropsychiatric syndromes of multiple sclerosis. In Journal of Neurology, Neurosurgery and Psychiatry (Vol. 88, Issue 8, pp. 697-708). BMJ Publishing Group. https://doi.org/10.1136/jnnp-2016-315367

Muthuraman, M., Fleischer, V., Kolber, P., Luessi, F., Zipp, F., \& Groppa, S. (2016). Structural brain network characteristics can differentiate CIS from early RRMS. Frontiers in Neuroscience, 10(FEB), 14. https://doi.org/10.3389/fnins.2016.00014

Naito, S., Namerow, N., Mickey, M. R., \& Terasaki, P. I. (1972). Multiple Sclerosis: Association with HL-A3. Tissue Antigens, 2(1), 1-4. https://doi.org/10.1111/j.1399-0039.1972.tb00111.x

Nocentini, U., Pasqualetti, P., Bonavita, S., Buccafusca, M., De Caro, M. F., Farina, D., Girlanda, P., Le Pira, F., Lugaresi, A., Quattrone, A., Reggio, A., Salemi, G., Savettieri, G., Tedeschi, 
G., Trojano, M., Valentino, P., \& Caltagirone, C. (2006). Cognitive dysfunction in patients with relapsing-remitting multiple sclerosis. Multiple Sclerosis, 12(1), 77-87.

https://doi.org/10.1191/135248506ms1227oa

Noseworthy, J. H., Lucchinetti, C., Rodriguez, M., \& Weinshenker, B. G. (2000). Multiple

Sclerosis. New England Journal of Medicine, 343(13), 938-952.

https://doi.org/10.1056/NEJM200009283431307

Ntoskou, K., Messinis, L., Nasios, G., Martzoukou, M., Makris, G., Panagiotopoulos, E., \& Papathanasopoulos, P. (2018). Cognitive and Language Deficits in Multiple Sclerosis: Comparison of Relapsing Remitting and Secondary Progressive Subtypes. The Open Neurology Journal, 12(1), 19-30. https://doi.org/10.2174/1874205x01812010019

O'Brien, A. R., Chiaravalloti, N., Goverover, Y., \& DeLuca, J. (2008). Evidenced-Based Cognitive Rehabilitation for Persons With Multiple Sclerosis: A Review of the Literature. In Archives of Physical Medicine and Rehabilitation (Vol. 89, Issue 4, pp. 761-769). Arch Phys Med Rehabil. https://doi.org/10.1016/j.apmr.2007.10.019

Oh, A., Duerden, E. G., \& Pang, E. W. (2014). The role of the insula in speech and language processing. Brain and Language, 135, 96-103. https://doi.org/10.1016/j.bandl.2014.06.003

Ohlmeier, C., Gothe, H., Haas, J., Osowski, U., Weinhold, C., Blauwitz, S., Schmedt, N., Galetzka, W., Berkemeier, F., Tackenberg, B., \& Stangel, M. (2020). Epidemiology, characteristics and treatment of patients with relapsing remitting multiple sclerosis and incidence of high disease activity: Real world evidence based on German claims data. PLOS ONE, 15(5), e0231846. https://doi.org/10.1371/journal.pone.0231846

Olerup, O., \& Hillert, J. (1991). HLA class II-associated genetic susceptibility in multiple sclerosis: A critical evaluation. In Tissue Antigens (Vol. 38, Issue 2, pp. 1-15). Tissue Antigens. https://doi.org/10.1111/j.1399-0039.1991.tb02029.x

Olsson, T., Barcellos, L. F., \& Alfredsson, L. (2016). Interactions between genetic, lifestyle and environmental risk factors for multiple sclerosis. Nature Reviews Neurology, 13(1), 26-36. https://doi.org/10.1038/nrneurol.2016.187

Owen, A. M., McMillan, K. M., Laird, A. R., \& Bullmore, E. (2005). N-back working memory paradigm: A meta-analysis of normative functional neuroimaging studies. Human Brain Mapping, 25(1), 46-59. https://doi.org/10.1002/hbm.20131

Owens, E. M., Denney, D. R., \& Lynch, S. G. (2013). Difficulties in planning among patients with multiple sclerosis: A relative consequence of deficits in information processing speed. Journal of the International Neuropsychological Society, 19(5), 613-620.

https://doi.org/10.1017/S1355617713000155

Parisi, L., Rocca, M. A., Mattioli, F., Copetti, M., Capra, R., Valsasina, P., Stampatori, C., \& Filippi, M. (2014). Changes of brain resting state functional connectivity predict the persistence of cognitive rehabilitation effects in patients with multiple sclerosis. Multiple Sclerosis Journal, 20(6), 686-694. https://doi.org/10.1177/1352458513505692

Parisi, L., Rocca, M. A., Valsasina, P., Panicari, L., Mattioli, F., \& Filippi, M. (2014). Cognitive rehabilitation correlates with the functional connectivity of the anterior cingulate cortex in patients with multiple sclerosis. Brain Imaging and Behavior, 8(3), 387-393. https://doi.org/10.1007/s11682-012-9160-9

Parmenter, B. A., Shucard, J. L., Benedict, R. H. B., \& Shucard, D. W. (2006). Working memory 
deficits in multiple sclerosis: Comparison between the n-back task and the Paced Auditory Serial Addition Test. Journal of the International Neuropsychological Society, 12(5), 677687. https://doi.org/10.1017/S1355617706060826

Parmenter, B. A., Weinstock-Guttman, B., Garg, N., Munschauer, F., \& Benedict, R. H. B. (2007). Screening for cognitive impairment in multiple sclerosis using the Symbol Digit Modalities Test. Multiple Sclerosis, 13(1), 52-57. https://doi.org/10.1177/1352458506070750

Penner, I. K., Kappos, L., Rausch, M., Opwis, K., \& Radü, E. W. (2006). Therapy-induced plasticity of cognitive functions in MS patients: Insights from fMRI. Journal of PhysiologyParis, 99(4-6), 455-462. https://doi.org/10.1016/J.JPHYSPARIS.2006.03.008

Penner, I. K., Opwis, K., \& Kappos, L. (2007). Relation between functional brain imaging, cognitive impairment and cognitive rehabilitation in patients with multiple sclerosis. Journal of Neurology, 254(S2), II53-II57. https://doi.org/10.1007/s00415-007-2013-6

Pérez-Carmona, N., Fernández-Jover, E., \& Sempere, Á. P. (2019). Epidemiology of multiple sclerosis in Spain. Revista de Neurologia, 69(1), 32-38. https://doi.org/10.33588/rn.6901.2018477

Perez-Carmona, N., Gimenez-Martinez, J., Borrego-Honrubia, C., \& Sempere, A. P. (2019). Multiple sclerosis prevalence and incidence in San Vicente del Raspeig, Spain. Multiple Sclerosis and Related Disorders, 33, 78-81. https://doi.org/10.1016/j.msard.2019.05.022

Peterson, J. W., Bö, L., Mörk, S., Chang, A., \& Trapp, B. D. (2001). Transected neurites, apoptotic neurons, and reduced inflammation in cortical multiple sclerosis lesions. Annals of Neurology, 50(3), 389-400. https://doi.org/10.1002/ana.1123

Polman, C. H., Reingold, S. C., Banwell, B., Clanet, M., Cohen, J. A., Filippi, M., Fujihara, K., Havrdova, E., Hutchinson, M., Kappos, L., Lublin, F. D., Montalban, X., O’Connor, P., Sandberg-Wollheim, M., Thompson, A. J., Waubant, E., Weinshenker, B., \& Wolinsky, J. S. (2011). Diagnostic criteria for multiple sclerosis: 2010 Revisions to the McDonald criteria. Annals of Neurology, 69(2), 292-302. https://doi.org/10.1002/ana.22366

Polman, C. H., Reingold, S. C., Edan, G., Filippi, M., Hartung, H.-P., Kappos, L., Lublin, F. D., Metz, L. M., McFarland, H. F., O’Connor, P. W., Sandberg-Wollheim, M., Thompson, A. J., Weinshenker, B. G., \& Wolinsky, J. S. (2005). Diagnostic criteria for multiple sclerosis: 2005 revisions to the "McDonald Criteria." Annals of Neurology, 58(6), 840-846. https://doi.org/10.1002/ana.20703

Popescu, B. F. G., Pirko, I., \& Lucchinetti, C. F. (2013). Pathology of multiple sclerosis: Where do we stand? In CONTINUUM Lifelong Learning in Neurology (Vol. 19, Issue 4, pp. 901-921). American Academy of Neurology. https://doi.org/10.1212/01.CON.0000433291.23091.65

Poser, C. M. (1995). Viking voyages: the origin of multiple sclerosis?: An essay in medical history. Acta Neurologica Scandinavica, 91(161 S), 11-22. https://doi.org/10.1111/j.16000404.1995.tb05852.x

Potagas, C., Giogkaraki, E., Koutsis, G., Mandellos, D., Tsirempolou, E., Sfagos, C., \& Vassilopoulos, D. (2008). Cognitive impairment in different MS subtypes and clinically isolated syndromes. Journal of the Neurological Sciences, 267(1-2), 100-106. https://doi.org/10.1016/J.JNS.2007.10.002

Procaccini, C., Pucino, V., Mantzoros, C. S., \& Matarese, G. (2015). Leptin in autoimmune diseases. In Metabolism: Clinical and Experimental (Vol. 64, Issue 1, pp. 92-104). W.B. 
Saunders. https://doi.org/10.1016/j.metabol.2014.10.014

Prosperini, L., \& Filippo, M. Di. (2019). Beyond clinical changes: Rehabilitation-induced neuroplasticity in MS. Multiple Sclerosis Journal, 25(10), 1348-1362. https://doi.org/10.1177/1352458519846096

Prosperini, L., Piattella, M. C., Giannì, C., \& Pantano, P. (2015). Functional and Structural Brain Plasticity Enhanced by Motor and Cognitive Rehabilitation in Multiple Sclerosis. Neural Plasticity, 2015, 481574. https://doi.org/10.1155/2015/481574

Qian, J., Diez, I., Ortiz-Terán, L., Bonadio, C., Liddell, T., Goñi, J., \& Sepulcre, J. (2018). Positive connectivity predicts the dynamic intrinsic topology of the human brain Network. Frontiers in Systems Neuroscience, 12, 38. https://doi.org/10.3389/fnsys.2018.00038

Ramasamy, D. P., Benedict, R. H. B., Cox, J. L., Fritz, D., Abdelrahman, N., Hussein, S., Minagar, A., Dwyer, M. G., \& Zivadinov, R. (2009). Extent of cerebellum, subcortical and cortical atrophy in patients with MS. A case-control study. Journal of the Neurological Sciences, 282(1-2), 47-54. https://doi.org/10.1016/j.jns.2008.12.034

Rao, S.M., \& Cognitive Function Study Group, N. (1990). A Manual for the Brief Repeatable Battery of Neuropsychology Tests in Multiple Sclerosis National. Multiple Sclerosis Society.

Rao, Stephen M., Leo, G. J., Bernardin, L., \& Unverzagt, F. (1991). Cognitive dysfunction in multiple sclerosis. I. Frequency, patterns, and prediction. Neurology, 41(5), 685-691. https://doi.org/10.1212/WNL.41.5.685

Reddy, H., Narayanan, S., Woolrich, M., Mitsumori, T., Lapierre, Y., Arnold, D. L., \& Matthews, P. M. (2002). Functional brain reorganization for hand movement in patients with multiple sclerosis: Defining distinct effects of injury and disability. Brain, 125(12), 2646-2657. https://doi.org/10.1093/brain/awf283

Redick, T. S., \& Lindsey, D. R. B. (2013). Complex span and n-back measures of working memory: A meta-analysis. Psychonomic Bulletin and Review, 20(6), 1102-1113. https://doi.org/10.3758/s13423-013-0453-9

Reicker, L. I., Tombaugh, T. N., Walker, L., \& Freedman, M. S. (2007). Reaction time: An alternative method for assessing the effects of multiple sclerosis on information processing speed. Archives of Clinical Neuropsychology, 22(5), 655-664. https://doi.org/10.1016/j.acn.2007.04.008

Rhead, B., Bäärnhielm, M., Gianfrancesco, M., Mok, A., Shao, X., Quach, H., Shen, L., Schaefer, C., Link, J., Gyllenberg, A., Hedström, A. K., Olsson, T., Hillert, J., Kockum, I., Glymour, M. M., Alfredsson, L., \& Barcellos, L. F. (2016). Mendelian randomization shows a causal effect of low Vitamin D on multiple sclerosis risk. Neurology: Genetics, 2(5). https://doi.org/10.1212/NXG.0000000000000097

Ricigliano, V. A. G., Tonietto, M., Palladino, R., Poirion, E., De Luca, A., Branzoli, F., Bera, G., Maillart, E., Stankoff, B., \& Bodini, B. (2021). Thalamic energy dysfunction is associated with thalamo-cortical tract damage in multiple sclerosis: A diffusion spectroscopy study. Multiple Sclerosis Journal, 27(4), 528-538. https://doi.org/10.1177/1352458520921362

Rimkus, C., Junqueira, T., Lyra, K., Jackowski, M. P., Machado, M. A. R., Miotto, E. C., Callegaro, D., Otaduy, M. C., \& Leite, C. (2011). Corpus Callosum Microstructural Changes Correlate with Cognitive Dysfunction in Early Stages of Relapsing-Remitting Multiple Sclerosis: Axial and Radial Diffusivities Approach. Multiple Sclerosis International, 2011, 1-7. 
https://doi.org/10.1155/2011/304875

Rinaldi, F., Calabrese, M., Grossi, P., Puthenparampil, M., Perini, P., \& Gallo, P. (2010). Cortical lesions and cognitive impairment in multiple sclerosis. Neurological Sciences, 31(SUPPL. 2). https://doi.org/10.1007/s10072-010-0368-4

Rocca, M. A., Absinta, M., Moiola, L., Ghezzi, A., Colombo, B., Martinelli, V., Comi, G., \& Filippi, M. (2010). Functional and structural connectivity of the motor network in pediatric and adult-onset relapsing-remitting multiple sclerosis. Radiology, 254(2), 541-550. https://doi.org/10.1148/radiol.09090463

Rocca, M. A., Amato, M. P., De Stefano, N., Enzinger, C., Geurts, J. J., Penner, I. K., Rovira, A., Sumowski, J. F., Valsasina, P., \& Filippi, M. (2015). Clinical and imaging assessment of cognitive dysfunction in multiple sclerosis. The Lancet Neurology, 14(3), 302-317. https://doi.org/10.1016/S1474-4422(14)70250-9

Rocca, M. A., Hidalgo de La Cruz, M., Valsasina, P., Mesaros, S., Martinovic, V., Ivanovic, J., Drulovic, J., \& Filippi, M. (2020). Two-year dynamic functional network connectivity in clinically isolated syndrome. Multiple Sclerosis Journal, 26(6), 645-658. https://doi.org/10.1177/1352458519837704

Rocca, M. A., Mesaros, S., Pagani, E., Sormani, M. P., Comi, G., \& Filippi, M. (2010). Thalamic damage and long-term progression of disability in multiple sclerosis. Radiology, 257(2), 463469. https://doi.org/10.1148/radiol.10100326

Rocca, M. A., Valsasina, P., Absinta, M., Riccitelli, G., Rodegher, M. E., Misci, P., Rossi, P., Falini, A., Comi, G., \& Filippi, M. (2010). Default-mode network dysfunction and cognitive impairment in progressive MS. Neurology, 74(16), 1252-1259. https://doi.org/10.1212/WNL.0b013e3181d9ed91

Rojas, J. I., Murphy, G., Sanchez, F., Patrucco, L., Fernandez, M. C., Miguez, J., Funes, J., Golimstok, A., \& Cristiano, E. (2018). Thalamus volume change and cognitive impairment in early relapsing-remitting multiple sclerosis patients. Neuroradiology Journal, 31(4), 350-355. https://doi.org/10.1177/1971400918781977

Roosendaal, S. D., Schoonheim, M. M., Hulst, H. E., Sanz-Arigita, E. J., Smith, S. M., Geurts, J. J. G., \& Barkhof, F. (2010). Resting state networks change in clinically isolated syndrome. Brain, 133(6), 1612-1621. https://doi.org/10.1093/brain/awq058

Rosti-Otajärvi, E. M., \& Hämäläinen, P. I. (2014). Neuropsychological rehabilitation for multiple sclerosis. In Cochrane Database of Systematic Reviews (Vol. 2014, Issue 2). John Wiley and Sons Ltd. https://doi.org/10.1002/14651858.CD009131.pub3

Roth, A. K., Denney, D. R., \& Lynch, S. G. (2015). Information processing speed and attention in multiple sclerosis: Reconsidering the Attention Network Test (ANT). Journal of Clinical and Experimental Neuropsychology, 37(5), 518-529. https://doi.org/10.1080/13803395.2015.1037252

Rotstein, D. L., Marrie, R. A., Maxwell, C., Gandhi, S., Schultz, S. E., Fung, K., \& Tu, K. (2019). MS risk in immigrants in the McDonald era: A population-based study in Ontario, Canada. Neurology, 93(24), E2203-E2215. https://doi.org/10.1212/WNL.0000000000008611

Rovaris, M., Filippi, M., Falautano, M., Minicucci, L., Rocca, M. A., Martinelli, V., \& Comi, G. (1998). Relation between MR abnormalities and patterns of cognitive impairment in multiple sclerosis. Neurology, 50(6), 1601-1608. https://doi.org/10.1212/WNL.50.6.1601 
Rovaris, M., Iannucci, G., Falautano, M., Possa, F., Martinelli, V., Comi, G., \& Filippi, M. (2002). Cognitive dysfunction in patients with mildly disabling relapsing-remitting multiple sclerosis: An exploratory study with diffusion tensor MR imaging. Journal of the Neurological Sciences, 195(2), 103-109. https://doi.org/10.1016/S0022-510X(01)00690-6

Rubinov, M., \& Sporns, O. (2010). Complex network measures of brain connectivity: Uses and interpretations. NeuroImage, 52(3), 1059-1069. https://doi.org/10.1016/j.neuroimage.2009.10.003

Ruchkin, D. S., Grafman, J., Krauss, G. L., Johnson, R., Canoune, H., \& Ritter, W. (1994). Eventrelated brain potential evidence for a verbal working memory deficit in multiple sclerosis. Brain, 117(2), 289-305. https://doi.org/10.1093/brain/117.2.289

Sadovnick, A. D., Armstrong, H., Rice, G. P. A., Bulman, D., Hashimoto, L., Party, D. W., Hashimoto, S. A., Warren, S., Hader, W., Murrary, T. J., Seland, T. P., Metz, L., Bell, R., Duquette, P., Gray, T., Nelson, R., Weinshenkar, B., Brunt, D., \& Ebers, G. C. (1993). A population-based study of multiple sclerosis in twins: Update. Annals of Neurology, 33(3), 281-285. https://doi.org/10.1002/ana.410330309

Salminen, T., Strobach, T., \& Schubert, T. (2012). On the impacts of working memory training on executive functioning. Frontiers in Human Neuroscience, 6, 166. https://doi.org/10.3389/fnhum.2012.00166

Salzer, J., Hallmans, G., Nyström, M., Stenlund, H., Wadell, G., \& Sundström, P. (2012). VitaminDas a protective factor in multiple sclerosis. Neurology, 79(21), 2140-2145. https://doi.org/10.1212/WNL.0b013e3182752ea8

Sánchez, M. P., Nieto, A., Barroso, J., Martín, V., \& Hernández, M. A. (2008). Brain atrophy as a marker of cognitive impairment in mildly disabling relapsing-remitting multiple sclerosis. European Journal of Neurology, 15(10), 1091-1099. https://doi.org/10.1111/j.14681331.2008.02259.x

Sanfilipo, M. P., Benedict, R. H. ., Zivadinov, R., \& Bakshi, R. (2004). Correction for intracranial volume in analysis of whole brain atrophy in multiple sclerosis: the proportion vs. residual method. NeuroImage, 22(4), 1732-1743. https://doi.org/10.1016/J.NEUROIMAGE.2004.03.037

Sanfilipo, M. P., Benedict, R. H. B., Weinstock-Guttman, B., \& Bakshi, R. (2006). Gray and white matter brain atrophy and neuropsychological impairment in multiple sclerosis. Neurology, 66(5), 685-692. https://doi.org/10.1212/01.wnl.0000201238.93586.d9

Sastre-Garriga, J., Alonso, J., Renom, M., Arévalo, M. J., González, I., Galán, I., Montalban, X., \& Rovira, A. (2010). A functional magnetic resonance proof of concept pilot trial of cognitive rehabilitation in multiple sclerosis. Multiple Sclerosis Journal, 17(4), 457-467. https://doi.org/10.1177/1352458510389219

Sastre-Garriga, J., Ingle, G. T., Chard, D. T., Cercignani, M., Ramió-Torrentà, L., Miller, D. H., \& Thompson, A. J. (2005). Grey and white matter volume changes in early primary progressive multiple sclerosis: a longitudinal study. Brain, 128(6), 1454-1460. https://doi.org/10.1093/brain/awh498

Sastre-Garriga, J., Pareto, D., \& Rovira, A. (2017). Brain Atrophy in Multiple Sclerosis: Clinical Relevance and Technical Aspects. Neuroimaging Clinics of North America, 27(2), 289-300. https://doi.org/10.1016/j.nic.2017.01.002 
Sbardella, E., Tona, F., Petsas, N., Upadhyay, N., Piattella, M. C., Filippini, N., Prosperini, L., Pozzilli, C., \& Pantano, P. (2015). Functional connectivity changes and their relationship with clinical disability and white matter integrity in patients with relapsing-remitting multiple sclerosis. Multiple Sclerosis, 21(13), 1681-1692. https://doi.org/10.1177/1352458514568826

Schmidt, P. (2017). Bayesian inference for structured additive regression models for large-scale problems with applications to medical imaging.

Schmidt, P., Gaser, C., Arsic, M., Buck, D., Förschler, A., Berthele, A., Hoshi, M., Ilg, R., Schmid, V. J., Zimmer, C., Hemmer, B., \& Mühlau, M. (2012). An automated tool for detection of FLAIR-hyperintense white-matter lesions in Multiple Sclerosis. NeuroImage, 59(4), 37743783. https://doi.org/10.1016/J.NEUROIMAGE.2011.11.032

Schneiders, J. A., Opitz, B., Tang, H., Deng, Y., Xie, C., Li, H., \& Mecklinger, A. (2012). The impact of auditory working memory training on the fronto-parietal working memory network. Frontiers in Human Neuroscience, 6, 173. https://doi.org/10.3389/fnhum.2012.00173

Schoonheim, M. M., Hulst, H. E., Brandt, R. B., Strik, M., Wink, A. M., Uitdehaag, B. M. J., Barkhof, F., \& Geurts, J. J. G. (2015). Thalamus structure and function determine severity of cognitive impairment in multiple sclerosis. Neurology, 84(8), 776-783. https://doi.org/10.1212/WNL.0000000000001285

Schoonheim, M. M., Meijer, K. A., \& Geurts, J. J. G. (2015). Network collapse and cognitive impairment in multiple sclerosis. Frontiers in Neurology, 6(MAR). https://doi.org/10.3389/fneur.2015.00082

Schreiber, K., Sørensen, P. S., Koch-Henriksen, N., Wagner, A., Blinkenberg, M., Svarer, C., \& Petersen, H. C. (2001). Correlations of brain MRI parameters to disability in multiple sclerosis. Acta Neurologica Scandinavica, 104(1), 24-30. https://doi.org/10.1034/j.16000404.2001.00041.x

Sen, M. K., Almuslehi, M. S. M., Shortland, P. J., Coorssen, J. R., \& Mahns, D. A. (2020). Revisiting the Pathoetiology of Multiple Sclerosis: Has the Tail Been Wagging the Mouse? Frontiers in Immunology, 11, 572186. https://doi.org/10.3389/fimmu.2020.572186

Sepulcre, J., Liu, H., Talukdar, T., Martincorena, I., Yeo, B. T., \& Buckner, R. L. (2010). The organization of local and distant functional connectivity in the human brain. PLoS Computational Biology, 6(6), 1-15. https://doi.org/10.1371/journal.pcbi.1000808

Sepulcre, J., Masdeu, J. C., Pastor, M. A., Goñi, J., Barbosa, C., Bejarano, B., \& Villoslada, P. (2009). Brain pathways of verbal working memory. A lesion-function correlation study. NeuroImage, 47(2), 773-778. https://doi.org/10.1016/j.neuroimage.2009.04.054

Sepulcre, J., Sabuncu, M. R., \& Johnson, K. A. (2012). Network assemblies in the functional brain. Current Opinion in Neurology, 25(4), 384-391. https://doi.org/10.1097/WCO.0b013e328355a8e8

Sepulcre, J., Sabuncu, M. R., Yeo, T. B., Liu, H., \& Johnson, K. A. (2012). Stepwise Connectivity of the Modal Cortex Reveals the Multimodal Organization of the Human Brain. The Journal of Neuroscience, 32(31), 10649-10661. https://doi.org/10.1523/JNEUROSCI.0759-12.2012

Sepulcre, J., Sastre-Garriga, J., Cercignani, M., Ingle, G. T., Miller, D. H., \& Thompson, A. J. (2006). Regional gray matter atrophy in early primary progressive multiple sclerosis: A voxelbased morphometry study. Archives of Neurology, 63(8), 1175-1180.

https://doi.org/10.1001/archneur.63.8.1175 
Sepulcre, J., Vannotti, S., Hernández, R., Sandoval, G., Cáceres, F., Garcea, O., \& Villoslada, P. (2006). Cognitive impairment in patients with multiple sclerosis using the Brief Repeatable Battery-Neuropsychology test. Multiple Sclerosis, 12(2), 187-195. https://doi.org/10.1191/1352458506ms1258oa

Shinoda, K., Matsushita, T., Nakamura, Y., Masaki, K., Sakai, S., Nomiyama, H., Togao, O., Hiwatashi, A., Niino, M., Isobe, N., \& Kira, J. ichi. (2020). Contribution of cortical lesions to cognitive impairment in Japanese patients with multiple sclerosis. Scientific Reports, 10(1). https://doi.org/10.1038/s41598-020-61012-3

Sicotte, N. L., Kern, K. C., Giesser, B. S., Arshanapalli, A., Schultz, A., Montag, M., Wang, H., \& Bookheimer, S. Y. (2008). Regional hippocampal atrophy in multiple sclerosis. Brain, 131(4), 1134-1141. https://doi.org/10.1093/brain/awn030

Simpson, S., Wang, W., Otahal, P., Blizzard, L., Van Der Mei, I. A. F., \& Taylor, B. V. (2019). Latitude continues to be significantly associated with the prevalence of multiple sclerosis: An updated meta-analysis. Journal of Neurology, Neurosurgery and Psychiatry, 90(11), 11931200. https://doi.org/10.1136/jnnp-2018-320189

Skegg, D. C. G., Corwin, P. A., Craven, R. S., Malloch, J. A., \& Pollock, M. (1987). Occurrence of multiple sclerosis in the north and south of New Zealand. Journal of Neurology Neurosurgery and Psychiatry, 50(2), 134-139. https://doi.org/10.1136/jnnp.50.2.134

Soares, J. M., Conde, R., Magalhães, R., Marques, P., Magalhães, R., Gomes, L., Gonçalves, Ó. F., Arantes, M., \& Sampaio, A. (2021). Alterations in functional connectivity are associated with white matter lesions and information processing efficiency in multiple sclerosis. Brain Imaging and Behavior, 15(1), 375-388. https://doi.org/10.1007/s11682-020-00264-z

Sociedad Española de Neurología. (2016). 8 de diciembre: Día Nacional de la Esclerosis Múltiple. https://www.sen.es/saladeprensa/pdf/Link204.pdf

Spain, R. I., Maltenfort, M., Sergott, R. C., \& Leist, T. P. (2009). Thickness of retinal nerve fiber layer correlates with disease duration in parallel with corticospinal tract dysfunction in untreated multiple sclerosis. Journal of Rehabilitation Research and Development, 46(5), 633-642. https://doi.org/10.1682/JRRD.2008.11.0156

Squire, L. R., \& Zola-Morgan, S. (1991). The medial temporal lobe memory system. In Science (Vol. 253, Issue 5026, pp. 1380-1386). Science. https://doi.org/10.1126/science.1896849

Swirsky-Sacchetti, T., Mitchell, D. R., Seward, J., Gonzales, C., Lublin, F., Knobler, R., \& Field, H. L. (1992). Neuropsychological and structural brain lesions in multiple sclerosis: A regional analysis. Neurology, 42(7), 1291-1295. https://doi.org/10.1212/wnl.42.7.1291

Tahedl, M., Levine, S. M., Greenlee, M. W., Weissert, R., \& Schwarzbach, J. V. (2018). Functional Connectivity in Multiple Sclerosis: Recent Findings and Future Directions. Frontiers in Neurology, 9, 828. https://doi.org/10.3389/fneur.2018.00828

Tewarie, P., Steenwijk, M. D., Brookes, M. J., Uitdehaag, B. M. J., Geurts, J. J. G., Stam, C. J., \& Schoonheim, M. M. (2018). Explaining the heterogeneity of functional connectivity findings in multiple sclerosis: An empirically informed modeling study. Human Brain Mapping, 39(6), 2541-2548. https://doi.org/10.1002/hbm.24020

Thompson, A. J., Banwell, B. L., Barkhof, F., Carroll, W. M., Coetzee, T., Comi, G., Correale, J., Fazekas, F., Filippi, M., Freedman, M. S., Fujihara, K., Galetta, S. L., Hartung, H. P., Kappos, L., Lublin, F. D., Marrie, R. A., Miller, A. E., Miller, D. H., Montalban, X., ... Cohen, J. A. 
(2018). Diagnosis of multiple sclerosis: 2017 revisions of the McDonald criteria. In The Lancet Neurology (Vol. 17, Issue 2, pp. 162-173). Lancet Publishing Group.

https://doi.org/10.1016/S1474-4422(17)30470-2

Thompson, T. W., Waskom, M. L., \& Gabrieli, J. D. E. (2016). Intensive working memory training produces functional changes in large-scale frontoparietal networks. Journal of Cognitive Neuroscience, 28(4), 575-588. https://doi.org/10.1162/jocn_a_00916

Thornton, A. E., Raz, N., \& Tucker, K. A. (2002). Memory in multiple sclerosis: Contextual encoding deficits. Journal of the International Neuropsychological Society, 8(3), 395-409. https://doi.org/10.1017/S1355617702813200

Tiberio, M., Chard, D. T., Altmann, D. R., Davies, G., Griffin, C. M., Rashid, W., Sastre-Garriga, J., Thompson, A. J., \& Miller, D. H. (2005). Gray and white matter volume changes in early RRMS: A 2-year longitudinal study. Neurology, 64(6), 1001-1007. https://doi.org/10.1212/01.WNL.0000154526.22878.30

Titus, H. E., Chen, Y., Podojil, J. R., Robinson, A. P., Balabanov, R., Popko, B., \& Miller, S. D. (2020). Pre-clinical and Clinical Implications of "Inside-Out" vs. "Outside-In" Paradigms in Multiple Sclerosis Etiopathogenesis. In Frontiers in Cellular Neuroscience (Vol. 14, p. 357). Frontiers Media S.A. https://doi.org/10.3389/fncel.2020.599717

Tomassini, V., Matthews, P. M., Thompson, A. J., Fuglø, D., Geurts, J. J., Johansen-Berg, H., Jones, D. K., Rocca, M. A., Wise, R. G., Barkhof, F., \& Palace, J. (2012). Neuroplasticity and functional recovery in multiple sclerosis. Nature Reviews. Neurology, 8(11), 635-646. https://doi.org/10.1038/nrneurol.2012.179

Trenova, A. G., Slavov, G. S., Manova, M. G., Aksentieva, J. B., Miteva, L. D., \& Stanilova, S. A. (2016). Cognitive Impairment in Multiple Sclerosis. Folia Medica, 58(3), 157-163. https://doi.org/10.1515/folmed-2016-0029

Tzourio-Mazoyer, N., Landeau, B., Papathanassiou, D., Crivello, F., Etard, O., Delcroix, N., Mazoyer, B., \& Joliot, M. (2002). Automated anatomical labeling of activations in SPM using a macroscopic anatomical parcellation of the MNI MRI single-subject brain. NeuroImage, 15(1), 273-289. https://doi.org/10.1006/nimg.2001.0978

Uher, T., Blahova-Dusankova, J., Horakova, D., Bergsland, N., Tyblova, M., Benedict, R. H. B., Kalincik, T., Ramasamy, D. P., Seidl, Z., Hagermeier, J., Vaneckova, M., Krasensky, J., Havrdova, E., \& Zivadinov, R. (2014). Longitudinal MRI and neuropsychological assessment of patients with clinically isolated syndrome. Journal of Neurology, 261(9), 1735-1744. https://doi.org/10.1007/s00415-014-7413-9

Vacchi, L., Rocca, M. A., Meani, A., Rodegher, M., Martinelli, V., Comi, G., Falini, A., \& Filippi, M. (2017). Working memory network dysfunction in relapse-onset multiple sclerosis phenotypes: A clinical-imaging evaluation. Multiple Sclerosis, 23(4), 577-587. https://doi.org/10.1177/1352458516656809

Valsasina, P., Benedetti, B., Rovaris, M., Sormani, M. P., Comi, G., \& Filippi, M. (2005). Evidence for progressive gray matter loss in patients with relapsing-remitting MS. Neurology, 65(7), 1126-1128. https://doi.org/10.1212/01.wnl.0000178982.53965.70

Van Schependom, J., D’Hooghe, M. B., Cleynhens, K., D’Hooge, M., Haelewyck, M. C., De Keyser, J., \& Nagels, G. (2015). Reduced information processing speed as primum movens for cognitive decline in MS. Multiple Sclerosis Journal, 21(1), 83-91. https://doi.org/10.1177/1352458514537012 
Vaneckova, M., Kalincik, T., Krasensky, J., Horakova, D., Havrdova, E., Hrebikova, T., \& Seidl, Z. (2012). Corpus callosum atrophy - A simple predictor of multiple sclerosis progression: A longitudinal 9-year study. European Neurology, 68(1), 23-27. https://doi.org/10.1159/000337683

Venables, W. N., \& Ripley, B. D. (2002). Modern Applied Statistics with S (Springer (ed.); 4th Ed).

Vercellino, M., Plano, F., Votta, B., Mutani, R., Giordana, M. T., \& Cavalla, P. (2005). Grey matter pathology in multiple sclerosis. Journal of Neuropathology and Experimental Neurology, 64(12), 1101-1107. https://doi.org/10.1097/01.jnen.0000190067.20935.42

Vrenken, H., \& Geurts, J. J. G. (2007). Gray and normal-appearing white matter in multiple sclerosis: An MRI perspective. In Expert Review of Neurotherapeutics (Vol. 7, Issue 3, pp. 271-279). Expert Rev Neurother. https://doi.org/10.1586/14737175.7.3.271

Wager, T. D., \& Smith, E. E. (2003). Neuroimaging studies of working memory: A meta-analysis. In Cognitive, Affective and Behavioral Neuroscience (Vol. 3, Issue 4, pp. 255-274). Psychonomic Society Inc. https://doi.org/10.3758/CABN.3.4.255

Walton, C., King, R., Rechtman, L., Kaye, W., Leray, E., Marrie, R. A., Robertson, N., La Rocca, N., Uitdehaag, B., van der Mei, I., Wallin, M., Helme, A., Angood Napier, C., Rijke, N., \& Baneke, P. (2020). Rising prevalence of multiple sclerosis worldwide: Insights from the Atlas of MS, third edition. Multiple Sclerosis Journal, 26(14), 1816-1821. https://doi.org/10.1177/1352458520970841

Wang, H., He, W., Wu, J., Zhang, J., Jin, Z., \& Li, L. (2019). A coordinate-based meta-analysis of the n-back working memory paradigm using activation likelihood estimation. Brain and Cognition, 132, 1-12. https://doi.org/10.1016/j.bandc.2019.01.002

Watanabe, M., Nakamura, Y., Sato, S., Niino, M., Fukaura, H., Tanaka, M., Ochi, H., Kanda, T., Takeshita, Y., Yokota, T., Nishida, Y., Matsui, M., Nagayama, S., Kusunoki, S., Miyamoto, K., Mizuno, M., Kawachi, I., Saji, E., Ohashi, T., ... Kira, J. ichi. (2021). HLA genotypeclinical phenotype correlations in multiple sclerosis and neuromyelitis optica spectrum disorders based on Japan MS/NMOSD Biobank data. Scientific Reports, 11(1). https://doi.org/10.1038/s41598-020-79833-7

Wegner, C., Esiri, M. M., Chance, S. A., Palace, J., \& Matthews, P. M. (2006). Neocortical neuronal, synaptic, and glial loss in multiple sclerosis. Neurology, 67(6), 960-967. https://doi.org/10.1212/01.wnl.0000237551.26858.39

Weinshenker, B. G., Bass, B., Rice, G. P. A., Noseworthy, J., Carriere, W., Baskerville, J., \& Ebers, G. C. (1989). The natural history of multiple sclerosis: A geographically based study: I. Clinical course and disability. Brain, 112(1), 133-146. https://doi.org/10.1093/brain/112.1.133

Welton, T., Kent, D., Constantinescu, C. S., Auer, D. P., \& Dineen, R. A. (2015). Functionally Relevant White Matter Degradation in Multiple Sclerosis: A Tract-based Spatial MetaAnalysis. Radiology, 275(1), 89-96. https://doi.org/10.1148/radiol.14140925

Whitfield-Gabrieli, S., \& Nieto-Castanon, A. (2012). Conn: A Functional Connectivity Toolbox for Correlated and Anticorrelated Brain Networks. Brain Connectivity, 2(3), 125-141. https://doi.org/10.1089/brain.2012.0073

Willer, C. J., Dyment, D. A., Risch, N. J., Sadovnick, A. D., Ebers, G. C., Paty, D. W., Hashimoto, S. A., Devonshire, V., Hooge, J., Oger, J., Metz, L., Warren, S., Hader, W., Nelson, R., Freedman, M., Brunet, D., Paulseth, J., Rice, G., O’Connor, P., ... Stefanelli, M. (2003). Twin 
concordance and sibling recurrence rates in multiple sclerosis. Proceedings of the National Academy of Sciences of the United States of America, 100(22), 12877-12882.

https://doi.org/10.1073/pnas.1932604100

Winkelmann, A., Engel, C., Apel, A., \& Zettl, U. K. (2007). Cognitive impairment in multiple sclerosis. Journal of Neurology, 254(2 SUPPL.). https://doi.org/10.1007/s00415-007-2010-9

Wood, S. N. (2004). Stable and efficient multiple smoothing parameter estimation for generalized additive models. Journal of the American Statistical Association, 99(467), 673-686. https://doi.org/10.1198/016214504000000980

Wortsman, J., Matsuoka, L. Y., Chen, T. C., Lu, Z., \& Holick, M. F. (2000). Decreased bioavailability of vitamin D in obesity. American Journal of Clinical Nutrition, 72(3), 690693. https://doi.org/10.1093/ajcn/72.3.690

Wylezinska, M., Cifelli, A., Jezzard, P., Palace, J., Alecci, M., \& Matthews, P. M. (2003). Thalamic neurodegeneration in relapsing-remitting multiple sclerosis. Neurology, 60(12), 1949-1954. https://doi.org/10.1212/01.WNL.0000069464.22267.95

Yaldizli, Ö., Atefy, R., Gass, A., Sturm, D., Glassl, S., Tettenborn, B., \& Putzki, N. (2010). Corpus callosum index and long-term disability in multiple sclerosis patients. Journal of Neurology, 257(8), 1256-1264. https://doi.org/10.1007/s00415-010-5503-x

Yamout, B., \& Al Khawajah, M. (2017). Radiologically isolated syndrome and multiple sclerosis. In Multiple Sclerosis and Related Disorders (Vol. 17, pp. 234-237). Elsevier B.V. https://doi.org/10.1016/j.msard.2017.08.016

Yeo, B. T., Krienen, F. M., Sepulcre, J., Sabuncu, M. R., Lashkari, D., Hollinshead, M., Roffman, J. L., Smoller, J. W., Zöllei, L., Polimeni, J. R., Fisch, B., Liu, H., \& Buckner, R. L. (2011). The organization of the human cerebral cortex estimated by intrinsic functional connectivity. Journal of Neurophysiology, 106(3), 1125-1165. https://doi.org/10.1152/jn.00338.2011

Yu, H. J., Christodoulou, C., Bhise, V., Greenblatt, D., Patel, Y., Serafin, D., Maletic-Savatic, M., Krupp, L. B., \& Wagshul, M. E. (2012). Multiple white matter tract abnormalities underlie cognitive impairment in RRMS. NeuroImage, 59(4), 3713-3722. https://doi.org/10.1016/j.neuroimage.2011.10.053

Zakzanis, K. K. (2000). Distinct neurocognitive profiles in multiple sclerosis subtypes. Archives of Clinical Neuropsychology: The Official Journal of the National Academy of Neuropsychologists, 15(2), 115-136.

Zhong, J., Chen, D. Q., Nantes, J. C., Holmes, S. A., Hodaie, M., \& Koski, L. (2017). Combined structural and functional patterns discriminating upper limb motor disability in multiple sclerosis using multivariate approaches. Brain Imaging and Behavior, 11(3), 754-768. https://doi.org/10.1007/s11682-016-9551-4

Zhong, J., Nantes, J. C., Holmes, S. A., Gallant, S., Narayanan, S., \& Koski, L. (2016). Abnormal functional connectivity and cortical integrity influence dominant hand motor disability in multiple sclerosis: a multimodal analysis. Human Brain Mapping, 37(12), 4262-4275. https://doi.org/10.1002/hbm.23307

Zhou, F., Gong, H., Chen, Q., Wang, B., Peng, Y., Zhuang, Y., \& Zee, C. S. (2016). Intrinsic functional plasticity of the thalamocortical system in minimally disabled patients with relapsing-remitting multiple sclerosis. Frontiers in Human Neuroscience, 10(JAN2016). https://doi.org/10.3389/fnhum.2016.00002 
Zou, Q., Ross, T. J., Gu, H., Geng, X., Zuo, X.-N., Hong, L. E., Gao, J.-H., Stein, E. A., Zang, Y.F., \& Yang, Y. (2013). Intrinsic resting-state activity predicts working memory brain activation and behavioral performance. Human Brain Mapping, 34(12), 3204-3215. https://doi.org/10.1002/hbm.22136 


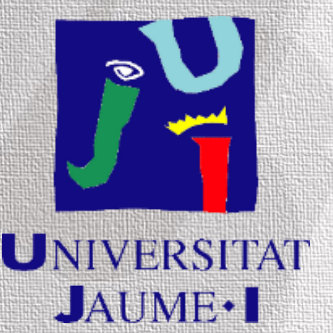

